\title{
Erzeugung und Untersuchung von schnellen Mikrotropfen für Reinigungsanwendungen
}

\author{
DiSSERTATION \\ zur Erlangung des mathematisch-naturwissenschaftlichen Doktorgrades \\ "Doctor rerum naturalium" \\ der Georg-August-Universität Göttingen \\ im Promotionsprogramm ProPhys \\ der Georg-August University School of Science (GAUSS) \\ vorgelegt von \\ PHILIPP ERHARD FROMMHOLD \\ aus \\ Wismar
}

Göttingen 2015 
Betreuungsausschuss

Prof. Dr. Christoph F. Schmidt,

Drittes Physikalisches Institut, Universität Göttingen

Dr. Robert Mettin,

Drittes Physikalisches Institut, Universität Göttingen

$\underline{\text { Mitglieder der Prüfungskommission }}$

Referent: $\quad$ Prof. Dr. Christoph F. Schmidt,

Drittes Physikalisches Institut, Universität Göttingen

Korreferent: Prof. Dr. Stefan Luther,

Biomedical Physics Group, MPIDS Göttingen

Drittreferent: Prof. Dr. Günter Brenn,

Institut für Strömungslehre und Wärmeübertragung,

Technische Universität Graz

Weitere Mitglieder der Prüfungskommission:

Prof. Dr. Jörg Enderlein,

Drittes Physikalisches Institut, Universität Göttingen

Prof. Dr. Wolfram Kollatschny,

Institut für Astrophysik, Universität Göttingen

Prof. Dr. Martin Rein,

Institut für Aerodynamik und Strömungstechnik, DLR Göttingen

Prof. Dr. Florentin Wörgötter,

Drittes Physikalisches Institut, Universität Göttingen

Tag der mündlichen Prüfung: 20.05.2015 




\section{Inhaltsverzeichnis}

\begin{tabular}{ll}
\hline Einleitung & 1
\end{tabular}

\begin{tabular}{|l|l|}
\hline 1. Erzeugung monodisperser Hochgeschwindigkeits-Sprays & 7
\end{tabular}

1.1. Motivation . . . . . . . . . . . . 7

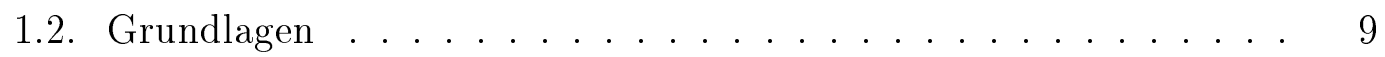

1.2.1. Lineare Stabilitätsanalyse . . . . . . . . . . . . 9

1.2.2. Akustisch gesteuerter Zerfall eines Flüssigkeitsstrahls . . . 10

1.3. Experimenteller Aufbau . . . . . . . . . . . . . . . . 13

1.3.1. Variante A: Edelstahlscheibe . . . . . . . . . . . . 16

1.3.2. Variante B: Siliziummembran ........... . 18

1.4. Auswertung . . . . . . . . . . . . . . . . . . . . . . . . . 19

1.4.1. Strahlparameter für verschiedene Flüssigkeiten . . . . . . . 19

1.4.2. Begrenzung der Tropfengeschwindigkeit . . . . . . . . . . 22

1.4.3. Anpassung der Tropfengröße . . . . . . . . . . . . . . . . 26

1.4.4. Zerfallslänge des Flüssigkeitsstrahls . . . . . . . . . . . 28

1.4.5. Verteilung der Tropfengröße und -geschwindigkeit . . . . . 30

1.4.6. Vergleich unterschiedlicher Düsendurchmesser . . . . . . . 35

1.5. Zusammenfassung . . . . . . . . . . . . . . . 37

2. Erzeugung und Untersuchung des Aufpralls einzelner Mikrotropfen 41

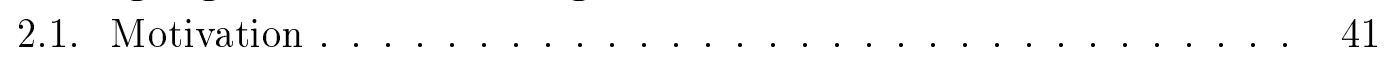

2.2. Experimenteller Aufbau . . . . . . . . . . . . . . . 43

2.2.1. Aufladung und Ablenkung von Einzeltropfen . . . . . . . . . . 44

2.2.2. Aufnahmetechnik . . . . . . . . . . . . . 47

2.3. Auswertung zur Erzeugung einzelner Mikrotropfen . . . . . . . . . 51

2.3.1. Prozess der Tropfenaufladung . . . . . . . . . . . . . 51

2.3.2. Bewegungsgleichungen im elektrischen Feld . . . . . . . . 58

2.3.3. Limitierung der Methode . . . . . . . . . . . . . 74

2.4. Auswertung zum Aufprall einzelner Mikrotropfen . . . . . . . . . . 83

2.4.1. Ansicht von der Seite und von unten . . . . . . . . . . 83 
2.4.2. Ausbreitungsdynamik . . . . . . . . . . . . 87

2.4.3. Maximaler Ausbreitungsdurchmesser . . . . . . . . . . 90

2.4.4. Tropfenprofil . . . . . . . . . . . . . . . . 91

2.5. Zusammenfassung . . . . . . . . . . . . . . . 95

3. Messung der substratnahen Strömungsgeschwindigkeit beim konti-

\begin{tabular}{|lll}
\hline nuierlichen Auftreffen von Tropfen auf ein rotierendes Substrat & 101
\end{tabular}

3.1. Motivation . . . . . . . . . . . . . . . 101

3.2. Experimenteller Aufbau . . . . . . . . . . . . . . 103

3.3. Auswertung . . . . . . . . . . . . . . . . . . 111

3.3.1. Flüssigkeitsfilm auf einem rotierenden Substrat . . . . . . 111

3.3.2. Entwicklung des Einschlagskraters . . . . . . . . . . 113

3.3.3. Messung der substratnahen Strömungsgeschwindigkeit . . 115

3.4. Zusammenfassung . . . . . . . . . . . . . . . . 123

\begin{tabular}{ll}
\hline Resumé und Ausblick & 127
\end{tabular}

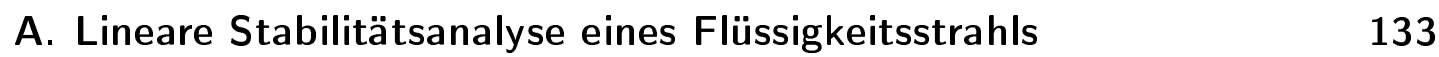

B. Druckabfall entlang eines Rohres mit kreisförmigem Querschnitt 139

\begin{tabular}{|l|l|}
\hline C. Impedanz des piezokeramischen Schallwandlers & 141
\end{tabular}

D. Einfluss der Düsengeometrie auf die Austrittsgeschwindigkeit $\begin{array}{ll}\text { und die Fitparameter } C_{1} \text { und } C_{2} & 143\end{array}$

\begin{tabular}{ll}
\hline E. Schaltpläne der Hochspannungselektronik zur Tropfenaufladung & 147
\end{tabular}

F. Überblick der PIV-Messungen zum Tropfeneinschlag auf ein rotierendes Substrat 153

\begin{tabular}{lr}
\hline Symbolverzeichnis & 167
\end{tabular}

\begin{tabular}{ll}
\hline Literaturverzeichnis & 178
\end{tabular} 


\section{Einleitung}

Seit mehr als einem Jahrhundert ist ein wachsendes wissenschaftliches Interesse an Tropfen und deren Aufprall auf die verschiedensten Substrate zu verzeichnen, wohl auch durch die Fotografien von Worthington [1] ausgelöst. Mittels äußerst kurzer Belichtungszeiten gelang es ihm vor gut 100 Jahren erstmals Aufnahmen zu zeigen, welche die Details und Ästhetik während des Auftreffens von Tropfen veranschaulichen konnten (siehe Abb. 0.1). Durch die Fortschritte in der Hochgeschwindigkeitsphotographie und der computergestützten Simulation der Strömungsdynamik war es möglich, ein stetig tieferes Verständnis der involvierten physikalischen Prozesse zu erlangen. Dabei wurden insbesondere folgende Themenbereiche untersucht: die Morphologie während des Einschlags, wie z.B. Deformation und Ausbreitung auf dem Substrat |2 9 , ,,fingering“ $\mid 10-\sqrt{12}$, ,,splashing“ |13 19], ,rebound“ |20|, ,sideway jetting“ 21-23|, der Einschlag auf einen Flüssigkeitsfilm |24-26|, der Ursprung und die Entwicklung einer Gasschicht zwischen dem Tropfen und dem Substrat 27-31], aber auch schließlich die Erzeugung der Tropfen selbst 32,33$]$. Herausforderung ist hier die Skalierung und die Reproduzierbarkeit von Tropfengröße und -geschwindigkeit sowie die Genauigkeit von Aufprallort und -zeitpunkt. Insbesondere sehr kleine (im Mikrometerbereich) und sehr schnelle $\left(\sim 100 \mathrm{~m} \mathrm{~s}^{-1}\right)$ Tropfen stehen hier im Fokus des Interesses, u.a. wegen ihrer Anwendung in der Oberflächenreinigung. Dies ist auch der Hintergrund der vorliegenden Arbeit.

Ein gebräuchlicher Mechanismus der Tropfenerzeugung ist der Zerfall eines größeren Flüssigkeitsfilaments in kleinere Fragmente [34-37], wie z.B. beim Zerfall eines aus einer Düse austretenden Flüssigkeitsstrahls. Rayleigh [38] untersuchte bereits zum Ende des 19. Jhd. den Zerfallsprozess eines zylinderförmigen Flüssigkeitsstrahls in Tropfen durch Kapillarwellen und externe Störungen, welche den Strahl unter Einwirkung der Oberflächenspannung [39] instabil machen. In den 1960er Jahren konnte gezeigt werden, dass sich diese inhärenten Instabilitäten nutzen lassen, um den Strahlzerfall extern angeregt in eine Kette gleichgroßer (im Weiteren als „,monodispers“ bezeichneter) Tropfen zu steuern 40,41. 


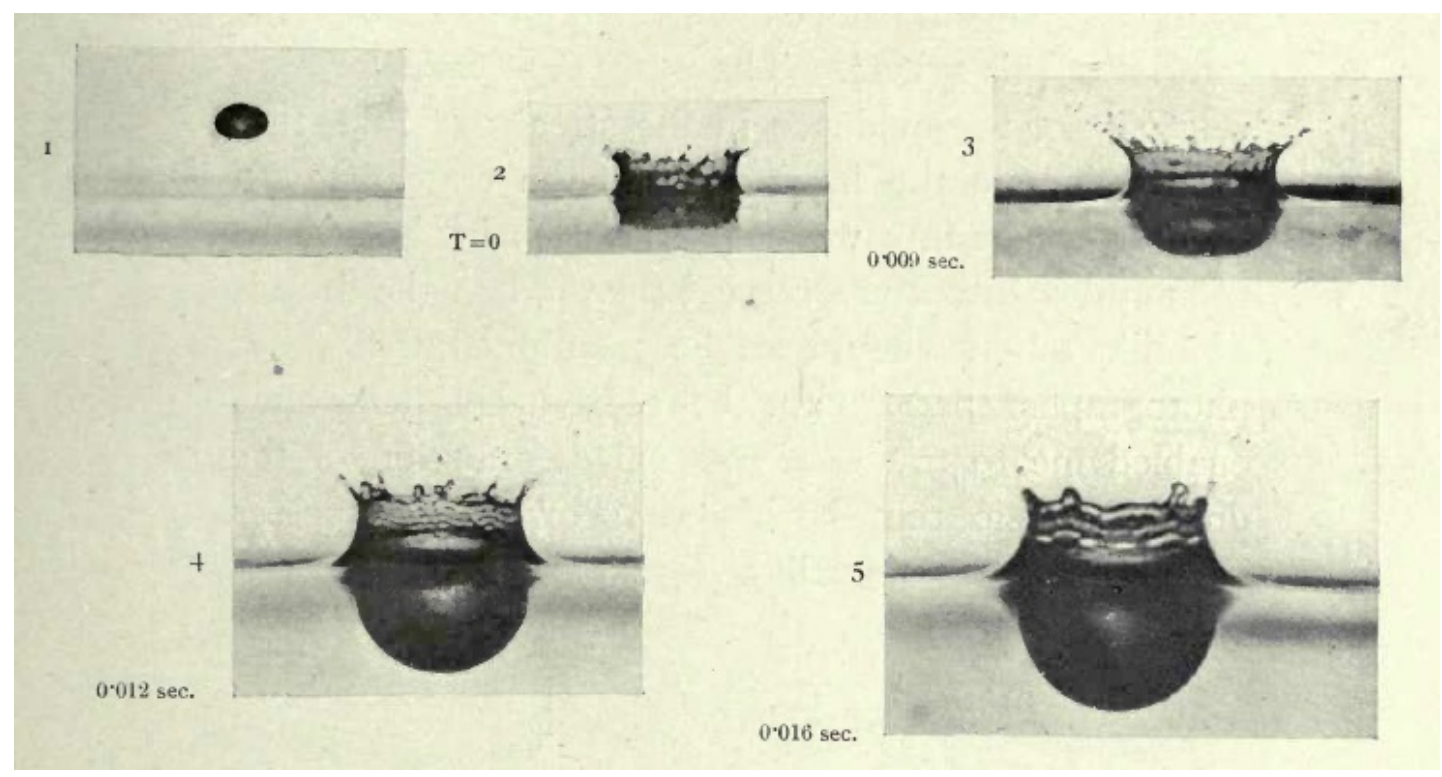

Abbildung 0.1: Aufnahme vom Einschlag eines Tropfens in ein tiefes Flüssigkeitsreservoir durch Worthington [1], Kap. IV, S. 71. Es ist die Formation eines Einschlagkraters und einer Korona mit wegspritzenden Tröpfchen zu beobachten.

Hohe Genauigkeit bei der Erzeugung von Tropfen und Sprays ist ein wichtiger Bestandteil vieler moderner Anwendungen wie z.B. Tintenstrahldrucker [42-44], „rapid prototyping“ [45], Aerosol-Standards für Kalibrierungszwecke [46, 47], „needle-less injection“ [48], Kraftstoffeinspritzung [49-51], ,cold spray deposition “ 52 , , ,plasma-spraying“ $53 \mid$ und Reinigungsanwendungen von sensiblen Halbleiteroberflächen $554-56$. Bei einem Blick in die Literatur zeigt sich jedoch, dass während sich ein Großteil der Untersuchungen mit langsamen $\left(\sim 1 \mathrm{~m} \mathrm{~s}^{-1}\right)$, millimetergroßen Tropfen beschäftigt, eine Vielzahl der zuvor genannten Anwendungen in einem beinahe „komplementären“ Parameterbereich liegt, nämlich, wie oben erwähnt, schnelle ( $>10 \mathrm{~m} \mathrm{~s}^{-1}$ ), mikrometergroße Tropfen.

So kommen in der letztgenannten Anwendung (Reinigung von Halbleiteroberflächen) Hochgeschwindigkeitssprays zum Einsatz, welche kleinste Verunreinigungen abreinigen können [57-59|, jedoch auch teilweise strukturellen Schaden auf dem Substrat verursachen können $60-63$. . Für derartige Prozesse ist es mit der Zeit immer schwieriger geworden, die richtigen Parameter aus Tropfengröße und -geschwindigkeit zu finden und einzuhalten, bei welchen eine möglichst vollständige Reinigung ohne gleichzeitige Erosion erzielt werden kann [64, 65. Dies liegt in den immer kleiner werdenden Strukturgrößen begründet, deren strukturelle Festigkeit immer weiter abnimmt. 


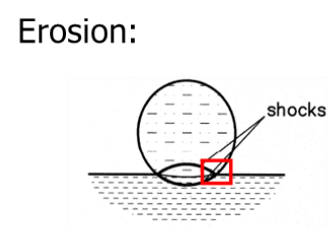

Reinigung:
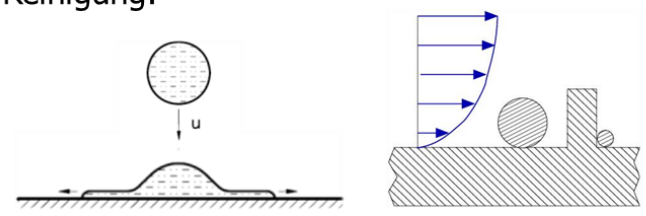

(a)

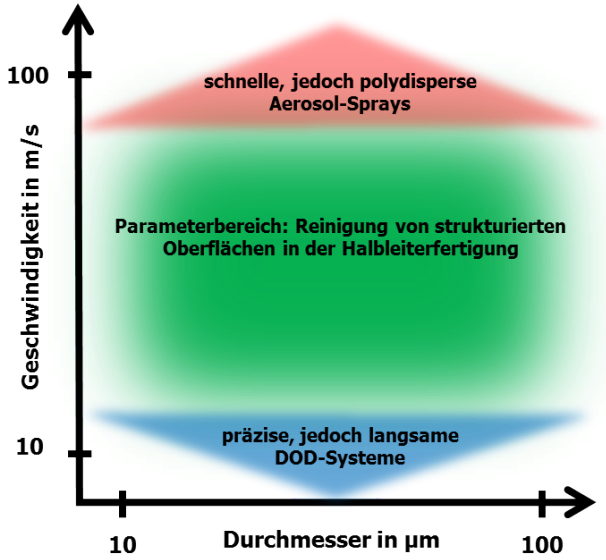

(b)

Abbildung 0.2: (a): Für das Verständnis der Reinigungswirkung beim Tropfenaufprall ist das Zusammenwirken und die Skalierung verschiedener Teilaspekte notwendig: z.B. Erosion durch transiente hohe Drücke und Stoßwellen (,shocks") und Reinigung durch hohe Wandschubspannungen. Teilabbildung (a) stellt diese Aspekte mit den idealisierten Attributen „Erosion“ und „Reinigung“ schematisch dar (mit Genehmigung aus [66] und [67] entnommen). (b): Die häufig verwendeten Methoden Aerosol-Sprays (rot) und DOD-Systeme („Drop-On-Demand“, blau) weisen nur ungenügende Eigenschaften für eine detailliertere Untersuchung im Anforderungsbereich für kontrollierte Reinigung (grün) auf.

Hieraus ergibt sich die Herausforderung, eine Spraymethode zu entwickeln, die eine exakte Kontrolle der Tropfengröße und -geschwindigkeit und damit ein noch detaillierteres Verständnis der relevanten physikalischen Prozesse zulässt [57, 68-70]. In diesem konkreten Beispiel geht es um das in Abb. 0.2a skizzierte Zusammenwirken von erosiven und zur Reinigung beitragenden Prozessen. So kann es beim Tropfeneinschlag auf ein festes Substrat (oder auch eine Flüssigkeit) zu transienten Druckspitzen $\Delta p$ kommen, die näherungsweise proportional zur Einschlagsgeschwindigkeit sind und für den vorliegenden Fall 100 bar und mehr erreichen können $54,60,62,71,75]$. Gleichzeitig entsteht während des Tropfeneinschlags eine radial nach außen gerichtete Flüssigkeitsströmung. Die physikalische Größe Wandschubspannung $\tau$ beschreibt die dadurch auf ein Flächenelement an der Substratoberfläche wirkende Kraft. Diese Kraft wirkt ebenso auf anhaftende Kontaminationen [56,58,76,78, , jedoch ist ihre Abhängigkeit von der Einschlagsenergie der Tropfen oder der Dicke eines Flüssigkeitsfilms auf dem Substrat noch weitgehend unerforscht. 
Die bisher gewählten Herangehensweisen zur Problemuntersuchung schneller Mikrotropfen lassen sich in Bezug auf die Tropfenerzeugung im Wesentlichen auf zwei Methoden reduzieren:

I) Verfahren aus der Drucktechnik, häufig als Inkjet oder DOD („Drop-OnDemand") bezeichnet, ermöglichen die Erzeugung präziser Mikrotropfen verschiedenster Größen. Diese Systeme sind jedoch häufig durch eine niedrige Repetitionsrate und niedrige Tropfengeschwindigkeit limitiert.

II) Durch ein schnell aus einer Düse ausströmendes Gas lässt sich ein AerosolSpray mit sehr schnellen Tropfen erzeugen. Die Größenverteilung der Tropfendurchmesser ist dabei jedoch sehr polydispers.

Auf dieser Grundlage ist die vorliegende Arbeit wie folgt strukturiert:

Kapitel 1 befasst sich mit den Möglichkeiten, den notwendigen Bedingungen und den Begrenzungen einer Methode zur Erzeugung monodisperser Sprays für einen Parameterbereich von Tropfendurchmesser $D=10 \mu \mathrm{m}$ bis $100 \mu \mathrm{m}$ und Tropfengeschwindigkeit $U=10 \mathrm{~m} \mathrm{~s}^{-1}$ bis $100 \mathrm{~m} \mathrm{~s}^{-1}$. Ausgehend von bereits bekannten Grundlagen, geschaffen durch Lindblad und Schneider 40] sowie Brenn [79, soll der akustisch angeregte Zerfall eines Flüssigkeitsstrahls in einem dazu erweiterten Parameterraum untersucht werden. Dabei erweist sich diese Methode als geeignet, um mit hoher Genauigkeit sowohl die Tropfengröße als auch die Tropfengeschwindigkeit zu variieren und über längere Zeiträume zu kontrollieren.

Kapitel 2 beschreibt die Möglichkeit der Erweiterung der zuvor validierten Methode (Erzeugung monodisperser Tropfensprays) zu einer gezielten Präparation eines Einzeltropfens. Durch elektrische Aufladung und Ablenkung eines Mikrotropfens ist es möglich, kontrollierte Experimente zum Aufprall eines Einzeltropfens durchzuführen. Die dabei gewonnenen Erkenntnisse lassen die Folgerung zu, dass, solange die involvierten dimensionslosen Kennzahlen wie z.B. Reynolds-Zahl und Weber-Zahl gleich sind, sich ein Großteil der in der Forschung bisher gewonnenen Erkenntnisse und Formalismen zum Einschlag langsamerer und größerer Tropfen auch auf den Parameterbereich schneller Mikrotropfen anwenden lässt.

Kapitel 3 überträgt die Erzeugung eines monodispersen Tropfensprays in ein anwendungsbezogenes Beispiel aus der Halbleiterfertigung. Mittels einer zur „Particle Image Velocimetry“ (PIV) ähnlichen Methode wird die durch den Tropfeneinschlag erzeugte substratnahe Strömung auf einem sich drehenden Glaswafer gemessen. In Abhängigkeit verschiedenster Prozessparameter wie z.B. 
Tropfengröße und -geschwindigkeit, Rotationsgeschwindigkeit des Substrats, Dicke des Flüssigkeitsfilms auf dem Substrat, etc. lassen sich die auftretenden Scherströmungen nahe der festen Oberfläche bestimmen. Es werden damit Aussagen zur Optimierung auf eine möglichst hohe Reinigungseffektivität abgeleitet.

Abschließend werden die Ergebnisse im Gesamtkontext diskutiert und ein Ausblick auf mögliche Weiterführungen der vorliegenden Untersuchung gegeben. 



\section{Kapitel 1}

\section{Erzeugung monodisperser Hochgeschwindigkeits-Sprays*}

\subsection{Motivation}

Die gängigen Verfahren zur Sprayerzeugung (z.B. Vernebelung durch einen mitreißenden Luftstrom) weisen eine polydisperse Verteilung sowohl in Bezug auf die Tropfengröße $D$ als auch auf die Tropfengeschwindigkeit $U$ auf. Für eine gezielte Nutzung einer bestimmten Kombination aus $D$ und $U$ soll in diesem Kapitel eine Methode beschrieben werden, die gleichzeitig einen breiten, steuerbaren Parameterraum $D=10 \mu \mathrm{m}$ bis $100 \mu \mathrm{m}$ und $U=10 \mathrm{~m} \mathrm{~s}^{-1}$ bis $100 \mathrm{~m} \mathrm{~s}^{-1}$ abdeckt, als auch eine hohe Genauigkeit bzw. Langzeitstabilität mit Abweichungen dieser Größen von den Zielwerten $<0,5 \%$ aufweist.

Die zur Anwendung gekommene Methode basiert auf einem extern angeregten Plateau-Rayleigh-Zerfall $[37-39,80]$. Hierbei zerfällt ein Flüssigkeitsstrahl, welcher von einer Düse aus in Luft übergeht, innerhalb eines gewissen Bereiches von Reynolds-Zahl Re und Ohnesorge-Zahl Oh in Tropfen.

Mit Augenmerk auf den Mikrometerbereich soll untersucht werden, wie sich dieser Prozess durch Anregung mit einem geeigneten Ultraschallsignal steuern lässt, sodass der Flüssigkeitsstrahl in eine möglichst gleichgroße, sprich monodisperse, Kette von Mikrotropfen zerfällt. Die Geschwindigkeit dieses „Tropfensprays“ kann über Änderung des Volumenstromes durch die Düse dem gewünschten Wert angepasst werden. Im Unterschied zur oft üblichen Auffassung eines ,Sprays“ besitzt dieser Spezialfall der monodispersen und gleich schnellen Tropfenkette keinen divergenten Austrittswinkel aus der Düse. Durch Anlegen elektrischer Felder kann allerdings die Flugrichtung der Tropfen gezielt geändert werden, was später in Kap. 2 behandelt wird. 
Der theoretische Hintergrund der Methode wird zunächst in Kap. 1.2 erläutert, gefolgt von der Beschreibung der verwendeten experimentellen Methoden in Kap. 1.3. In Kap. 1.4 werden die erzielten Resultate dargestellt und diskutiert. Eine Zusammenfassung der Ergebnisse folgt in Kap. 1.5.

\footnotetext{
${ }^{*}$ Dieses Kapitel ist zu Teilen veröffentlicht in Frommhold et al. „High-speed monodisperse droplet generation by ultrasonically controlled micro-jet breakup" (Experiments in Fluids, 55, 2014) 81 und Okorn-Schmidt, Frommhold et al. „Particle Cleaning Technologies to Meet Advanced Semiconductor Device Process Requirements" (ECS Journal of Solid State Science and Technology, 3, 2014) [56].
} 


\subsection{Grundlagen}

\subsubsection{Lineare Stabilitätsanalyse}

Der Zerfall eines Flüssigkeitsstrahls in Tropfen soll mit Hilfe der linearen Stabilitätsanalyse durch das Zerfallskriterium eines endlos ausgedehnten flüssigen Zylinders untersucht werden. Hierfür wird der Strahlradius $r$ über eine gewöhnliche Differentialgleichung der Form $\mathrm{d} r / \mathrm{d} t=f(r)$ an einem Fixpunkt $R$ betrachtet, $f(R)=0$. Der Gleichgewichtszustand wird mit einer kleinen Störung $\tilde{r}$ überlagert, $r(t)=R+\tilde{r}$. Ob diese Störung über die Zeit anwächst oder zum Gleichgewicht zurückkehrt, lässt sich mittels der Zeitableitung

$$
\frac{\mathrm{d} \tilde{r}}{\mathrm{~d} t}=\frac{\mathrm{d}}{\mathrm{d} t}(r-R) \stackrel{R=\text { konst. }}{=} \frac{\mathrm{d} r}{\mathrm{~d} t}=f(r)=f(R+\tilde{r})
$$

überprüfen. Eine anschließende Taylor-Entwicklung ergibt

$$
f(R+\tilde{r})=f(R)+\tilde{r} \cdot \frac{\mathrm{d} f(R)}{\mathrm{d} r}+\mathcal{O}\left(\tilde{r}^{2}\right) \quad .
$$

Terme höherer Ordnung werden für $\mathrm{d} f(R) / \mathrm{d} r \neq 0$ vernachlässigt und mit $f(R)=\mathrm{d} R / \mathrm{d} t=0$ erhält man

$$
\frac{\mathrm{d} \tilde{r}}{\mathrm{~d} t} \approx \tilde{r} \cdot \frac{\mathrm{d} f(R)}{\mathrm{d} r}
$$

Dieser Ausdruck lässt sich mit einem Exponentialansatz $\tilde{r} \sim \exp (\mathrm{d} f / \mathrm{d} r \cdot t)$ und Überlagerungen davon lösen und soll nun auf einen inkompressiblen, nichtviskosen Flüssigkeitszylinder in Luft übertragen werden, siehe Abb. 1.1.

Für Störungen mit der räumlichen Wellenlänge $\lambda$ bzw. der Wellenzahl $k$ entlang des Zylinders lässt sich nach Ausführung der linearen Stabilitätsanalyse (siehe Anh. A) ableiten, dass

$$
|k R|<1 \Rightarrow \frac{2 \pi}{\lambda}<1 \Rightarrow \lambda_{\text {krit. }}>2 \pi R
$$

gilt. Somit sind Flüssigkeitsstrahlen aller Durchmesser/Radien (unabhängig von der Dichte und Oberflächenspannung der Flüssigkeit) instabil, sobald die Wellenlänge der Störung größer als der Zylinderumfang ist. Allerdings wachsen die Störungen unterschiedlich schnell an. In Gl. A.15 aus Anh. Alässt sich erkennen, dass es eine maximal instabile Wellenlänge gibt. Es ist im Experiment zu erwarten, dass diese maximal instabile Wellenlänge den Zerfallsprozess in Tropfen dominieren wird. 


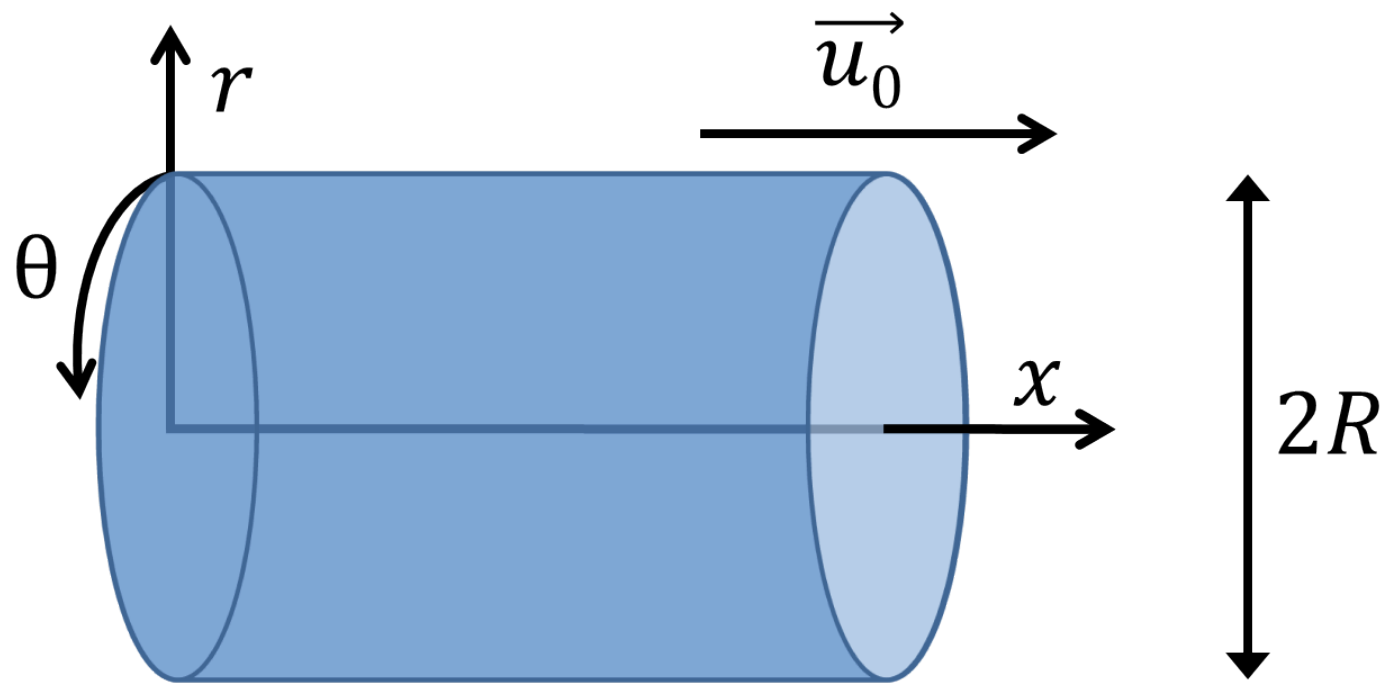

Abbildung 1.1: Abschnitt des unendlich ausgedehnten und ungestörten Flüssigkeitszylinders mit Radius $R$ und Geschwindigkeit $u_{0}$ entlang der $\mathrm{x}-$ Achse.

\subsubsection{Akustisch gesteuerter Zerfall eines Flüssigkeitsstrahls}

In der vorliegenden Untersuchung wird ein Flüssigkeitsreservoir mit einem Überdruck $\Delta p$ beaufschlagt. Als Resultat wird die enthaltene Flüssigkeit durch eine nur wenige Mikrometer große, runde Öffnung (im Weiteren als Düse bezeichnet) entweichen. Es kann angenommen werden, dass der resultierende Flüssigkeitsstrahl einen Durchmesser $d$ besitzt, der näherungsweise dem Düsendurchmesser entspricht. Des Weiteren wird der Strahl durch die dynamische Viskosität $\mu$, die Dichte $\rho$, die Oberflächenspannung $\sigma$ und die Austrittsgeschwindigkeit $U$ (als Resultat des Überdrucks) beschrieben. Nach Austritt aus der Düse wird der Strahl

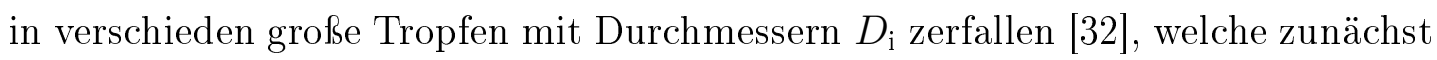
die gleiche Geschwindigkeit $U$ besitzen.

Wie häufig in der Literatur gebräuchlich wird im Weiteren die Notation der dimensionslosen Wellenzahl $k a=\pi d / \lambda<1$ gewählt (mit dem Strahlradius $R=a=d / 2$ ). Die in Anh. A analytisch hergeleitete Bedingung für den Strahlzerfall besagt, dass nur solche räumlichen Störungen mit Wellenlängen $\lambda>\pi d$ zur Tropfenbildung führen. Die Wachstumsrate jeder Mode ist hier jedoch unterschiedlich. Somit ist zu erwarten, dass nicht alle Wellenzahlen in einer vorgegeben Zeit schnell genug anwachsen können. Empirisch wurde gezeigt 41, 79, 82, dass ein stabiler Zerfall in monodisperse Tropfen gewährleistet ist, wenn für die Wellenzahl gilt $0,3 \lesssim k a \lesssim 0,9$. Bei Auftragung der Wachstumsrate entsprechend 


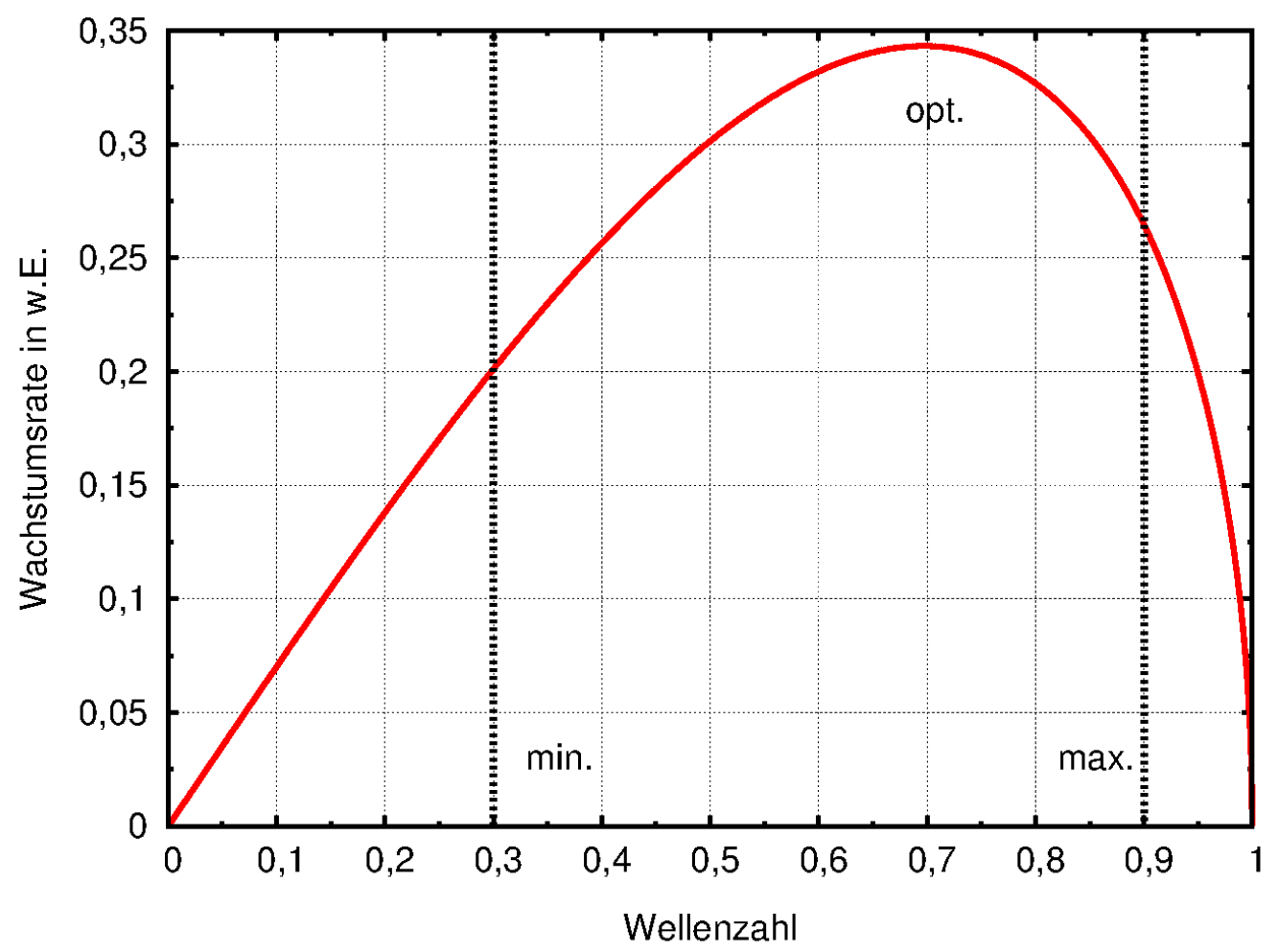

Abbildung 1.2: Die Wachstumsrate als Funktion der Wellenzahl zeigt ein Maximum bei etwa $k a \approx 0,7$. Dieser Arbeitspunkt erweist sich aufgrund des schnellen Wachstums einer Störung als „optimal“ für die Erzeugung monodisperser Tropfen. Experimentell [79] erwies sich der Bereich zwischen „minimal“ $k a \approx 0,3$ und „maximal“ $k a \approx 0,9$ als hierfür ebenfalls benutzbar (durch gestrichelte Linien gekennzeichnet).

Gl. A.15 aus Anh. A wird ersichtlich, dass selbige außerhalb dieses Bereiches einen kleineren Wert aufweist, siehe Abb. 1.2. Insbesondere ist ein Maximum bei $k a \approx 0,7 \mathrm{zu}$ sehen. Eine detailliertere Beschreibung der mikroskopischen Vorgänge beim Zerfallsprozess sind z.B. in [35, 37, 38] zu finden.

Während Störungen der verschiedensten Wellenlängen in einem realen System immer stochastisch vorhanden sind, ist es möglich, zusätzlich extern Störungen mit einer im Vergleich dazu größeren Amplitude aufzuprägen. So lassen sich z.B. durch Anlegen einer zeitlich variablen Spannung an einen piezokeramischen Schallwandler elektrische Signale in mechanische/akustische Vibrationen umwandeln, die so den Zerfallsprozess anregen. Für eine feste Frequenz $f$ (hier im kHz-Bereich) wird der Flüssigkeitsstrom an der Düse moduliert und die Störungen breiten sich näherungsweise mit der Strahlgeschwindigkeit $U$ aus. Die räumliche Wellenlänge lautet entsprechend $\lambda=U / f$ und die dimensionslose 
Wellenzahl $k a=\pi d f / U$ 1 . Im Falle eines genügend schnellen Anwachsens der Störung entstehen Tropfen von identischem Durchmesser mit einem untereinander konstanten Abstand $\lambda$.

Weitere Überlegungen folgen in Kap. 1.4. wo genauer auf die Bestimmung der nutzbaren Frequenzen zur Erzeugung monodisperser Tropfen eingegangen wird. Der Einfluss der verwendeten Flüssigkeit und der Zusammenhang zwischen Überdruck und Austrittsgeschwindigkeit aus der Düse werden an jener Stelle ebenfalls ausgeführt.

${ }^{1}$ Es gilt zu beachten, dass sich die akustische Wellenlänge der Schallausbreitung $\lambda_{\mathrm{s}}=c / f$ mit der Schallgeschwindigkeit $c$ ausbreitet (longitudinale Schwingung) und dass die räumliche Wellenlänge $\lambda$ die Störungen entlang der Flüssigkeitsstrahls beschreibt (transversale Schwingung der Oberfläche). 


\subsection{Experimenteller Aufbau}

Zur Erzeugung eines monodispersen Tropfensprays wurden zwei verschiedene Konstruktionsweisen für die Düse verwendet: A) eine Edelstahlvariante (siehe Kap. 1.3.1) und B) eine Siliziumvariante (siehe Kap. 1.3.2). Da sich die Funktionsweise teils ähnelt, erfolgt zunächst eine allgemeinere Beschreibung und anschließend eine detailliertere in den entsprechenden Unterkapiteln.

Abbildung 1.3 zeigt einen schematischen Überblick der Methode zur Tropfenerzeugung. Ein druckdichter Behälter wird mit der zu untersuchenden Flüssigkeit gefüllt und über ein Rohr-/Schlauchsystem (hier zum Großteil aus dem Kunststoff PEEK bestehend ${ }^{2}$, TECHLAB GmbH) unter Druck gesetzt. Dies kann z.B. wie in Kap. 1.3.1 mit einer Gasflasche (reiner Stickstoff) oder auch wie in Kap. 1.3.2 mit einer Spritzenpumpe (neMESYS, cetoni GmbH) geschehen!3. Abhängig von Größe bzw. Gestaltung des Flüssigkeitsbehälters und der Flüssigkeitszufuhr können praktisch unbegrenzte Laufzeiten erreicht werden. Der Druck lässt sich in beiden Fällen mittels eines Manometers zwischen 0 bar und 100 bar auf 0,5 bar genau bestimmen ${ }^{4}$. Ist das System unter Druck gesetzt, entweicht die Flüssigkeit in Form eines feinen Strahls durch die nur wenige Mikrometer große Bohrung/Düse, welche in Größe und Geometrie variabel bzw. austauschbar ist. Die benutzten Düsendurchmesser lagen dabei zwischen etwa $4 \mu \mathrm{m}$ und $27 \mu \mathrm{m}$. Hauptsächlich kamen in der Variante A eine Düse von etwa 19,9 um und in der Variante B eine Düse von etwa 16,8 $\mu \mathrm{m}$ zur Verwendung, welche der Einfachheit halber nachfolgend als $20 \mu \mathrm{m}$ und $17 \mu \mathrm{m}$ Düsen bezeichnet werden.

Für die akustische Anregung kamen piezokeramische Schallwandler (PI Ceramic $\mathrm{GmbH}$ ) verschiedener Größen zum Einsatz. Sie wurden mittels leitendem Zweikomponentenkleber in der Nähe der Düse befestigt. Das notwendige elektrische (sinusförmige) Signal wurde von einem Frequenz-Generator (FG 120, Yokogawa) und Verstärker (HSA 4101, NF Corporation) mit einer maximalen Spannung von bis zu $V_{\mathrm{p}-\mathrm{p}}= \pm 70 \mathrm{~V}$ erzeugt. Durch Versuche mit Schallwandlern verschiedener Geometrie und Größe zeigte sich, dass die genaue Wahl sowie die Resonanzfrequenz nicht kritisch für die Tropfenerzeugung war. Schon geringe Störungen auch außerhalb der Schallwandlerresonanz $2^{5}$ (aber innerhalb des zulässigen Frequenzbereiches) ergaben eine ausreichende Anregung zum gewünschten regulären Zerfall

2 Dieser Kunststoff weist eine hohe Druckfestigkeit auf. Außerdem lösen sich keine Partikel aus dem Material, die die Düsenöffnung verstopfen könnten.

3 Ein Einfluss des höheren Gasgehalts in der Flüssigkeit bei Verwendung von Druckluft konnte in keinem der Experimente beobachtet werden.

4 In Anh. B wird der zu erwartende Druckabfall durch die Rohrverbindungen diskutiert.

5 Der frequenzabhängige Impedanzverlauf des Schallwandlers ist in Anh. C beschrieben. 


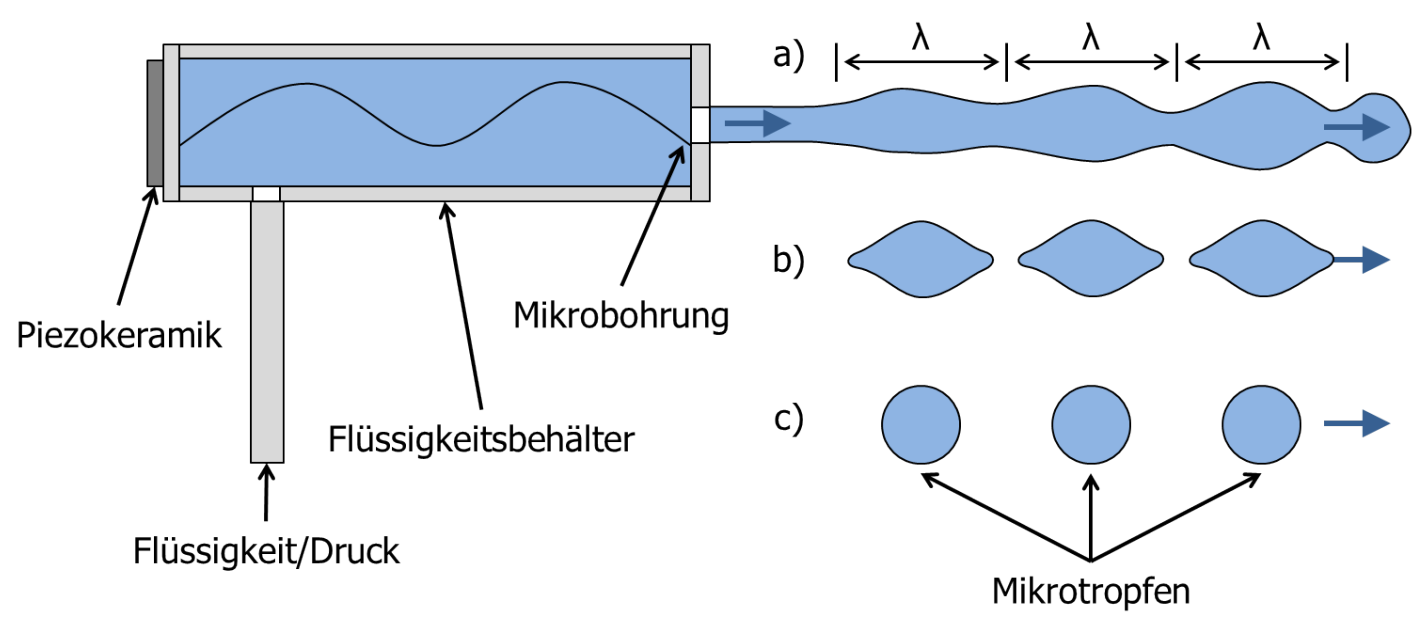

Abbildung 1.3: Schematische Beschreibung der Tropfenerzeugung (nicht maßstabsgetreu). Ein Behälter wird unter Druck mit Flüssigkeit befüllt. a): Durch eine wenige Mikrometer große Öffnung entweicht die Flüssigkeit in Form eines Strahls. Ein piezokeramischer Schallwandler, der in der Nähe der Öffnung angebracht ist, überträgt mechanische/akustische Vibrationen auf den Behälter und damit auch auf den Flüssigkeitsstrahl. b): Der Strahl zerfällt in eine Kette von Fragmenten mit einem untereinander konstantem Abstand $\lambda . c$ ): Aufgrund der Oberflächenspannung entstehen aus den Fragmenten monodisperse runde Mikrotropfen.

in monodisperse Tropfen ergab. Nur im Grenzbereich des Übergangs zwischen monodispersem und polydispersem Zerfall war eine stärkere Abhängigkeit von der Störungsamplitude gegeben (siehe Kap. 1.4.3).

Bildaufnahmen des Zerfallsprozesses wurden durch eine Hochgeschwindigkeitskamera (Photron Fastcam SA5) und unter Verwendung von Bildraten bis zu 100 kfps („fps“, englisch für „Bilder pro Sekunde“) und Belichtungszeiten ermöglicht. Ein Mikroskop-Objektiv mit großem Arbeitsabstand (K2, Infinity Photo-Optical GmbH) und verschiedenen Vergrößerungen (CF1 und CF4) ermöglichten dabei eine ausreichende räumliche Aufösung bei gleichzeitig ausreichendem Abstand zum Flüssigkeitsstrahl. Als Beleuchtungsquelle diente eine weiße $50 \mathrm{~W}$ LED. Der typische Bildausschnitt lag meist im Bereich von $0 \mathrm{~mm}$ bis $50 \mathrm{~mm}$ unterhalb der Düse. Beispielhafte Aufnahmen des freien und des akustisch kontrollierten Zerfallsprozesses sind in Abb. 1.4 dargestellt.

${ }^{6}$ Die typische relevante Zeitskala für den Ablöseprozess eines Tropfens vom Flüssigkeitsstrahl lässt sich durch $\tau \sim \sqrt{\rho a^{3} / \sigma}$ abschätzen, siehe [32], und beträgt $\tau \approx 4 \mu$ sür den Fall von Wasser und einem Düsendurchmesser von $20 \mu \mathrm{m}$. Dies veranschaulicht die Notwendigkeit sehr kurzer Belichtungszeiten. In ausgewählten Experimenten wurde eine spektroskopische Belichtungsquelle mit Belichtungszeiten von $\approx 10$ ns verwendet. 


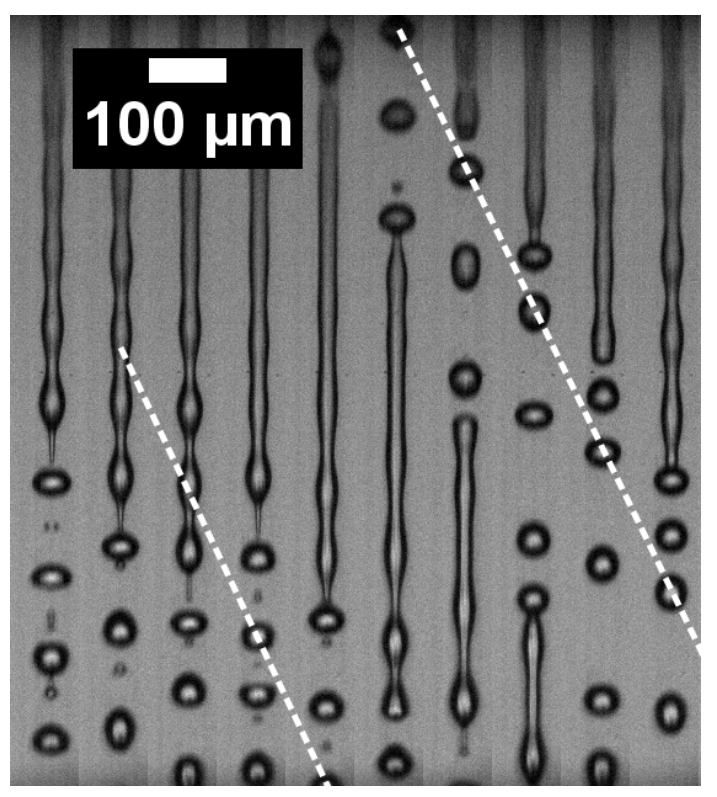

(a) ohne akustische Anregung

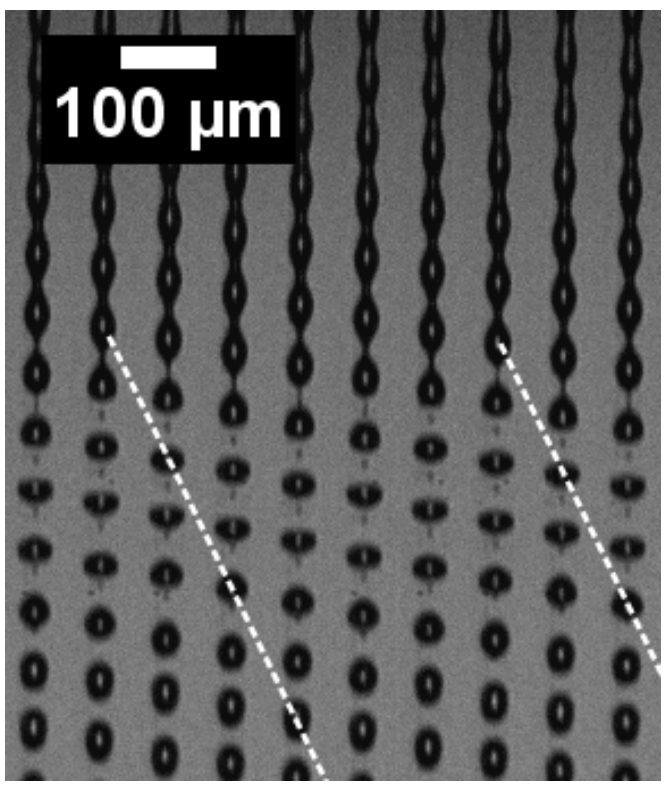

(b) mit akustischer Anregung

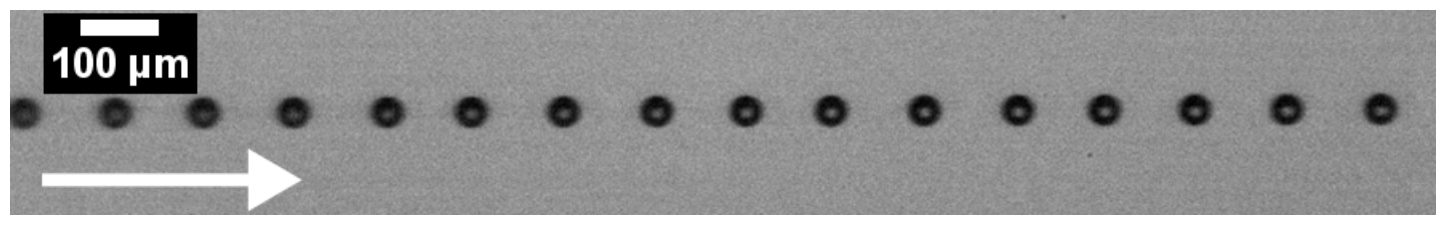

(c) kontrollierte monodisperse Tropfenkette

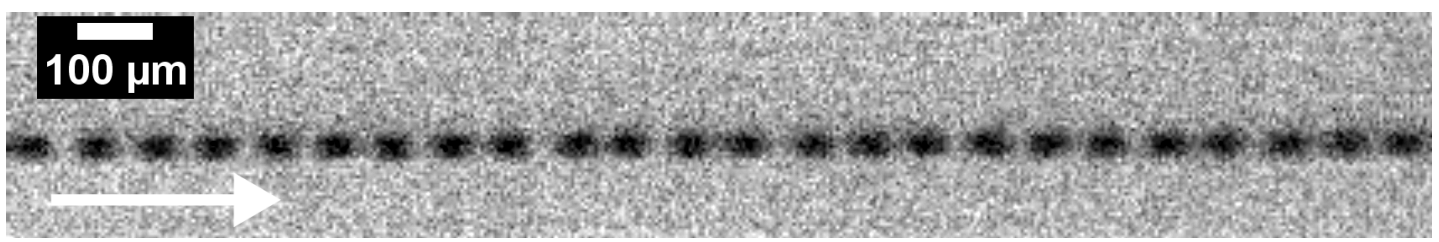

(d) kontrollierte monodisperse Tropfenkette

Abbildung 1.4: Hochgeschwindigkeitsaufnahmen unterhalb der Düse. Die nebeneinander gezeigten Zustände des Flüssigkeitsstrahls in (a) und (b) haben jeweils einen zeitlichen Abstand von etwa $7 \mu$ s (Bildrate: $141 \mathrm{kfps}$, Belichtungszeit: $369 \mathrm{~ns}$, Strahlgeschwindigkeit: $\approx 26 \mathrm{~m} \mathrm{~s}^{-1}$ ). In (a) zerfällt der Flüssigkeitsstrahl frei und ohne akustische Anregung. Der Zerfallsprozess wird kontrolliert und die Verteilung der Tropfengröße wird monodispers, sobald die akustische Anregung aktiviert wird, siehe (b). Weiße gestrichelte Linien dienen zum besseren Nachvollziehen der Bewegung eines individuellen Tropfen, welcher sich von Bild zu Bild nach unten bewegt. Nach Relaxation der Tropfenform ist die finale Tropfenkette etwa $10 \mathrm{~mm}$ unterhalb der Düse gezeigt für: (c) $D \approx 42 \mu \mathrm{m}$ und $U \approx 12 \mathrm{~m} \mathrm{~s}^{-1}$ und (d) $D \approx 32 \mu \mathrm{m}$ und $U \approx 117 \mathrm{~m} \mathrm{~s}^{-1}$. In beiden Fällen verläuft die Bewegung der Tropfen von links nach rechts (entsprechend dem weißen Pfeil). 
Tabelle 1.1: Verwendete Flüssigkeiten und ihre physikochemischen Parameter.

\begin{tabular}{llll} 
Flüssigkeit & $\rho$ in $\mathrm{kg} \mathrm{m}^{-3}$ & $\mu$ in Pas & $\sigma$ in N m \\
\hline DI Wasser & 998 & $1 \times 10^{-3}$ & $72 \times 10^{-3}$ \\
2-Propanol & 780 & $1,9 \times 10^{-3}$ & $22 \times 10^{-3}$ \\
Aceton & 790 & $3,1 \times 10^{-4}$ & $23 \times 10^{-3}$ \\
Ethylenglykol & 1113 & $16,1 \times 10^{-3}$ & $48 \times 10^{-3}$ \\
1-Hexanol & 814 & $4,6 \times 10^{-3}$ & $26 \times 10^{-3}$ \\
1-Butanol & 805 & $2,5 \times 10^{-3}$ & $26 \times 10^{-3}$ \\
\hline
\end{tabular}

Für die Mehrzahl der Experimente wurde gefiltertes, deionisiertes (DI) Wasser (spezifischer, elektrischer Widerstand $\approx 18,2 \mathrm{M} \Omega \mathrm{cm}$ ) verwendet, insbesondere für alle Experimente mit Geschwindigkeiten über $40 \mathrm{~m} \mathrm{~s}^{-1}$. Um die allgemeine Funktionalität der Methode zu bewerten, wurden zusätzlich noch 2-Propanol, Aceton, Ethylenglykol, 1-Hexanol und 1-Butanol verwendet. Die Eigenschaften der Flüssigkeiten sind in Tab. 1.1 aufgelistet.

\subsubsection{Variante A: Edelstahlscheibe}

Als erstes Konstruktionsprinzip der Düse wurde eine Edelstahlvariante (Günther Frey GmbH \& Co. KG), ähnlich zu der von Brenn $|79|$ gewählt, da sie eine hohe mechanische Robustheit und leichte Handhabbarkeit aufweist. Es handelt sich dabei um eine austauschbare Edelstahlscheibe von $2 \mathrm{~mm}$ Dicke und $50 \mathrm{~mm}$ Durchmesser, die über M6-Schrauben mit einem Edelstahlbehälter verbunden ist (siehe Abb. 1.5). Im Zentrum dieser Scheibe befindet sich eine kegelförmige Bohrung von etwa $2 \mathrm{~mm}$ Durchmesser, welche kurz vor dem Durchstoß in einen zweiten Kegel von etwa $100 \mu \mathrm{m}$ mündet, welcher wiederum etwa $5 \mu \mathrm{m}$ vor dem tatsächlichen Durchstoß endet. Mittels einer laserunterstützten Bohrtechnik wurde ein Loch von der anderen Seite genau an der Kegelspitze erzeugt, sodass die eigentliche Düse schlussendlich eine Länge von etwa $5 \mu \mathrm{m}$ und einen Durchmesser von etwa $20 \mu \mathrm{m}$ besitzt.

Wie in Abb. $1.6 \mathrm{zu}$ sehen, ist der Fertigungsprozess mangelhaft. Insbesondere in der Nähe der Düse sind erheblich Materialablagerungen und Fertigungsrückstände zu erkennen. Jedoch schienen während der Versuchsdurchführung diese Mängel keinen signifikanten Einfluss auf die Funktionalität der Düse zu haben. Ein späterer Vergleich mit einem erheblich idealeren/ebeneren Düsendesign zeigte, dass dies die Durchflusseigenschaften und insbesondere die Tropfenerzeugung nur minimal beeinflusst. 


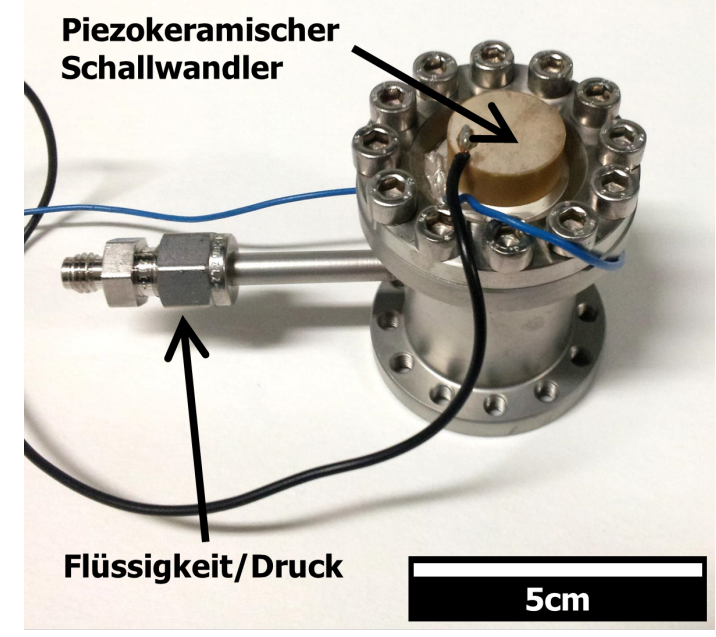

(a)

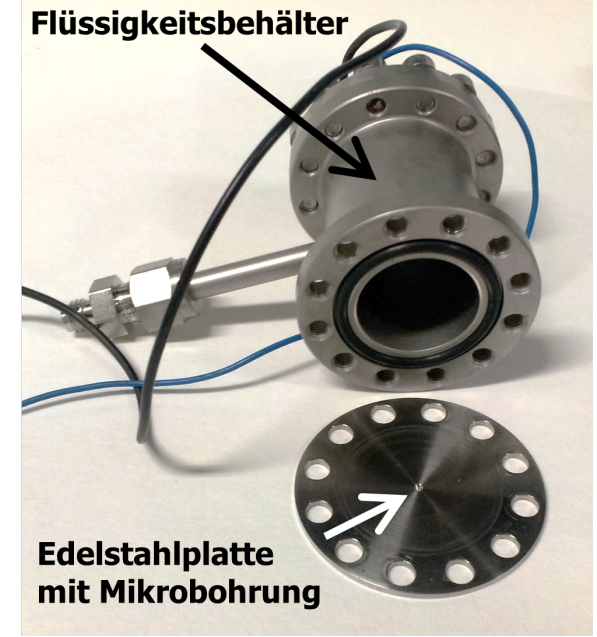

(b)

Abbildung 1.5: Fotos des Flüssigkeitsbehälters mit Zuflussanschluss, des piezokeramischen Schallwandlers und der Edelstahlscheibe mit Mikrobohrung.

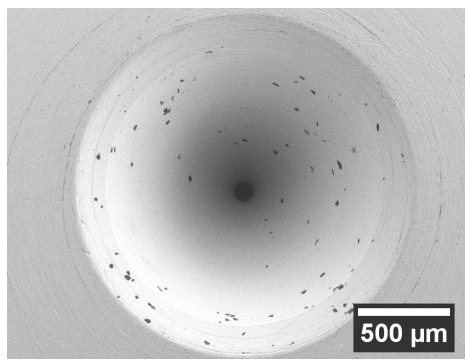

(a) großer Kegel (innen)

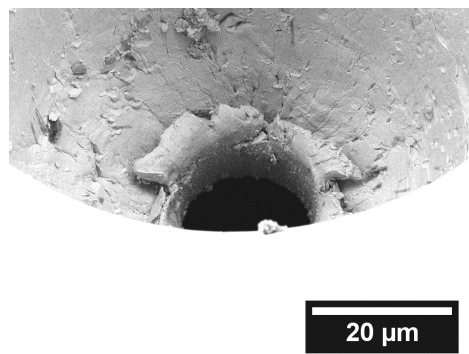

(d) Düse (innen, schräg)

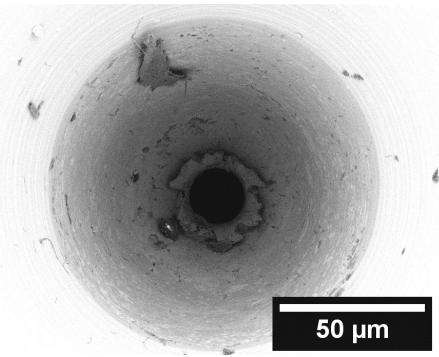

(b) kleiner Kegel (innen)

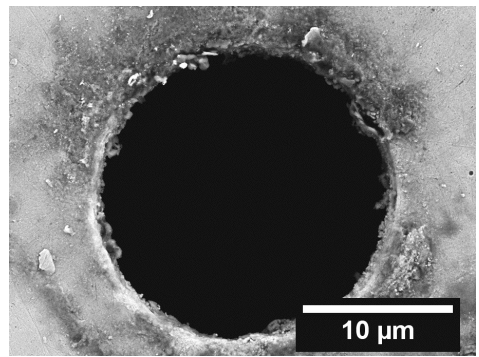

(e) Düse (außen)

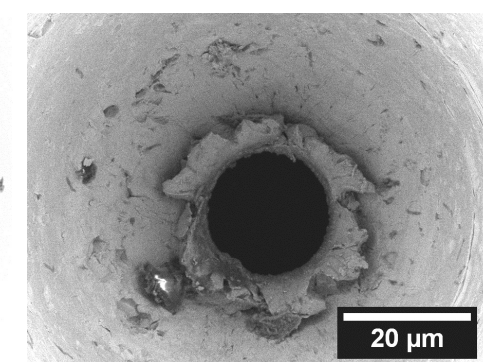

(c) Düse $\sim 20 \mu \mathrm{m}$ (innen)

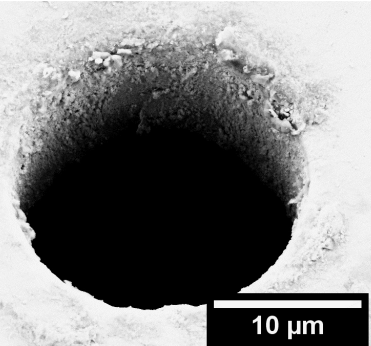

(f) Düse (außen, schräg)

Abbildung 1.6: Detailansicht der $20 \mu \mathrm{m}$ Edelstahldüse aufgenommen mittels „Scanning Electron Microscopy“ (SEM). Die oberen drei Teilabbildungen zeigen in zunehmender Vergrößerung die Gestaltung der Innenseite der Düse. Darunter sind eine leicht schräge Aufnahme der Innenseite und zwei Aufnahmen der Außenseite der Düse gezeigt. Die sichtbaren Verunreinigungen und Materialablagerungen sind auf den Fertigungsprozess zurückzuführen. 


\subsubsection{Variante B: Siliziummembran}

Die im Verlauf mit der Edelstahldüse gewonnenen Erfahrungen wurden in einem nächsten Schritt auf eine Siliziumdüse übertragen. So beeinträchtigte z.B. eine Kontamination der Flüssigkeit $t^{7}$ die Funktionsweise der Edelstahldüse erheblich, da sich typische Verunreinigungen (z.B. Staubkörner) festsetzen können und den Strahl blockieren. In Zusammenarbeit mit einem Hersteller für Mikrosystemtechnik wurde eine auf Silizium basierende Düse entwickelt. Vor der eigentlichen Düse befindet sich ein gesinterter Filter, sodass die Gefahr einer Verstopfung der Mikroöffnung deutlich reduziert ist. Der typische Lochdurchmesser der Düse beträgt zwischen $4 \mu \mathrm{m}$ und $27 \mu \mathrm{m}$. Jedoch ist die eigentliche Düse lediglich $1 \mu \mathrm{m}$ dick, was durch eine spezielle Ätztechnik ermöglicht wurde. Der Vorteil dieses Designs liegt in einer deutlich glatteren Düsengeometrie und der Kompaktheit. Die Düsenform ist damit weniger einem Kanal ähnlich, sondern mehr einem Loch in einer Membran. Der kompakte Siliziumchip befindet sich in einer leicht austauschbaren Kunststoffkappe, siehe Abb. 1.7, welche über weitere Bauteile mit dem druckdichten Flüssigkeitsbehälter verbunden ist.

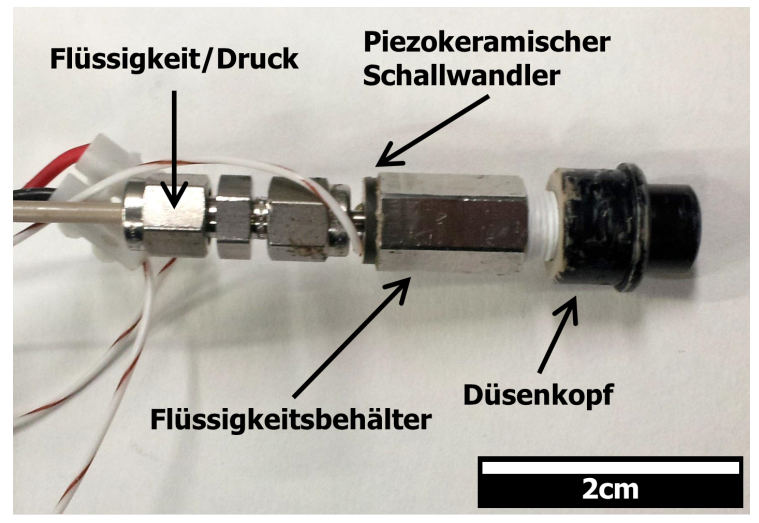

(a)

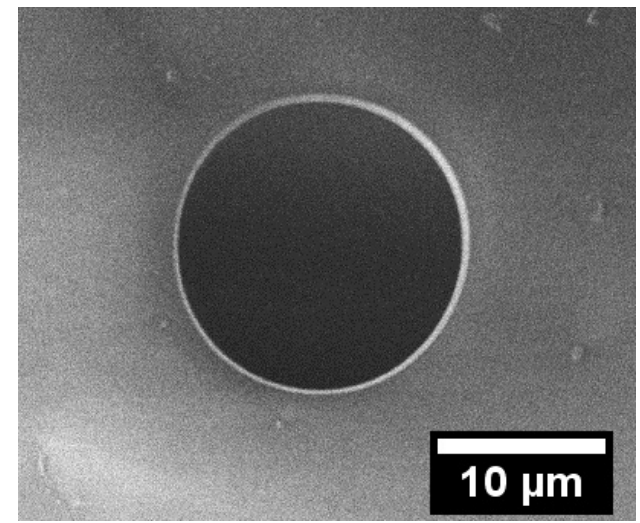

(b)

Abbildung 1.7: (a): Foto des Flüssigkeitsbehälters mit Zuflussanschluss, des piezokeramischen Schallwandlers und des Düsenkopfs. (b): Detailansicht der $17 \mu \mathrm{m}$ Siliziumdüse, aufgenommen mittels „Scanning Electron Microscopy 4 (SEM). Die Öffnung weist eine glatte Kreislinie auf.

7 Alle verwendeten Flüssigkeiten waren hochrein und für den Laborbedarf ausgezeichnet. Das Düsensystem und die Dichtungsringe mussten jedoch häufig geöffnet werden z.B. zum Austausch der Düse, Auffüllen der Flüssigkeit etc. Trotz größter Vorsicht ließen sich gelegentliche Verunreinigungen nicht vollständig vermeiden.

8 Die leichte Verzerrung in Teilabbildung (b) rührt durch elektrische Aufladungen an der Düse her, da das Düsengehäuse aus Kunststoff besteht und damit nicht elektrisch leitfähig ist. 


\subsection{Auswertung}

\subsubsection{Strahlparameter für verschiedene Flüssigkeiten}

Bevor auf die Tropfenerzeugung und deren Kontrollparameter eingegangen wird, sollen zunächst die Eigenschaften des ungestörten Flüssigkeitsstrahls, welcher in verschieden große Tropfen zerfällt, untersucht werden. Dies wird für das Verständnis der Kontrolle der Tropfengröße und -geschwindigkeit von Bedeutung sein.

Es kann angenommen werden, dass nahe der Düse die mittlere Strahlgeschwindigkeit und die Geschwindigkeit der entstehenden Tropfen nahezu identisch ist. Abweichungen, verursacht durch die Abbremsung durch Luftreibung, betragen im betrachteten Bildausschnitt unterhalb der Düse nur wenige Prozent (siehe Kap. 2.3.2. Jedoch ist es möglich, dass es im weiteren Verlauf unterhalb der Düse zu Kollisionen und Koaleszenz kommt (siehe Kap. 1.4.5).

Als Resultat des angelegten Überdrucks $\Delta p$ setzt ein spezifischer Volumenstrom $Q(\Delta p, d, \mu, \rho)$ durch die Düse ein. Im Allgemeinen ist die Durchflussmenge abhängig von dem Druck $\Delta p$, dem Düsendurchmesser $d$, der Viskosität der Flüssigkeit $\mu$ und der Dichte der Flüssigkeit $\rho$. Obwohl die Oberflächenspannung die eigentliche Ursache für den Strahlzerfall ist, ist sie nicht in diesem Ausdruck enthalten [79. Da es sich bei den vorliegenden Düsen näherungsweise um Löcher in dünnen Platten handelt, also ein kleines Aspektverhältnis (Länge durch Durchmesser ist kleiner als eins), kann angenommen werden, dass die Abhängigkeit von der Düsenlänge vernachlässigbar ist.

Brenn [79] leitete mittels Dimensionsanalyse einen Ausdruck für den Volumenstrom her, welcher monoton mit dem Überdruck $\Delta p$ ansteigt. Hierfür wurde der dimensionslose Volumenstrom $Q \rho / \mu d$ und der dimensionslose Druck $\Delta p \rho d^{2} / \mu^{2}$ gewählt. Aufgetragen in einem doppelt-logarithmischen Graphen zeigt sich theoretisch eine lineare Beziehung zwischen beiden Größen. Dies lässt sich ebenfalls im vorliegenden Experiment für alle verwendeten Flüssigkeiten (siehe Tab. 1.1) finden. Ergebnisse für Drücke im Bereich 2 bar bis 10 bar (entsprechend etwa $6 \mathrm{~m} \mathrm{~s}^{-1}$ bis $38 \mathrm{~m} \mathrm{~s}^{-1}$, abhängig von der Flüssigkeit) unter Verwendung der Edelstahldüse sind Abb. $1.8 \mathrm{zu}$ entnehmen. Die Darstellung aller Daten in einem Graphen lässt erkennen, dass sie nahezu auf derselben Geraden liegen. Hiervon ausgehend lässt sich folgende Beschreibung [79 verwenden

$$
\frac{Q \rho}{\mu d}=C_{1} \cdot\left(\frac{\Delta p \rho d^{2}}{\mu^{2}}\right)^{C_{2}} \sim\left(R e_{\mathrm{p}}\right)^{2 C_{2}}
$$

mit den beiden freien Parametern $C_{1}$ und $C_{2}$ bzw. als Funktion der DruckReynolds-Zahl $R e_{\mathrm{p}}=\sqrt{2 \Delta p \rho} \cdot d / \mu$. Die beiden freien Parameter lassen sich mit 


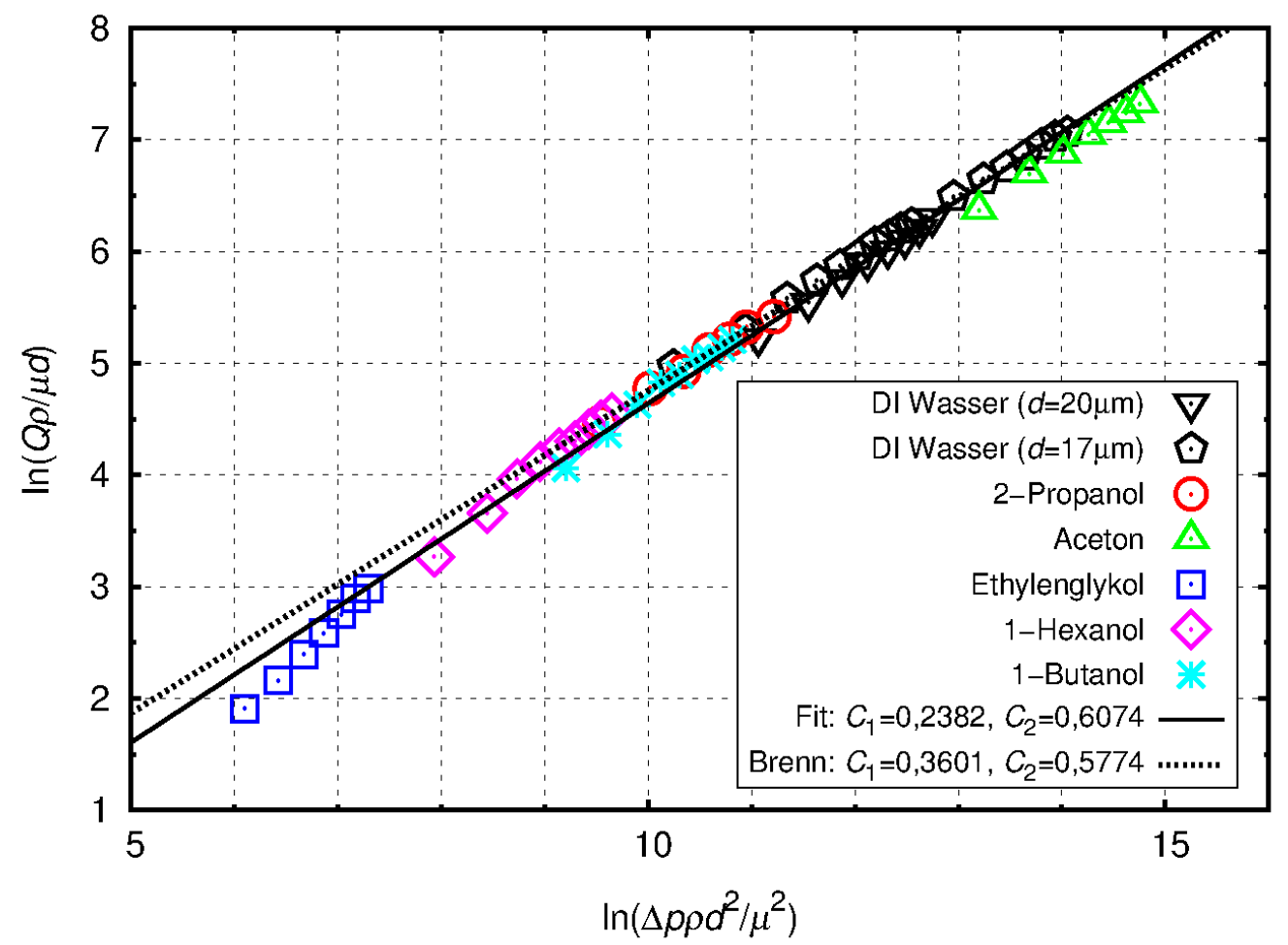

Abbildung 1.8: Darstellung aller Daten für die $20 \mu \mathrm{m}$ Edelstahldüse und aller verwendeter Flüssigkeiten für den dimensionslosen Volumenstrom als Funktion des dimensionslosen Überdrucks. Zusätzlich sind die Daten der $17 \mu \mathrm{m}$ Siliziumdüse für DI Wasser eingetragen. Ein Fit über alle Datenpunkte ist entsprechend Gl. (1.1) (durchgezogene Linie) gezeigt und lässt sich mit den Ergebnissen von Brenn [79 (gestrichelte Linie) vergleichen. Der angelegte Überdruck wird für die $17 \mu \mathrm{m}$ Düse zwischen 2 bar und 45 bar und für die $20 \mu \mathrm{m}$ Düse zwischen 2 bar und 10 bar variiert.

einem linearen Fit über alle Datenpunkte bestimmen. Die resultierende Gerade $\left(C_{1}=0,2382\right.$ und $\left.C_{2}=0,6074\right)$ ist zusammen mit einem Fit von Brenn $\left(C_{1}=0,3601\right.$ und $\left.C_{2}=0,5774\right)$, der auf der Verwendung von Wasser und 2-Propanol basiert, eingezeichnet. Während zunächst eine genügende Beschreibung der Messwerte im doppelt logarithmischen Plot in Abb. 1.8 gegeben zu sein scheint, so lassen sich doch Abweichungen bis zu 30 \% (z.B. für Ethylenglykol) und ähnlich auch für den Fit von Brenn erkennen. Eine individuelle Betrachtung der Flüssigkeiten und Anpassung mit Gl. (1.1) ergab eine höhere Übereinstimmung, typischerweise mit Abweichungen von nur wenigen Prozent. Es kann also davon ausgegangen werden, dass die Werte von $C_{1}$ und $C_{2}$ nicht „universal“ sind, sondern vielmehr abhängig von den Eigenschaften der Flüssigkeit sind. Ein Vergleich der resultierenden Werte $C_{1}$ und $C_{2}$ für die Flüssigkeiten zeigt eine annähernd 


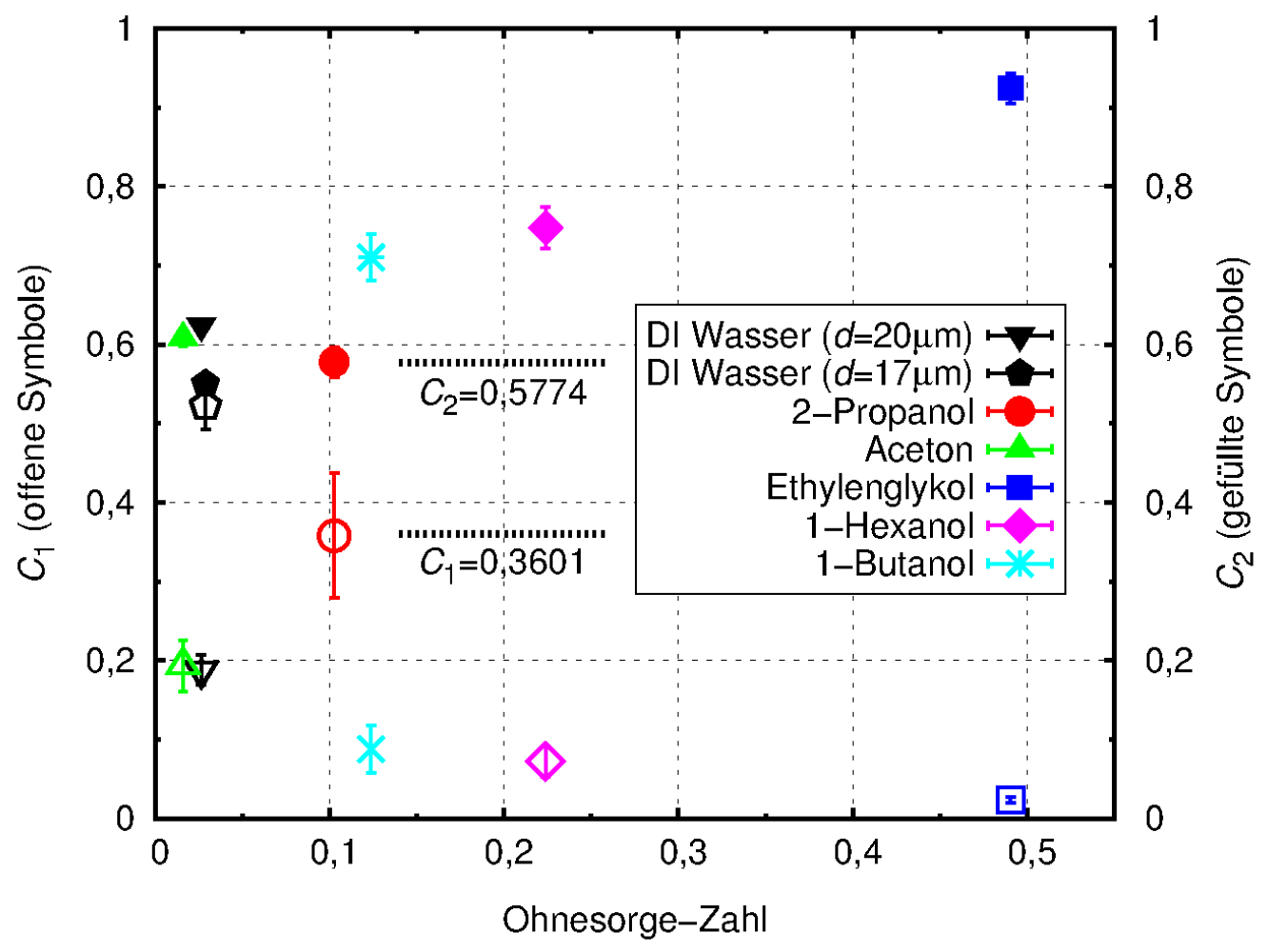

Abbildung 1.9: Überblick der sich ergebenden Fitparameter $C_{1}$ (offene Symbole) und $C_{2}$ (gefüllte Symbole) für die benutzen Flüssigkeiten unter Verwendung der $20 \mu \mathrm{m}$ Düse und für DI Wasser auch mit der $17 \mu \mathrm{m}$ Düse. Die Ergebnisse von Brenn [79] sind als gestrichelte Linien eingefügt.

monotone Variation mit der Viskosität. Entsprechend ist die Abhängigkeit der Fitparameter von der Ohnesorge-Zahl $O h=\mu / \sqrt{\rho \sigma d}$ in Abb. 1.9 abgebildet. Weitere Untersuchungen haben gezeigt, dass ebenfalls eine Abhängigkeit von $C_{1}$ und $C_{2}$ von der Düse selbst besteht ${ }^{9}$, insbesondere für die verschiedenen Varianten A und B. Experimente mit DI Wasser und einer $17 \mu \mathrm{m}$ Düse führen zu leicht anderen Werten als mit der $20 \mu \mathrm{m}$ Düse. Möglicherweise resultiert dies aus den unterschiedlichen Geometrien, inneren Reibungseigenschaften und Details der tatsächlichen Strömung an sich. Um dies zu veranschaulichen, sind diese Daten ebenfalls in Abb. 1.8 und 1.9 einbezogen. Dennoch kann in allen Fällen der Volumenstrom durch die Düse ausreichend gut mit Gl.11.1 beschrieben werden.

9 In Anhang $\mathrm{D}$ wird anhand einer Finite Elemente Simulation noch einmal gesondert auf den Einfluss der Düsengeometrie im Bezug auf den Durchfluss und die Fitparameter eingegangen. 


\subsubsection{Begrenzung der Tropfengeschwindigkeit}

Die Tropfengeschwindigkeit $U$ (nahe der Düse identisch zur Strahlgeschwindigkeit) kann in erster Näherung aus dem Bernoulli-Prinzip mittels $U=\sqrt{2 \Delta p / \rho}$ abgeleitet werden. Die Umstellung von Gl. (1.1) liefert jedoch die genauere Relation

$$
U=C_{1} \cdot \frac{4 \mu}{\pi \rho d} \cdot\left(\frac{\Delta p \rho d^{2}}{\mu^{2}}\right)^{C_{2}} \sim\left(R e_{\mathrm{p}}\right)^{2 C_{2}}
$$

Abbildung 1.10 zeigt die Geschwindigkeitsmessungen und den Fit der Kurve für die $17 \mu \mathrm{m}$ Siliziumdüse. Konstruktionsbedingt ließen sich mit dieser Düse und mit DI Wasser langzeitstabil deutlich höhere Drücke verwenden als mit der Edelstahldüse, hier bis zu 45 bar. Es zeigt sich eine gute Übereinstimmung zwischen den Messdaten und der mit den ermittelten Parametern $C_{1}=0,5221$ und $C_{2}=0,5503$ bestimmten Kurve. Aufgrund der guten Interpolation der experimentellen Daten lässt sich Gl. (1.2) mit kalibrierten Parametern $C_{1}$ und $C_{2}$ für eine korrekte Vorhersage der Austrittsgeschwindigkeit des Strahls verwenden.

Erwartungsgemäß existiert ein minimal nötiger Druck, um anfänglich einen Flüssigkeitsstrahl zu erzeugen. Da bei sehr niedrigen Drücken die Oberflächenspannung und die Viskosität die kinetische Energie des Strahls überwiegen, würde die Flüssigkeit nur langsam herausfließen. Es würde sich ein größerer Tropfen bilden, welcher sich schließlich wegen seines eigenen Gewichts ablösen und herunterfallen wird. Experimentell untersucht wurde diese Restriktion unter anderem von Walzel [83] und lässt sich als kritische (limitierende) Weber-Zahl $W e=\rho U^{2} d / \sigma$ in Abhängigkeit von der Ohnesorge-Zahl ausdrücken. Walzel fand einen kritischen Wert von $W e_{\text {krit }}=14,5 \cdot O h^{0,08}$, unterhalb dessen sich kein kontinuierlicher Flüssigkeitsstrahl formt.

Die obere Grenze der erreichbaren Geschwindigkeit steht im Zusammenhang der Interaktion mit dem umgebenden Gas (typischerweise Luft). Mit zunehmender Strahlgeschwindigkeit bilden sich vermehrt Störungen in der Scherschicht der umgebenden Luft, weswegen der Einfluss ihrer Viskosität nicht mehr vernachlässigt werden kann. Ohnesorge [84] und Haenlein |49| haben zwei qualitative Übergänge beschrieben. Oberhalb einer bestimmten Geschwindigkeit beginnt der Flüssigkeitsstrahl Instabilitäten aufzuweisen, die als „Zerwellen“ bezeichnet wurden (in der englischsprachigen Literatur auch häufig ,wind-induced regime“). Hier entwickelt der gesamte Strahl eine wellenähnliche Form quer zur Ausbreitungsrichtung. Für noch höhere Geschwindigkeiten zerfällt der Flüssigkeitsstrahl bereits schon sehr nahe der Düse gänzlich in einen feinen Tropfennebel, „Zerstäuben“ genannt (engl. ,atomization“). Ersterer Übergang (zum Zerwellen-Regime) ließ sich 


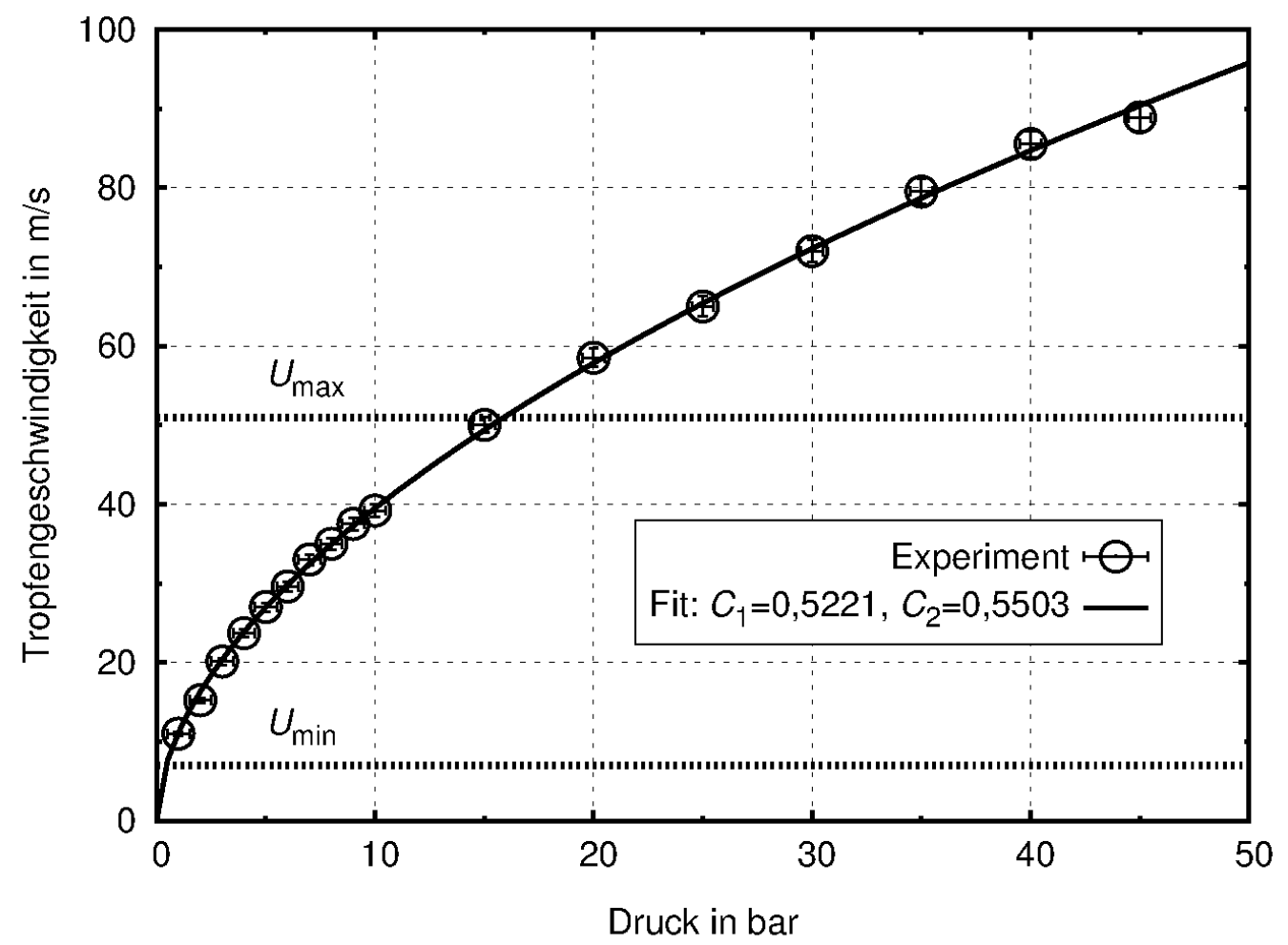

Abbildung 1.10: Der Zusammenhang zwischen Überdruck und resultierender Tropfengeschwindigkeit lässt sich mit guter Übereinstimmung durch Gl. 1.2 beschreiben (durchgezogene Linie). Die beiden freien Parameter $C_{1}$ und $C_{2}$ werden für jede Düse und Flüssigkeit bestimmt, hier für die $17 \mu \mathrm{m}$ Siliziumdüse und DI Wasser. Die gestrichelten Linien zeigen für die verwendete Düse die theoretischen Geschwindigkeitsgrenzen entsprechend [83] und [84] für eine stabile Bildung eines glatten Flüssigkeitsstrahls in Luft.

von Ohnesorge über einen weiten Parameterbereich mittels der Reynolds-Zahl $R e=\rho U d / \mu$ und der Ohnesorge-Zahl beschreiben, wobei er unter normalem, atmosphärischen Druck etwa ab einer kritischen Reynolds-Zahl $R e_{\text {krit }} \approx 133 \cdot O h^{-4 / 5}$ stattfand. Der Übergang zum Zerstäuben wurde von diesen Autoren bei einer Reynolds-Zahl $R e_{\text {krit }} \approx 1000 \cdot O h^{-4 / 5}$ beobachtet.

Die gestrichelten Linien in Abb. 1.10 zeigen die entsprechenden unteren und oberen Grenzen für die $17 \mu \mathrm{m}$ Düse. Ein Vergleich mit den experimentell erreichbaren Geschwindigkeiten zeigt, dass während die untere Grenze ${ }^{10}$ von etwa

10 Der Datenpunkt bei 1 bar markiert in etwa den experimentell minimal möglichen Druck zur Erzeugung eines Flüssigkeitsstrahls. Experimente mit einem Druck etwas unter 1 bar führten zwar auch noch zu einem Flüssigkeitsstrahl, jedoch beträgt der Fehlerbereich des Manometers 0,5 bar. Daher wurde darauf verzichtet, diese Datenpunkt zu berücksichtigen. 


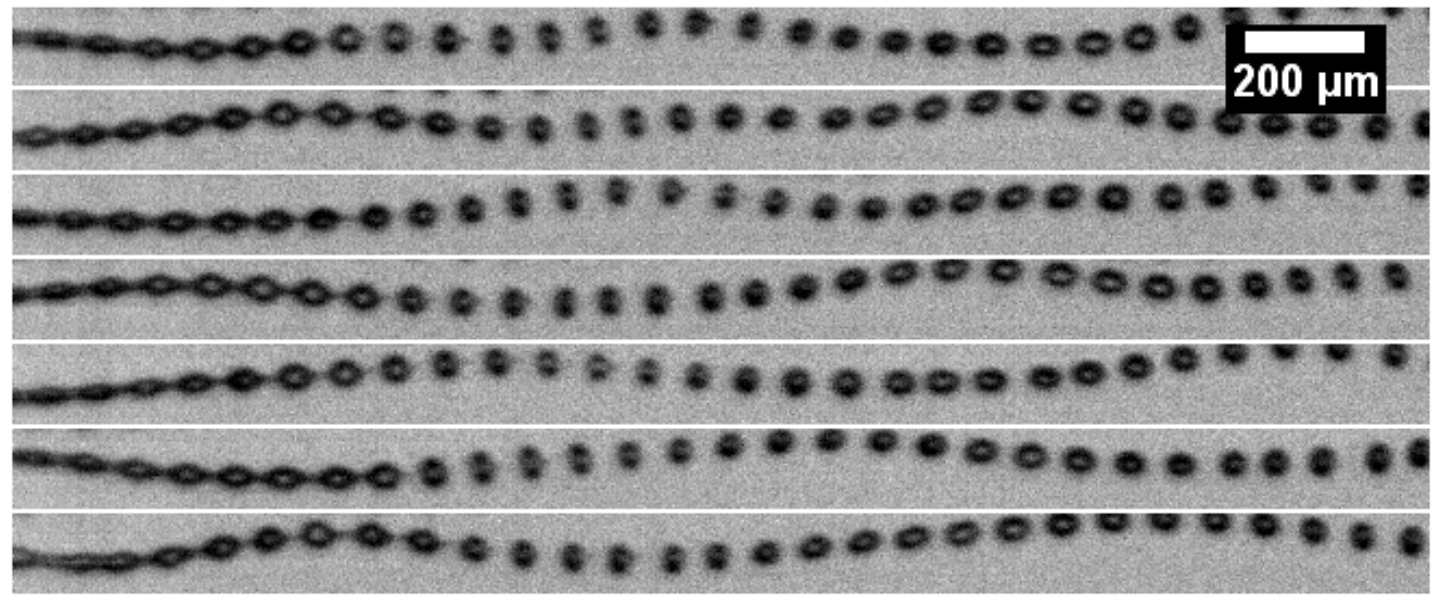

Abbildung 1.11: Hochgeschwindigkeitsaufnahme unterhalb der Düse (Bildrate: 88 kfps und Belichtungszeit: 369 ns). Die Tropfen bewegen sich von links nach rechts (die Bilder sind um $90^{\circ}$ gedreht). Der Flüssigkeitsstrahl verlässt eine $26 \mu \mathrm{m}$ Siliziumdüse bei etwa $47 \mathrm{~ms}^{-1}$, was eine Ohnesorge-Zahl $O h \approx 0.023$ und eine kritische Reynolds-Zahl $R e_{\text {krit }} \approx 950$ ergibt, wobei die eingestellte Reynolds-Zahl $R e \approx 1200$ beträgt. Während andere Experimente mit diesem Düsendurchmesser und dieser Geschwindigkeit bei einer einzelnen Düse einen glatten Flüssigkeitsstrahl zeigten, so führt die Nähe weiterer Strahlen (nicht im Bildausschnitt; identische Strahlparameter; Abstand etwa $150 \mu \mathrm{m})$ zu einem Übergang hin zu einer wellenähnlichen Verformung quer zur Ausbreitungsrichtung. Dennoch war es möglich, den Zerfallsprozess akustisch bei einer Frequenz von $590 \mathrm{kHz}$ in monodisperse Tropfen zu kontrollieren.

$7 \mathrm{~ms}^{-1}(\Delta p \approx 1$ bar $)$ relativ gut nachvollzogen werden kann, die obere Grenze von etwa $51 \mathrm{~ms}^{-1}(\Delta p \approx 16$ bar $)$ um etwa den Faktor zwei überschritten wurde. In einem Einzelexperiment ${ }^{11}$ bei etwa 75 bar konnte bei Geschwindigkeiten von bis zu $120 \mathrm{~ms}^{-1}$ und akustischer Anregung noch ein stabiler Zerfall in monodisperse Tropfen beobachtet werden (siehe Abb. 1.4d). Daraus lässt sich schließen, dass in dem vorliegenden Experiment die Geschwindigkeitsgrenze nicht durch die Zerwellen-induzierte Instabilität gegeben ist, sondern vielmehr durch eine technische Begrenzung des Aufbaus. Die maximale Strahlgeschwindigkeit entspricht einer Reynolds-Zahl von $R e \approx 2000$, was in etwa zweieinhalb mal größer als $R e_{\text {krit }} \approx 850$ ist. Für diesen erweiterten Stabilitätsbereich kann allerdings angenommen werden, dass die Geometrie der Düse, insbesondere die Länge des letzten rohrähnlichen Abschnittes, von großer Bedeutung ist. Es wurde durch die Experimente von McCarthy und Molloy [34 gezeigt und

11 Oberhalb eines Druckes von 50 bar steigt die Gefahr der Zerstörung der Düse. 


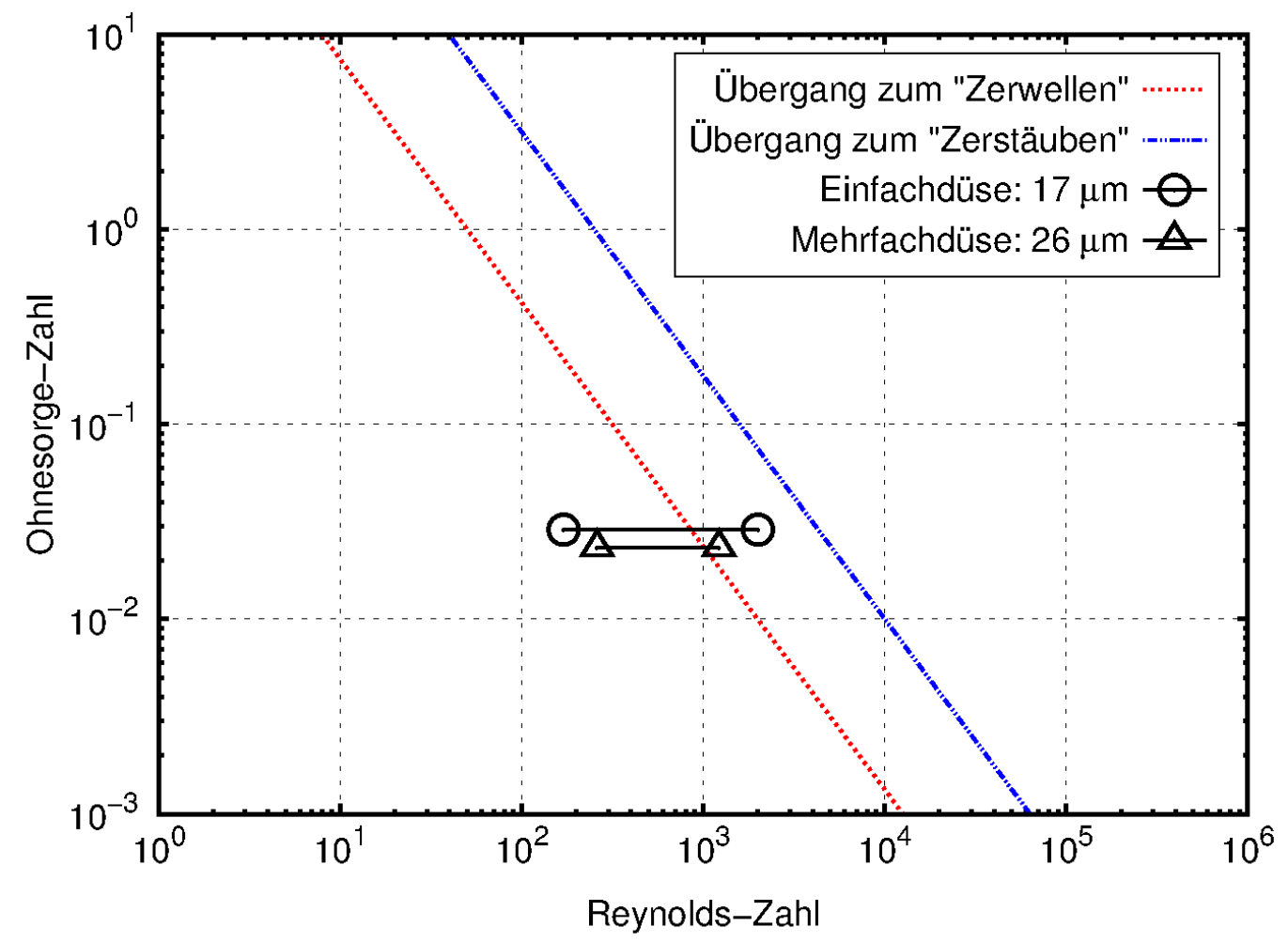

Abbildung 1.12: Erreichter Geschwindigkeitsbereich mit der $17 \mu \mathrm{m}$ Siliziumdüse und der $26 \mu \mathrm{m}$ Siliziumdüse mit mehreren Mikrobohrungen unter Verwendung von DI Wasser, gezeigt als Ohnesorge-Zahl gegen Reynolds-Zahl. Beide Düsen überschreiten die in der Literatur angegebene Grenze zum Zerwellen-Regime. Die Übergänge zum Zerwellen und Zerstäuben sind entsprechend [84] als gestrichelte Linien eingezeichnet.

ebenfalls in 85 beschrieben, dass sich durch Änderung der Düsenlänge die Form des austretenden Flüssigkeitsstrahls zwischen einem „glatten“, stabähnlichen und einem „gestörten“ Zustand ändern ließ. Eine Optimierung der Düsengeometrie für Mikro-Flüssigkeitsstrahlen bzgl. eines möglichst schnellen und gleichzeitig stabilen Strahls scheint möglich [47, wie auch durch das vorliegende Experiment bestätigt. Weitere Informationen über den Strahlzerfall unter Anlegen einer externen akustischen Störung im Zerwellen- und insbesondere im Zerstäuben-Regime würden an dieser Stelle notwendig sein, sind jedoch mit dem vorliegenden Aufbau nicht umzusetzen.

Im Fall von angrenzenden Strahlen (aus weiteren Düsen) zeigte sich eine Querbewegung der gesamten Tropfenkette, die wohlmöglich aufgrund der durch die zusätzlichen Strahlen verursachten Verwirbelungen in der Luft begünstigt wird (siehe Abb. 1.11). Jedoch erfolgte der Zerfall immer noch in monodisperse Tropfen, 
wobei es wahrscheinlich ist, dass die Querbewegung eine Koaleszenz begünstigt. Mithin ist das Vorkommen dieser weiteren Instabilität nicht notwendigerweise eine Limitierung der Methode zur Erzeugung monodisperser Tropfen, bedarf jedoch im konkreten Anwendungsfall einer genaueren Untersuchung.

Abschließend wird in Abb. 1.12 ein Überblick des untersuchten Parameterraums gegeben, ähnlich zu dem in [84] zu findenden Nomogramm. Dieser beinhaltet den Übergang zum Zerwellen und zum Zerstäuben jeweils als diagonale Linien. Die horizontalen Linien mit Symbolen bilden die in den vorliegenden Experimenten erreichten Geschwindigkeiten ab, in dem monodisperse Tropfen erzeugt wurden. Beide Düsenvarianten überschreiten deutlich den Übergang ins Zerwellen, jedoch wiesen sie stets einen stabilen varikosen Strahlzerfall auf.

\subsubsection{Anpassung der Tropfengröße}

Die Tropfengröße ist maßgeblich (scheinbar fest) durch den Düsendurchmesser $d$ vorgegeben, jedoch ist es innerhalb eines bestimmen Frequenzbereiches der externen akustischen Anregung möglich, sie zu beeinflussen. Zusammen mit der Relation $D=\sqrt[3]{3 U d^{2} / 2 f}$ (welche aus der Massenerhaltung folgt) und Umstellung von Gl. (1.2) berechnet sich der Tropfendurchmesser zu

$$
D=\left(\frac{6 C_{1} \mu}{\pi \rho d^{2} f}\right)^{1 / 3} \cdot d \cdot\left(\frac{\Delta p \rho d^{2}}{\mu^{2}}\right)^{C_{2} / 3} .
$$

Unter der Annahme, dass die Anforderungen an die dimensionslose Wellenzahl ka (gegeben in 441,79,82) seine Gültigkeit unabhängig von der Geschwindigkeit hat, sind der minimale und maximale Tropfendurchmesser durch $k a_{\max }$ and $k a_{\min }$ vorgegeben. Für eine $17 \mu \mathrm{m}$ Düse ergibt sich somit $D_{\min }\left(k a_{\max }=0,9\right) \approx 29,5 \mu \mathrm{m}$ und $D_{\max }\left(k a_{\min }=0,3\right) \approx 42,5 \mu \mathrm{m}$, oder anders ausgedrückt eine Variabilität von etwa $-9 \%$ und $+32 \%$ bezogen auf $D_{\text {opt }}\left(k a_{\text {opt }}=0,69\right)=32,3 \mu \mathrm{m}$.

Weiterhin lässt sich aus $k a_{\min }<k a<k a_{\max }$ ableiten, wie sich der mögliche Frequenzbereich mit der Strahlgeschwindigkeit bzw. dem angelegten Überdruck ändert. Da der Druck für eine praxisnahe Fragestellung ebenfalls von Bedeutung ist, werden beide Aspekte in Abbildung 1.13 für einen konkreten Fall dargestellt und mit experimentellen Resultaten belegt. Zur Berechnung der jeweilig zu benutzenden Frequenz des piezokeramischen Schallwandlers in Abhängigkeit von $k a$ lässt sich folgender linearer Zusammenhang finden:

$$
f=k a \cdot C_{1} \cdot \frac{4 \mu}{\pi^{2} \rho d^{2}} \cdot\left(\frac{\Delta p \rho d^{2}}{\mu^{2}}\right)^{C_{2}} .
$$




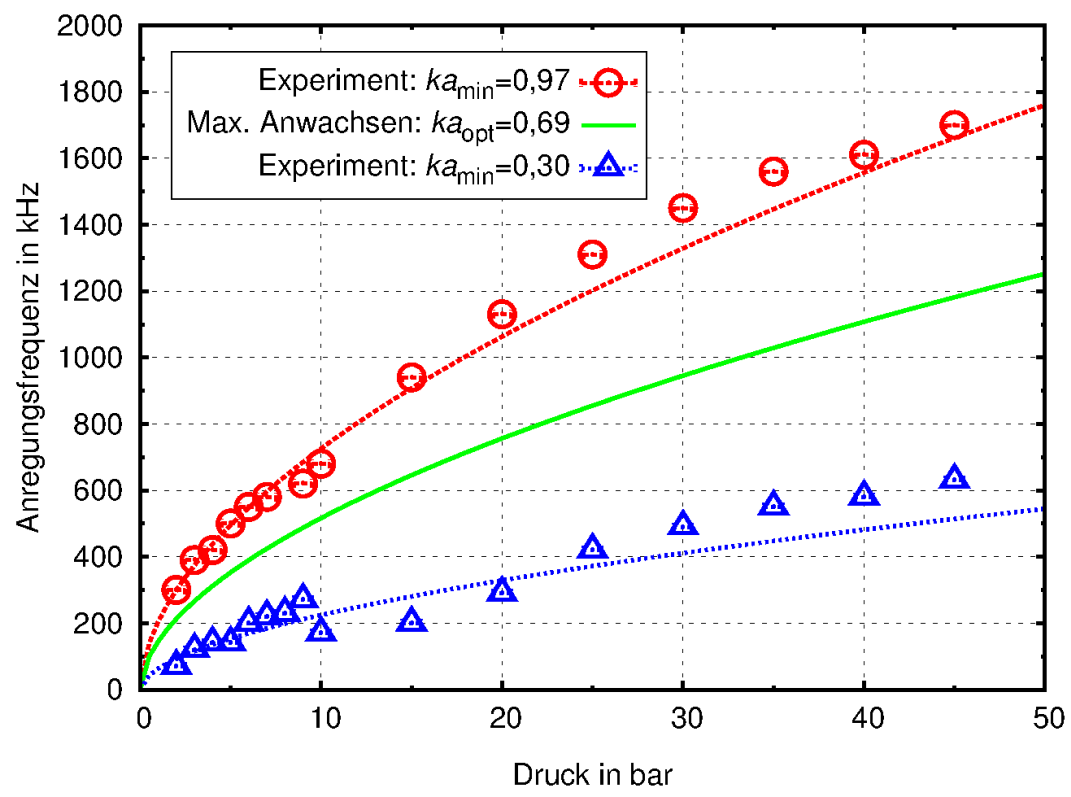

(a)

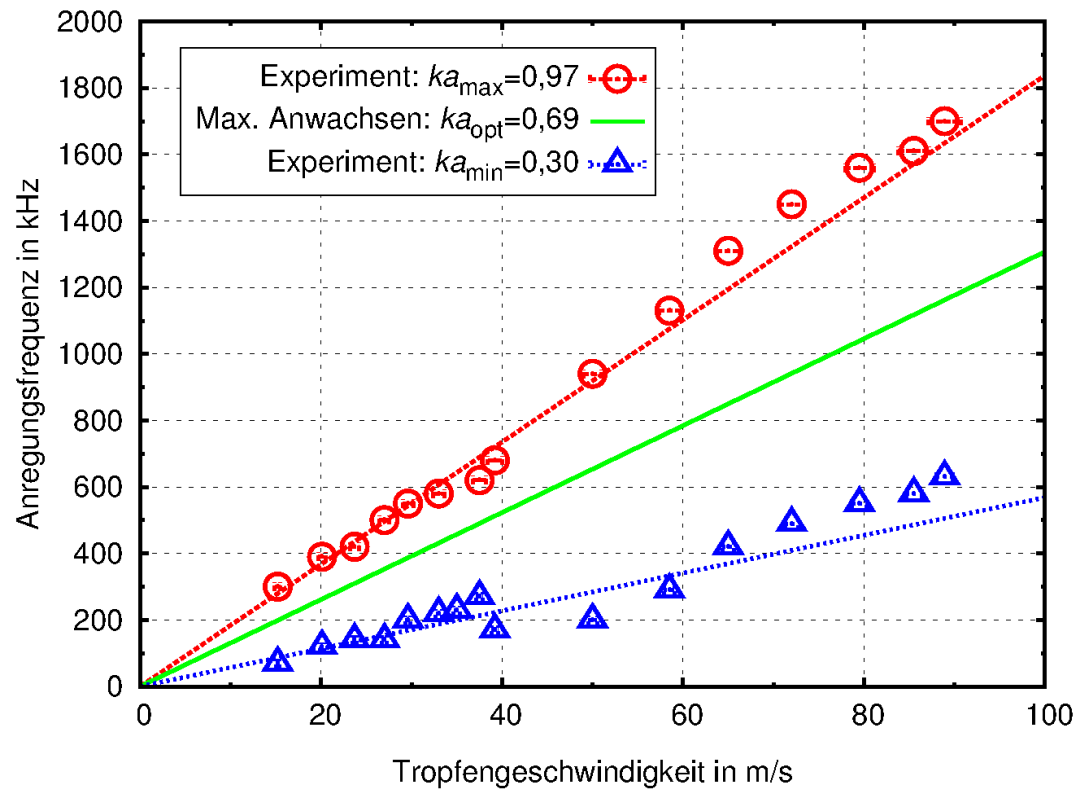

(b)

Abbildung 1.13: Es wurden für die $17 \mu \mathrm{m}$ Düse unter Verwendung von DI Wasser die möglichen Frequenzbereiche für Erzeugung monodisperser Tropfen bei Variation des Überdrucks $\Delta p$ bestimmt. Die experimentell gefundenen Grenzen sind gegen den Druck (a) und gegen die Tropfengeschwindigkeit (b) aufgetragen. Dreiecke markieren die niedrigste und Kreise die höchstmögliche Frequenz, die zum Zerfall in monodisperse Tropfen führte. Innerhalb dieser Grenzen bleibt die Monodispersität stets gewahrt, wogegen außerhalb die Größenverteilung polydispers ist. Die mittlere (durchgezogene) Linie markiert jeweils die Frequenz mit der theoretisch am schnellsten anwachsenden Instabilität. 
Über den gesamten Messbereich von Abb. 1.13 wurde die angelegte Spannung am piezokeramischen Schallwandler konstant gehalten und für jeweils konstante Überdrücke die minimal und die maximal mögliche Frequenz bestimmt. Die Bildung der Tropfen wurde in einem Bildbereich von etwa $5 \mathrm{~mm}$ bis $10 \mathrm{~mm}$ unterhalb der Düse mit einer Hochgeschwindigkeitskamera aufgenommen, wobei der Zerfall in monodisperse Tropfen innerhalb dieses Bereiches geschehen musste.

Es wurde überprüft, ob im gesamten Bereich von $f_{\min }$ bis $f_{\max }$ ein durchgehend monodisperser Zerfall vorzufinden ist. Außerhalb dieses Frequenzbereiches zeigten sich teils recht scharfe Übergänge $(< \pm 10 \mathrm{kHz})$ zu einem polydispersen Zerfall. Die den Frequenzbereich einrahmenden theoretischen Kurven weisen für Drücke $\Delta p<10$ bar (bzw. $U<40 \mathrm{~m} \mathrm{~s}^{-1}$ ) eine gute Übereinstimmung mit den im Experiment gefundenen Bereichen auf. Für höhere Drücke (bzw. Geschwindigkeiten) zeigen sich Abweichungen. Möglicherweise ist die Änderung der Impedanz des piezokeramischen Schallwandlers nicht zu vernachlässigen (vgl. Anh. C), da sich dies bei konstanter Spannung direkt auf die resultierende mechanische Vibration und damit den Strahlzerfall auswirkt. Dennoch ist auch hier eine relativ gute Übereinstimmung mit $0,3<k a<0,9 \mathrm{zu}$ finden, allerdings mit einer Tendenz zu höheren Wellenzahlen $k a_{\max }=0,97$. Zur Orientierung ist jeweils eine durchgezogene Linie in Abb. $1.13 \mathrm{zu}$ finden, welche die Frequenz mit der am schnellsten wachsenden Instabilität bei $k a_{\mathrm{opt}}=0,69$ darstellt, was, wie nachfolgend gezeigt, mit einer verkürzten Zerfallslänge einhergeht.

\subsubsection{Zerfallslänge des Flüssigkeitsstrahls}

Mit ansteigendem Überdruck $\Delta p$ an der Düse steigt auch die Strahlgeschwindigkeit $U$, was wiederum einen Einfluss auf die Zerfallslänge hat, d.h. den Ort der Tropfenablösung vom Flüssigkeitsstrahl. Im Falle eines ungestörten Zerfalls ist diese Länge zeitlich nicht konstant (siehe hierzu auch Abb. 1.4a) und ihre Bestimmung ist durch die Schwankungen entsprechend fehlerbehaftet. Wie schon von Haenlein 49 gezeigt, kommt es zunächst zu einem linearen Anstieg der Zerfallslänge mit der Strahlgeschwindigkeit, da bei angenommen konstanter Zerfallszeit $\tau_{\text {zerf }}$ sich die Störung mit der Strahlgeschwindigkeit ausbreitet. Die Zerfallslänge wächst entsprechend proportional. Diesem Anstieg folgt im Fall von rohr-ähnlichen Düsen ein Maximum 86. Da es sich bei den hier verwendeten Düsen eher um runde Öffnungen in dünnen Platten handelt, ist ein weiterer Anstieg ohne das Erreichen eines Maximums zu erwarten [87, bevor es durch weitere auftretende Instabilitäten zu deutlich komplexerem Verhalten kommen 


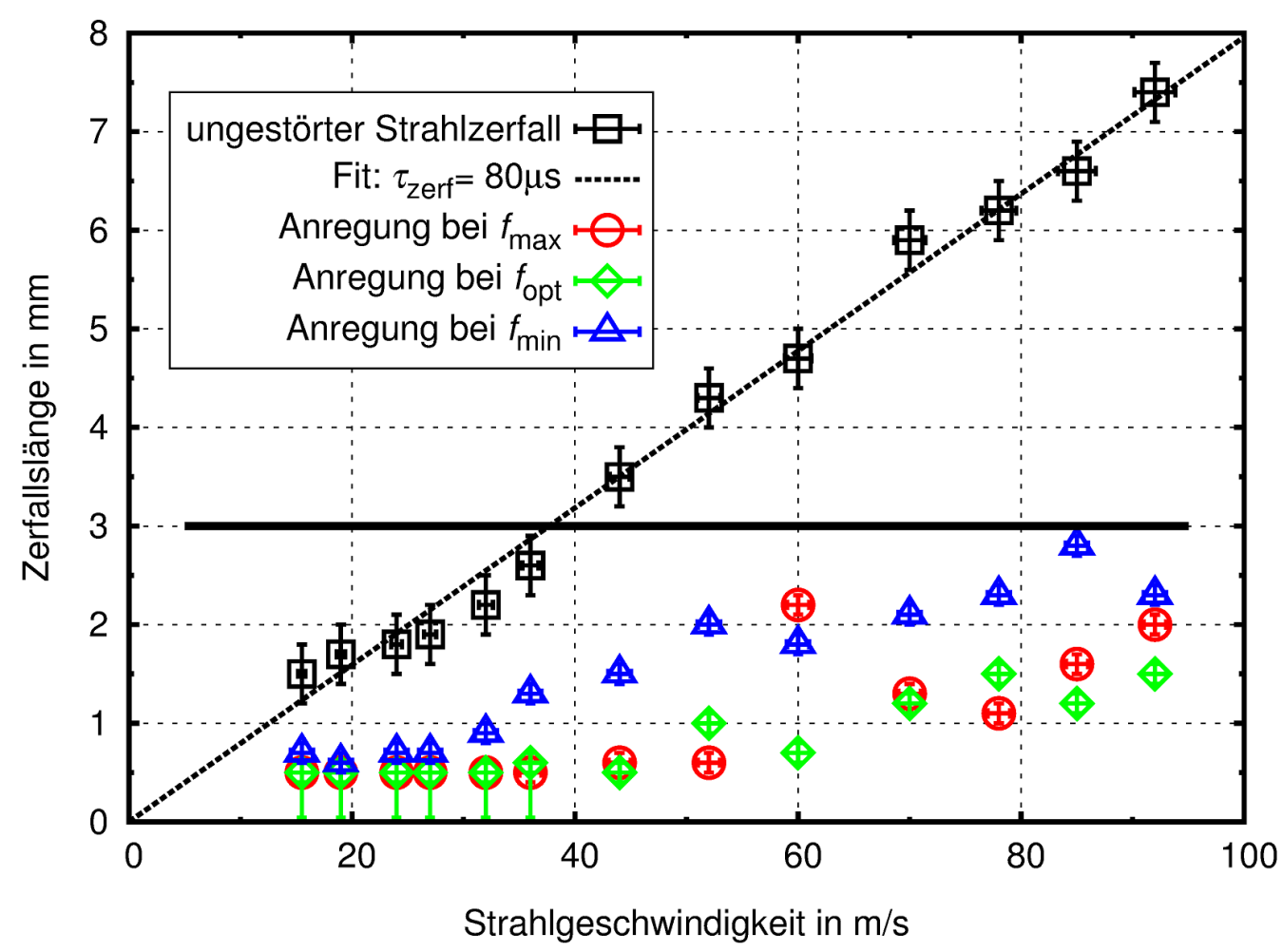

Abbildung 1.14: Bestimmung der Position der Ablösung einzelner Tropfen vom Flüssigkeitsstrahl für die $17 \mu \mathrm{m}$ Siliziumdüse und DI Wasser: ohne akustische Anregung (Quadrate), mit der maximalen (Kreise) und der minimalen (Dreiecke) Frequenz, die zu monodispersen Tropfen führt, und die Frequenz (Rauten), welche dem schnellsten Anwachsen einer Störung entspricht. Ohne akustische Anregung zeigt sich ein linearer Anstieg der Zerfallslänge mit der Strahlgeschwindigkeit. Ein linearer Fit ergibt eine Zerfallszeit von $\tau_{\text {zerf }} \approx 80 \mu \mathrm{s}$ (gestrichelte Linie). Mit akustischer Anregung reduziert sich die Zerfallslänge deutlich und überschreitet $3 \mathrm{~mm}$ nicht (horizontale, durchgezogene Linie).

kann $[85 \mid$. Entsprechend weisen die experimentellen Resultate der $17 \mu \mathrm{m}$ Siliziumdüse in Abb. 1.14 kein Maximum der Zerfallslänge für den ungestörten Fall auf. Ein linearer Anstieg ist deutlich zu sehen, und ein linearer Fit ergibt eine Zerfallszeit von $\tau_{\text {zerf }} \approx 80 \mu \mathrm{s}$.

Durch Überlagerung des Zerfalls mit einer Frequenz innerhalb des funktionalen Frequenzbereiches (und bei konstanter Spannung) lässt sich die Zerfallslänge teils deutlich reduzieren. Abgesehen von einem leichten Anstieg mit zunehmender Geschwindigkeit bleibt die Zerfallslänge stets unterhalb von $3 \mathrm{~mm}$ bezogen auf die Düsenöffnung. Dies gilt sowohl für $f_{\min }$ als auch $f_{\max }$, sprich für die größten als auch kleinsten erzielbaren Tropfendurchmesser. Wie erwartet liegt die Zerfalls- 
länge für $f_{\mathrm{opt}}$, was dem schnellsten Anwachsen einer Störung entspricht, zumeist unter den Ergebnissen mit $f_{\min }$ und $f_{\max }$. Durch eine Erhöhung der angelegten Spannung und damit der akustischen Anregung der Instabilität lässt sich die Zerfallslänge noch weiter verkürzen, teilweise deutlich unterhalb von $1 \mathrm{~mm}$. Die Streuung und Nicht-Monotonie der Daten steht vermutlich, wie zuvor schon beschrieben, im Zusammenhang mit den Resonanzen und der Frequenzabhängigkeit des piezokeramischen Schallwandlers (vgl. Anh. C), was trotz konstanter Amplitude der Wechselspannung zu unterschiedlichen Amplituden der akustischen Anregung führt. Aufgrund der Bauweise des Düsengehäuses war es nicht möglich, Abstände kürzer als 0,5 mm von der Düsenöffnung zu beobachten, weswegen auch noch kürzere Zerfallslängen möglicherweise vorgekommen sind.

\subsubsection{Verteilung der Tropfengröße und -geschwindigkeit}

Für den Fall der $20 \mu \mathrm{m}$ Edelstahldüse, DI Wasser und $U=27 \mathrm{~m} \mathrm{~s}^{-1}$ wurden die Tropfendurchmesser und -geschwindigkeiten mit Bildbearbeitung von Aufnahmen mit einer Hochgeschwindigkeitskamera ausgewertet. Während in erster Näherung die Tropfengeschwindigkeit nahe der Düse der Strahlgeschwindigkeit entspricht, zeigte es sich, dass schon kleine Unterschiede zwischen aufeinanderfolgenden Tropfen im Bezug auf ihre Geschwindigkeit zur Kollision und schließlich zur Koaleszenz führen. Allerdings ließ sich dies nur für den ungestörten Strahlzerfall beobachten. Im Falle des extern gesteuerten Zerfalls in monodisperse Tropfen zeigten sich keine messbaren Geschwindigkeitsschwankungen zwischen benachbarten Tropfen ${ }^{12}$, Mit größerem Abstand zur Düse steigt jedoch auch der Einfluss durch Reibung in der umgebenden Luft und so verkürzen sich die Tropfenabstände soweit, bis es auch in diesem Fall schließlich zur Koaleszenz kommt.

Beispielhafte Verteilungen der Tropfendurchmesser und Zeitreihen der dazugehörenden Hochgeschwindigkeitsaufnahmen sind in Abb. $1.15 \mathrm{zu}$ finden. Für jedes Verteilungsspektrum wurden mehrere tausend Tropfen analysiert. Die Spektren ohne akustische Anregung sind für einen Abstand von (a) $2 \mathrm{~mm}$ und (b) $20 \mathrm{~mm}$ unterhalb der Düse und das Spektrum für den extern gesteuerten Zerfall mit akustischer Anregung für einen Abstand von (c) $15 \mathrm{~mm}$ unterhalb der Düse gezeigt. In Abb. 1.15a ist in der Verteilung eine isolierte Gruppe kleiner Tropfen (Fragmente) zu sehen, die klar von den größeren Tropfen getrennt ist. Solche Satellitentröpfchen werden häufig während des Ablöseprozesses der größeren Tropfen vom Flüssigkeitsstrahl beobachtet, wie es auch in Abb. 1.4a zu sehen ist.

12 Persönliche Bemerkung: Während stroboskopischer Aufnahmen der Tropfenkette kam es wiederholt zu der bestätigenden Frage: „Läuft der Film oder ist es ein Standbild?“. 
Des Weiteren weist die Verteilung ein deutliches Maximum bei einem Durchmesser von etwa $38 \mu \mathrm{m}$ auf. Dies entspricht der am schnellsten wachsenden Mode $k a_{\text {opt }} \approx 0,69$ für die $20 \mu \mathrm{m}$ Düse. Das Minimum der Tropfengrößen bei der begrenzenden Wellenzahl $D_{\min }(k a=1,0) \approx 34 \mu \mathrm{m}$ lässt sich sehr gut nachvollziehen. Es liegt ebenfalls nahe der empirisch gefundenen Begrenzung des akustisch angeregten Strahlzerfalls [79] (ähnlich zu dem Befund in Abb. 1.13) mit $D_{\min }\left(k a_{\max }=0,9\right) \approx 35 \mu \mathrm{m}$. Die empirische Grenze des steuerbaren Zerfalls in große Tropfen von $D_{\max }\left(k a_{\min }=0,3\right) \approx 50 \mu \mathrm{m}$ ist nicht im Histogramm zum ungestörten Strahlzerfall ersichtlich, und es bilden sich teils deutlich größere Filamente, wie auch in Abb. 1.4a zu sehen. Der Wertebereich erstreckt sich bis etwa $80 \mu \mathrm{m}$ und weist Modulationen auf, was, wie schon erwähnt, teilweise auf Kollision mit Nachbartropfen zurückzuführen ist. Je weiter unterhalb der Düse die Messung stattfindet, desto deutlicher wird dieser Effekt, und das Größenspektrum verschiebt sich hin zu noch größeren Werten.

Im Spektrum nach einer Strecke von etwa $20 \mathrm{~mm}$ (siehe Abb. 1.15b) sind keine Satellitentröpfchen mehr vorhanden. Außerdem erscheinen im Spektrum Maxima bei Tropfen mit dem doppelten $\left(2^{*}\right)$, dreifachen $\left(3^{*}\right)$ und vierfachen $\left(4^{*}\right)$ Volumen bezogen auf einen ,optimalen“ Durchmesser von $38 \mu \mathrm{m}$. Zwischen dem globalen Maximum der „optimalen“ Tropfengröße und den Maxima der Vielfachen ist eine klare Abtrennung der beiden Bereiche zu erkennen. Der gesamte Wertebereich reicht in diesem Fall bis zu Tropfengrößen von etwa $100 \mu \mathrm{m}$. Während einige größere (längliche) Tropfen schon kurz unterhalb der Düse auftreten (vergleiche Abb. 1.4a zerfallen sie meist recht schnell in kleinere Fragmente 43, 88.

Abbildung 1.15 (nächste Seite): Größenverteilung der Tropfendurchmesser für den Zerfall des Flüssigkeitsstrahls für die $20 \mu \mathrm{m}$ Edelstahldüse, DI Wasser und eine Geschwindigkeit von etwa $27 \mathrm{~m} \mathrm{~s}^{-1}$. Ungestörter Zerfall: (a) $2 \mathrm{~mm}$ und (b) $20 \mathrm{~mm}$ unterhalb der Düse. Durch nachfolgende Koaleszenz unterhalb der Düse verschwinden die Fragmente und es erscheinen Maxima bei Vielfachen der zuvor dominierenden Tropfengröße $(38 \mu \mathrm{m})$. Die Durchmesser, die dem doppelten, dreifachen und vierfachen Volumen eines $38 \mu \mathrm{m}$ Tropfen entsprechen sind mit $2^{*}(48 \mu \mathrm{m})$, $3^{*}(55 \mu \mathrm{m})$ und $4^{*}(60 \mu \mathrm{m})$ gekennzeichnet. Im Vergleich dazu weist das Spektrum $15 \mathrm{~mm}$ unterhalb der Düse (c) bei aktiver akustischer Anregung mit $300 \mathrm{kHz}$ ein scharf ausgeprägtes Maximum bei einem einzelnem Durchmesser in Abwesenheit weiterer Tropfengrößen auf. Neben den Größenverteilungen sind jeweils Zeitreihen der Hochgeschwindigkeitsaufnahmen gezeigt, welche zu je aus 20 aufeinanderfolgenden Bildern bestehen. Die Bildrate betrug $1 \mathrm{kfps}$ in (a) und (b) und $100 \mathrm{kfps}$ in (c), immer mit einer Belichtungszeit von 369 ns. Die Daten wurden jeweils über einen Zeitraum von $0,1 \mathrm{~s}$ ermittelt. 


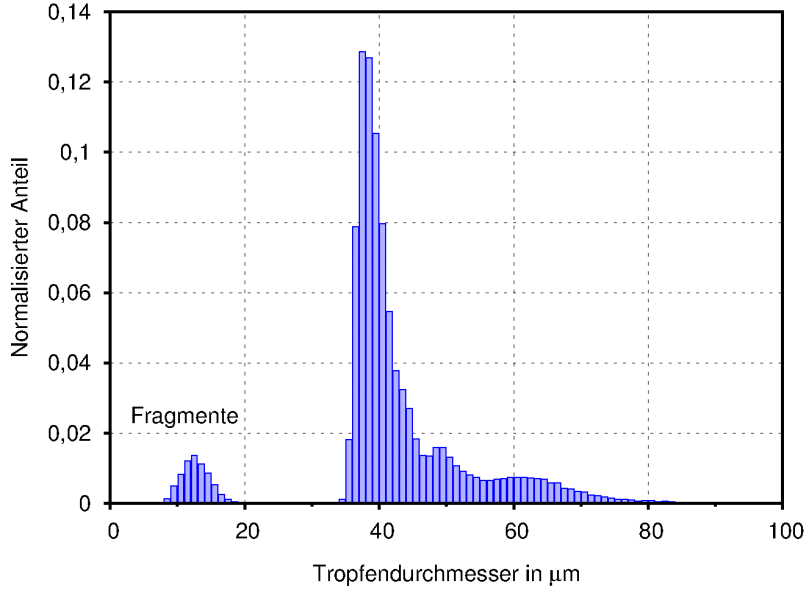

(a)

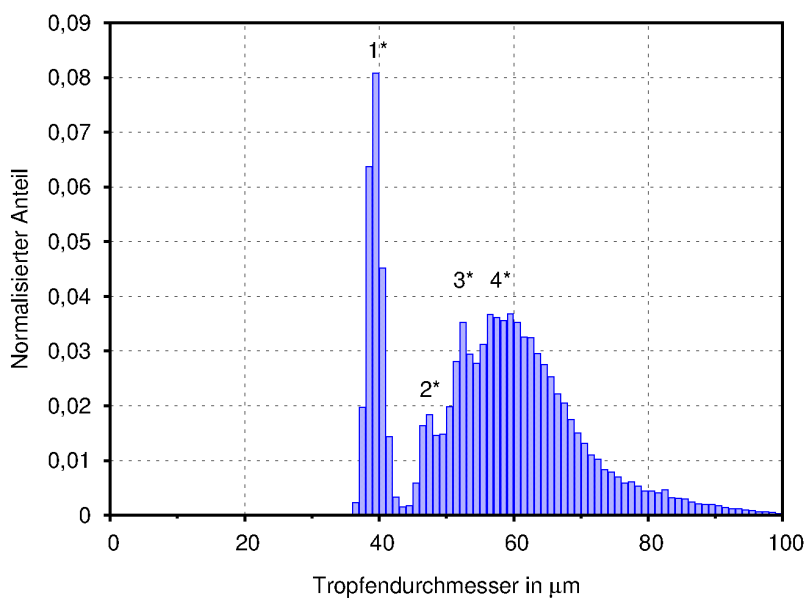

(b)

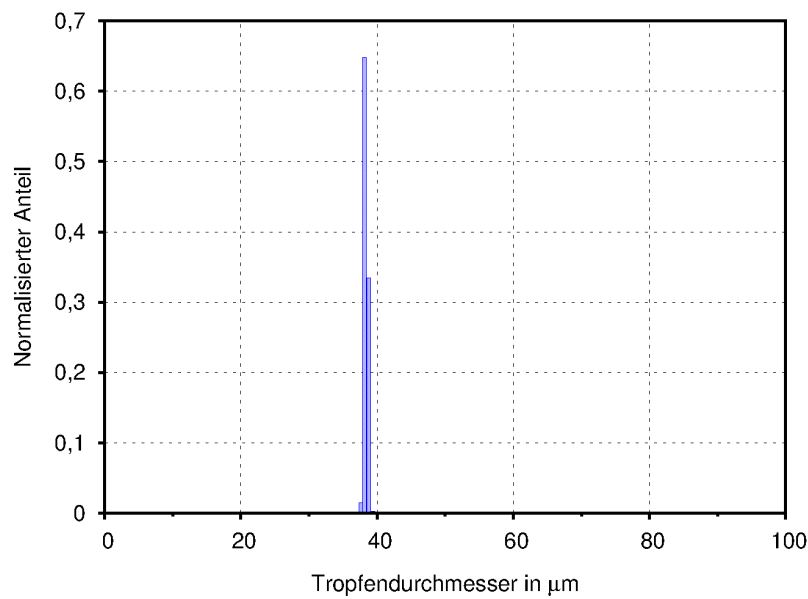

(c)
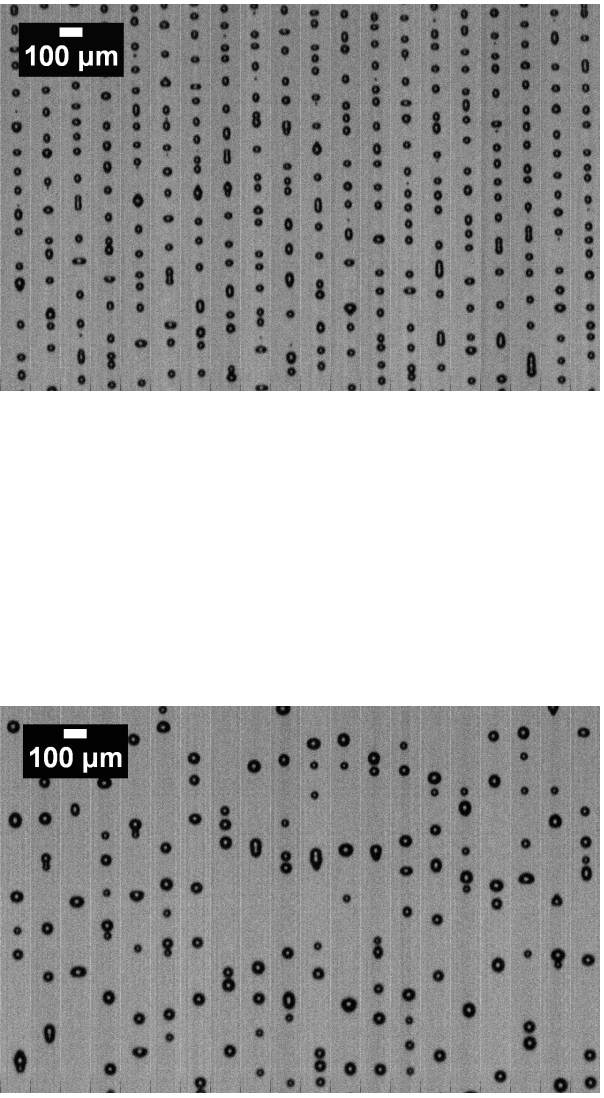

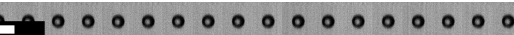

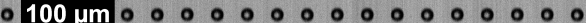

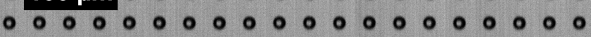

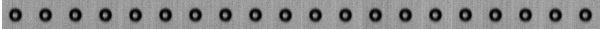

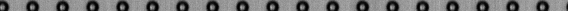

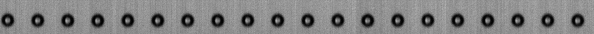




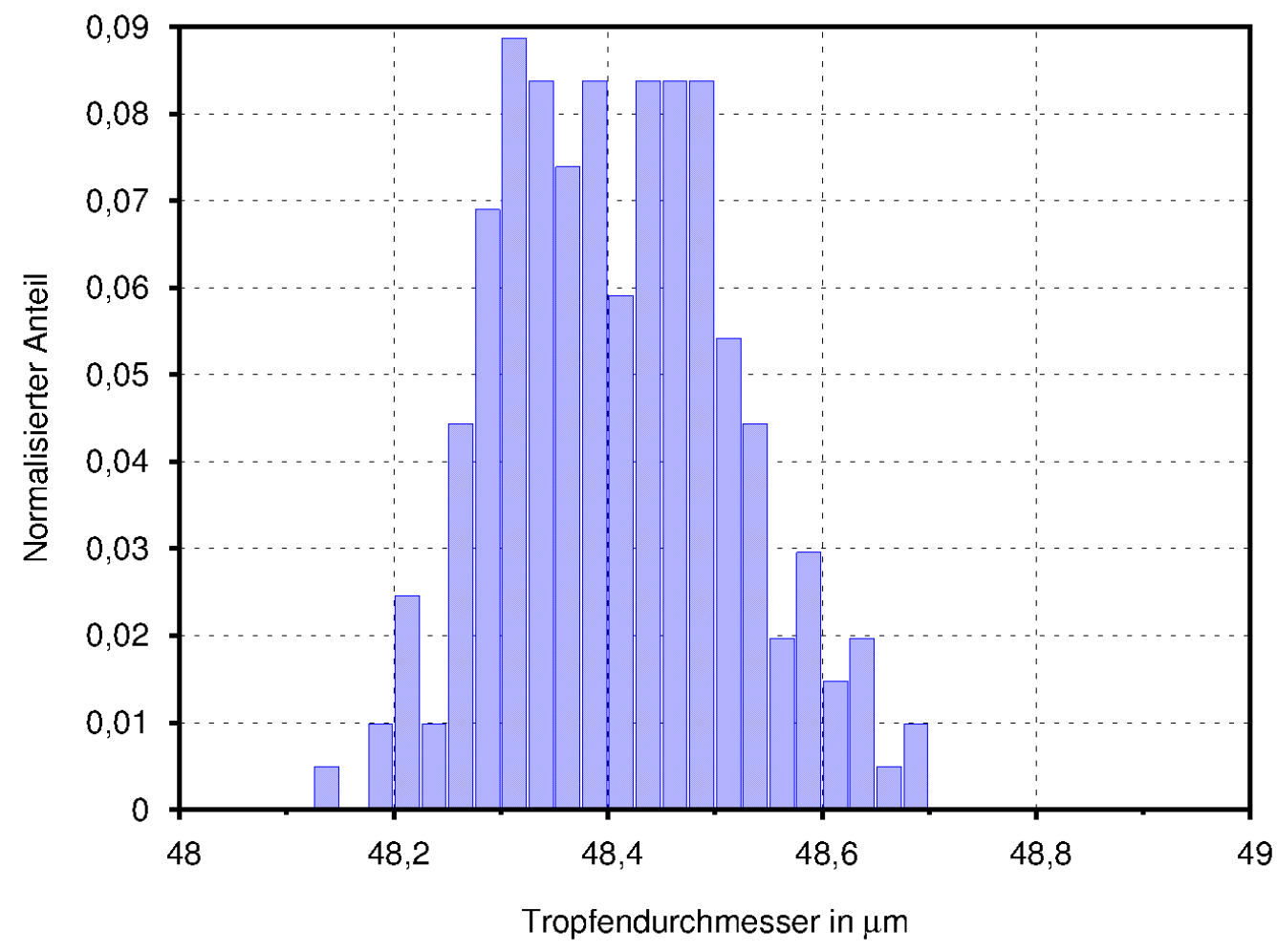

Abbildung 1.16: Größenverteilung der Tropfen unter Verwendung einer synchronisierten, stroboskopischen Aufnahme zur genaueren Bestimmung der Durchmesser.

Daher ist das Vorkommen größerer Tropfen in der Verteilung $20 \mathrm{~mm}$ unterhalb der Düse bereits durch Kollision/Koaleszenz und nicht durch Details des Zerfallsprozesses des Flüssigkeitsstrahls bestimmt.

Wird jedoch die akustische Anregung mit einer passenden Frequenz benutzt, so wird die Tropfenkette zu einem hohen Grade monodispers, wie in Abb. $1.15 \mathrm{c} z u$ sehen. Es zeigte sich, dass die beobachtete Breite der sehr schmalen Verteilung $(\approx \pm 1 \mu \mathrm{m}$ bzw. $3 \%)$ tatsächlich nicht der realen Schwankungsbreite entspricht, sondern ein Artefakt der Messmethode ${ }^{13}$ ist und in der Relaxationsoszillation der Tropfenform beruht $\left[89 \mid\right.$. In einem ausgewählten Fall für $U=20 \mathrm{~m} \mathrm{~s}^{-1}$ wurde die Monodispersität mit einer höheren Vergrößerung (Auflösung $\approx 0,7 \mu \mathrm{m} / \mathrm{pix}$ ) und synchronisierter, stroboskopischer Beleuchtung (Belichtungszeit 7 ns, entspricht

13 Da die Hochgeschwindigkeitsaufnahmen nicht mit der Tropfenerzeugung/-frequenz synchronisiert waren, variierte die Tropfenposition und damit die Phase der Relaxationsoszillation um die sphärische Grundform für jeden gemessenen Tropfen. Obwohl die ellipsoide Form auf ein achsensymmetrisches Volumen korrigiert wurde, führten die Begrenzung durch die optische Auflösung ( $\approx 2 \mu \mathrm{m} / \mathrm{pix})$ und die Bewegungsunschärfe zu der beobachteten Variation in der Tropfengröße. 


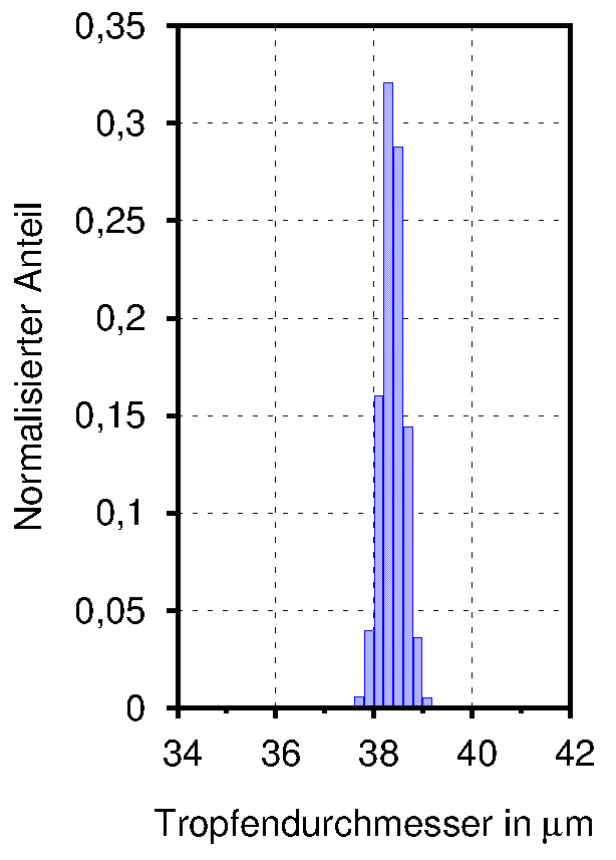

(a)

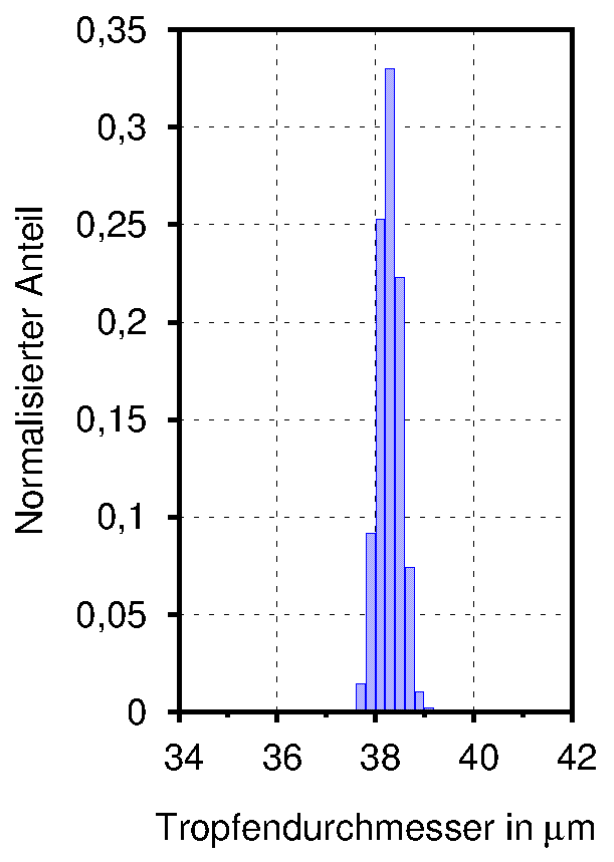

(b)

Abbildung 1.17: Vergleich der Verteilungen der Tropfendurchmesser für dieselben Parameter wie in Abb. 1.15, jedoch über verschiedene Zeitbereiche ermittelt: (a) $0,1 \mathrm{~s}$ und (b) $5 \mathrm{~s}$. Es ist jeweils das deutliche Maximum bei etwa $38,2 \mu \mathrm{m}$ zu erkennen. Auch für den größeren Zeitbereich (b) bleibt das Spektrum nahezu unverändert.

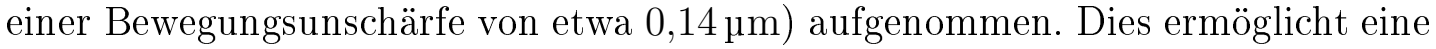
genauere Untersuchung der Tropfenkontur/-größe (siehe hierfür Abb. 1.16). Die Analyse hiervon ergab eine Standardabweichung von etwa $0,4 \%$, wobei insbesondere die größten und kleinsten vorkommenden Tropfendurchmesser nicht mehr als $1 \%(\sim \pm 0,4 \mu \mathrm{m})$ vom Mittelwert abwichen. Zusammenfassend lässt sich festhalten, dass die tatsächliche Güte der Monodispersität innerhalb der experimentell zugänglichen Grenzen liegt und damit als hinreichend exakt angenommen werden kann.

Um die Langzeitstabilität der Methode zu überprüfen, wurde im Vergleich zu den vorherigen Aufnahmen/Daten (Dauer etwa 0,1s) das Spektrum außerdem über einen Zeitraum ${ }^{14}$ von $5 \mathrm{~s}$ analysiert (siehe Abb. 1.17). Die Monodispersität ist auch über Sekunden hinweg gegeben und es ist davon auszugehen, dass dies auch über noch längere Zeiträume gültig ist, solange alle Kontrollparameter (Druck, Frequenz, Spannung, etc.) absolut konstant gehalten werden.

14 Die Dauer der Aufnahme war durch den internen Speicher der Kamera beschränkt. 


\subsubsection{Vergleich unterschiedlicher Düsendurchmesser}

Der zuvor hergeleitete Zusammenhang zwischen der Tropengeschwindigkeit $U$ und dem Druck $\Delta p$ soll für die weiteren verwendeten Düsendurchmesser überprüft werden.

In Abb. 1.18 sind für vier exemplarische Siliziumdüsen (mit Durchmessern: $3,9 \mu \mathrm{m}, 8,2 \mu \mathrm{m}, 16,8 \mu \mathrm{m}$ und 25,2 $\mu \mathrm{m})$ die Messdaten und die jeweils entsprechenden Fitparameter $C_{1}$ und $C_{2}$ aufgeführt. Alle Düsen lassen sich gemäß Gl. (1.2) beschreiben, ergeben jedoch (obwohl sie bis auf den Düsendurchmesser von der Düsengeometrie her identisch sind) jeweils individuell unterschiedliche Fitparameter. Für alle Düsendurchmesser wurde wie schon in Kap 1.4.1 1.4.4 ebenfalls die Funktionalität der Erzeugung monodisperser Tropfen untersucht und für ebenso gültig befunden. Es ist ebenfalls zu sehen, dass sich ein kleiner werdender Düsendurchmesser in einem höheren notwendigen Druck zum Erzielen derselben

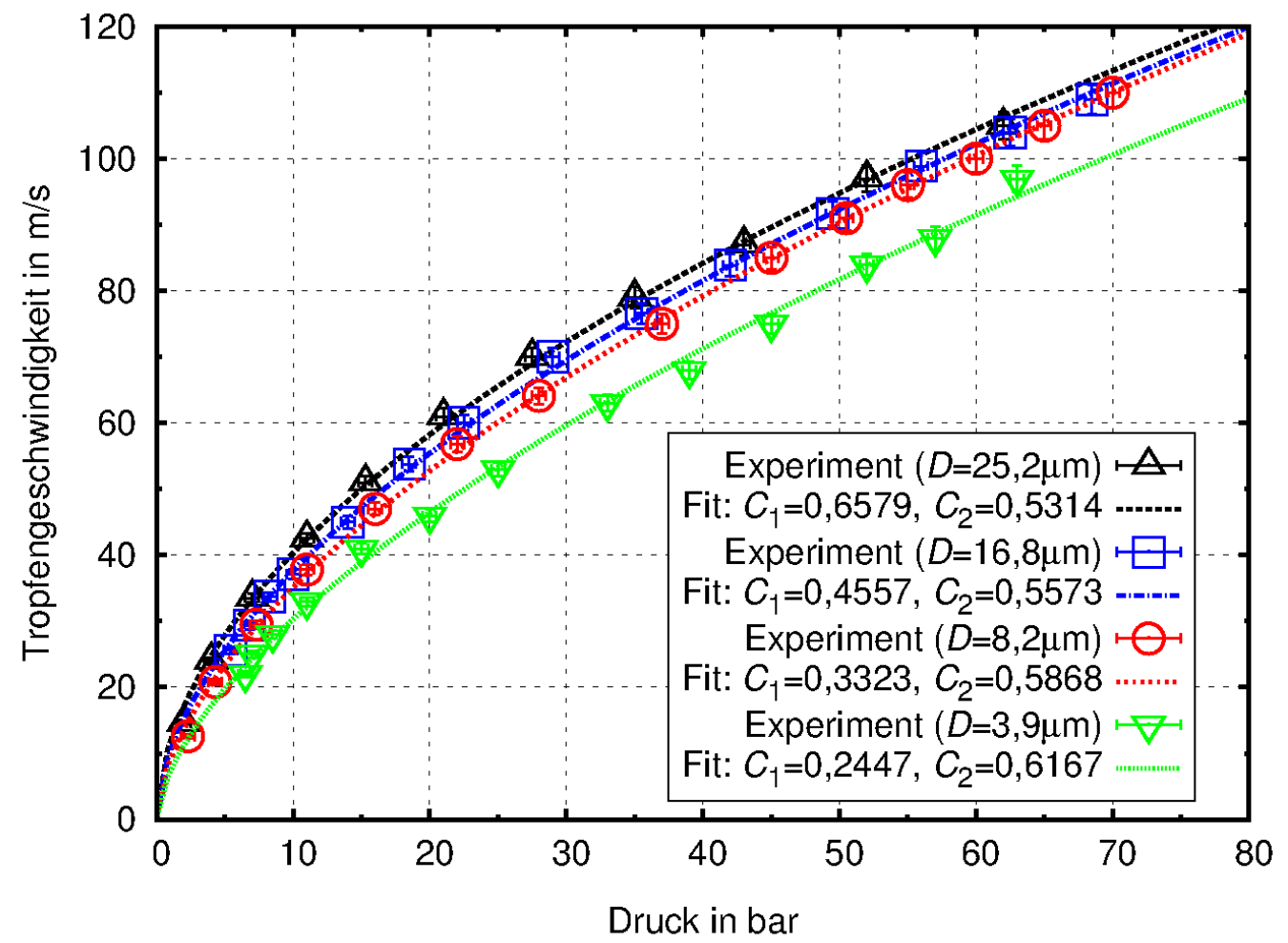

Abbildung 1.18: Vergleich verschiedener Siliziumdüsen bzgl. der minimal und maximal erreichbaren Tropfengeschwindigkeiten beim gesteuerten Zerfall des Flüssigkeitsstrahls in monodisperse Tropfen durch akustische Anregung. Es ist möglich, jede Düse mittels Gl. 1.2 zu beschreiben, wobei sich unterschiedliche Fitparameter $C_{1}$ und $C_{2}$ ergeben. 
Geschwindigkeit äußert. Der Einfluss der sich ändernden Düsendurchströmung äußert sich nicht zuletzt auch in sinkenden Parametern $C_{1}$ und steigenden Parametern $C_{2}$ für kleiner werdende Düsendurchmesser.

Da es bei festem Düsendurchmesser möglich ist, die Tropfengröße um $-9 \%$ und $+32 \%$ bezogen auf $D_{\text {opt }}\left(k a_{\text {opt }}=0,69\right)$ zu variieren (siehe Kap. 1.4.3), scheint es zunächst möglich, die sich daraus ergebenden Überlappungen der Tropfendurchmesser für eine Druckreduzierung zu nutzen. In den meisten Fällen ergibt sich, selbst bei Annahme einer zu erzielenden Tropfengeschwindigkeit von $100 \mathrm{~m} \mathrm{~s}^{-1}$, dabei jedoch lediglich eine Reduktion von 1 bar oder weniger. 


\subsection{Zusammenfassung}

Ausgehend von den Ergebnissen in [40 und [79 wurden Mikrobohrungen der Größenordnung $20 \mu \mathrm{m}$ als Düsen verwendet. Ein angelegter Überdruck führt zum Austreten eines Flüssigkeitsstrahls in die umgebende Luft durch die Düse, welche mit einem Flüssigkeitsreservoir verbunden ist. Der Zerfall dieses Strahls wird durch eine externe Störung im Ultraschallbereich überlagert, welche durch mechanische Vibrationen eines piezokeramischen Schallwandlers erzeugt wird. Innerhalb eines gewissen Frequenzbereiches, der von den jeweiligen Strahlparametern (wie z.B. Durchmesser, Geschwindigkeit, Flüssigkeitsparameter, etc.) abhängig ist, lässt sich der Zerfall derart kontrollieren, dass sich exakt ein Tropfen pro akustischer Periode vom Strahl löst. Als Ergebnis entsteht eine Tropfenkette mit einer einheitlichen Tropfengröße und gleichzeitig auch einheitlicher Tropfengeschwindigkeit.

Die Auswertungen konnten sowohl die Funktionsweise der Methode als auch ihre Güte im Bezug auf die Erzeugung eines Tropfensprays mit kontrollierbarer und variabler Tropfengröße und Tropfengeschwindigkeit unter Beweis stellen. Es wurden Tropfendurchmesser im Bereich $D \approx 7 \mu \mathrm{m}$ bis $65 \mu \mathrm{m}$, Tropfengeschwindigkeiten im Bereich $U \approx 10 \mathrm{~m} \mathrm{~s}^{-1}$ bis $120 \mathrm{~m} \mathrm{~s}^{-1}$ und Erzeugungsraten im Bereich von mehreren hunderttausend Tropfen pro Sekunde erzielt.

Für Geschwindigkeiten bis zu $\lesssim 38 \mathrm{~m} \mathrm{~s}^{-1}$ wurde der Zerfall in monodisperse Tropfen für die Flüssigkeiten DI Wasser, 2-Propanol, Aceton, Ethylenglykol, 1-Hexanol und 1-Butanol untersucht. Für alle betrachteten Flüssigkeiten konnte die Methode erfolgreich angewendet werden. Die in $|79|$ verwendete Vorgehensweise zur Beschreibung des Zusammenhangs zwischen angelegtem Überdruck und resultierender (Tropfen- und) Strahlgeschwindigkeit mittels zweier Fitparameter $C_{1}$ und $C_{2}$ ließ sich für alle Flüssigkeiten und eine $20 \mu \mathrm{m}$ Düse bestätigen. Jedoch ließ sich die ebenfalls vermutete Vereinheitlichung zu allgemeinen Parametern $C_{1}$ und $C_{2}$ für alle Flüssigkeiten nicht konsistent nachvollziehen. Insbesondere für Flüssigkeiten mit höherer Viskosität (wie z.B. Ethylenglykol) ergaben sich Abweichungen von bis zu 30\%. Ein Zusammenhang von $C_{1}$ und $C_{2}$ mit der Ohnesorge-Zahl scheint wahrscheinlich, jedoch sind hierfür weitergehende Experimente notwendig. Es lässt sich festhalten, dass für jede Düsengeometrie und Flüssigkeit $C_{1}$ und $C_{2}$ individuell bestimmt werden müssen, um hinreichend genaue Anpassungen zu erreichen. Diese lassen sie sich jedoch anschließend sehr gut und genau zur Interpolation über den gesamten Wertebereich der Strahlgeschwindigkeit als Funktion des Druckes benutzen. Die Abweichungen von der angepassten Kurve liegen dann typischerweise im einstelligen Prozentbereich, häufig auch darunter. 
Im Weiteren wurde untersucht, welcher Geschwindigkeitsbereich sich mit der Methode abdecken lässt. Die untere Grenze ist hauptsächlich durch die Bedingung gegeben [83], dass die kinetische Energie des Flüssigkeitsstrahls die Oberflächenspannung an der Düsenöffnung überschreiten muss. Dies konnte annähernd nachvollzogen werden, war aber nicht von weiterem Interesse. Die obere Grenze, welche durch den Übergang zur als „Zerwellen“ bezeichneten Instabilität 84 gegeben ist, wurde um mehr als das Doppelte überschritten. Dabei wies der Flüssigkeitsstrahl keine dementsprechende Störung auf und gleichzeitig ließ sich der Zerfall in monodisperse Tropfen bis zu einer Geschwindigkeit von etwa $120 \mathrm{~m} \mathrm{~s}^{-1}$ erreichen. Die räumliche Nähe zu weiteren Flüssigkeitsstrahlen zeigte jedoch einen deutlichen Einfluss auf die Strahlform, welche eine Wellenbewegung quer zur Ausbreitungsrichtung entwickelte. Dennoch ließ sich der Strahlzerfall in monodisperse Tropfen kontrollieren. Es bleibt an dieser Stelle offen, was die maximal möglichen Tropfengeschwindigkeiten mit dieser Methode und den verwendeten Düsen sind, da einer weiteren Erhöhung des Druckes experimentelle Beschränkungen gegenüberstanden. Insbesondere bleibt zu klären, ob der extern getriebene Zerfall sich auch im Zerwellen-Regime fortsetzten lässt bzw. ob damit der Übergang zu dieser Instabilität hinausgezögert werden kann.

Innerhalb eines gewissen Bereiches lassen sich bei festem Düsendurchmesser die erzeugten Tropfendurchmesser verändern, typischerweise $-9 \%$ und $+32 \%$ bezogen auf $D_{\text {opt }}\left(k a_{\text {opt }}=0,69\right)$. Für den gesamten Geschwindigkeitsbereich konnten die erwarteten Änderungen des Frequenzbereiches validiert werden. Es zeigte sich, dass bei hinreichend starker akustischer Anregung innerhalb von $f_{\min }$ und $f_{\max }$ durchgehend ein kontrollierter Strahlzerfall in monodisperse Tropfen gegeben ist.

Die Position des Strahlzerfalls in Tropfen, sprich die Zerfallslänge, lässt sich mit aktiver akustischer Kontrolle deutlich verkürzen, wie auch in [43] mit einer Amplitudenabhängigkeit der Störung beschrieben. Bis zu einer Geschwindigkeit von $90 \mathrm{~m} \mathrm{~s}^{-1}$ ließen sich die Tropfen innerhalb der ersten $3 \mathrm{~mm}$ unterhalb der Düse erzeugen, was in etwa einem Drittel der Zerfallslänge (bzw. Zerfallszeit) im ungestörten Zerfall entspricht. Da die Zerfallslänge direkt mit der Amplitude der Störung zusammenhängt, ist zu erwarten, dass sie sich durch höhere Spannungen am piezokeramischen Schallwandler bzw. eine höhere mechanische Anregung noch weiter reduzieren ließe. Für eine mögliche praktische Anwendung bedeutet dies, dass der Arbeitsabstand größer als diese Länge, aber gleichzeitig kleiner als die Koaleszenzlänge (d.h. die Position wo die individuellen Tropfen aufgrund der Abbremsung der Tropfenkette miteinander kollidieren) sein sollte. Als Richtwert erwies sich in faktisch allen Fällen ein Bereich zwischen $5 \mathrm{~mm}$ und $15 \mathrm{~mm}$ als zuverlässig. 
Die Untersuchung der Verteilung der Tropfengrößen für den freien Zerfall des Strahls zeigte ein Anwachsen der Tropfengröße mit steigendem Abstand von der Düse durch Kollision von Nachbartropfen. Im Gegensatz dazu erwies sich der im passenden Frequenzbereich akustisch angeregte Zerfall als sehr präzise monodispers. In allen Experimenten stellte sich die optische Auflösung der Hochgeschwindigkeitsaufnahmen als die hauptsächliche Ursache für beobachtete Schwankungen der Tropfengröße heraus. In ausgewählten Experimenten mit höherer Vergrößerung und kürzerer Belichtungszeit ließ sich eine Standardabweichung von weniger als $0,5 \%$ bestimmen. Diese hohe Genauigkeit erwies sich auch über den Bereich mehrerer Sekunden als stabil. Eine Langzeitstabilität ist daher zu erwarten und wäre weniger durch den Zerfallsprozess an sich als vielmehr durch Schwankung anderer Prozessparameter wie z.B. Überdruck oder Anregungsamplitude limitiert.

Zusammenfassend lassen sich folgende Aussagen treffen. Ausgehend vom breiten Parameterbereich der Experimente konnten Funktionalität und Qualität der Methode unter Beweis gestellt werden. Ebenso wurden ihre Grenzen im Rahmen der erreichbaren Geschwindigkeiten untersucht. Insbesondere konnte gezeigt werden, dass der Zerfall des Flüssigkeitsstrahls in monodisperse Tropfen auch jenseits der in 84 beschriebenen Schranken möglich ist. Für den gesamten Wertebereich ließ sich der theoretische Ansatz von [79] verwenden, womit sich mittels zweier (zu bestimmender) Parameter der Zusammenhang zwischen Überdruck und Tropfengeschwindigkeit mit hoher Genauigkeit beschreiben lässt. Mit Hinblick auf die Anforderungen eines Reinigungsprozesses von sensiblen, strukturierten Oberflächen wird ersichtlich, dass durch die definierte Tropfengröße eine genaue Festlegung der involvierten Einschlagenergien und -impulse der Tropfen möglich ist. Es ist zu erwarten, dass mit (noch etwas) kleineren und größeren Düsen der gesamte Bereich von $D \approx 10 \mu \mathrm{m}$ etwa $100 \mu \mathrm{m}$ und $U \approx 10 \mathrm{~m} \mathrm{~s}^{-1}$ bis $100 \mathrm{~m} \mathrm{~s}^{-1}$ gezielt mit einer bestimmten Kombination aus $D$ und $U$ erreichbar ist (siehe auch [90 und 91]). Dadurch erfüllt diese Methode mit hoher Präzision die Anforderungen solcher Anwendungen, da sich so die vermutlich schmalen Übergänge zwischen Reinigung und Erosion identifizieren lassen. Abschließend ist zur Veranschaulichung in Abb. 1.19 der in der vorliegenden Untersuchung abgedeckte Bereich dem durch eine exemplarische Anwendung vorgegebenen Parameterraum gegenübergestellt. 


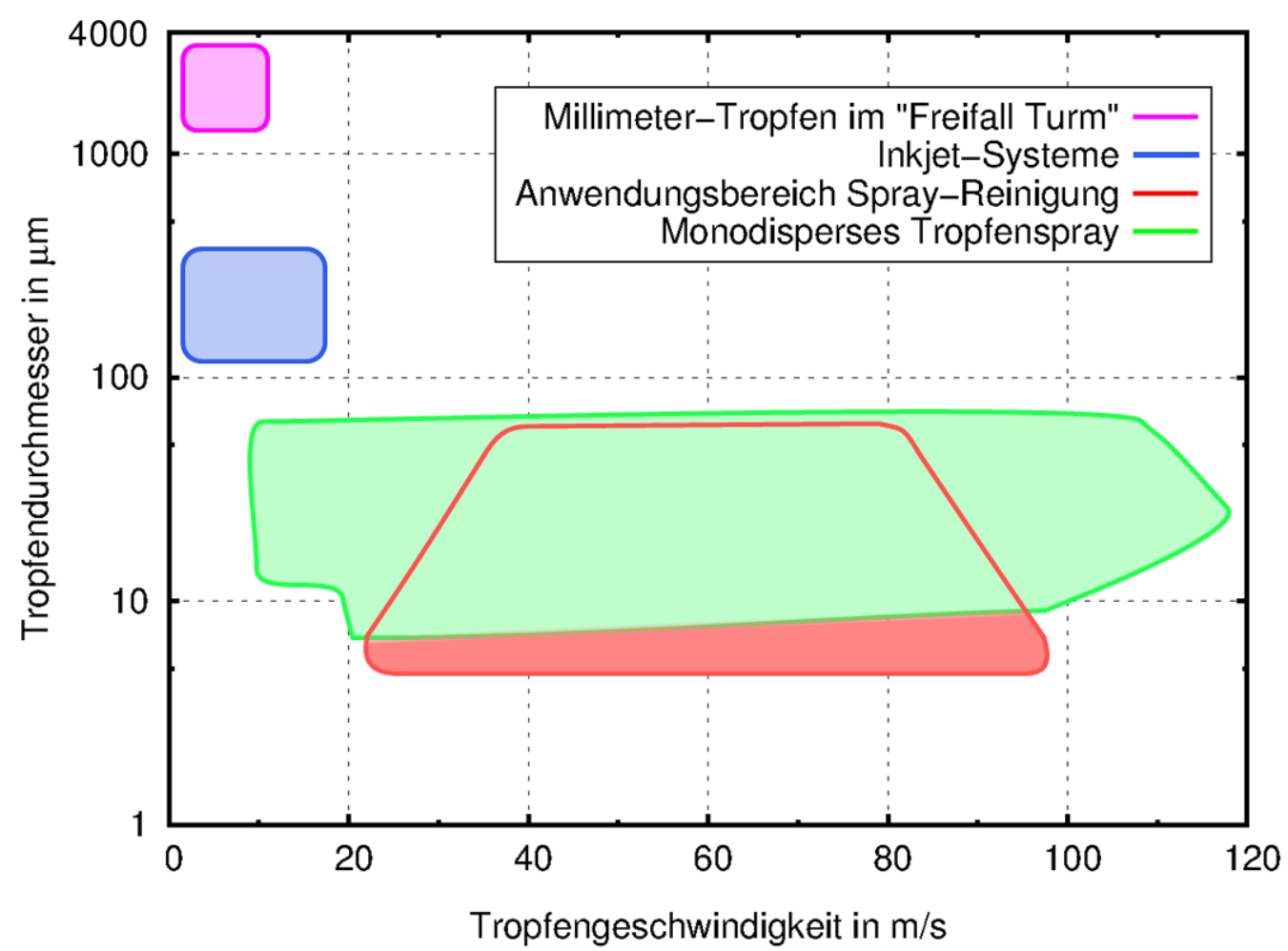

Abbildung 1.19: Vergleich der Anforderungen typischer Techniken der Spray-Reinigung (rot) mit drei Ansätzen für monodisperse Tropfenquellen. Tropfen, die mittels Kapillaren erzeugt und durch die Schwerkraft beschleunigt werden, sind typischerweise im Größenbereich von wenigen Millimetern und überschreiten in Luft häufig nicht Geschwindigkeiten von $10 \mathrm{~m} \mathrm{~s}^{-1}$ (violett). Aus dem Bereich der Drucktechnik (Inkjet- oder DOD-Systeme) besteht die Möglichkeit, Tropfendurchmesser von etwa einer Größenordnung kleiner zu erzeugen (blau). Jedoch weist die Tropfenform insbesondere bei Geschwindigkeiten $\gtrsim 10 \mathrm{~m} \mathrm{~s}^{-1}$ häufig eine Asphärizität auf, und es kann zur Bildung nachfolgender (unerwünschter) Satellitentropfen kommen. Das in der vorliegenden Arbeit behandelte monodisperse Tropfenspray (im Bezug auf Tropfengröße und -geschwindigkeit) lässt eine gezielte Erzeugung über einen weiten Parameterbereich zu (in dieser Arbeit erreichte Parameter: grün). Die Funktionalität konnte für $D \approx 7 \mu \mathrm{m}$ bis $65 \mu \mathrm{m}$ und $U \approx 10 \mathrm{~m} \mathrm{~s}^{-1}$ bis $120 \mathrm{~m} \mathrm{~s}^{-1}$ gezeigt werden, was einen Großteil der Anforderung der Spray-Reinigung abdeckt. Der verbleibende (bis dato noch nicht untersuchte) Bereich für noch kleinere Tropfen ist aller Voraussicht nach mit noch kleineren Düsen und der gezeigten Methode monodispers erreichbar. 


\section{Kapitel 2}

\section{Erzeugung und Untersuchung des Aufpralls einzelner Mikrotropfen*}

\subsection{Motivation}

Eine Vielzahl experimenteller Untersuchungen in jüngerer Vergangenheit (z.B. 9, 19, 23, 28, 92, 93 ) behandelt den Aufprall von Tropfen mit einem Durchmesser $D \gtrsim 1 \mathrm{~mm}$ bei geringen Geschwindigkeiten $U_{0} \lesssim 10 \mathrm{~m} \mathrm{~s}^{-1}$, während nur vergleichsweise wenige Arbeiten zum Einschlag von Tropfen mit einem Durchmesser $D \lesssim 100 \mu \mathrm{m}$ bei hohen Geschwindigkeiten $U_{0} \gtrsim 10 \mathrm{~m} \mathrm{~s}^{-1}$ existieren (z.B. 70,91]). Grund hierfür ist die Begrenzung in der Aufnahmetechnik, einen einzelnen Tropfeneinschlag sowohl räumlich als auch zeitlich aufzulösen. Die typische Zeitskala lässt sich aus dem Verhältnis der Einschlagsgeschwindigkeit zum Tropfendurchmesser abschätzen, z.B.

$$
\tau=\frac{U_{0}}{D}=\frac{50 \mathrm{~m} \mathrm{~s}^{-1}}{50 \mu \mathrm{m}}=1 \mu \mathrm{s}
$$

Selbst modernste Hochgeschwindigkeitskameras [94 erreichen bei ausreichender optischer Auflösung selten mehr als $1 \mathrm{Mfps}$, was ungefähr der Zeitskala für den Tropfeneinschlag entspricht. Da Tropfen aus diesem Parameterbereich jedoch Bestandteil vieler aktueller Anwendungen sind, ist es notwendig, die beim Aufprall involvierten Prozesse zu verstehen.

Der Inhalt dieses Kapitels gliedert sich in zwei Teile. Im ersten Teil wird die Erzeugung eines einzelnen Mikrotropfens auf Grundlage der in Kap. 1 beschriebenen Methode zu monodispersen Tropfensprays erläutert. Mit Hilfe zeitlich präziser Hochspannungspulse wird ein einzelner Tropfen elektrisch aufgeladen und abgelenkt, um auf einem Substrat einzuschlagen. Dabei gilt als Maßgabe, 
die hohe Reproduzierbarkeit aus Kap. 11 auf den Einzeltropfen zu übertragen. Außerdem werden die Beschränkungen der Methode und die maximal erreichbaren Einschlagsgeschwindigkeiten für Tropfendurchmesser von $10 \mu \mathrm{m}$ bis $100 \mu \mathrm{m}$ abgeleitet. Im zweiten Teil wird der Aufprall eines einzelnen Mikrotropfens behandelt. Die Reproduzierbarkeit des Tropfeneinschlags ermöglicht es, mittels repetierter Aufnahmen mit Zeitversatz (stroboskopische Aufnahmen) den Tropfeneinschlag in zeitlich hoher Auflösung von bis zu $10 \mathrm{~ns}$ aufzunehmen, was äquivalent zu einer Aufnahmegeschwindigkeit von $100 \mathrm{Mfps}$ ist. Die Aufnahme erfolgt zeitlich synchronisiert von der Seite und von unten. Es wird diskutiert, inwieweit validierte Modelle zu langsamen, millimetergroßen Tropfen auch für schnelle, mikrometergroße Tropfen Gültigkeit besitzen.

Die Beschreibung der verwendeten experimentellen Methoden ist in Kap. 2.2 gegeben. Der theoretische Hintergrund und die erzielten Resultate werden zur Erzeugung einzelner Mikrotropfen in Kap. 2.3 und zum Aufprall einzelner Mikrotropfen in Kap. 2.4 dargestellt und diskutiert. Eine Zusammenfassung der Ergebnisse folgt in Kap. 2.5.

\footnotetext{
${ }^{*}$ Die in diesem Kapitel dargestellten experimentellen Methoden und Ergebnisse sind aus der Kooperation mit C. W. Visser (Physics of Fluids, University Twente, Holland) entstanden. Die Entwicklung der Versuchsanordnung in Kap. 2.2 und die Beschreibung der Tropfenaufladung, der Tropfenablenkung und die Bestimmung der Tropfentrajektorie in Kap. 2.3 beruhen zum überwiegenden Teil auf der Arbeit des Autors. Die auf dieser Grundlage durchgeführten Experimente ermöglichten die Untersuchung des Aufpralls einzelner, schneller Mikrotropfen. In Kap. 2.4 werden Abschnitte aus gemeinsamen diesbezüglichen Veröffentlichungen aufgeführt: Visser, Frommhold et al. „Dynamics of high-speed micro-drop impact: numerical simulations

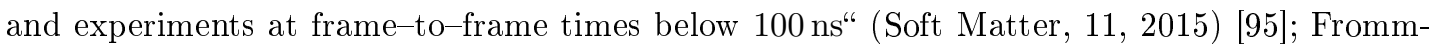
hold, Visser et al. „Generation of precisely controlled high-speed micro droplets" (Konferenzbeitrag, Flow14, Twente, Niederlande, 2014) [96]; Visser, Frommhold et al. „Microdroplet impact“ (Konferenzbeitrag, Focus on Microscopy, Sydney, Australien, 2014) [97.

Ausgehend von der in dieser Arbeit entwickelten Versuchsanordnung wurden Ergebnisse aus der Kooperation mit C.-D. Ohl (School of Physical and Mathematical Sciences, Nanyang Technological University, Singapur) zum Einschlag einer begrenzten Anzahl von Tropfen auf ein tiefe Flüssigkeit und dem daraus resultierenden Lufteinschluss veröffentlicht als: Ohl, Frommhold et al. „Water Entry by a Train of Droplets“ (Konferenzbeitrag, American Physical Society, San Francisco, Kalifornien, 2014) [98].
} 


\subsection{Experimenteller Aufbau}

Abbildung 2.1 zeigt einen schematischen Überblick vom Versuchsaufbau. Ein Flüssigkeitsstrahl zerfällt unter externer akustischer Anregung in monodisperse Tropfen (vgl. Abb. 1.3). Im Moment der Ablösung eines Tropfens vom Strahl wird durch einen kurzzeitigen Hochspannungspuls ein einzelner Tropfen elektrisch aufgeladen. Anschließend lässt sich dieser Tropfen durch ein zeitlich konstantes Hochspannungsfeld aus der (ansonsten neutralen) Tropfenkette herauslenken und kann auf einem (transparenten) Substrat einschlagen, während die verbleibenden Tropfen in einen Auffangbehälter fallen. Zwei synchronisierte Nd:YAG Laser mit iLIF (,illumination by Laser-Induced Fluorescence“, [99]) erzeugen jeweils Doppel-Blitze von wenigen Nanosekunden Dauer, die als Beleuchtungsquelle genutzt werden, um mit zwei Doppelbild-Kameras und Mikroskopobjektiven den Tropfenaufprall sowohl von der Seite als auch von unten aufzunehmen.

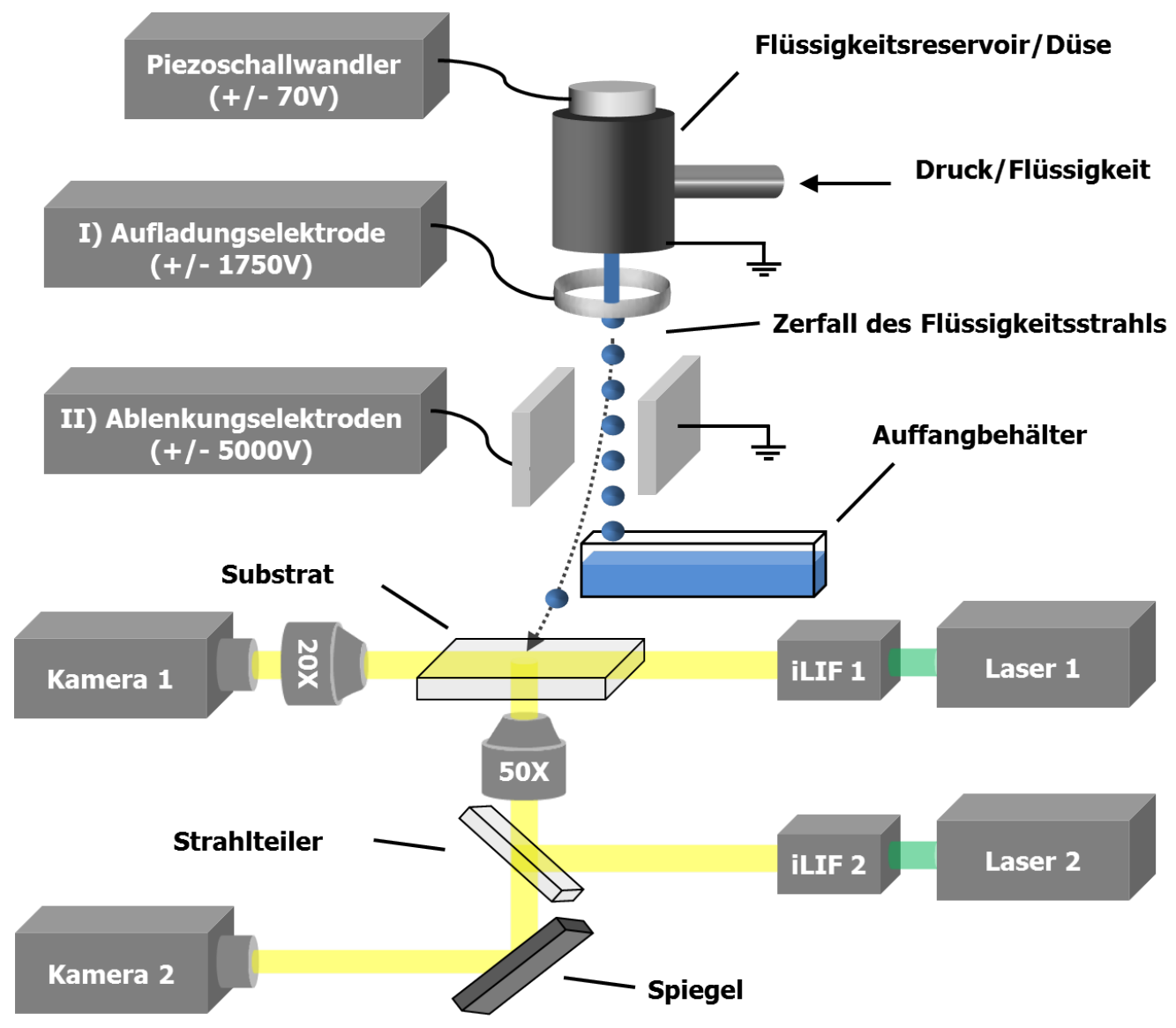

Abbildung 2.1: Schematische Beschreibung der Erzeugung einzelner Einschläge von Mikrotropfen (nicht maßstabsgetreu). 


\subsubsection{Aufladung und Ablenkung von Einzeltropfen}

Die präzise Erzeugung monodisperser Tropfen aus Kap. 1 weist Abweichungen in der Tropfengröße und -geschwindigkeit deutlich unter $1 \%$ auf und dient als Grundlage für das weitere Vorgehen. Die so erzeugte Kette von identischen Tropfen durchläuft einen zweistufigen elektrischen Prozess zur reproduzierbaren Aufladung und Ablenkung eines Einzeltropfens (siehe Abb. 2.2a und 2.2b).

Die erste Instanz wird als „I) Aufladungselektrode“ bezeichnet und ist eine ringförmige Elektrode, welche sich unterhalb der Düse und nahe am Ort der Ablösung der Tropfen vom Flüssigkeitsstrahl befindet, um eine hohe elektrische Feldstärke zu erzeugen. Die Verwendung einer Iris mit variabler Öffnung ermöglicht dabei eine nahe Ausrichtung zum Strahl, ohne ihn zu berühren. Durch Anlegen eines Spannungspulses von bis zu $\pm 1,75 \mathrm{kV}$ (siehe Abb. 2.3) für die Dauer der Tropfenablösung (abhängig von der verwendeten akustischen Anregungsfrequenz) ist es möglich, einen einzelnen Tropfen aufzuladen. Dabei wird die Spitze des Flüssigkeitsstrahls, welcher über die Düse mit dem Schutzleiter verbunden ist, durch eine Verschiebung der Ladungen polarisiert [33]. Beim Ablösen des Tropfens verbleibt somit eine entgegengesetzte Nettoladung auf dem Tropfen. Hierfür ist ein Minimum an verfügbaren Ladungsträgern in der Flüssigkeit notwendig. Während der Aufladungsprozess selbst unter Verwendung von DI Wasser ${ }^{1}$ möglich war, erwies sich das Hinzugeben einer geringen Menge Ammoniak als hilfreich 2 . Prinzipiell ist auch der umgekehrte Prozess, nämlich Aufladen der Tropfenkette und Nichtaufladen des Einzeltropfens, möglich. Im Experiment erwies sich dies jedoch als weit weniger gut kontrollierbar in Bezug auf eine konstante und exakt reproduzierbare Aufladung. Wie später in Kap. 2.4.2 gezeigt wird, ist kein messbarer Einfluss einer Nettoladung oder der im Verhältnis zur Einschlagsgeschwindigkeit kleinen orthogonalen Geschwindigkeitskomponente auf das Einschlagverhalten zu beobachten.

Die zweite Instanz wird als „II) Ablenkungselektroden“ bezeichnet und besteht aus zwei Messingplatten (Kantenlänge: $10 \mathrm{~mm}$ ), die parallel zueinander mit variablem Abstand unterhalb der Aufladungselektrode um die Tropfenkette positioniert sind. Zwischen beiden Platten kann ein zeitlich und näherungsweise räumlich konstantes elektrisches Feld erzeugt werden, womit sich die geladenen Tropfen aus der ungeladenen Tropfenkette herauslenken lassen. Mit der Spannungsquelle (GM24-5KPN-SEC, HiTek Power GmbH) können in Abhängig vom Abstand

${ }^{1}$ Durch die Dissoziation von Wasser ergeben sich $2 \mathrm{H}_{2} \mathrm{O} \rightleftharpoons \mathrm{OH}^{-}+\mathrm{H}_{3}^{+} \mathrm{O}$ als Ladungsträger 33 .

2 Der spezifische, elektrische Widerstand betrug $18 \mathrm{M} \Omega \mathrm{cm}$. Er reduzierte sich durch die Zugabe von $2 \mu \mathrm{l}$ reinen Ammoniaks pro 11 DI Wasser auf einen mittleren Wert von etwa $0,07 \mathrm{M} \Omega \mathrm{cm}$. 


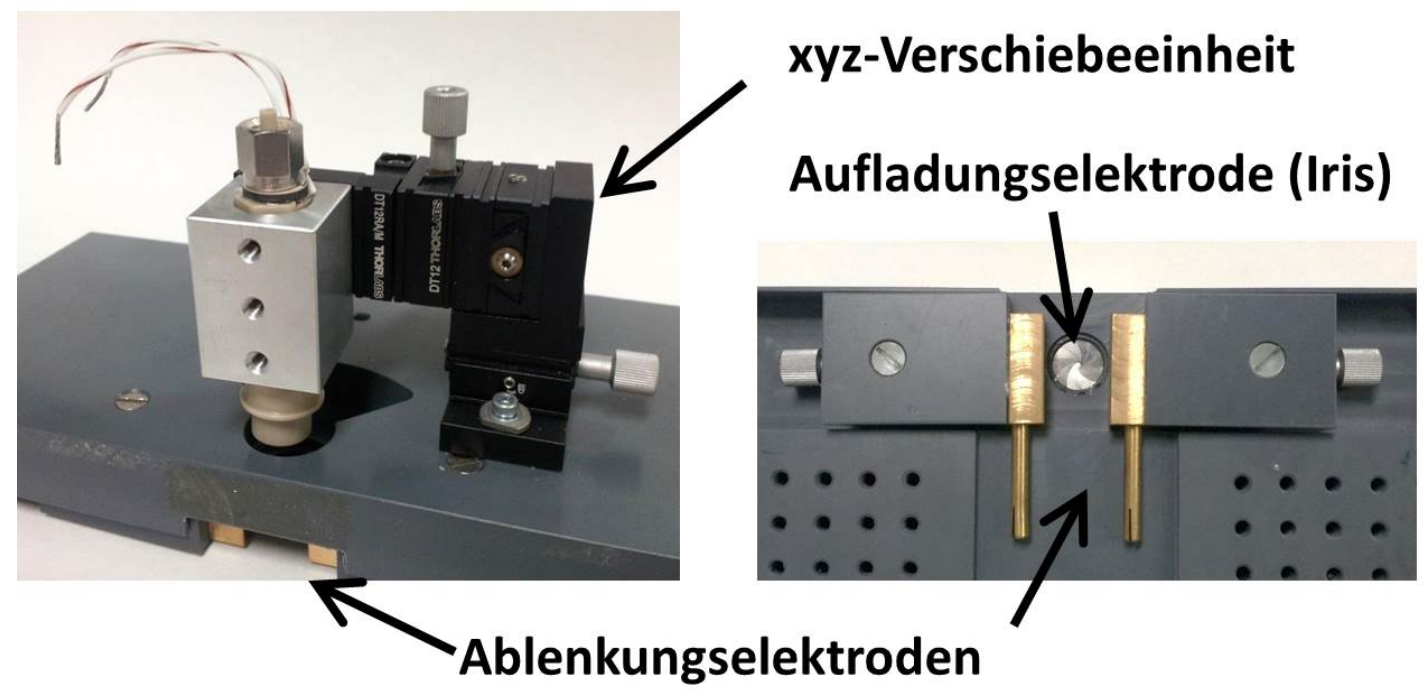

(a)

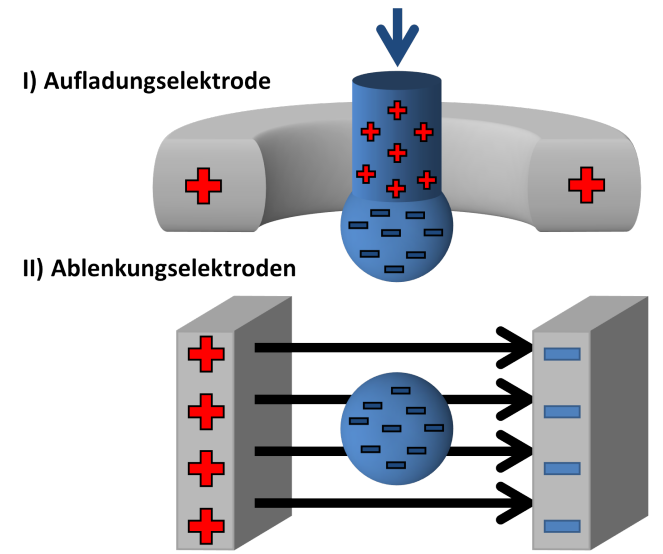

(b)
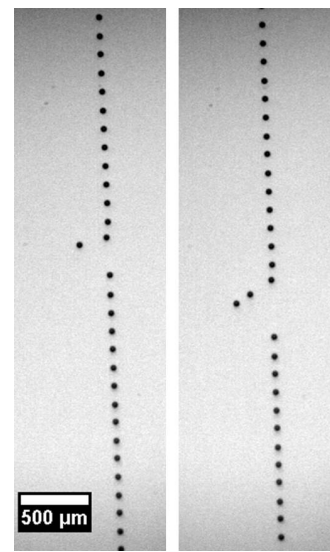

(c)

Abbildung 2.2: (a): Fotos der Halterung der Aufladungs- und Ablenkungselektroden (von der Seite und von unten aufgenommen). Durch eine xyz-Verschiebeeinheit ist die präzise Positionierung der Komponenten gewährleistet. (b): Vergrößerte Darstellung der Aufladung eines einzelnen Tropfens (vgl. Abb. 2.1). Durch einen kurzzeitigen positiven Spannungspuls an der Aufladungselektrode kommt es zu einer Ladungsverschiebung an der Spitze des Flüssigkeitsstrahls, wodurch er polarisiert wird. Der Zeitpunkt und die Dauer werden dabei so gewählt, dass pro Puls jeweils nur ein einziger Tropfen eine negative Nettoladung nach Abtrennung vom Flüssigkeitsstrahl besitzt. Durch ein zeitlich konstantes elektrisches Feld lässt sich der geladene Tropfen seitlich herauslenken. (c): Abhängig vom zeitlichen Verlauf der Aufladung lassen sich z.B. ein, zwei, jeder zehnte oder jeder zweite Tropfen aufladen und anschließend aus der ansonsten neutralen Tropfenkette herauslenken. ( $U \approx 11 \mathrm{~m} \mathrm{~s}^{-1}, D \approx 43 \mu \mathrm{m}$, Bildrate: $40 \mathrm{kfps}$ und Belichtungszeit: $\left.1 \mu \mathrm{s}\right)$ 


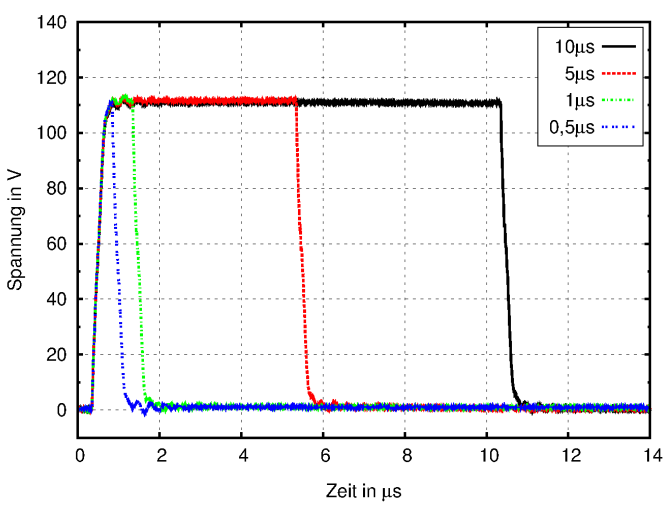

(a)

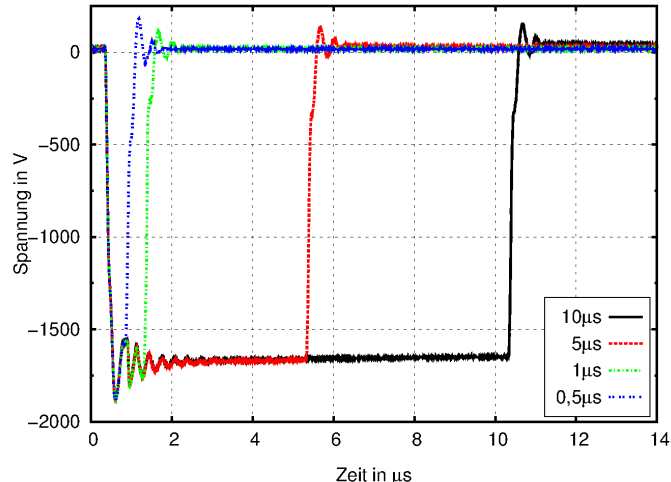

(b)

Abbildung 2.3: Messung der erzeugbaren rechteckförmigen Hochspannungspulse für die Tropfenaufladung. Es sind sowohl (a) positive als auch (b) negative Pulse mit einer Amplitude bis zu ca. $1,75 \mathrm{kV}$ bei variabler Pulslänge möglich. Die Anstiegsund Abfallzeit beträgt jeweils etwa 250 ns. Mit zunehmender Spannungsamplitude zeigen sich vermehrt „Überschwinger“ (siehe auch Anh. E.

der Platten Spannungen von bis zu $10 \mathrm{kV}$ verwendet werden. Die Durchschlagsfestigkeit von Luft beträgt etwa $3 \mathrm{kV} \mathrm{mm}^{-1}$ und beschränkt damit die maximale elektrische Feldstärke.

Anders als Tropfen innerhalb der Tropfenkette erfährt ein einzelner, ausgelenkter Tropfen eine deutlich höhere Luftreibung [91], was schon auf kurzen Strecken $\mathrm{zu}$ einer erheblichen Abbremsung führt (siehe Abb. 2.2c). Entsprechend ist der gesamte Aufbau möglichst kompakt konstruiert. Dabei muss ein ausreichender Abstand zwischen den Komponenten beachtet werden, um Entladungen durch die Verwendung der Hochspannung zu vermeiden. Es ergibt sich eine Gesamtstrecke von $15 \mathrm{~mm}$, bis der Tropfen beide elektrische Komponenten verlässt.

Im Anschluss ist es notwendig, die Tropfenkette in ein Auffangbehältnis umzuleiten, damit nur der Einzeltropfen ungehindert auf dem Substrat auftreffen kann. Durch die räumlich kleinen Beschränkungen erwies sich eine händisch gefaltete Titanfolie von $10 \mu \mathrm{m}$ Dicke als gleichzeitig flexibel aber auch erosionsbeständig genug (gegenüber dem andauernden Tropfeneinschlag), um als Abtrennung zwischen den Einzeltropfen und der Tropfenkette zu fungieren. Typischerweise muss der Tropfen auf einen Abstand $x_{\min }=D+100 \mu \mathrm{m}$ herausgelenkt werden, um ihn zuverlässig von der Tropfenkette separieren zu können. Die aufgesammelte Flüssigkeit wurde anschließend mit einer Spritzenpumpe abgesaugt.

Zur genauen Positionierung aller Komponenten kamen präzise xyz-Verschiebeeinheiten (Thorlabs) zum Einsatz. Der gesamte Aufbau befand sich auf einem 
schwingungsgedämpften optischen Tisch, um mechanische Vibrationen auf ein Minimum zu reduzieren. Zur Aufnahme der Tropfentrajektorie kam dieselbe Technik wie auch schon in Kap. 1.3 beschrieben zum Einsatz. Die sich hiervon unterscheidende Aufnahmetechnik zur Visualisierung des Tropfeneinschlags ist im nachfolgenden Kap. 2.2.2 beschrieben. Die Schaltpläne für die Elektronik, die im Hause von der Elektronischen Werkstatt entworfen und gefertigt wurde, sind in Anh. E zu finden.

\subsubsection{Aufnahmetechnik}

Zur Aufzeichnung des Tropfeneinschlags wurden zwei synchronisierte Aufnahmeeinheiten verwendet, die jeweils aus einer unabhängigen Kamera, Beleuchtungsquelle und Optik bestehen (siehe Abb. 2.1). Der Einschlag erfolgte auf Substrate $e^{3}$ unterschiedlicher Benetzbarkeit: Hydrophil (gereinigter Mikroskopobjektträger aus Glas, Kontaktwinkel $\theta \approx 23^{\circ}$ ), hydrophob (mit Rain- $\mathrm{X}^{\circledR}$ behandelter Mikroskopobjektträger, Kontaktwinkel $\theta \approx 90^{\circ}$ ) und superhydrophob (oberflächenbehandeltes Aluminiumoxid [100], Kontaktwinkel $\theta \approx 135^{\circ}$ ).

Eine Aufnahmeeinheit wurde für die Seitenansicht mittels Hellfeldmikroskopie und einem Mikroskop mit langem Arbeitsabstand (InfiniTube Standard, Infinity) genutzt. Die zweite Aufnahmeeinheit wurde für die Ansicht von unten mittels Dunkelfeldmikroskopie ${ }^{4}$ und einem invertierten Mikroskop (Zeiss, Axiovert CFL40) genutzt. Zur Beleuchtung kamen in beiden Fällen frequenzverdoppelte Doppelpuls Nd:YAG Laser (EverGreen, Quantel Limited) mit einer Wellenlänge $\lambda=532 \mathrm{~nm}$ zum Einsatz. Jeder Laser kann zwei zeitlich unabhängig steuerbare Blitze von etwa 6 ns Dauer erzeugen und damit die Bewegungsunschärfe während der Aufnahme auf ein Minimum reduzieren. Um das Auftreten von Ungleichmäßigkeiten in der Beleuchtung und Interferenzmustern aufgrund der Kohärenz des Laserlichts zu vermindern, wurde das Laserlicht nicht direkt, sondern mittels iLIF und einem zusätzlichen Diffusor für die Seitenansicht verwendet.

Durch eine zeitlich präzise Ansteuerung aller Komponenten mit Funktions- und Verzögerungsgeneratoren (Modell 575, Berkeley Nucleonics Corporation, $t_{\text {Fehler }}<$ $0,8 \mathrm{~ns}$ ) lässt sich der Zeitpunkt der Aufnahme auf die zwei Kameras (Sensicam QE double shutter, PCO AG) synchronisieren, welche in der Lage sind, Doppelbilder kurz hintereinander aufzuzeichnen $(\Delta t \approx 0,5 \mu \mathrm{s})$. Eine ausreichende optische

3 Zwischen den Tropfeneinschlägen wurde das Substrat entweder weiter bewegt oder es wurde gewartet, bis der vorangegangene Tropfen verdunstet war, um reproduzierbar Einschläge auf ein trockenes Substrat zu gewährleisten.

4 Der Bereich oberhalb des Substrats war für eine Beleuchtung aufgrund der Komponenten zur Tropfenerzeugung nicht zugänglich. Außerdem war die Aufnahme von unten nur möglich, wenn das transparente, hydrophile Substrat verwendet wurde. 
Auflösung wurde mittels Mikroskopobjektiven (Olympus LMPLFLN) von 20X für die Seitenansicht und 50X für die Ansicht von unten gewährleistet.

Für die Aufnahme des Tropfeneinschlags mit dieser Methode ist ein hohes Maß an zeitlicher Präzision notwendig. Die zeitliche Ansteuerung der Komponenten ist nachfolgend beschrieben (siehe hierzu auch Abb. 2.4):

1. Ein Funktionsgenerator gibt das „Grundsignal“ als kontinuierliches TTLSignal mit der Frequenz $f$ aus, A).

2. Das Signal wird an einen Verstärker weitergegeben, welcher den piezokeramischen Schallwandler in akustische Schwingungen versetzt, B).

3. Eine neue Aufnahmesequenz, C), wird mit einem Pulsgenerator auf eine steigende Flanke des TTL-Signals gestartet, welcher gleichzeitig eine erneute Auslösung bis zum Ende der Aufnahme verhindert.

4. Nach einer zusätzlichen Verzögerung, D), wird die Ansteuerung der Aufladungselektrode aktiviert, F).

5. Die Pulsbreite und der Zeitpunkt der Tropfenaufladung werden bzgl. A) auf eine maximale Aufladung hin eingestellt, G).

6. Abhängig von der benötigten Zeit, die der Tropfen bis zum Erreichen des Sichtfelds der Kameras benötigt, wird der Beginn der Aufnahme verzögert, E).

7. Die Aufnahmen von der Seite und von unten werden synchron gestartet, H). Jede Aufnahmeeinheit besteht aus einer Kamera und einem Laser (mit „1/2“ gekennzeichnet).

8. Jedes der Doppelbilder wird durch einen Laserblitz beleuchtet. Die Blitzlampen, J) und L), werden zeitlich vor den Q-Switches, I) und K), ausgelöst.

9. Die Zeit zwischen den Doppelbildern, K), lässt sich variabel bis auf etwa $0,5 \mu$ s variieren.

10. Nach Abschluss einer Aufnahmesequenz wird die nächste Sequenz, C), wieder auf das TTL-Signal, A), gestartet. Dabei lassen sich, durch den Laser beschränkt, Repetitionsraten von bis zu $10 \mathrm{~Hz}$ erreichen.

Diese Methode der stroboskopischen Aufnahme ermöglicht es, pro Sequenz zwei Bilder und damit zwei Zeitpunkte des Tropfeneinschlags aufzuzeichnen. Der zeitliche Verlauf während des Aufpralls lässt sich durch Variation des Zeitpunkts von E) untersuchen. Als Resultat können Aufnahmen mit zeitlichen Inkrementen von weniger als 10 ns erzielt werden, was Bildraten von über $100 \mathrm{Mfps}$ entspricht. Die Vorgehensweise zur Bildanalyse ist in Kap. 2.4.1 beschrieben. 


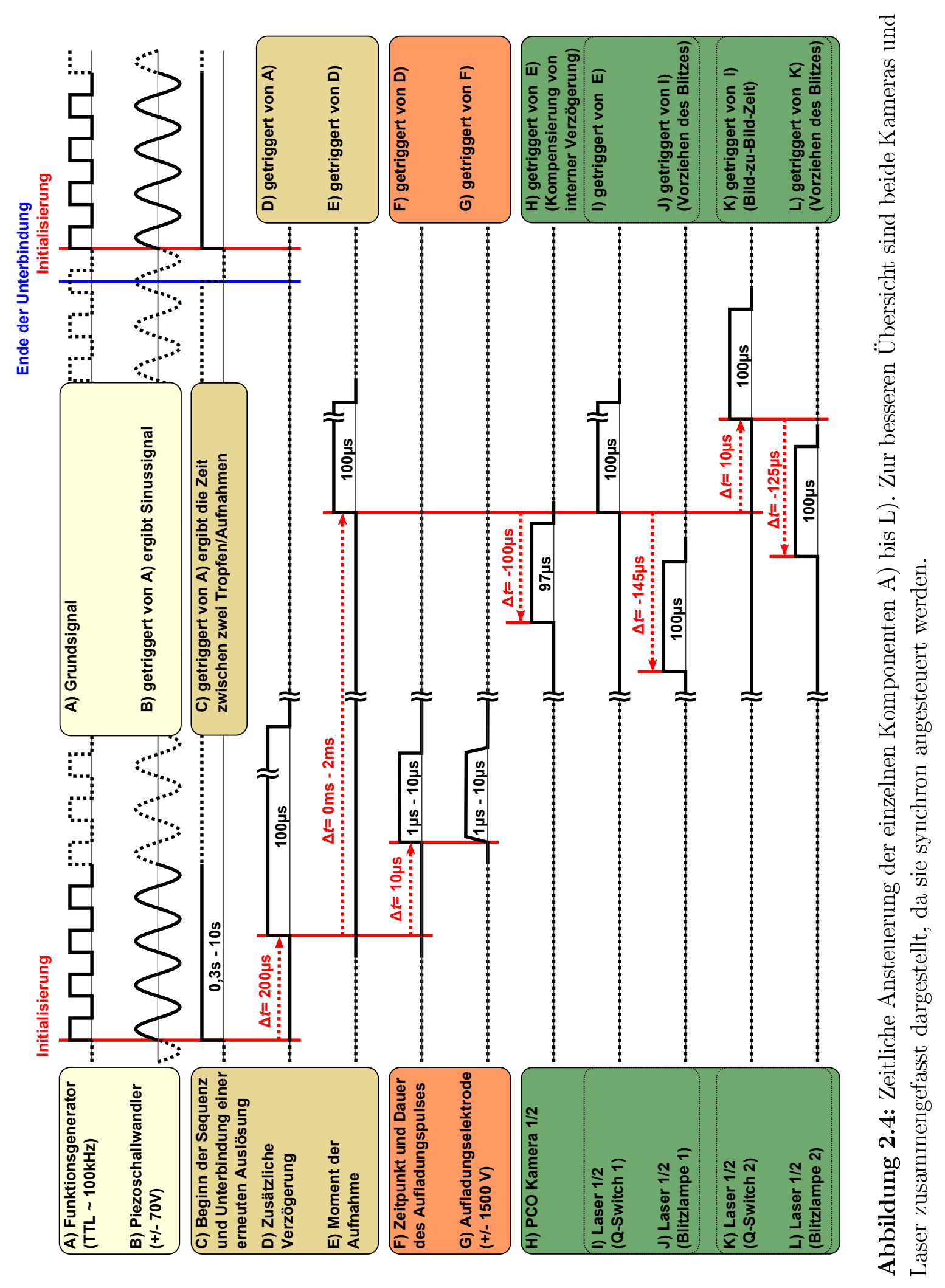





\subsection{Auswertung: Erzeugung einzelner Mikrotropfen}

Ein einzelner Mikrotropfen soll reproduzierbar elektrisch aufgeladen und aus einer Kette monodisperser Tropfen herausgelenkt werden. Anschließend lässt sich ein reproduzierbarer Einzelaufprall auf einem Substrat untersuchen. Die Ergebnisse und Schlussfolgerungen in diesem Kapitel beziehen sich auf Experimente, die mit der $17 \mu \mathrm{m}$ Siliziumdüse entsprechend Kap.1 erzielt wurden. Die Funktionsweise der Erzeugung einer Tropfenkette definierter Tropfengröße und -geschwindigkeit wurden dort bereits behandelt.

\subsubsection{Prozess der Tropfenaufladung}

Wird ein Tropfen elektrisch aufgeladen, so wird in ihm die Ladungsträgerdichte (positiv oder negativ) erhöht. Durch die Coulomb-Abstoßung zwischen zwei gleichen Ladungen $Q$ entsteht eine nach außen wirkende Kraft, die der nach innen gerichteten Oberflächenspannung $\sigma$ entgegenwirkt. Überwiegt die elektrostatische Abstoßung, so wird der Tropfen instabil und zerfällt solange in kleinere Teiltropfen, bis die Oberflächenspannung die Abstoßung wieder kompensiert. Durch die Abschätzung beider Effekte zeigte Rayleigh [101], wie die maximale Ladungsträgeranzahl in einem Tropfen bestimmt werden kann — das sogenannte „Rayleigh-Limit":

$$
Q_{\text {Ray }}=8 \pi \sqrt{\varepsilon_{0} \sigma R^{3}},
$$

wobei $\varepsilon_{0}$ die Permittivität des Vakuums und $R$ der Tropfenradius ist. Während der kontinuierlichen Erzeugung einer monodispersen Tropfenkette (entsprechend Kap. 1), lässt sich ein einzelner Tropfen aufladen, indem für den Zeitraum seiner Ablösung vom Flüssigkeitsstrahl ein elektrisches Feld eine Verschiebung von Ladungsträgern im Flüssigkeitsstrahl hervorruft 33, 102 104. Dazu befindet sich um die Position der Tropfenablösung eine ringförmige Aufladungselektrode. Da der Strahl über die Düse leitend mit dem Schutzleiter verbunden ist, verursacht ein Spannungspuls an der Aufladungselektrode kurzzeitig eine entgegengesetzte Polarisierung der Spitze des Strahls. Endet der Spannungspuls nach der Tropfenablösung, so ist nur dieser Tropfen geladen und der nachfolgende Tropfen wieder neutral. Ähnlich einem Kugelkondensator lässt sich dem Tropfen eine Kapazität $C=4 \pi \varepsilon_{0} R$ zuordnen. Mit der Aufladungsspannung $U_{\text {aufl }}$ und der relativen Permittivität der umgebenden Luft $\varepsilon_{\mathrm{r}} \approx 1$ kann so die Ladungsmenge bestimmt werden

$$
Q=4 \pi \varepsilon_{0} \varepsilon_{\mathrm{r}} R U_{\text {aufl }}
$$


Dieses Modell lässt sich erweitern [82, 105, 106], indem die Aufladungselektrode und der Flüssigkeitsstrahl als Zylinderkondensator modelliert werden. Dieser Kondensator wird über einen Widerstand aufgeladen, der dem Teil des Flüssigkeitsstrahls entspricht, welcher sich zwischen der Aufladungselektrode und der Düse befindet. Die Ladungsmenge pro Einheitsmasse $m$ beträgt in diesem Fall

$$
Q / m=\frac{2 \varepsilon_{0} \varepsilon_{\mathrm{r}} U_{\text {aufl }}}{\rho_{\mathrm{l}} a^{2} \ln \left(R_{\mathrm{aufl}} / a\right)}
$$

mit der Dichte der Flüssigkeit $\rho_{\mathrm{l}}$, dem Radius des Flüssigkeitsstrahls $a$ und dem Radius des Aufladungselektrode $R_{\text {aufl }}$. Diese Ladungsmenge wird im Fall einer unendlich lange Aufladungsdauer erreicht. Abhängig von der tatsächlichen Dauer der Aufladung $t$ beträgt die Ladungsmenge pro Tropfen

$$
Q(t)=\frac{8}{3} \pi \cdot \frac{R^{3} \varepsilon_{0} \varepsilon_{\mathrm{r}} U_{\mathrm{aufl}}}{a^{2} \ln \left(R_{\mathrm{aufl}} / a\right)} \cdot\left(1-e^{-t / \tau}\right)
$$

wobei die Zeitkonstante der Aufladung $\tau$ durch die Leitfähigkeit der Flüssigkeit gegeben ist. Da sich pro Periode der akustischen Anregung $T=1 / f$ je ein Tropfen (mit einer Größe entsprechend einer Wellenlänge $\lambda$ der akustischen Anregung) vom Flüssigkeitsstrahl ablöst, muss die Aufladungsdauer stets $t \leq T$ sein, damit keine weiteren Tropfen aufgeladen werden. Außerdem muss der Aufladungspuls für eine Dauer von $t<T$ zum Ende der Tropfenablösung geschehen, da ansonsten ein Ladungsaustausch mit dem Flüssigkeitsstrahl erfolgt und eine geringere Ladungsmenge auf dem sich ablösenden Tropfen verbleibt. Hierbei gilt die Annahme, dass die Zeit für den Ladungstransport/-ausgleich im Vergleich zur Zeit, die sich das zu ladende Flüssigkeitssegment durch die Aufladungselektrode bewegt, klein ist 106.

Zur Bestimmung der Zeitkonstante lässt sich der Zusammenhang $\tau=R_{\mathrm{R}} C$ mit dem Widerstand $R_{\mathrm{R}}$ benutzen [105, über den die Kapazität $C$ aufgeladen wird. Der Widerstand ergibt sich aus dem spezifischen elektrischen Leitwert $\sigma_{\mathrm{R}}$ der Flüssigkeit und dem Radius $a$ und der Länge $L$ des Flüssigkeitsstrahls, der außerhalb der Aufladungselektrode ist. Die Zeitkonstante ergibt sich dann zu

$$
\tau=8 / \pi^{2} \cdot \varepsilon_{0} \varepsilon_{\mathrm{r}} / \sigma_{\mathrm{R}} \cdot(L / a)^{2} / \ln \left(R_{\mathrm{aufl}} / a\right) \quad .
$$

Die bisher gemachten Annahmen über den Prozess der Aufladung sollen im Folgenden experimentell untersucht werden. Aus der Distanz der Auslenkung des geladenen Tropfens nach einer Zeit $t$ aufgrund des elektrischen Ablenkfelds lässt sich die Menge der auf den Tropfen übertragenen Ladungen durch eine Vereinfachung 
der Bewegungsgleichung in $\mathrm{x}$-Richtung abschätzen als

$$
\begin{aligned}
\ddot{x} & =Q E_{x, \mathrm{abl}} / m \\
\dot{x} & =Q E_{x, \mathrm{abl}} t / m \\
x & =Q E_{x, \mathrm{abl}} t^{2} / 2 m \\
\Rightarrow Q & =\frac{2 m \Delta x_{\mathrm{abl}}}{E_{x, \mathrm{abl}} t^{2}},
\end{aligned}
$$

mit der Tropfenmasse $m$ und der elektrischen Feldstärke $E_{x \text {, abl }}$ zwischen den Ablenkungselektroden.

In Abb. 2.5 sind die experimentellen Messergebnisse für einen Tropfen mit $37,5 \mu \mathrm{m}$ Durchmesser und einer Geschwindigkeit von $26 \mathrm{~m} \mathrm{~s}^{-1}$ aufgezeigt. Als Flüssigkeit wurde DI Wasser mit einer Dichte von $\rho_{1}=1000 \mathrm{~kg} \mathrm{~m}^{-3}$ verwendet. Der Radius der Aufladungselektrode betrug $R_{\text {auf }}=0,5 \mathrm{~mm}$, der Radius des Flüssigkeitsstrahl ${ }^{5} a=8,4 \mu \mathrm{m}$, die Länge des Flüssigkeitsstrahls ${ }^{6} 0,5 \mathrm{~mm}$ und der spezifische Leitwert ${ }^{7}$ der Flüssigkeit $\sigma_{\mathrm{R}}=1,48 \mathrm{mS} \mathrm{m}^{-1}$. Da die Frequenz der akustischen Anregung $f=210 \mathrm{kHz}$ (entspricht einer Periode von etwa 4,76 us) betrug, wurde die Dauer der Aufladung $t$ bei einer Spannung von $U_{\text {aufl }}=300 \mathrm{~V}$ zwischen $0 \mu \mathrm{s}$ und $4,7 \mu \mathrm{s}$ variiert. Für ein konstantes elektrisches Ablenkungsfeld von $E_{x \text {, abl }}=0,4 \mathrm{kV} \mathrm{mm}^{-1}$ werden die jeweiligen Ablenkungen $\Delta x_{\mathrm{abl}}$ in den Hochgeschwindigkeitsaufnahmen bestimmt und mittels Gl.(2.4) in die übertragene Ladungsmenge umgerechnet. Ein Fit der Messdaten mit $Q(t)=Q_{\max } \cdot\left(1-e^{-t / \tau}\right)$ ermöglicht die Bestimmung der maximal möglichen Ladungsmenge zu $Q_{\max }=1,1 \times 10^{-13} \mathrm{C}$ und der Zeitkonstante zu $\tau=2,8 \mu \mathrm{s}$. Die maximal mögliche Ladungsmenge $Q_{\text {Ray }}=1,63 \times 10^{-12} \mathrm{C}$ (bzw. in Elementarladungen $n=Q_{\text {Ray }} / e=1,0 \times 10^{7}$ mit $e=1,602 \times 10^{-19} \mathrm{C}$ ) wurde nicht erreicht. In der Abbildung ist ebenfalls die erwartete Kurve mit den theoretisch nach Gl. 2.1 und (2.3) bestimmten Größen $Q_{\max }=5,1 \times 10^{-13} \mathrm{C}$ und der Zeitkonstante $\tau=4,3 \mu$ s gegeben.

${ }^{5}$ Es wird angenommen, dass der Flüssigkeitsstrahl nach Verlassen der Düse den gleichen Radius wie die Düse hat.

${ }^{6}$ Wie in Kap. 1.4 .4 beschrieben, konnten kleinere Zerfallslängen messtechnisch nicht erfasst werden. Die tatsächliche Zerfallslänge kann entsprechend kürzer sein.

7 Durch die Zugabe einer geringen Menge Ammoniak wurde der Leitwert des Wassers erhöht, um eine bessere Kontrollierbarkeit der Tropfenaufladung zu gewährleisten. Der gemittelte Leitwert wurde durch Probenentnahmen über einen Zeitraum von zwei Wochen bestimmt, um Schwankungen bei der Messung der Ammoniakkonzentration zu kompensieren. Während der Tropfenerzeugung im Experiment sind jedoch noch weitere Schwankungen zu erwarten, die zum einen durch mögliche Konzentrationsunterschiede aufgrund der Länge des verwendeten Schlauch- und Anschlusssystems, als auch durch eine hohe Flüchtigkeit des gelösten Ammoniaks verursacht sind. 


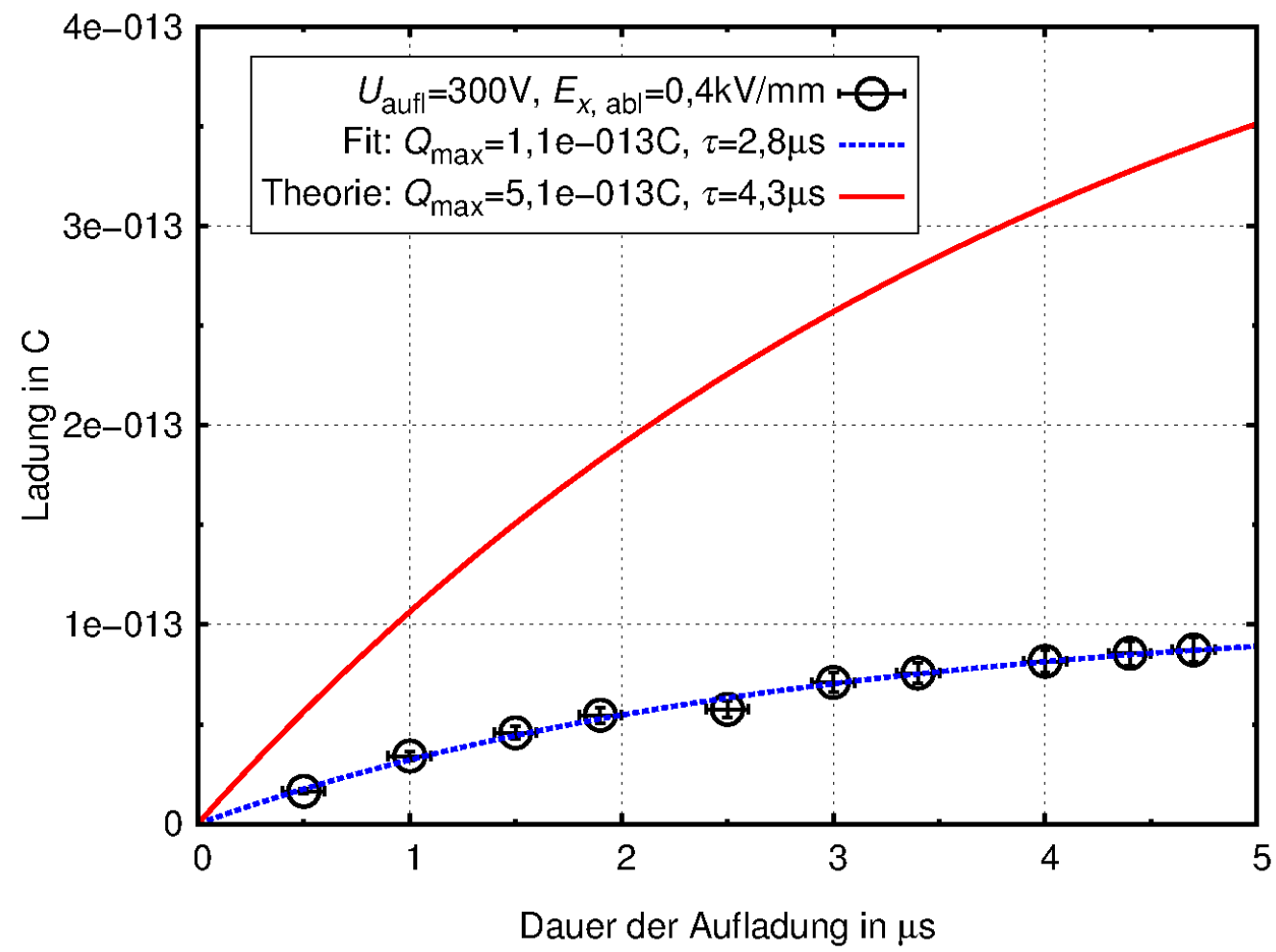

Abbildung 2.5: Vergleich der auf den Tropfen übertragenen Ladungsmenge $Q$ als Funktion der Aufladungsdauer $t$. Die experimentellen Messdaten (Kreissymbole) lassen sich mit dem Modell (gestrichelte Linie) nach Gl. (2.4) beschreiben. Die nach Gl. 2.1 und 2.3 theoretisch erwartete Ladungsmenge pro Tropfen (durchgezogene Linie) weicht deutlich vom Experiment ab.

Unter Anbetracht der Reduzierung des Aufladungsprozesses auf das Modell eines Zylinderkondensators und die Vereinfachung der Bewegungsgleichung war diese Abweichung zu erwarten. Die Hauptgründe der Abweichung sind die folgenden: Die Aufladungselektrode ist kein ausgedehnter Zylinder, sondern eine dünne Iris, was ein verändertes elektrisches Feld zur Folge hat. Durch die Verdeckung des Flüssigkeitsstrahls durch die Iris konnte sie nicht exakt an der Position des Strahlzerfalls angebracht werden. Sie befand sich, begründet durch die Ergebnisse in Abb. 1.14, in einem konstanten Abstand von $1 \mathrm{~mm}$ unterhalb der Düse. Damit kann die tatsächliche elektrische Feldstärke der Aufladung kleiner als die gemessene sein. Die elektrische Feldstärke des zeitlich konstanten Ablenkungsfeldes ließ sich nicht während des Experiments bestimmen. Entsprechend zuvor bestimmter Reglerstellungen an der Spannungsquelle konnte die Spannung auf etwa $5 \%$ genau eingestellt werden. In der Bewegungsgleichung werden der Einfluss der Luftreibung, eine initiale Geschwindigkeitskomponente in x-Richtung 
(durch eine vorangegangene Beschleunigung außerhalb des Sichtbereiches der Aufnahme) und die sich räumlich ändernde elektrische Feldstärke der Ablenkung vernachlässigt. Die Bestimmung der Zerfallslänge war messtechnisch limitiert. Die tatsächliche Länge kann kürzer sein und hätte eine kleinere Zeitkonstante der Aufladung $\tau$ zur Folge. Durch die Flüchtigkeit des zugegebenen Ammoniaks kann sich die tatsächliche Leitfähigkeit der Flüssigkeit sowohl zeitlich als auch räumlich unterscheiden.

Versuche mit drei weiteren Kombinationen aus Tropfengrößen und -geschwindigkeiten und Aufladungsspannungen weisen vergleichbare Abweichungen auf, siehe Abb. 2.6. In allen Fällen ist jedoch eine gute Beschreibung der mit der Aufladungsdauer exponentiell steigenden Ladungsmenge zu sehen.

Der in Abb. 2.7 dargestellte Überblick zeigt die Abweichungen quantitativ. Für drei Kombinationen aus Tropfengröße und -geschwindigkeit ist ein nahezu linearer Anstieg der maximal übertragenen Ladungsmenge mit der Aufladungsspannung zu sehen. Jedoch liegen die experimentell bestimmten Werte stets unterhalb der theoretisch erwarteten. Insbesondere ist die maximal übertragbare Ladungsmenge für den $43 \mu \mathrm{m}$ Tropfen kleiner als für den $40 \mu \mathrm{m}$ Tropfen. Ausgehend von Gl. 2.1) wäre das Gegenteil zu erwarten. Von den zuvor genannten Gründen für die Abweichung ist in diesem Fall die unterschiedliche Tropfengeschwindigkeit und damit die Position der Tropfenablösung hervorzuheben. Wenn sich die Aufladungselektrode nicht genau an der Ablösungsposition befindet, ist das elektrische Feld und damit die übertragene Ladungsmenge geringer. Die theoretisch erwartete maximale Ladung liegt in allen Fällen um einen Faktor zwei bis fünf über der im Experiment bestimmten. Hierbei ist eine Tendenz $\mathrm{zu}$ geringer werdenden maximalen Werten mit steigender Aufladungsspannung zu beobachten. Möglicherweise kommt es durch die begrenzte Leitfähigkeit der Flüssigkeit zu einer Limitierung der verfügbaren Ladungsmenge in der Spitze des Flüssigkeitsstrahls.

Es bleibt jedoch festzuhalten, dass Gl. (2.1) und (2.3) den Prozess der Aufladung im Allgemeinen qualitativ gut beschreiben, jedoch die in der Modellannahme nicht berücksichtigten Faktoren zu einer quantitativen Unterschätzung beider Größen führen. 

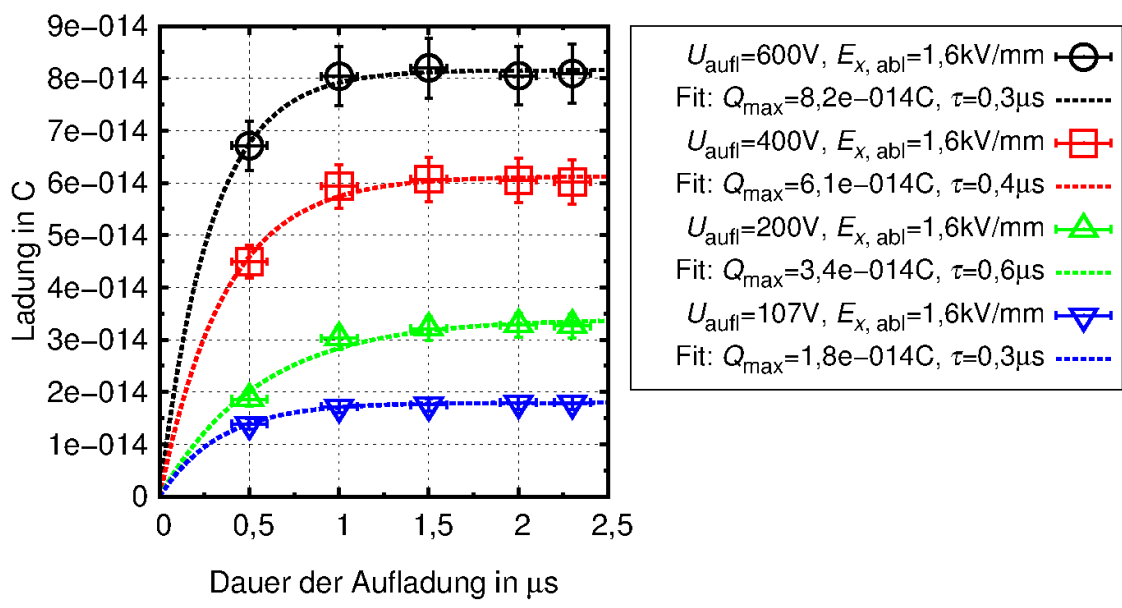

(a) $D=31 \mu \mathrm{m}, U=30 \mathrm{~m} \mathrm{~s}^{-1}$

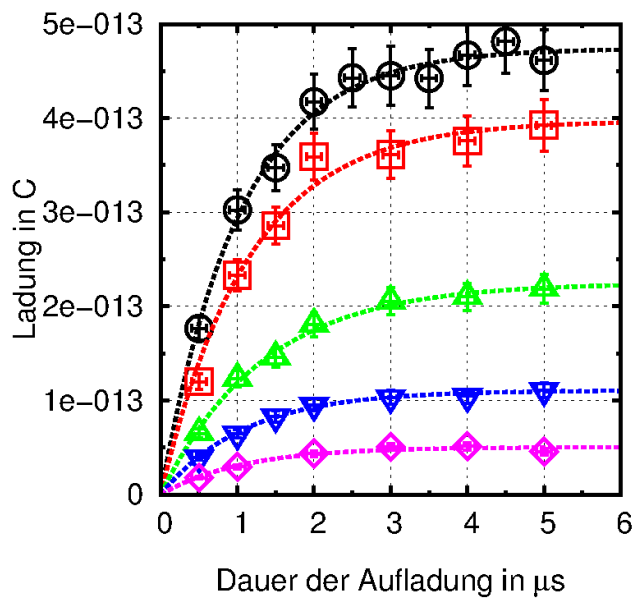
$U_{\text {auf }}=600 \mathrm{~V}, E_{x, \mathrm{abl}}=1,0 \mathrm{kV} / \mathrm{mm}$ Fit: $Q_{\max }=4,7 \mathrm{e}-013 \mathrm{C}, \tau=1,0 \mu \mathrm{s}$........ $U_{\text {aufl }}=410 \mathrm{~V}, E_{X, a b l}=1,0 \mathrm{kV} / \mathrm{mm}$ ' Fit: $Q_{\max }=4,0 \mathrm{e}-013 \mathrm{C}, \tau=1,1 \mu \mathrm{s}$ $U_{\text {aufl }}=200 \mathrm{~V}, E_{x, \text { abl }}=1,0 \mathrm{kV} / \mathrm{mm} \wedge$ Fit: $Q_{\max }=2,2 \mathrm{e}-013 \mathrm{C}, \tau=1,3 \mu \mathrm{s}$ $U_{\text {aufl }}=100 \mathrm{~V}, E_{x, a b l}=1,6 \mathrm{kV} / \mathrm{mm} \mapsto$ Fit: $Q_{\max }=1,1 \mathrm{e}-013 \mathrm{C}, \tau=1,1 \mu \mathrm{s}$ $U_{\text {auf }}=50 \mathrm{~V}, E_{x, \text { abl }}=1,6 \mathrm{kV} / \mathrm{mm} \mapsto$ Fit: $Q_{\max }=5,0 \mathrm{e}-014 \mathrm{C}, \tau=1,1 \mu \mathrm{s}$

(b) $D=40 \mu \mathrm{m}, U=30 \mathrm{~m} \mathrm{~s}^{-1}$

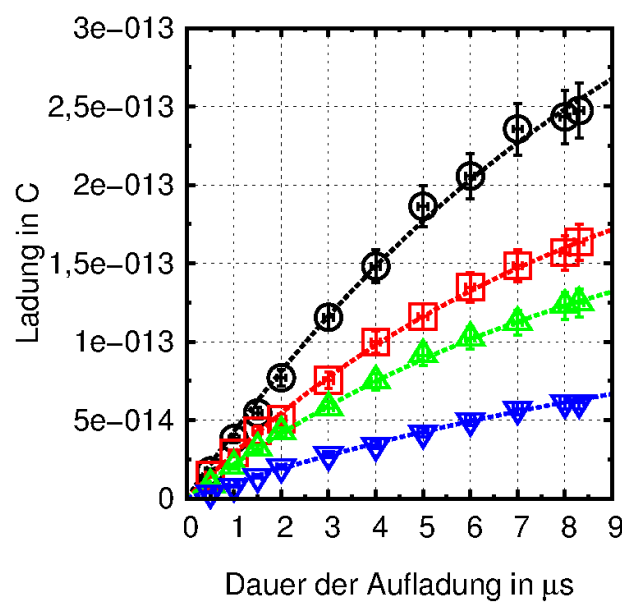

$U_{\text {aufl }}=1080 \mathrm{~V}, E_{x, \text { abl }}=0,7 \mathrm{kV} / \mathrm{mm}$ Fit: $Q_{\max }=4,6 \mathrm{e}-013 \mathrm{C}, \tau=10,2 \mu \mathrm{s}$....... $U_{\text {aufl }}=570 \mathrm{~V}, E_{x, \text { abl }}=1,1 \mathrm{kV} / \mathrm{mm}$ 口 Fit: $Q_{\max }=2,7 \mathrm{e}-013 \mathrm{C}, \tau=8,8 \mu \mathrm{s}$ $U_{\text {aufl }}=380 \mathrm{~V}, E_{x, \text { abl }}=1,3 \mathrm{kV} / \mathrm{mm} \mapsto \triangle$ Fit: $Q_{\max }=2,1 \mathrm{e}-013 \mathrm{C}, \tau=9,1 \mu \mathrm{s}$ $U_{\text {aufl }}=190 \mathrm{~V}, E_{x, \text { abl }}=1,8 \mathrm{kV} / \mathrm{mm} \mapsto \nabla$ Fit: $Q_{\max }=1,3 e-013 \mathrm{C}, \tau=13,1 \mu \mathrm{s}$.......

(c) $D=43 \mu \mathrm{m}, U=23 \mathrm{~m} \mathrm{~s}^{-1}$

Abbildung 2.6: Die Ladungsmenge $Q$ pro Tropfen in Abhängigkeit von der Dauer $t$ und von der Amplitude der Aufladungsspannung $U_{\text {aufl }}$. Für verschiedene Tropfengrößen und -geschwindigkeiten (siehe Bildunterschrift) lässt sich der Aufladungsprozess mit Gl. 2.4 beschreiben (gestrichelte Linien). 


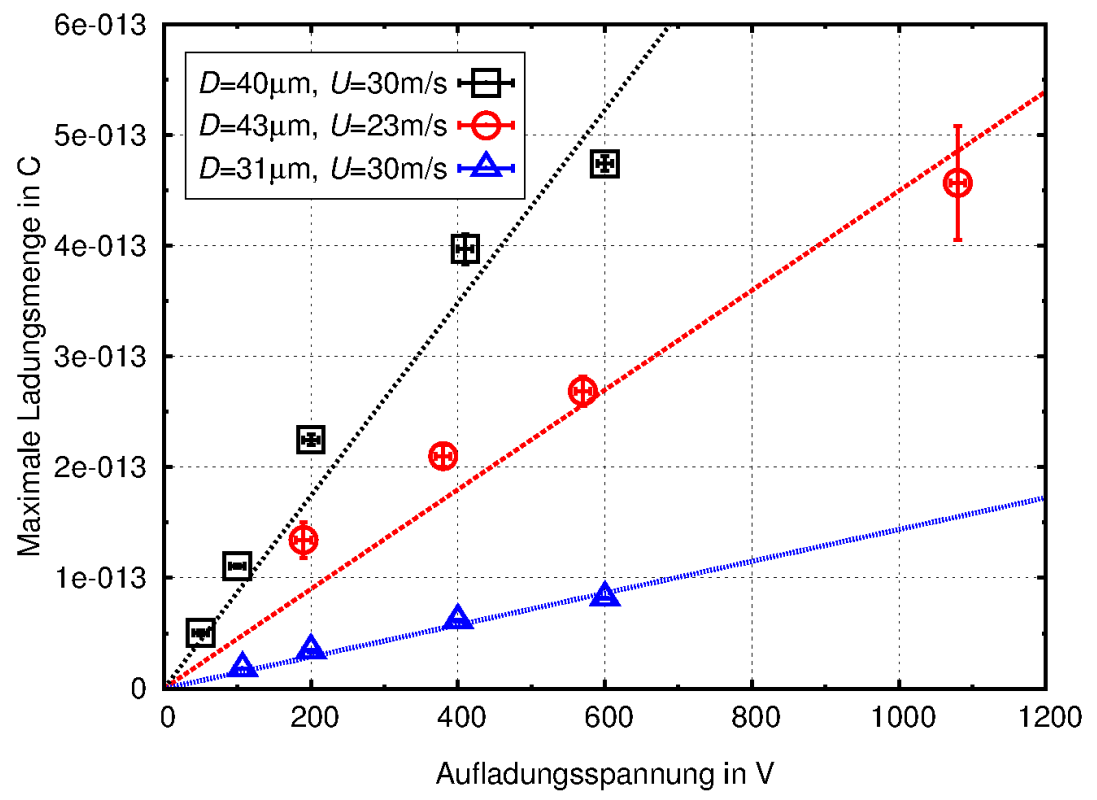

(a)

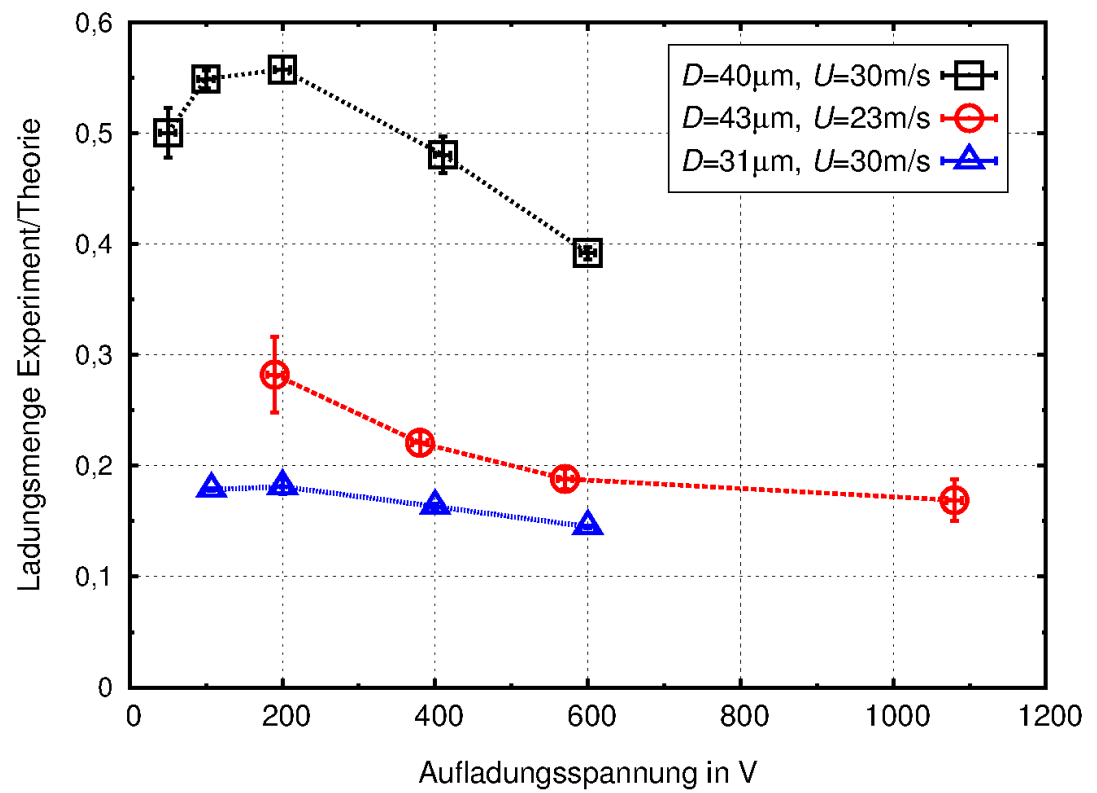

(b)

Abbildung 2.7: (a): Bestimmung der maximalen Ladungsmenge $Q_{\max }$ pro Tropfen (aus Abb. 2.6) als Funktion der Aufladungsspannung $U_{\text {aufl }}$ für drei verschiedene Tropfengrößen und -geschwindigkeiten. Es zeigt sich ein nahezu linearer Anstieg (gestrichelte Linien). (b): Verhältnis der nach Gl. (2.1) berechneten und der experimentell bestimmten maximalen Ladungsmenge $Q_{\max }$. Die experimentellen Werte liegen unterhalb der theoretischen Erwartung mit einer Tendenz zu abnehmenden maximalen Ladungsmengen mit steigender Aufladungsspannung. 


\subsubsection{Bewegungsgleichungen im elektrischen Feld}

Zur Beschreibung der Tropfentrajektorie werden im Folgenden die auf einen elektrisch geladenen Tropfen wirkenden Kräfte in einem Modell betrachtet. Das im Weiteren verwendeten Koordinatensystem ist in Abb. 2.8 dargestellt. Auf elektrisch geladene Tropfen wirken in verwendeten Aufbau drei relevante Kräfte:

1. Gravitation:

$$
\vec{F}_{\mathrm{G}}=-m g \vec{e}_{y}
$$

mit der Tropfenmasse $m$ und der Erdbeschleunigung $g=9,81 \mathrm{~m} \mathrm{~s}^{-2}$, die entlang des Einheitsvektors in negativer y-Richtung auf den Schwerpunkt wirkt.

2. Elektrisches Ablenkungsfeld:

$$
\vec{F}_{\mathrm{E}}=Q E(y) \vec{e}_{x}
$$

mit dem in $\mathrm{x}$-Richtung räumlich konstanten elektrischen Feld $E(y)$ zwischen den Ablenkungselektroden. Die Änderungen in $\mathrm{x}$-Richtung werden als vernachlässigbar klein angenommen.

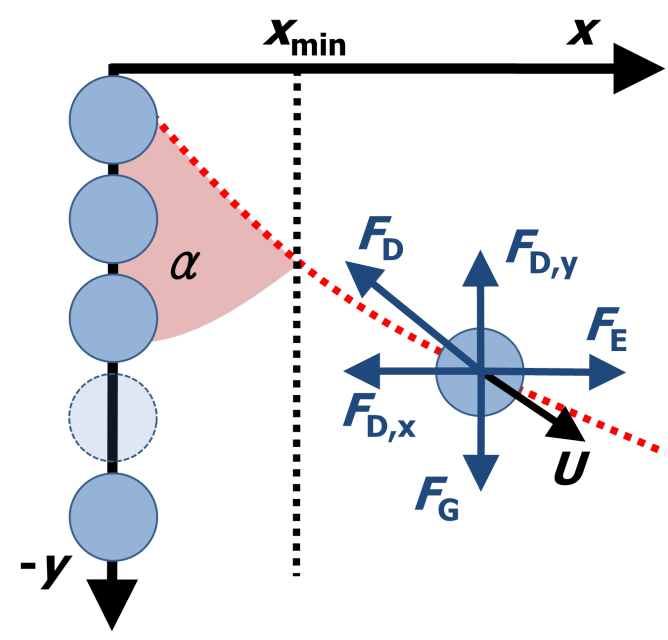

Abbildung 2.8: Koordinatensystem der ausgelenkten Tropfentrajektorie (rote gestrichelte Linie). Die Tropfenkette bewegt sich entlang der negativen y-Achse. Ein einzelner elektrisch geladener Tropfen wird durch das elektrische Ablenkungsfeld unter dem Winkel $\alpha$ abgelenkt und es verbleibt eine Leerstelle in der Tropfenkette (gestrichelter Kreis). Es wirken drei Kräfte: Gravitation $F_{\mathrm{G}}$, Elektrisches Ablenkungsfeld $F_{\mathrm{E}}$ und die Luftreibung $F_{\mathrm{D}}$. Die schwarze gestrichelte Linie charakterisiert einen Mindestabstand $x_{\min }$, der erreicht werden soll. 
3. Reibung mit der umgebenden Luft8

$$
\vec{F}_{\mathrm{D}}=-\frac{A}{2} \rho_{\mathrm{g}} \cdot C_{\mathrm{D}}(U) \cdot U^{2} \cdot \frac{\vec{U}}{U}
$$

mit der projizierten Fläche des Tropfens $A=\pi R^{2}$ und der Dichte des Gases (hier Luft) $\rho_{\mathrm{g}}=1,3 \mathrm{~kg} \mathrm{~m}^{-3}$. Der Faktor $C_{\mathrm{D}}(U)$ ist der Strömungswiderstandskoeffizient und berechnet sich für einen einzelnen kugelförmigen Tropfen 106 aus

$$
C_{\mathrm{D}, \text { einzel }}(U) \approx 24 / R e+1 /(1+\sqrt{R e})+0.4
$$

mit der Reynolds-Zahl Re $=\rho_{\mathrm{g}} D U / \mu_{\mathrm{g}}$ bezogen auf die Viskosität des umgebenden Gases (hier Luft) $\mu_{\mathrm{g}} \approx 18 \times 10^{-6}$ Pas mit dem Tropfendurchmesser $D$ und der Tropfenrelativgeschwindigkeit $U=|\vec{U}|$. Die Reibungskraft ist abhängig von der auf das Gas bezogenen Relativgeschwindigkeit. Entsprechend verschwindet die Reibungskraft, falls das Gas eine von Betrag und Richtung identische Geschwindigkeit wie der Tropfen hat. Außerdem können ihre $\mathrm{x}$ - und $\mathrm{y}$-Komponenten nicht einfach aus den $\mathrm{x}$ - und y-Komponenten von $\vec{U}$ berechnet werden, sondern folgen mit dem Winkel $\alpha$ (gemessen im Uhrzeigersinn ausgehend von der negativen $\mathrm{y}^{-}$ Achse, vgl. Abb. 2.8 entsprechend

$$
\begin{aligned}
& F_{\mathrm{D}, x}=-\sin \alpha \cdot\left|\vec{F}_{\mathrm{D}}\right|=-\frac{U_{x}}{U} \cdot\left|\vec{F}_{\mathrm{D}}\right| \\
& F_{\mathrm{D}, y}=-\cos \alpha \cdot\left|\vec{F}_{\mathrm{D}}\right|=-\frac{U_{y}}{U} \cdot\left|\vec{F}_{\mathrm{D}}\right| .
\end{aligned}
$$

Das Gleichungssystem der Tropfentrajektorie ergibt sich zu

$$
m \ddot{\vec{x}}=\vec{F}_{\mathrm{G}}+\vec{F}_{\mathrm{E}}+\vec{F}_{\mathrm{D}}
$$

und mit Gl.2.5-2.10 $\mathrm{zu}$

$$
\begin{aligned}
& \ddot{x}=\frac{Q E(y)}{m}-\frac{A}{2} \cdot \frac{\rho_{\mathrm{g}}}{m} \cdot C_{\mathrm{D}}(U) \cdot U \cdot U_{x} \\
& \ddot{y}=-g \quad-\frac{A}{2} \cdot \frac{\rho_{\mathrm{g}}}{m} \cdot C_{\mathrm{D}}(U) \cdot U \cdot U_{y}
\end{aligned}
$$

Bevor das gesamte Gleichungssystem gelöst wird, werden die zwei Fälle eines Tropfens innerhalb der Tropfenkette und eines herausgelenkten Tropfens nahe der Tropfenkette getrennt betrachtet.

8 Die Reibungskraft wirkt stets entgegengesetzt zum Geschwindigkeitsvektor der Bewegung. 


\section{Einzeltropfen innerhalb der Tropfenkette}

Die Reibungskraft, die auf einen Tropfen innerhalb der Tropfenkette wirkt, unterscheidet sich erheblich von der eines isolierten Tropfens entsprechend Gl. (2.8). Grund dafür ist die durch die Viskosität der umgebenden Luft angetriebene Strömung entlang des Flüssigkeitsstrahls bzw. Tropfenkette [107]. Des Weiteren bewegt sich jeder Tropfen im „Windschatten“ seiner Vorgänger, d.h. die projizierte Fläche der Anströmung bzw. die Anströmungsgeschwindigkeit weichen erheblich ab. In der Literatur werden für diesen Fall häufig empirische Annäherungen für den Strömungswiderstandskoeffizient $C_{\mathrm{D}}$, Kette verwendet. Kim et al. 91 verifizierten die ursprünglichen Ergebnisse von Mulholland et al. 108 unter Verwendung von

$$
C_{\mathrm{D}, \text { Kette }}{ }^{-p}=C_{\mathrm{D}, 0}^{-p}+C_{\mathrm{D}, \infty}^{-p}
$$

mit der empirisch bestimmten Konstante $p=0,678$. Es wird eine unendlich ausgedehnte Kette von Tropfen mit Durchmesser $D$ und mit einem Abstand ${ }^{9} l_{\mathrm{s}}$ untereinander angenommen. $C_{\mathrm{D}, 0}$ beschreibt den asymptotischen Grenzfall für sich berührende Tropfen (wenn $l_{\mathrm{s}} / D \rightarrow 1$ ) und $C_{\mathrm{D}, \infty}$ für unendlich von einander entfernte Tropfen (wenn $l_{\mathrm{s}} / D \rightarrow \infty$ ). Letzterer Term ist identisch zu $C_{\mathrm{D}}$, einzel aus Gl. (2.8). Der erste Term lässt sich schreiben als

$$
C_{\mathrm{D}, 0}=C_{\mathrm{D}, 0}^{\prime}+\frac{e}{R e} \cdot\left(\frac{l_{\mathrm{s}}}{D}-1\right)
$$

mit der empirischen Konstante $e=43$ und dem Strömungswiderstandskoeffizient $C_{\mathrm{D}, 0}^{\prime}\left(\right.$ wenn $\left.l_{\mathrm{s}} / D=1\right)$, was wiederum durch

$$
C_{\mathrm{D}, 0}^{\prime}{ }^{-p}=C_{\mathrm{D}, \text { Strahl }^{-p}}-C_{\mathrm{D}, \infty}{ }^{-p}
$$

ausgedrückt werden kann. $C_{\mathrm{D}}$, Strahl $=0,755 /$ Re ist der Strömungswiderstandskoeffizient eines unendlich ausgedehnten Zylinders bzw. des Flüssigkeitsstrahls [108]. In Abb. 2.9 ist der Verlauf des Strömungswiderstandskoeffizienten als Funktion der Reynolds-Zahl $R e=\rho_{\mathrm{g}} D U / \mu_{\mathrm{g}}$ dargestellt. Der Vergleich zeigt deutlich den verringerten Luftwiderstand von Tropfen innerhalb der Tropfenkette gegenüber dem eines isolierten Einzeltropfens. Für kleine Tropfen ( $k a_{\max }$, siehe Kap. 1.4.3) mit einem entsprechend geringen Abstand $l_{\mathrm{s}}$ ist der Strömungswiderstandskoeffizient nochmals reduziert.

9 Der anfängliche Abstand der Tropfen untereinander steht in direktem Zusammenhang mit der dimensionslosen Wellenzahl bzw. der Wellenlänge der extern aufgeprägten Störung: $k a=\pi d / l_{\mathrm{s}} \Rightarrow l_{\mathrm{s}}=2 \pi / k=\lambda$. 


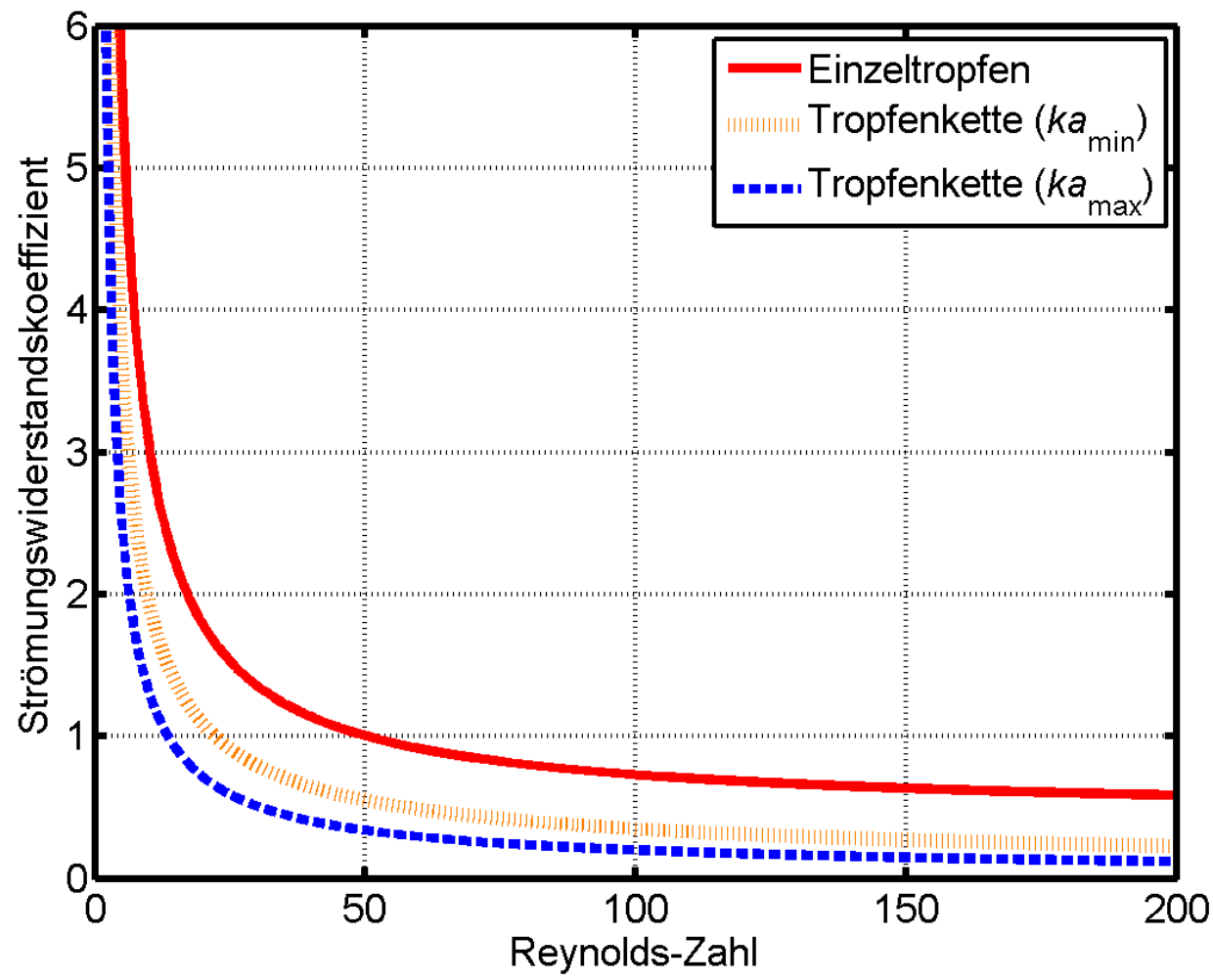

Abbildung 2.9: Strömungswiderstandskoeffizient als Funktion der Reynolds-Zahl. Gezeigt sind die Werte für den Einzeltropfen sowie für die größtmöglichen Tropfendurchmesser (entsprechend $k a_{\min }$ ) als auch die kleinstmöglichen Tropfendurchmesser (entsprechend $k a_{\max }$ ) in der Tropfenkette. Hierbei bestimmt die Tropfengröße den Abstand $l_{\mathrm{s}}$ zwischen den Tropfen in der Kette, der in den Strömungswiderstandskoeffizient eingeht.

In Abb. 2.10 sind die Lösungen der Modellgleichung (2.13) den experimentell bestimmten verzögerten Tropfengeschwindigkeiten in der Kette gegenübergestellt. Für drei verschiedene Ausgangsgeschwindigkeiten wurde aus den Hochgeschwindigkeitsaufnahmen der Seitenansicht die Tropfengeschwindigkeit innerhalb der Tropfenkette als Funktion des Abstands zur Düse bestimmt. Mit zunehmendem Abstand ist die erwartete Abbremsung zu beobachten. Bis etwa $20 \mathrm{~mm}$ unterhalb der Düse kann diese durch das Modell aus $|91|$ angenähert werden. Für größere Abstände zeigen sich jedoch Abweichungen. Diese Diskrepanz liegt vermutlich in der unzureichenden Genauigkeit von Gl. 2.14 begründet.

Im Weiteren (siehe Kap. 2.3.2 soll zusätzlich zur Beschreibung eines Tropfens innerhalb der Tropfenkette auch ein Tropfen in der Nähe der Kette beschrieben werden. Die Verwendung von Gl. 2.14 weist diesbezüglich insbesondere zwei Unzu- 


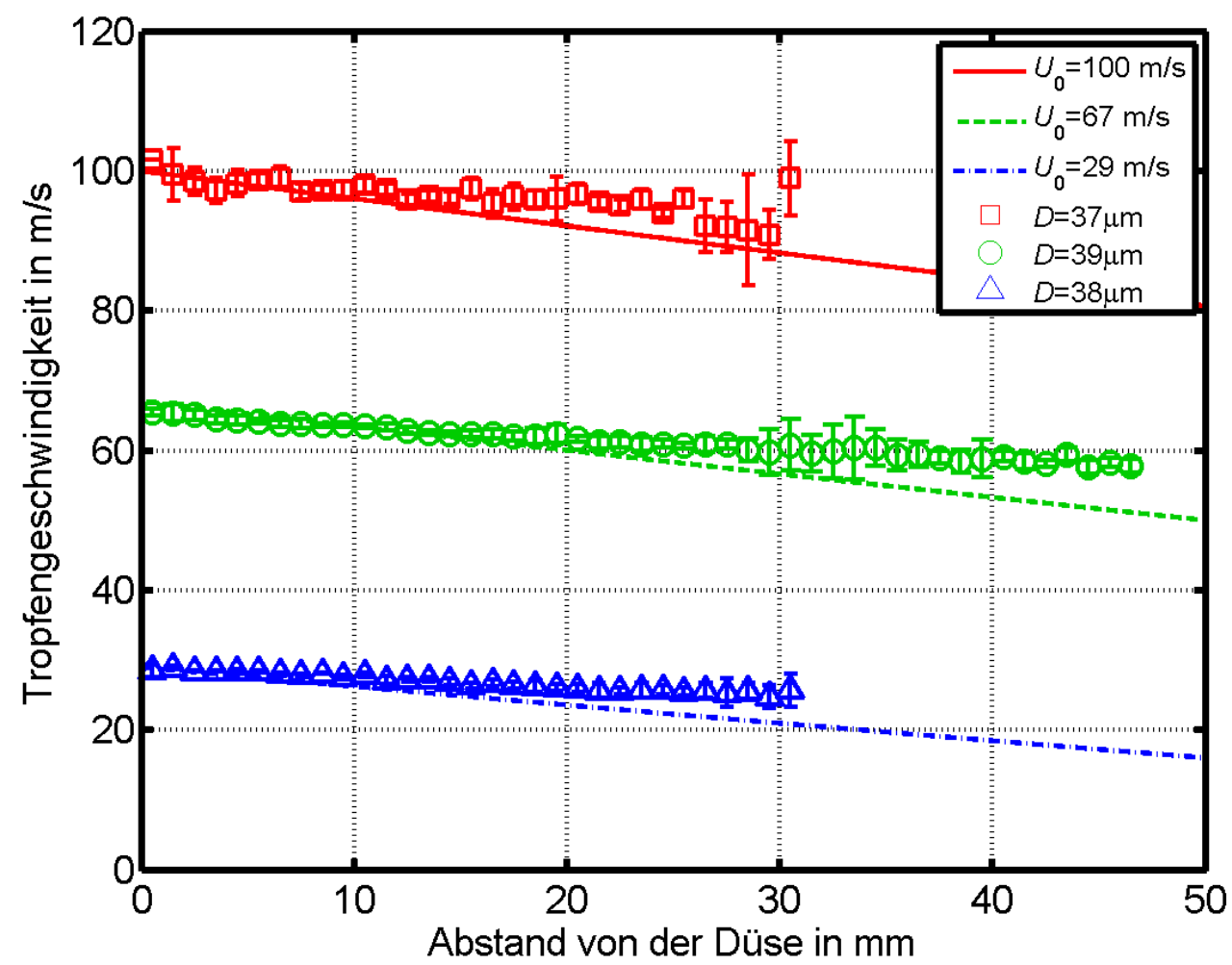

Abbildung 2.10: Tropfengeschwindigkeit innerhalb der Tropfenkette in Abhängigkeit des Abstands von der Düse. Für drei Austrittsgeschwindigkeiten $U_{0}$ ermöglicht die Annäherung in Gl. (2.14) (Linien) eine relativ gute Beschreibung der Abbremsung der Tropfen bis zu Abständen von etwa $20 \mathrm{~mm}$ unterhalb der Düse (Symbole: Messwerte). Danach wird die Verzögerung überschätzt.

länglichkeiten auf. Erstens ist der Strömungswiderstandskoeffizient während der Abbremsung der Tropfenkette in der Realität nicht konstant, da sich das Verhältnis $l_{\mathrm{s}} / D$ mit geringer werdender Geschwindigkeit verkleinert. Dieser Aspekt ließe sich jedoch durch eine iterative Berechnung der Abbremsung berücksichtigen 10 . Zweitens wird nur die Abbremsung eines Tropfens innerhalb der Tropfenkette beschrieben. Es wird also keine Aussage über die mitbewegte Luft getroffen. Dies führt in Summe zu einer Überschätzung der Reibungskraft.

Um eine genauere Beschreibung der Tropfentrajektorie innerhalb der Tropfenkette (und später auch nahe der Tropfenkette) zu ermöglichen, wird das Modell entsprechend den Untersuchungen von Lee [109] erweitert. Lee berücksichtigte den

10 In Abb. 2.10 wurde zur Verdeutlichung der Abweichung auf diese Anpassung verzichtet. Jedoch kommt es auch unter Berücksichtigung dieses Aspektes zu Abweichungen zwischen dem Modell und den experimentellen Ergebnissen. 


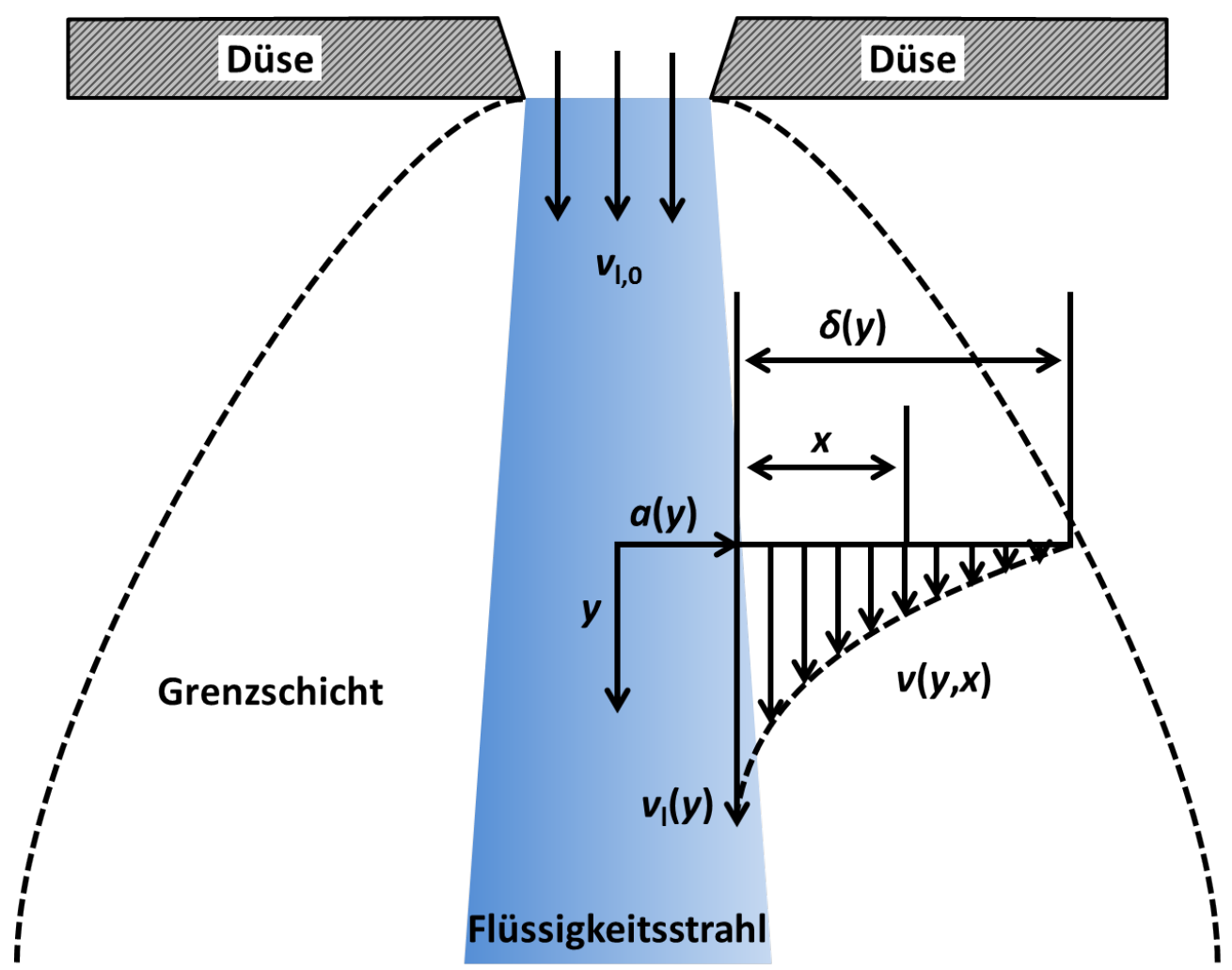

Abbildung 2.11: Schematische Darstellung der durch den Flüssigkeitsstrahl induzierten Ausbildung einer Grenzschicht $\delta(y)$ in der mitbewegten Luft (nicht maßstabsgetreu). Der Flüssigkeitsstrahl mit dem Radius $a(y)$ verlässt die Düse entlang der $\mathrm{y}$-Achse mit $v_{1,0}$. Es bildet sich ein Geschwindigkeitsfeld $v(y, x)$ in der umgebenden Luft. (Die Darstellung ist Abb. 1 aus [109] nachempfunden.)

Impulsübertrag der Tropfenkette auf die umgebende Luft. Dieser Impulsübertrag ist insbesondere variabel entlang der Tropfenkette, da diese mit zunehmendem Abstand zur Düse abgebremst wird. Als Resultat lässt sich nicht nur die Abbremsung der Tropfen, sondern auch das Geschwindigkeitsfeld in den angrenzenden Luftschichten berechnen (notwendig für die Berechnung der Tropfentrajektorie eines Einzeltropfens in der Nähe der Tropfenkette).

Im Folgenden wird entsprechend [109] angenommen, dass die Tropfenkette ab der Düse durch einen ununterbrochenen Flüssigkeitsstrahl mit einem Radius $a_{0}$ und einem ebenen Geschwindigkeitsprofil angenähert werden kann. Die verwendeten Bezeichnungen und Variablen sind in Abb. 2.11 dargestellt. Als weitere Grundlage dienen drei Gleichungen: 1) Massenerhaltung über den Strahlquerschnitt, 2) Impulserhaltung in axialer Richtung und 3) Ausgleich der Impulsabnahme der Flüssigkeit über die Viskosität der Luft. Zur Lösung der Grenzschichtgleichungen wird ein logarithmisches Profil der y-Komponente der Geschwindigkeit in der 
umgebenden Luft verwendet

$$
v(y, x)=v_{1}(y) \cdot\left(1-\frac{1}{\beta(y)} \ln \left(1+\frac{x}{a(y)}\right)\right)
$$

mit dem Faktor

$$
\beta(y)=\ln \left(1+\frac{\delta(y)}{a(y)}\right)
$$

Es werden die Randbedingungen erfüllt, dass die Luft am Rand der Grenzschicht in Ruhe ist und direkt am Flüssigkeitsstrahl die Strahlgeschwindigkeit $v_{1}(y)$ annimmt. Eine gewöhnlich zusätzliche Bedingung, dass die Änderung der Geschwindigkeit am Rand der Grenzschicht gleich Null ist, wird hingegen nicht erfüllt.

Die Geschwindigkeit der Flüssigkeit als Funktion des Abstands zur Düse lässt sich entsprechend [109] aus der Impulserhaltung in axialer Richtung unter Verwendung von Gl. 2.15) und durch Eliminierung von $a(y)$ mittels der Massenerhaltung berechnen. Es ergibt sich

$$
v_{1}^{\prime}(y)=\frac{v_{1}\left(y^{\prime}\right)}{v_{1,0}}=\frac{1}{1-\rho^{\prime}\left(1+\frac{1}{\beta}-\frac{1}{2 \beta^{2}}\left(\mathrm{e}^{2 \beta}-1\right)\right)},
$$

wobei gilt

$$
y^{\prime}=\frac{4 y}{a_{0} R e} \quad, \quad R e=\frac{2 a_{0} \rho_{\mathrm{g}} v_{1,0}}{\mu_{\mathrm{g}}} \quad \text { und } \quad \rho^{\prime}=\frac{\rho_{\mathrm{g}}}{\rho_{\mathrm{l}}} \quad .
$$

Mit der Massenerhaltung über den Strahlquerschnitt, dem Ausgleich der Impulsabnahme der Flüssigkeit über die Viskosität der Luft und Gl. 2.15) und (2.16) lässt sich finden

$$
\frac{\mathrm{d} \beta\left(y^{\prime}\right)}{\mathrm{d} y^{\prime}}=\frac{\beta^{2}-\rho^{\prime}\left(\beta^{2}+\beta-\frac{1}{2}\left(\mathrm{e}^{2 \beta}-1\right)\right)}{\beta\left(1+\mathrm{e}^{2 \beta}\right)-\left(\mathrm{e}^{2 \beta}-1\right)} .
$$

Zur Berechnung des gesamten Strömungsfelds (und der Flüssigkeitsgeschwindigkeit in Abhängigkeit vom Abstand von der Düse) muss $\beta$ als Funktion des normalisierten Abstands zur Düse $y^{\prime}$ integriert werden. Da am Düsenaustritt $\left(y^{\prime}=0\right)$ das Verhältnis der Dichten $\rho^{\prime}$ unbestimmt und die Grenzschichtdicke $\delta$ zunächst Null ist, ergibt sich in Gl. 2.17) eine Singularität bei numerischer Integration mit der Finite-Differenzen-Methode. Wie in [109 wird auch hier stattdessen $y^{\prime}$ als Funktion von $\beta$ mithilfe von MATLAB (MathWorks) integriert und dient als neuer Anfangswert zur Integration von Gl. (2.17). Bei der Integration ist darauf $\mathrm{zu}$ achten, dass ebenfalls $f(\beta)$ für sehr kleine $\beta \lesssim 10^{-5}$ bei der Berechnung numerische Unstetigkeiten aufweist. Abbildung 2.12 zeigt $y^{\prime}(\beta)=f(\beta)$. 


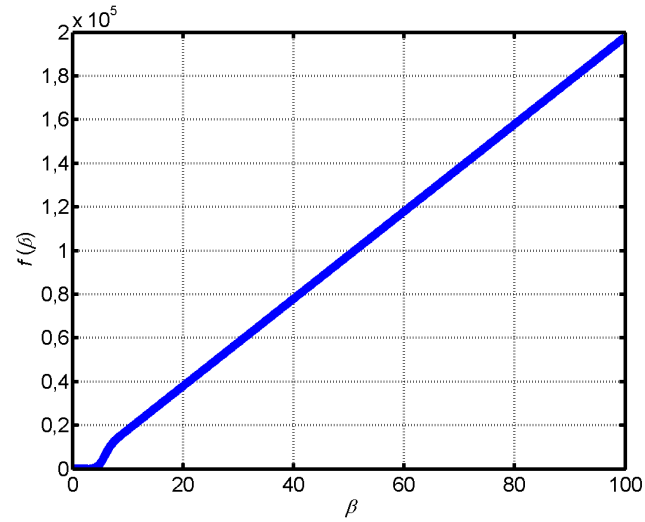

(a)

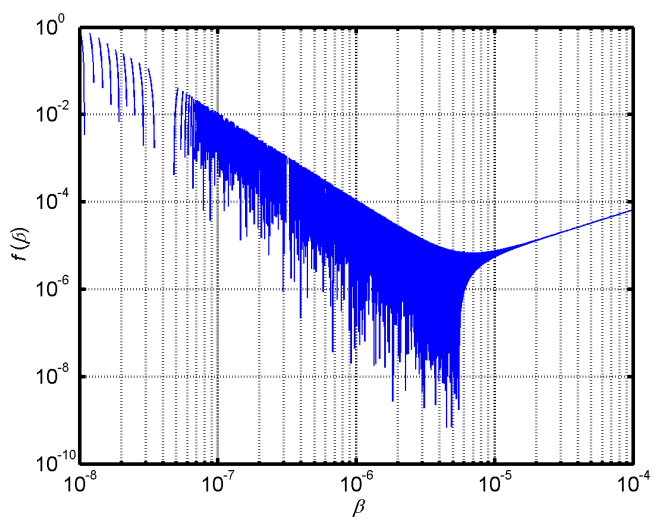

(b)

Abbildung 2.12: Die zu integrierende Funktion $f(\beta)$ ist (a) für große $\beta$ monoton steigend, weist jedoch aufgrund ihrer mathematischen Struktur (b) für kleine $\beta$ bei der Berechnung numerische Unstetigkeiten auf.

Abschließend wird das Verhältnis der Dichten $\rho^{\prime}$ angepasst. Da der Flüssigkeitsstrahl mit einem Durchmesser $d_{0}$ schon kurz unterhalb der Düse in Tropfen mit dem Durchmesser $D\left(>d_{0}\right)$ zerfällt, wird angenommen, dass dies näherungsweise direkt an der Düse geschieht. Nach einer Taylor-Entwicklung ergibt sich das angepasste Dichteverhältnis zu $\rho^{\prime}=\rho_{\mathrm{g}} D^{2} /\left(\rho_{\mathrm{l}} d_{0}^{2}\right)$.

Das normalisierte Geschwindigkeitsfeld im Gas ist in Abb. 2.13 dargestellt. Entlang der negativen $\mathrm{y}$-Achse (Position und Bewegungsrichtung der Tropfenkette) wird die Luft mit der Tropfengeschwindigkeit mitbewegt. In Abhängigkeit vom Abstand von der Düse $y^{\prime}$ wächst die Dicke $\delta$ der Grenzschicht, an deren Grenze keine Gasbewegung vorliegt $\left(v_{\mathrm{g}}^{\prime}=0\right)$. Für größere Abstände $x^{\prime}>\delta$ von der Tropfenkette setzt eine entgegengesetzt gerichtete Rückströmung der Luft ein 11 .

Ausgehend von Abb. 2.13 lässt sich mit Gl. 2.16) die zeitliche Entwicklung der Geschwindigkeit der Tropfenkette berechnen. In Abb. 2.14 wird dies für drei Austrittsgeschwindigkeiten und jeweils zwei Tropfendurchmesser mit den experimentellen Ergebnissen verglichen 12 , ähnlich zu der Auswertung in Abb. 2.10, In allen Fällen und über den gesamten Messbereich unterhalb der Düse zeigt sich eine sehr gute Übereinstimmung zum Modell von Lee [109] (im Gegensatz zum zuvor benutzten Modell von Kim et al. [91]).

11 Die Gültigkeit des Geschwindigkeitsfelds für große Abstände $x^{\prime}>\delta$ ist nicht unbedingt gewährleistet, aber auch nicht von großer Relevanz.

12 Die geringen Abweichungen im Bezug auf die drei Geschwindigkeiten sowie zwei Tropfendurchmesser ergeben sich aus der erst im Anschluss an das Experiment bestimmten tatsächlichen Geschwindigkeiten und Größen. 


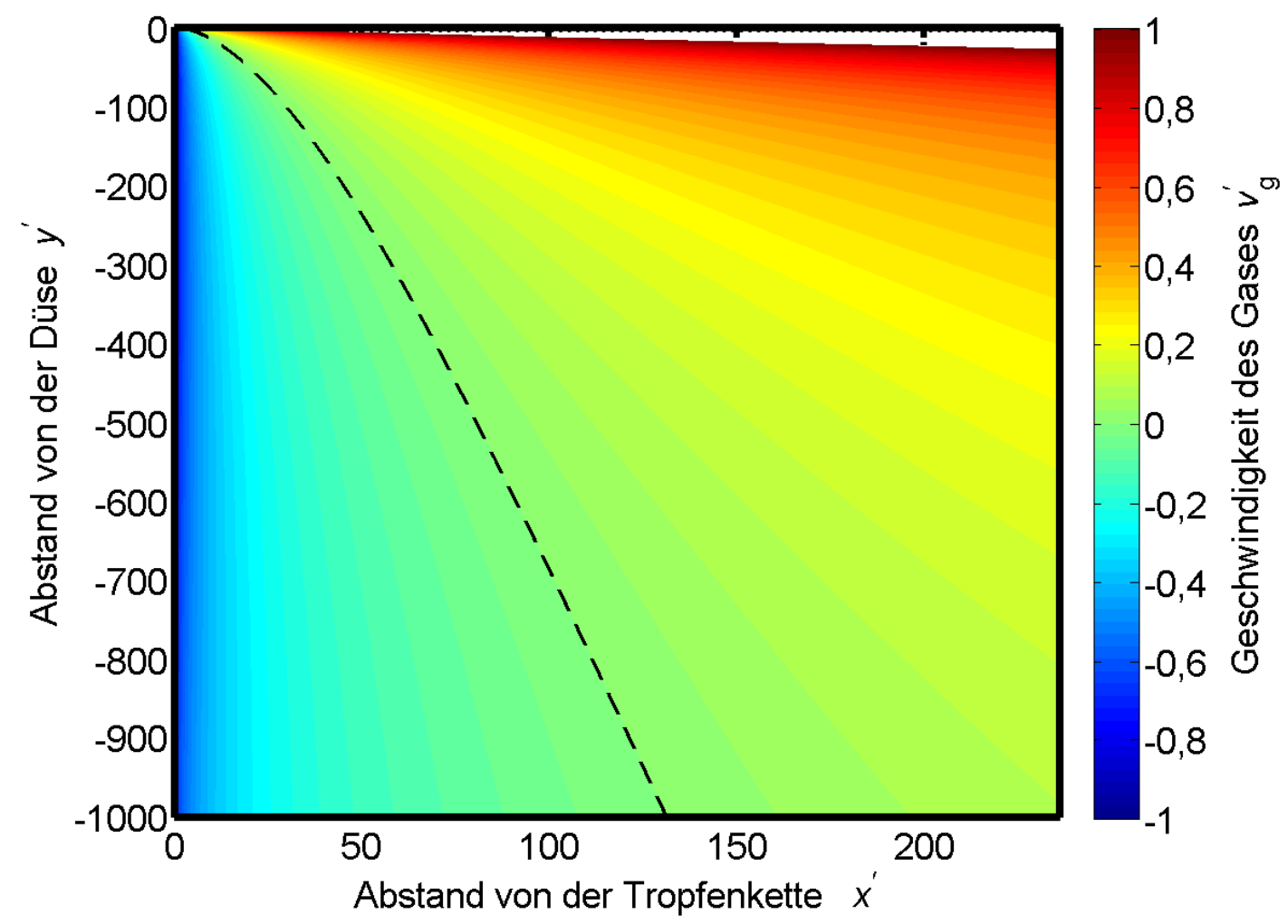

Abbildung 2.13: Normalisiertes Geschwindigkeitsfeld $v(x, y)$ in der die Tropfenkette umgebenden Luft. Die Tropfenkette verlässt die Düse bei $y^{\prime}=0$ in negativer y-Richtung. Direkt an der Tropfenkette $\left(x^{\prime}=0\right)$ strömt die Luft mit Tropfengeschwindigkeit $v_{\mathrm{g}}^{\prime}=-1$. Am Rand der Grenzschicht (gestrichelte Linie) ist die y-Komponente der Geschwindigkeit des Gases gleich Null. Außerhalb der Grenzschicht setzt eine in positive y-Richtung gerichtete Rückströmung ein.

Offensichtlich besteht nur ein geringer Unterschied in der Abbremsung für die unterschiedlichen Tropfendurchmesser, sowohl im Experiment als auch im Modell. Grund hierfür ist die Tatsache, dass die Tropfen jeweils aus dem gleichen Flüssigkeitsstrahl entstehen, womit der Impulsübertrag an die Luft identisch ist. Jedoch hat die unterschiedliche Tropfengröße einen Einfluss auf das Verhältnis der Dichten $\rho^{\prime}$. Zur Veranschaulichung ist in Abb. 2.15 dieser Sachverhalt dargestellt. Für die größtmögliche $(D=43 \mu \mathrm{m})$ und kleinstmögliche $(D=30 \mu \mathrm{m})$ Tropfengröße ist die Abnahme der Geschwindigkeit innerhalb der Tropfenkette gezeigt. Mit zunehmendem Abstand unterhalb der Düse wird deutlich, dass die kleineren Tropfen einer leicht geringeren Luftreibung ausgesetzt sind. Entgegengesetzt verhält es sich für einen Einzeltropfen. Entsprechend seines geringeren Impulses und höheren Strömungswiderstandskoeffizienten wird ein einzelner kleinerer 


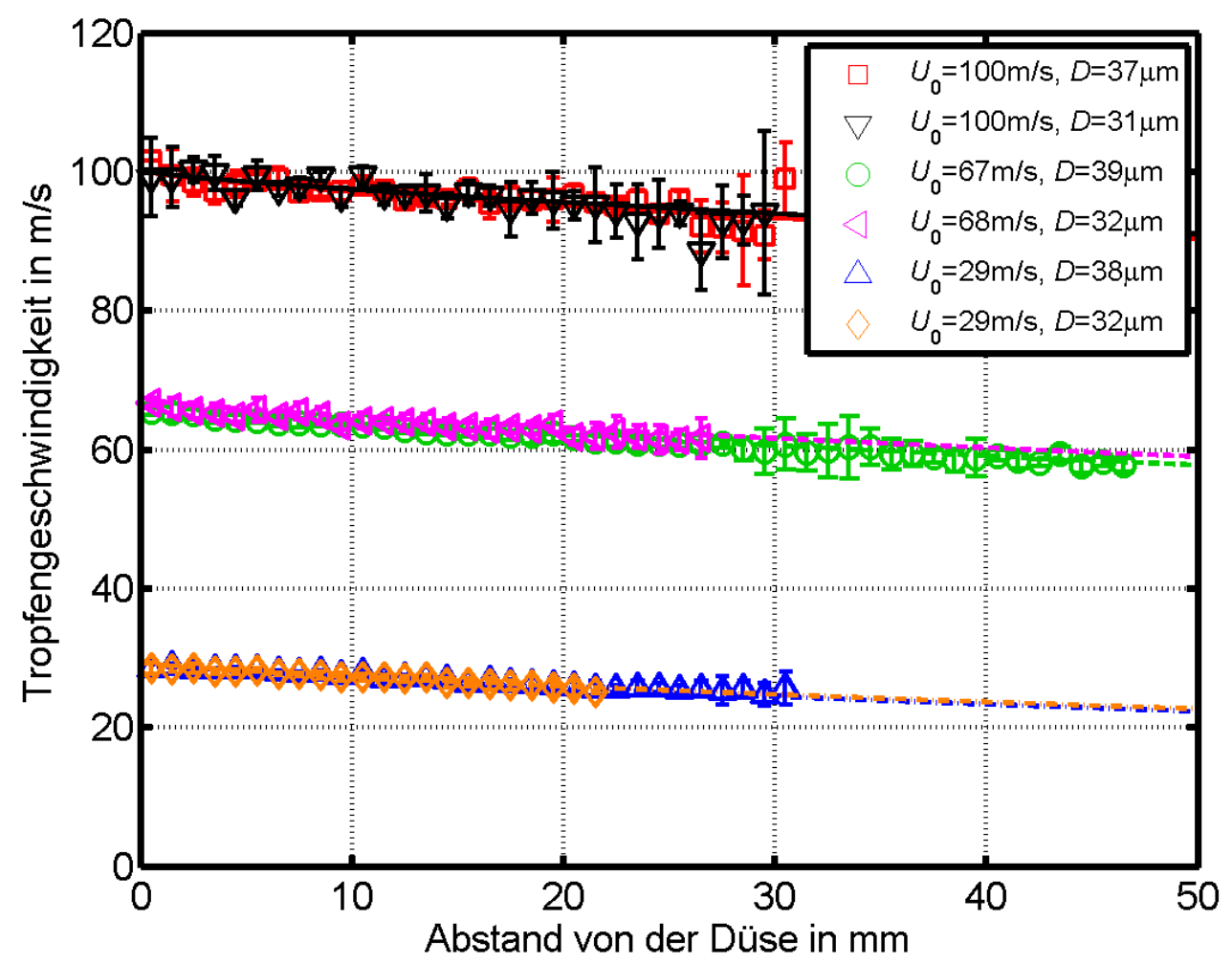

Abbildung 2.14: Tropfengeschwindigkeit innerhalb der Tropfenkette als Funktion des Abstands von der Düse. Für drei Austrittsgeschwindigkeiten $U_{0}$ und verschiedene Tropfendurchmesser $D$ ermöglicht Gl. 2.16) (Linien) eine gute Beschreibung der Abbremsung der Tropfen über den gesamten Messbereich unterhalb der Düse (Symbole: Messwerte).

Tropfen deutlich stärker abgebremst als ein größerer. In beiden Fällen ist jedoch eine deutlich stärkere Verlangsamung der Einzeltropfen gegenüber der Tropfenkette zu beobachten. Während die Geschwindigkeit im Falle der Tropfenkette nur um etwa $10 \%$ bei einem Abstand unterhalb der Düse von $50 \mathrm{~mm}$ abgefallen ist, so beträgt der Geschwindigkeitsverlust für Einzeltropfen etwa $45 \%$ bei den größtmöglichen Tropfen und etwa $65 \%$ bei den kleinstmöglichen Tropfen. 


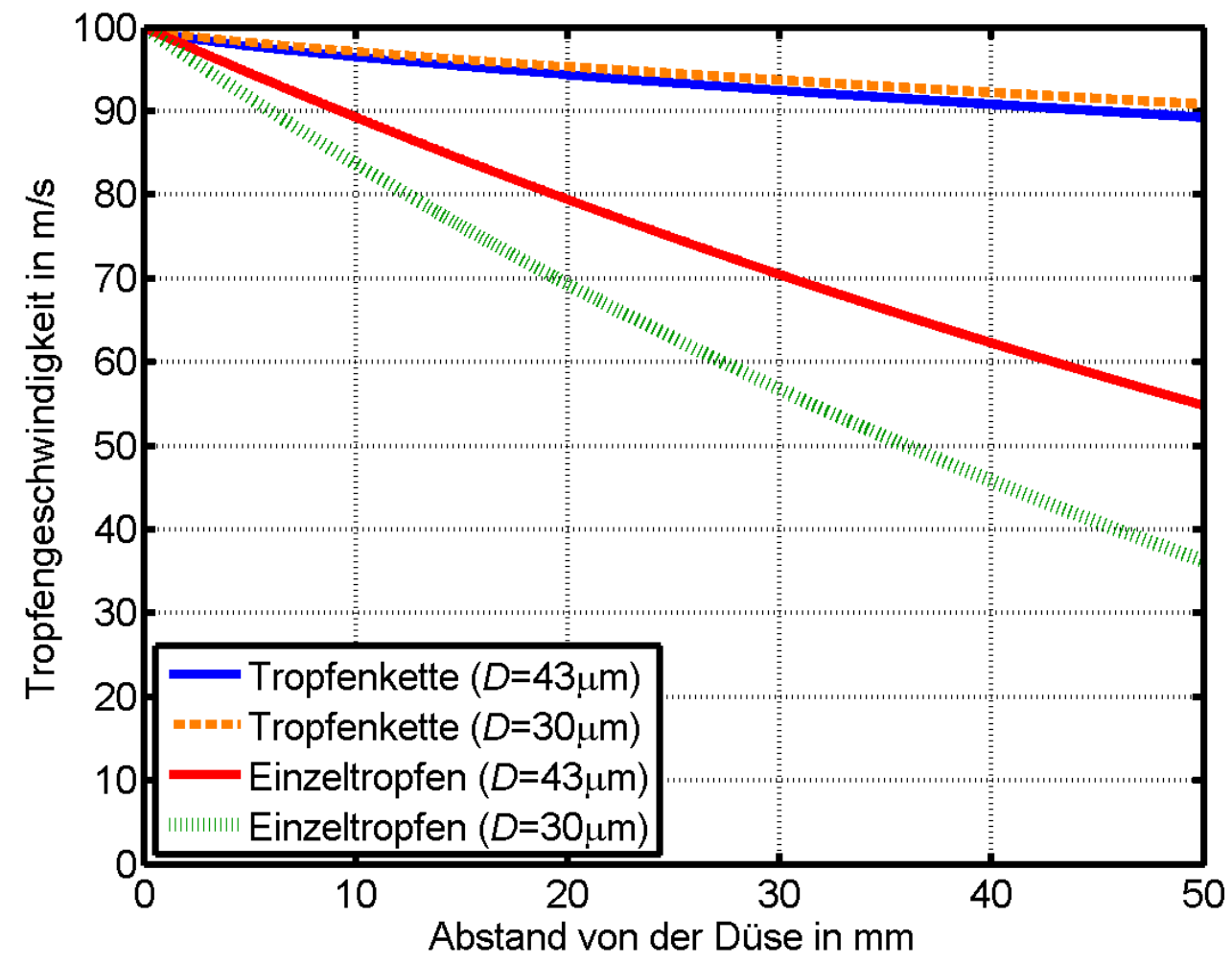

Abbildung 2.15: Tropfengeschwindigkeit innerhalb der Tropfenkette und für einen isolierten Einzeltropfen als Funktion des Abstands von der Düse. Es ist jeweils der größtmögliche $(D=43 \mu \mathrm{m})$ und kleinstmögliche $(D=30 \mu \mathrm{m})$ Tropfendurchmesser gezeigt. 


\section{Einzeltropfen in der Nähe der Tropfenkette}

Ausgehend von der Berechnung des Geschwindigkeitsfeldes in der die Tropfenkette umgebenden Luft wird dieses Modell auf einen einzelnen Tropfen in der Nähe der Tropfenkette übertragen.

Der Bewegung eines Einzeltropfens wirkt die Reibungskraft $\vec{F}_{\mathrm{D}}$ entsprechend Gl. (2.7) entgegen. Die zur Berechnung relevante Geschwindigkeit $U$ ist jedoch nicht bezogen auf die Düse, sondern relativ zur mitbewegten Luft. Die Geschwindigkeit der Luft als Funktion des Abstandes unterhalb der Düse und des Abstandes von der Tropfenkette ergibt sich nach Gl. 2.15). Entsprechend wirkt auf einen Tropfen innerhalb der Kette näherungsweise keine Reibungskraft, da die Luft sich mit der Geschwindigkeit der Tropfenkette bewegt. Außerhalb der Tropfenkette ist die Geschwindigkeit der mitbewegten Luft jedoch geringer und es kommt zu einer Abbremsung des Tropfens. Der Strömungswiderstandskoeffizient $C_{\mathrm{D} \text {, einzel }}$ entsprechend Gl. 2.8) wird ebenfalls mit dieser Relativgeschwindigkeit berechnet.

Abbildung 2.16a zeigt die Abbremsung eines Einzeltropfens mit einem Durchmesser von $37 \mu \mathrm{m}$ für drei verschiedene ursprüngliche Abstände $\Delta x$ zu der Tropfenkette (präpariert durch verschiedene Aufladungsdauern). Für jeden der drei Fälle wurde die Geschwindigkeit der mitbewegten Luft für den Abstand $\Delta x$ als Funktion des Abstands zur Düse berechnet und ging in die Lösung der Bewegungsgleichung ein. Es zeigt sich in allen Fällen über den Messbereich unterhalb des Düse eine relativ gute Übereinstimmung zwischen dem Modell und der experimentell bestimmten Änderung der Tropfengeschwindigkeit. Jedoch ist insbesondere für den Tropfen mit einem Abstand $\Delta x=230 \mu \mathrm{m}$ eine leichte Abweichung mit steigendem Abstand zur Düse sichtbar. Dieser Sachverhalt zeigt sich nochmals deutlicher in Abb. 2.16b mit einem erweiterten Messbereich für einen Tropfen mit einem ursprünglichen Abstand $\Delta x=80 \mu \mathrm{m}$ zur Kette. Die Beschreibung durch das Modell wird ab einem Abstand von etwa $30 \mathrm{~mm}$ unterhalb der Düse unzureichend. Offensichtlich lässt sich die Geschwindigkeitskomponente der Tropfenbewegung in $\mathrm{x}-$ Richtung (hervorgerufen durch das elektrische Ablenkungsfeld) nicht mehr vernachlässigen, die im vorliegenden Fall bis zu $1 \mathrm{~m} \mathrm{~s}^{-1}$ betrug. 


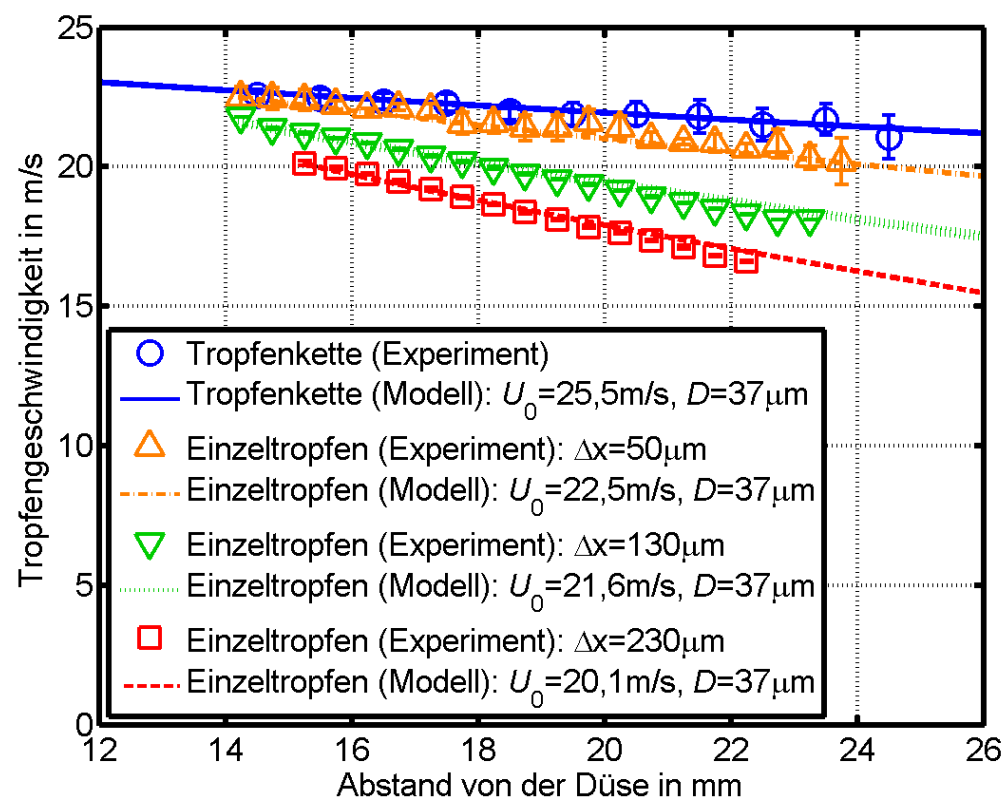

(a)

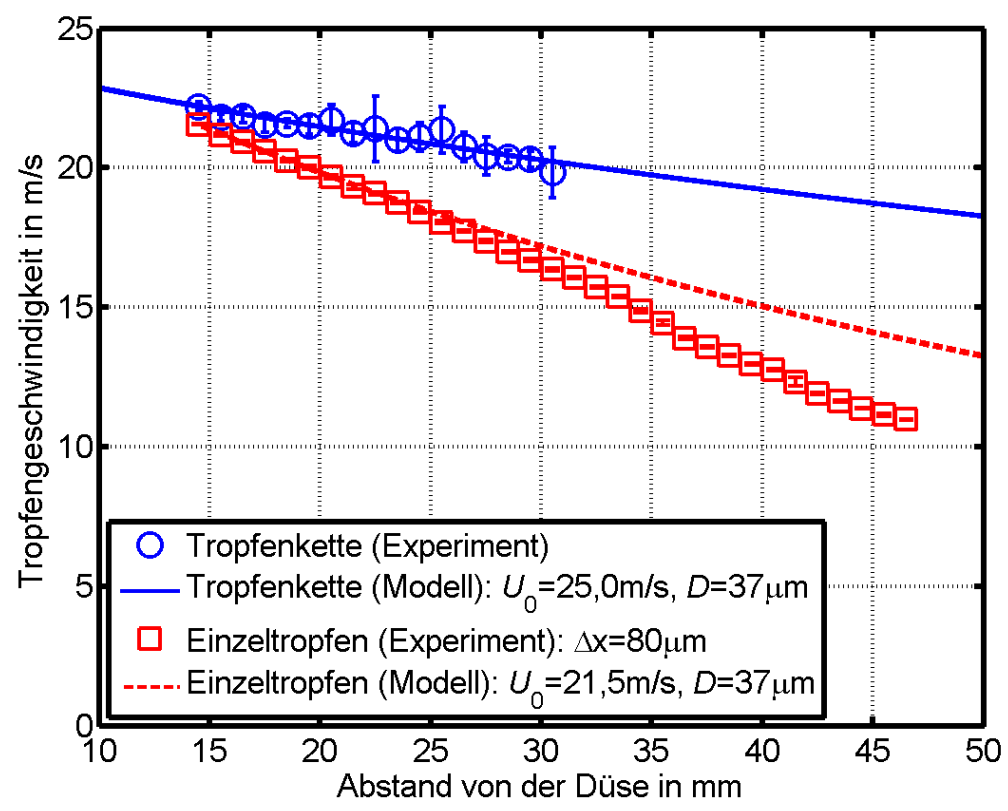

(b)

Abbildung 2.16: Abbremsung von Tropfen innerhalb und nahe der Tropfenkette als Funktion des Abstandes von der Düse (Symbole: Messwerte). (a): Die Berücksichtigung des Einflusses der mitbewegten Luft [109] ermöglicht eine gute Beschreibung der Abbremsung eines Einzeltropfens (gestrichelte Linien) sowohl innerhalb als auch nahe der Tropfenkette. (b): Für große Abstände unterhalb der Düse treten Abweichungen auf. 


\section{Vollständiges Modell der Tropfentrajektorie}

Die Berechnung des Geschwindigkeitsfelds der die Tropfenkette umgebenden Luft in Gl. 2.15) und die daraus resultierende Abbremsung eines Einzeltropfens in Gl. (2.7) werden in einem nächsten Schritt mit dem Einfluss des elektrischen Feldes in Gl.2.6) und der Gravitation in Gl. 2.5 verbunden. Des Weiteren werden der Prozess der Tropfenaufladung in den Gleichungen (2.1) und 2.3 und die Grundlagen der Erzeugung einer monodispersen Tropfenkette entsprechend Kap. 1 mit einbezogen. Somit lässt sich die in Gl. (2.11) formulierte Differentialgleichung unter Berücksichtigung des gesamten Prozesses von Erzeugung über Aufladung und bis hin zur Ablenkung eines Einzeltropfens lösen.

In Abb. 2.17 ist der Vergleich zwischen dem (nun vollständigen) Modell und der experimentell bestimmten Trajektorie eines Einzeltropfen dargestellt. Hierfür wurden die Messergebnisse aus Abb. 2.5 genutzt. Tabelle 2.1 führt die einzelnen Parameter auf und dient zur weiteren Erläuterung von Abb. 2.17. Das elektrische Ablenkfeld wurde mittels Finite Elemente Simulationen (Electrostatics, COMSOL) berechnet. Die Tropfengeschwindigkeit in $\mathrm{y}$-Richtung nimmt durch die Luftreibung stetig ab (siehe Abb. 2.17a). Der Tropfen erfährt durch das elektrische Feld zunächst eine positive Beschleunigung in $\mathrm{x}$-Richtung. Diese wird nach etwa 0,6 ms (beim Austritt aus den Ablenkungselektroden) durch die Luftreibung überkompensiert und es kommt ebenfalls zu einer stetigen Abnahme der Geschwindigkeit. Mittels der zeitlichen Änderung der Tropfengeschwindigkeit in $\mathrm{x}-$ und $\mathrm{y}$-Richtung lässt sich die theoretische Trajektorie mit der experimentell bestimmten vergleichen. Es zeigt sich eine sehr gute Übereinstimmung zwischen dem Modell und dem Experiment.

Bei der Versuchsdurchführung konnten nahezu alle notwendigen Parameter, wie in Tab. 2.1 gegeben, eingestellt bzw. gemessen werden. Lediglich die Zeitkonstante der Aufladung $\tau$ und die maximal übertragbare Ladungsmenge $Q_{\max }$ konnten nur indirekt gemessen werden. Aus einem Vergleich mit den Ergebnissen in Abb. 2.5 lässt sich $\tau$ jedoch als 2,8 us entnehmen. Die gleichzeitige Verwendung von $Q_{\max }$ aus derselben Abbildung würde jedoch zu einer zu geringen übertragenen Ladungsmenge führen, und die theoretische und experimentelle Trajektorie würden damit stark voneinander abweichen. Daher wurde in der Berechnung eine angenäherte maximale Ladungsmenge von $Q_{\max }^{\prime}=1,77 \times 10^{-13} \mathrm{C}$ verwendet. Diese a posteriori Festlegung wird wie folgt begründet: Sowohl der Aufladungsprozess als auch die Tropfenabbremsung lassen sich für sich betrachtet sehr gut mit den einzelnen Modellen beschreiben (siehe Kap. 2.3.1 und Kap. 2.3.2. Daher kann von ihrer hinreichenden Genauigkeit ausgegangen werden. Bei Zusammensetzung beider Teilaspekte wäre nun eine ebenso gute Übereinstimmung zu 
Tabelle 2.1: Verwendete Parameter zur Lösung der Differentialgleichung 2.15 der Tropfentrajektorie.

\begin{tabular}{lll} 
Parameter & Symbol & Einheit \\
\hline anfängliche x-Position & $X_{0}$ & $0 \mathrm{~m}$ \\
anfängliche y-Position & $Y_{0}$ & $0 \mathrm{~m}$ \\
anfängliche horizontale Geschwindigkeit & $U_{x, 0}$ & $0 \mathrm{~m} \mathrm{~s}^{-1}$ \\
anfängliche vertikale Geschwindigkeit & $U_{y, 0}$ & $-26,2 \mathrm{~m} \mathrm{~s}^{-1}$ \\
Düsendurchmesser & $d$ & $16,8 \mu \mathrm{m}$ \\
akustische Anregungsfrequenz & $f$ & $210 \mathrm{kHz}$ \\
Tropfendurchmesser & $D$ & $37,5 \mu \mathrm{m}$ \\
Aufladungsspannung & $U_{\text {aufl }}$ & $300 \mathrm{~V}$ \\
Radius der Aufladungselektrode & $R_{\text {aufl }}$ & $0,5 \mathrm{~mm}$ \\
Zeitkonstante der Aufladung & $\tau$ & $2,8 \mu \mathrm{s}$ \\
maximale Ladung auf dem Tropfen & $Q_{\text {max }}^{\prime}$ & $1,77 \times 10^{-13} \mathrm{C}$ \\
Ablenkungsspannung & $U_{\text {abl }}$ & $1,2 \mathrm{kV}$ \\
Abstand der Ablenkungselektroden & $d_{\text {abl }}$ & $3 \mathrm{~mm}$ \\
Länge der Ablenkungselektroden & $l_{\text {abl }}$ & $10 \mathrm{~mm}$ \\
\hline
\end{tabular}

erwarten. Es konnte jedoch schon in Abb. 2.7 gezeigt werden, dass eine Abweichung in Form eines Vorfaktors zwischen der experimentell bestimmten und der durch das Modell von Schneider et al. 82 vorhergesagten maximalen Ladungsmenge existiert. Dies ist vermutlich zum einen auf die Annahme eines räumlich konstanten elektrischen Feldes zur Ablenkung (siehe Gl. (2.4)) als auch auf die Tatsache einer nicht zylinderförmigen, sondern irisförmigen Aufladungselektrode (siehe Abb. 2.2), zurückzuführen. Ausgehend von der Richtigkeit der weiteren Annahmen, verbleibt nur $Q_{\max }$ als anzupassende Größe. Die Gültigkeit dieser Behauptung wird ebenfalls durch die Tatsache bekräftigt, dass die experimentelle Krümmung der Tropfentrajektorie in Abb. 2.17 mit dem Modell übereinstimmt. 


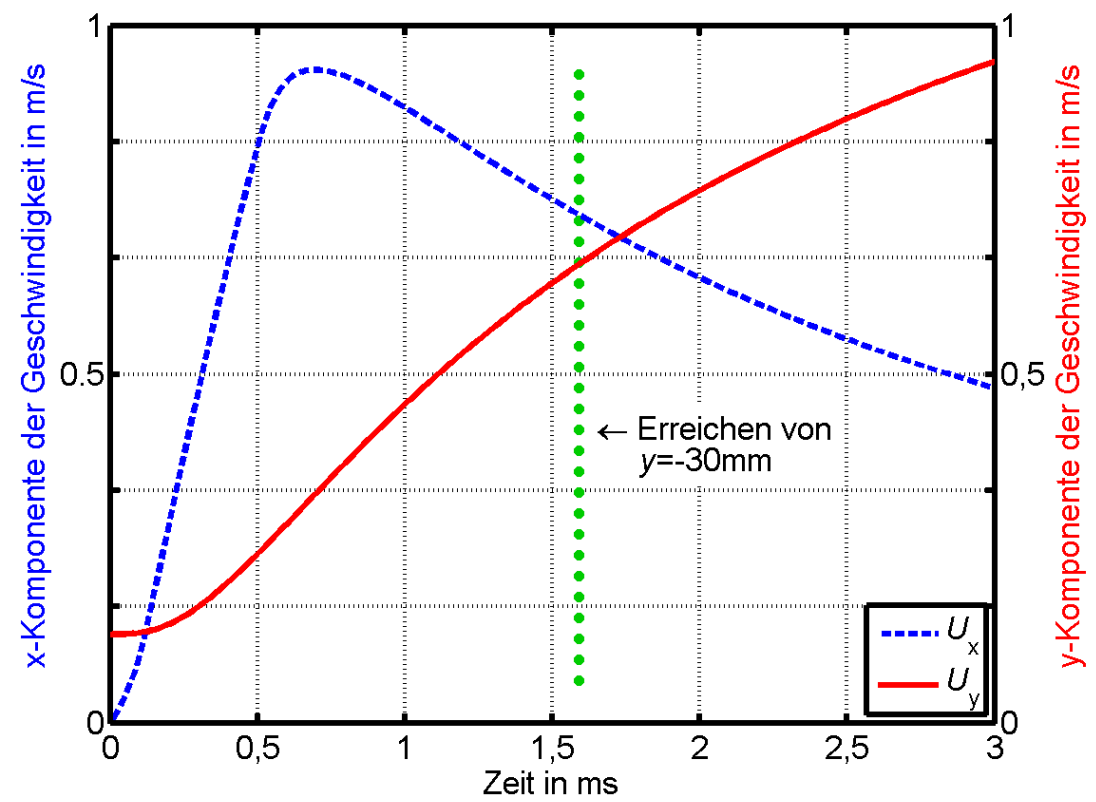

(a)

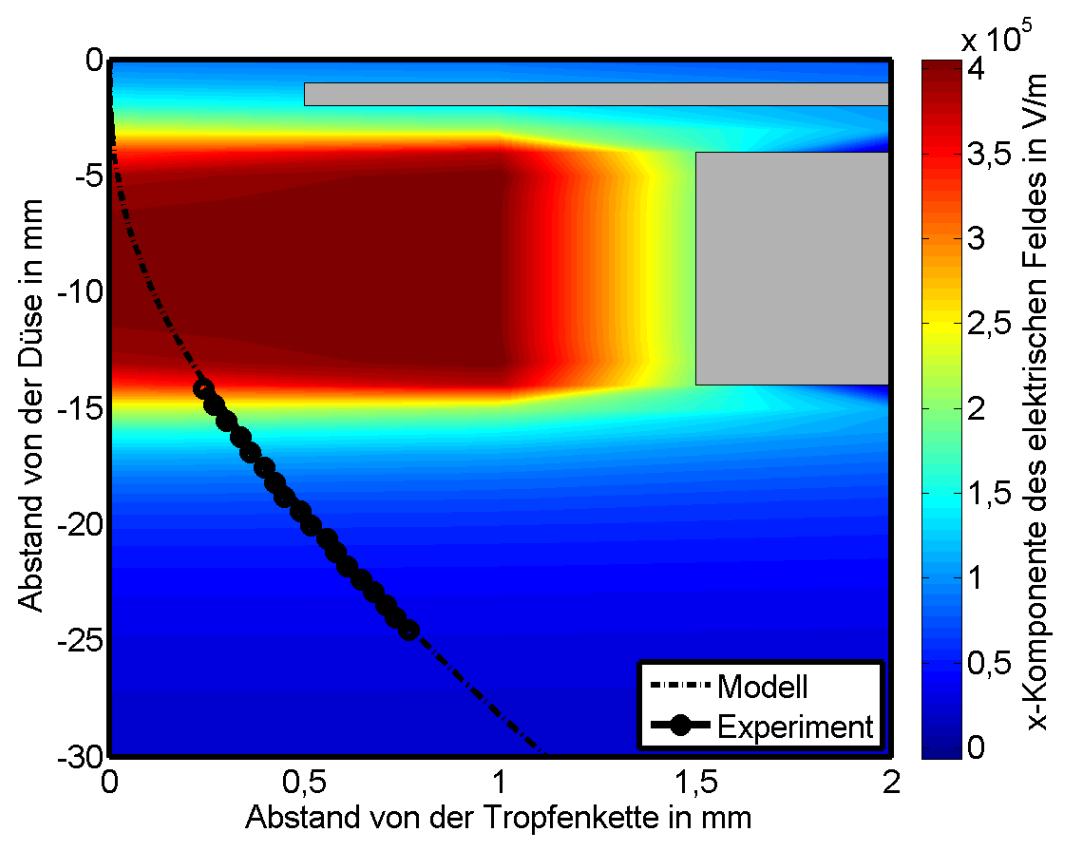

(b)

Abbildung 2.17: Berechnung der Trajektorie eines Einzeltropfens (Parameter sind in Tab. 2.1 gegeben). (a): Zeitliche Änderung der Tropfengeschwindigkeit in $\mathrm{x}$-Richtung (gestrichelte Linie) und y-Richtung (durchgezogene Linie) entsprechend Gl. 2.11). Erreichen des in (b) gezeigten Ausschnitts bis $y=-30 \mathrm{~mm}$ (gepunktete Linie). (b): Der Vergleich zwischen der experimentell bestimmten (Symbole) und mit dem Modell berechneten (gestrichelte Linie) Tropfentrajektorie weist eine gute Übereinstimmung auf. Die lokale Stärke des elektrischen Ablenkfeldes in $\mathrm{x}$-Richtung ist farblich dargestellt. Die grauen Flächen deuten Position und Geometrie der Aufladungs- bzw. Ablenkungselektroden an. 


\subsubsection{Limitierung der Methode}

Unter der Annahme, dass sich mit den Aufladungs- und Ablenkungsspannungen die Einzeltropfen immer weit genug herauslenken lassen, erfolgt nun eine Abschätzung über die erreichbaren maximalen Einschlagsgeschwindigkeiten.

Für die Durchführung eines Experiments zum Einschlag von Einzeltropfen ist es notwendig, den Tropfen um einen Abstand $\Delta x>x_{\min }=D+100 \mu \mathrm{m}$ aus der Tropfenkette herauszulenken (siehe Kap. 2.2.1) und die Tropfenkette in eine Auffangvorrichtung umzuleiten. Die Geometrie der Auffangvorrichtung erfordert im Experiment einen zusätzlichen vertikalen Abstand von $\Delta y=5 \mathrm{~mm}$ bis der Tropfen auf einem Substrat auftreffen kann. Ab einer Position, an der die Tropfenkette vom Einzeltropfen getrennt ist und die Tropfenkette in die Auffangvorrichtung umgeleitet wird, wird angenommen, dass das Geschwindigkeitsfeld der mitbewegten Luft stark abnimmt und vernachlässigbar ist.

Abbildung 2.18 zeigt für verschiedene anfängliche Tropfengeschwindigkeiten $U_{y, 0}$ beim Austritt aus der Düse die theoretisch erreichbaren Einschlagsgeschwindigkeiten $U_{\max }$ auf dem Substrat. Die zur Berechnung verwendeten Parameter sind identisch zu den zuvor in Tab. 2.1 angegebenen. Durch Verwendung einer konstanten dimensionslosen Wellenzahl $k a$ ist die Tropfengröße für alle Geschwindigkeiten konstant. Die übertragene Ladung $Q$ ist dabei von der Dauer der akustischen Anregungsperiode $T=1 / f=\pi d /\left(k a U_{y, 0}\right)$ abhängig.

Es zeigt sich, dass die erhöhte Luftreibung nach Herauslenkung des Einzeltropfens aus der Tropfenkette die erreichbare Einschlagsgeschwindigkeit deutlich reduziert. So wird z.B. die maximal erreichbare Einschlagsgeschwindigkeit von etwa $45 \mathrm{~m} \mathrm{~s}^{-1}$ für eine Austrittsgeschwindigkeit von etwa $80 \mathrm{~m} \mathrm{~s}^{-1}$ erreicht. Nach diesem Maximum nimmt bei noch höheren Austrittsgeschwindigkeiten die erreichbare Einschlagsgeschwindigkeit wieder ab. Dies ist dadurch begründet, dass mit steigender Tropfengeschwindigkeit die übertragene Ladungsmenge abnimmt. Entsprechend wird die nötige Ablenkung $\Delta x>x_{\text {min }}$ erst bei größerem Abstand von der Düse erreicht, was wiederum eine nochmals geringere Geschwindigkeit zur Folge hat.

Ein Vergleich (siehe Abb. 2.18b mit dem notwendigen Abstand des Substrates, der sich aus der Position des Erreichens der zu erzielenden Einschlagsgeschwindigkeit ergibt, zeigt ein monotones Wachstum mit steigender Austrittsgeschwindigkeit. Die maximal erreichbare Einschlagsgeschwindigkeit von etwa $45 \mathrm{~m} \mathrm{~s}^{-1}$ lässt sich nur für einen Abstand des Substrates von etwa $80 \mathrm{~mm}$ unterhalb der Düse erreichen. Ein dichterer Abstand ist durch die noch nicht ausreichende Tropfenablenkung und ein weiterer Abstand durch die Tropfenabbremsung nicht erreichbar. 


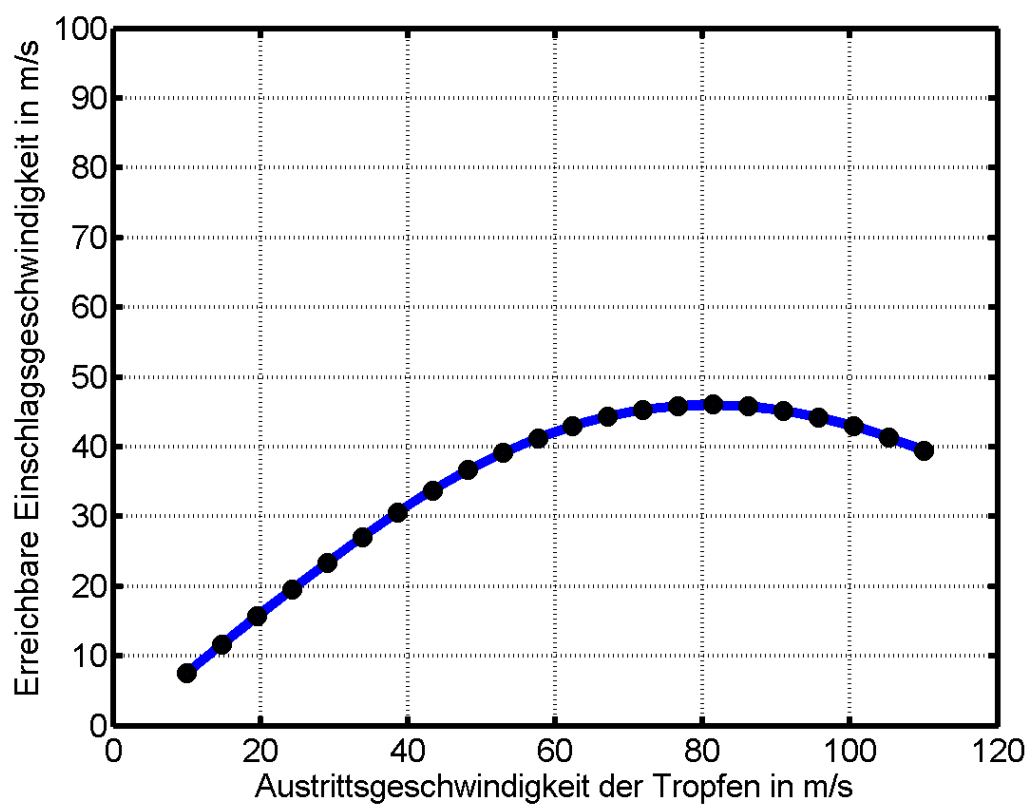

(a)

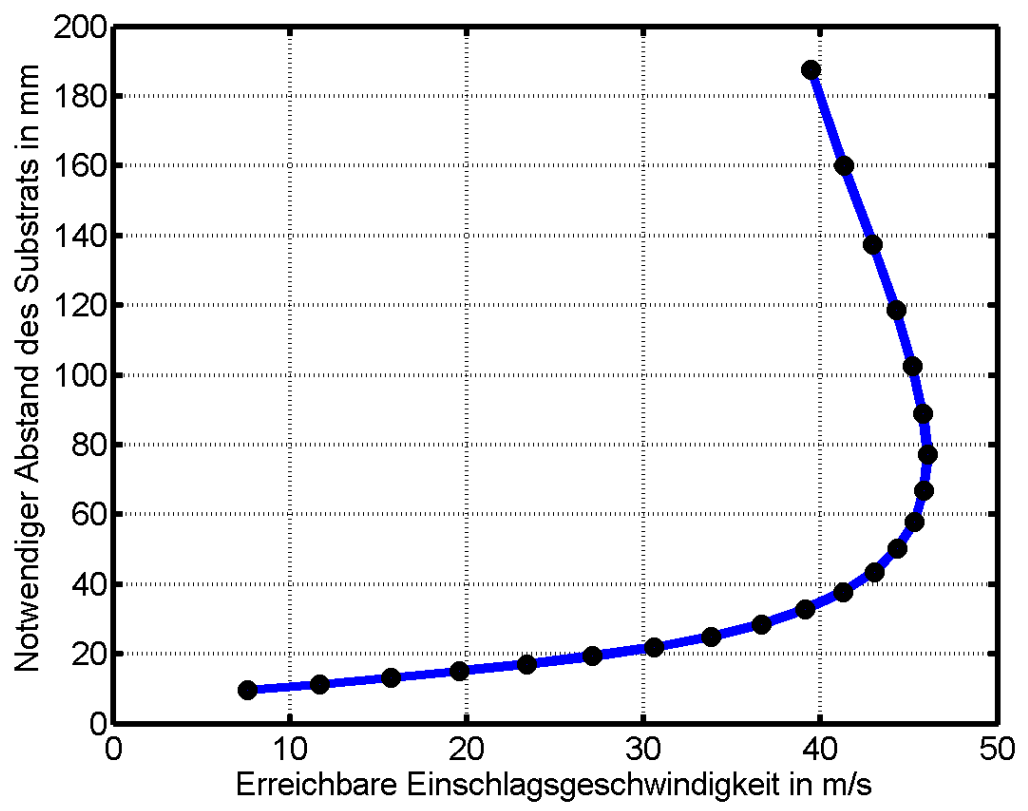

(b)

Abbildung 2.18: (a): Maximal erreichbare Einschlagsgeschwindigkeiten für einen Tropfen von 37,5 $\mathrm{m}$ Durchmesser als Funktion der Austrittsgeschwindigkeit an der Düse. Es kommt nach der Herauslenkung aus der Tropfenkette zu einer starken Abbremsung aufgrund der Luftreibung. Die maximal erreichbare Einschlagsgeschwindigkeit von etwa $45 \mathrm{~m} \mathrm{~s}^{-1}$ wird für eine Austrittsgeschwindigkeit von etwa $80 \mathrm{~m} \mathrm{~s}^{-1}$ erreicht. (b): Abhängig von der Austrittsgeschwindigkeit verändert sich die Position unterhalb der Düse, ab der eine ausreichende Ablenkung $\Delta x>x_{\min }$ erreicht wird. 
Eine weitere Steigerung ${ }^{13}$ der erreichbaren Einschlagsgeschwindigkeit ist möglich, indem sowohl die Aufladungsspannung auf $U_{\text {auf }}=1 \mathrm{kV}$ als auch die Stärke des elektrischen Feldes zur Tropfenablenkung auf $E_{x, \text { abl }}=1 \mathrm{MV} \mathrm{m}^{-1}$ erhöht werden. Außerdem kann die Länge der Ablenkungselektroden $l_{\mathrm{abl}}$ so erweitert werden, dass eine konstante Feldstärke über die gesamte Tropfenablenkung gewährleistet ist. Dies wurde nur in der nachfolgenden Berechnung, jedoch nicht im Experiment umgesetzt. Die Ergebnisse der Berechnung für ansonsten unveränderte Parameter sind in Abb. 2.19 dargestellt. Es wird deutlich, dass durch diese Anpassung höhere Einschlagsgeschwindigkeiten bei gleichzeitig geringeren notwendigen Abständen des Substrats erreicht werden können. Im Vergleich zur zuvor erreichbaren maximalen Einschlagsgeschwindigkeit von etwa $45 \mathrm{~m} \mathrm{~s}^{-1}$ bei einer Austrittsgeschwindigkeit von etwa $80 \mathrm{~m} \mathrm{~s}^{-1}$ lassen sich nun etwa $60 \mathrm{~m} \mathrm{~s}^{-1}$ erzielen. Des Weiteren ist im berechneten Bereich bis $110 \mathrm{~m} \mathrm{~s}^{-1}$ Austrittsgeschwindigkeit kein Erreichen einer maximalen Einschlagsgeschwindigkeit zu beobachten. Sie wird vielmehr durch die experimentell mögliche Austrittsgeschwindigkeit der Tropfen beschränkt (bzw. hier in der Berechnung auf $110 \mathrm{~m} \mathrm{~s}^{-1}$ gesetzt).

Die zuvor gemachte Annahme eines räumlich konstanten elektrischen Feldes zur Tropfenablenkung ließ sich experimentell nicht umsetzen; daher soll im nächsten Schritt wieder die im Experiment verwendete Länge $l_{\mathrm{abl}}=10 \mathrm{~mm}$ genutzt werden. Ausgehend von dem zusammengefügten Modell in Kap. 2.3.2 wird die erreichbare Einschlagsgeschwindigkeit auf weitere Tropfendurchmesser erweitert. Es wurden hierzu folgende Annahmen gemacht:

I) Der Prozess zur Erzeugung einer monodispersen Tropfenkette ist im Experiment für eine dimensionslose Wellenzahl $k a=0,69$ am stabilsten (siehe Kap. 1.2.2. Es wird daher angenommen, dass Düsen mit entsprechendem Durchmesser $d$ zur Erzeugung eines bestimmten Tropfendurchmessers $D$ verwendet werden.

II) Die Ergebnisse in Abb. 2.6 zeigen eine Schwankung der Zeitkonstante der Aufladung $\tau$. Daher wird sie entsprechend Gl. (2.3) angenähert und weist eine direkte Abhängigkeit von dem Durchmesser der Düse bzw. des Flüssigkeitsstrahls auf.

III) Ausgehend von den Ergebnissen in Abb. 2.7 liegt die experimentell bestimmte maximale Ladung unter der theoretisch erwarteten entsprechend Gl. 2.1). Dies ist durch die Vereinfachungen im Modell begründet. Die maximale Ladung wird daher mit $Q_{\max }^{\prime}=0,2 \cdot Q_{\max }$ berechnet.

13 Eine derartige Umsetzung im Experiment erfordert einen entsprechenden Aufwand in der Isolation des Aufbaus, um dem steigenden Risiko elektrischer Entladungen entgegenzuwirken. 


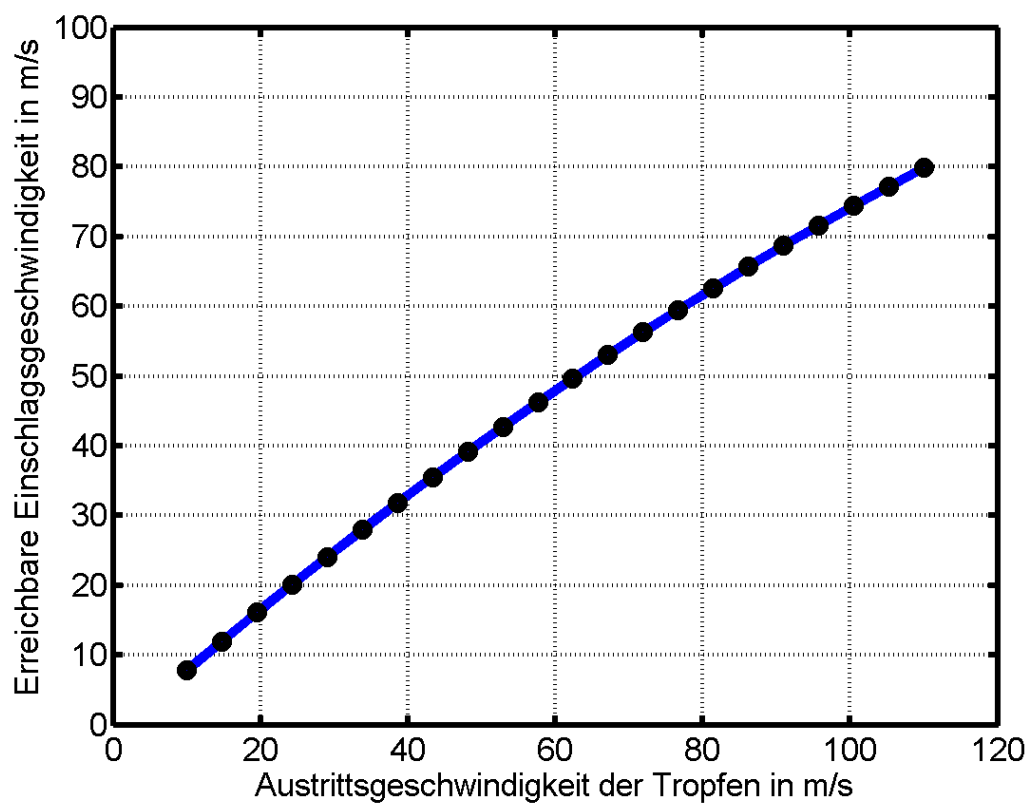

(a)

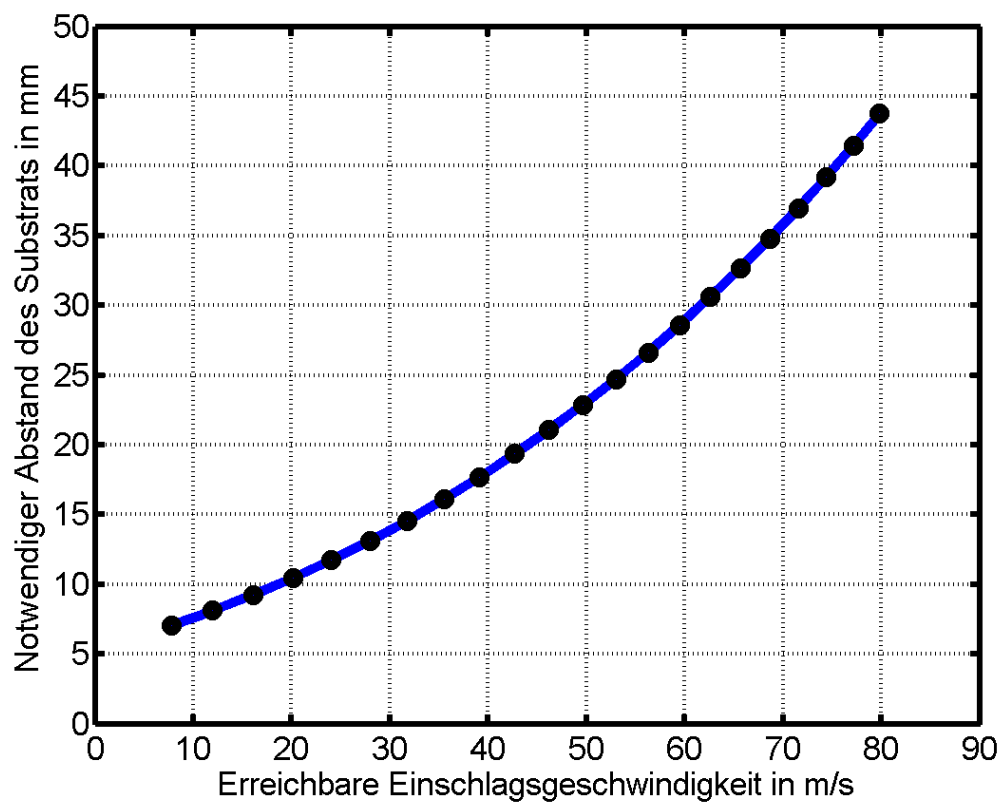

(b)

Abbildung 2.19: (a): Maximal erreichbare Einschlagsgeschwindigkeiten für einen

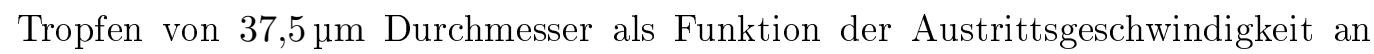
der Düse. Es kommt nach der Herauslenkung aus der Tropfenkette zu einer starken Abbremsung aufgrund der Luftreibung. Die maximal erreichbare Einschlagsgeschwindigkeit weist kein Maximum auf. (b): Abhängig von der Austrittsgeschwindigkeit verändert sich die Position unterhalb der Düse, ab der eine ausreichende Ablenkung $\Delta x>x_{\min }$ erreicht wird. 
In Abb. 2.20 ist die erreichbare Einschlagsgeschwindigkeit für Tropfendurchmesser von $10 \mu \mathrm{m}$ bis $100 \mu \mathrm{m}$ und Austrittsgeschwindigkeiten von $10 \mathrm{~m} \mathrm{~s}^{-1}$ bis $120 \mathrm{~m} \mathrm{~s}^{-1}$ zu sehen. Auch mit der ursprünglichen Länge der Ablenkungselektroden von $10 \mathrm{~mm}$ ist noch kein Maximum der Einschlagsgeschwindigkeit zu sehen (siehe Abb. 2.19). Durch die Erhöhung der Aufladungs- und Ablenkungsspannung ist die erreichbare Einschlagsgeschwindigkeit im betrachteten Bereich von der experimentell möglichen Austrittsgeschwindigkeit $\left(120 \mathrm{~m} \mathrm{~s}^{-1}\right)$ der Tropfen begrenzt. Es kommt jedoch in allen Fällen zu einer sichtbaren Abbremsung der Tropfen, sodass alle Datenwerte stets unterhalb der Gerade $U_{\max }=U_{y, 0}$ (gestrichelte Linie) liegen.

Es zeigt sich, dass die maximal erreichbare Einschlagsgeschwindigkeit nicht monoton mit dem Tropfendurchmesser ansteigt, sondern im Bereich von $60 \mu \mathrm{m}$ ein Maximum von etwa $85 \mathrm{~m} \mathrm{~s}^{-1}$ aufweist. Für kleine Tropfen mit $D \lesssim 15 \mu \mathrm{m}$ lassen sich nur deutlich geringere Einschlagsgeschwindigkeiten erreichen. Dies ist durch eine geringere übertragene Ladungsmenge begründet. Sowohl kleinere Durchmesser des Flüssigkeitsstrahls (siehe Gl.(2.3p) als auch die kürzere akustische Periode der Aufladung (siehe Gl. (2.2)) führen zu einer geringeren übertragenen Ladungsmenge. Entsprechend wird eine ausreichende Herauslenkung des Einzeltropfens erst für größere Abstände unterhalb der Düse erreicht, und daher ist die resultierende Einschlagsgeschwindigkeit geringer. Außerdem ist die Abbremsung bei abnehmender Tropfengröße deutlich stärker, wie es auch in Abb.2.15zu sehen ist ${ }^{14}$. Diese Tatsache wird am Beispiel von Tropfen mit $D=10 \mu \mathrm{m}$ deutlich. Erst ab einer Austrittsgeschwindigkeit von etwa $26 \mathrm{~m} \mathrm{~s}^{-1}$ ist es möglich, den Tropfen genügend herauszulenken. Die maximal erreichbare Einschlagsgeschwindigkeit beträgt dann etwa $40 \mathrm{~ms}^{-1}$. Auch mit zunehmender Austrittsgeschwindigkeit verläuft die Kurve deutlich unterhalb der von größeren Tropfen. Die Abnahme der erreichbaren Einschlagsgeschwindigkeit für Tropfen mit $D \gtrsim 60 \mu \mathrm{m}$ lässt sich möglicherweise dadurch erklären, dass die Bedingung $\Delta x_{\min }>D+100 \mu \mathrm{m}$ mit steigender Tropfengröße eine steigende notwendige Herauslenkung zur Folge hat, wodurch der Einfluss der Abbremsung durch die Luftreibung erhöht wird. Dieser Effekt ist jedoch gering und es wird eine maximale Einschlagsgeschwindigkeit von etwa $83 \mathrm{~m} \mathrm{~s}^{-1}$ bei $D=60 \mu \mathrm{m}$ erreicht.

Weiterhin zeigt sich, dass für alle Tropfendurchmesser $D \gtrsim 20 \mu \mathrm{m}$ Einschlagsgeschwindigkeiten von $70 \mathrm{~m} \mathrm{~s}^{-1}$ und mehr erreicht werden. Zur Bestimmung der experimentell tatsächlich $\mathrm{zu}$ erreichenden Einschlagsgeschwindigkeiten ist es abschließend notwendig, den Abstand des Substrats zu bestimmen.

14 Dies kann man verstehen, wenn man sich verdeutlicht, dass die abbremsende Kraft etwa proportional zur Querschnittsfläche $\left(\sim R^{2}\right)$, die Trägheit der Tropfen aber proportional zu ihrem Volumen ist $\left(\sim R^{3}\right)$. 


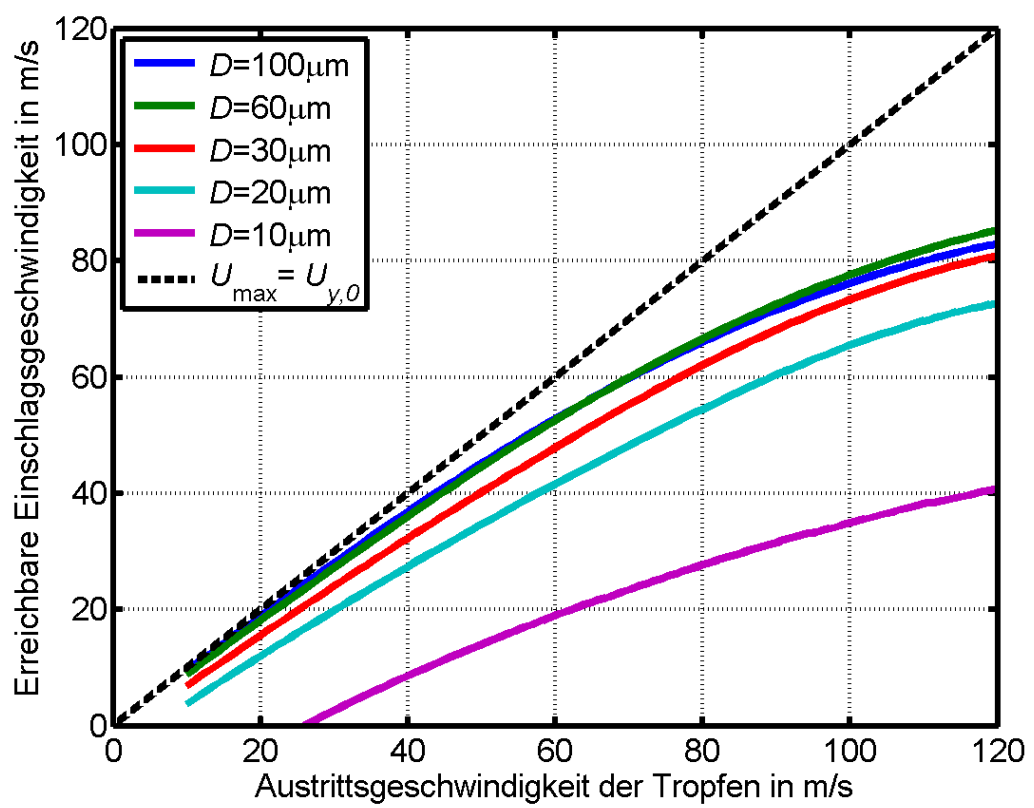

(a)

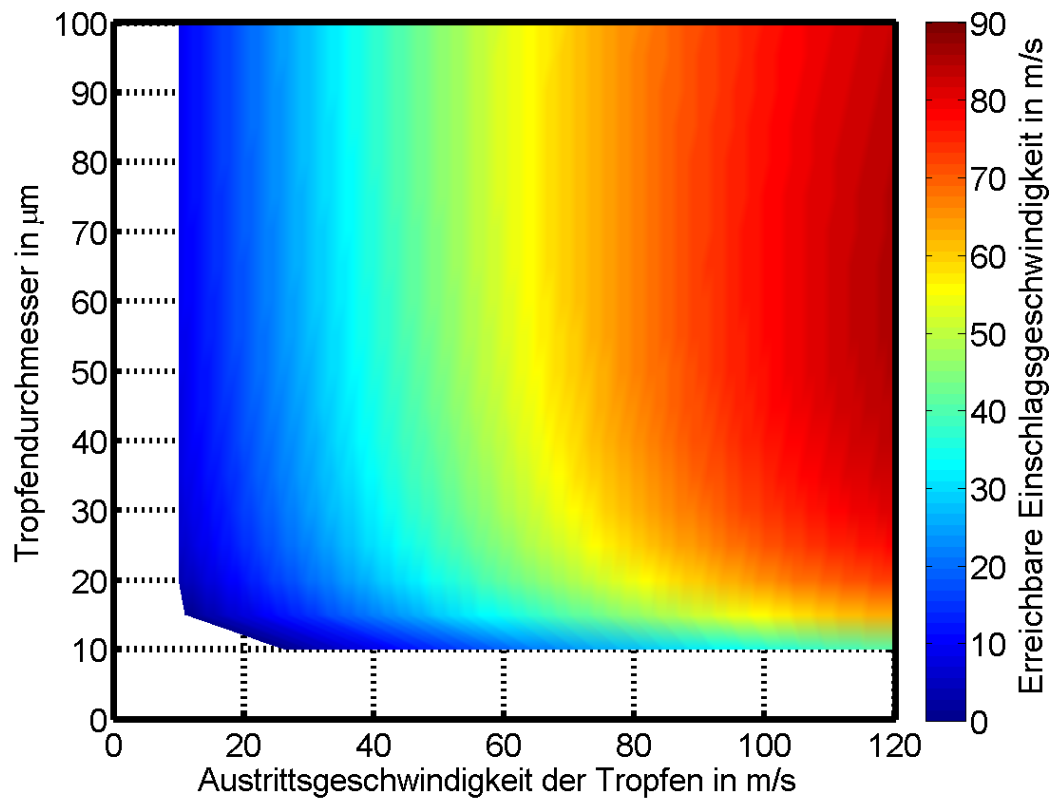

(b)

Abbildung 2.20: Maximal erreichbare Einschlagsgeschwindigkeiten für Tropfen verschiedener Durchmesser als Funktion der Austrittsgeschwindigkeit an der Düse. Die pro Tropfengröße maximal erreichbaren Einschlagsgeschwindigkeiten weisen ihr Maximum beim maximalen betrachteten Wert der Austrittsgeschwindigkeit auf. Für kleine Tropfen mit $D \lesssim 15 \mu \mathrm{m}$ lassen sich nur vergleichsweise geringe Einschlagsgeschwindigkeiten erreichen. 
Nur bei dieser Position unterhalb der Düse wird die errechnete Einschlagsgeschwindigkeit realisiert. Geringere Abstände sind durch die Vorrichtung zum Auffangen der Tropfenkette nicht möglich, größere Abstände führen aufgrund der Luftreibung zu einer verminderten Einschlagsgeschwindigkeit. Abbildung 2.21 zeigt die notwendige Position des Substrats unterhalb der Düse als Funktion der erreichbaren Einschlagsgeschwindigkeit und für verschiedene Tropfendurchmesser.

Wie in Kap. 1.4.5 gezeigt, erweist sich die Erzeugung einer monodispersen Tropfenkette sowohl im Bezug auf die Tropfengröße als auch auf die Tropfengeschwindigkeit als äußerst präzise (Abweichungen unter 1\%). Diese Genauigkeit überträgt sich auch auf den einzelnen, aufgeladenen und abgelenkten Tropfen. Sobald der Tropfen aus der Tropfenkette herausgelenkt ist, können insbesondere zwei Kräfte den exakten Zeitpunkt und die Position des Einschlags auf dem Substrat beeinflussen. Erstens können die hohen elektrischen Feldstärken, die im Experiment zum Einsatz kommen, eine weitere (unerwünschte) Beeinflussung der Tropfentrajektorie zur Folge haben. Zweitens führt der Umstand, dass sich das Substrat in einem bestimmten Abstand unterhalb der Düse befinden muss, dazu, dass auch geringe Schwankungen in der Tropfengeschwindigkeit (z.B. durch Luftbewegungen in der Nähe des Versuchsaufbaus) über die Zeit bis zum Einschlag in ihrer Stärke anwachsen. Im Experiment (siehe Kap. 2.4 erwies sich ein maximaler Abstand von etwa $25 \mathrm{~mm}$ unterhalb der Düse als erforderlich, um eine hohe Reproduzierbarkeit zu erreichen. Die durchgezogene Linie in Abb. 2.21b beschreibt die mit dem verwendeten Aufbau maximal erreichbaren Einschlagsgeschwindigkeiten. Sie weist ein globales Maximum von etwa $70 \mathrm{~m} \mathrm{~s}^{-1}$ für eine Tropfendurchmesser von $25 \mu \mathrm{m}$ auf.

Für eine weitere Optimierung des Versuchsaufbaus wäre es z.B. denkbar, den Druck der Gasatmosphäre abzusenken und damit die Reibungsverluste zu reduzieren, oder die Luft durch ein Gas geringerer Viskosität auszutauschen. Außerdem ließen sich durch zusätzliche Isolierungen der elektrischen Komponenten höhere Aufladungs- und Ablenkungsspannungen verwenden. Auch die Wahl einer Flüssigkeit mit höherem Leitwert und damit höherer Ladungsträgerdichte wäre denkbar. Bei Abwägung der Umsetzbarkeit dieser Aspekte zeigte sich ein erheblicher konstruktiver Mehraufwand, der zusätzliche Gefahrenquellen während der Versuchsdurchführung zur Folge gehabt hätte. 


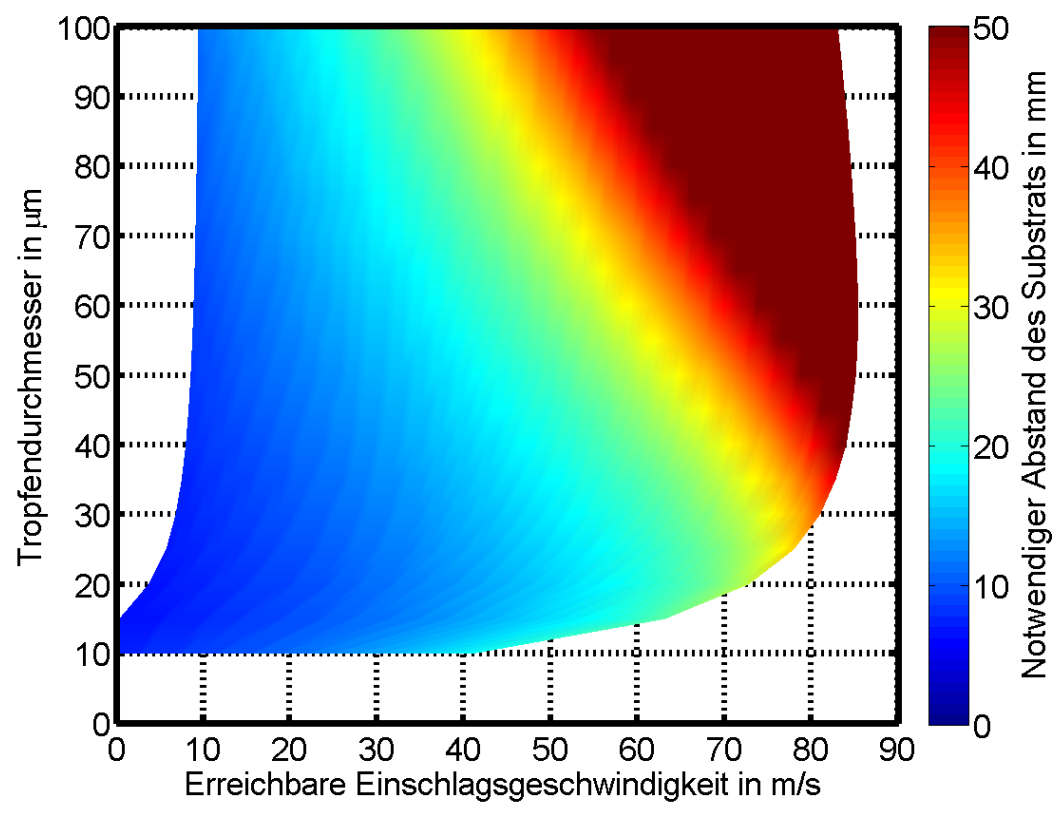

(a)

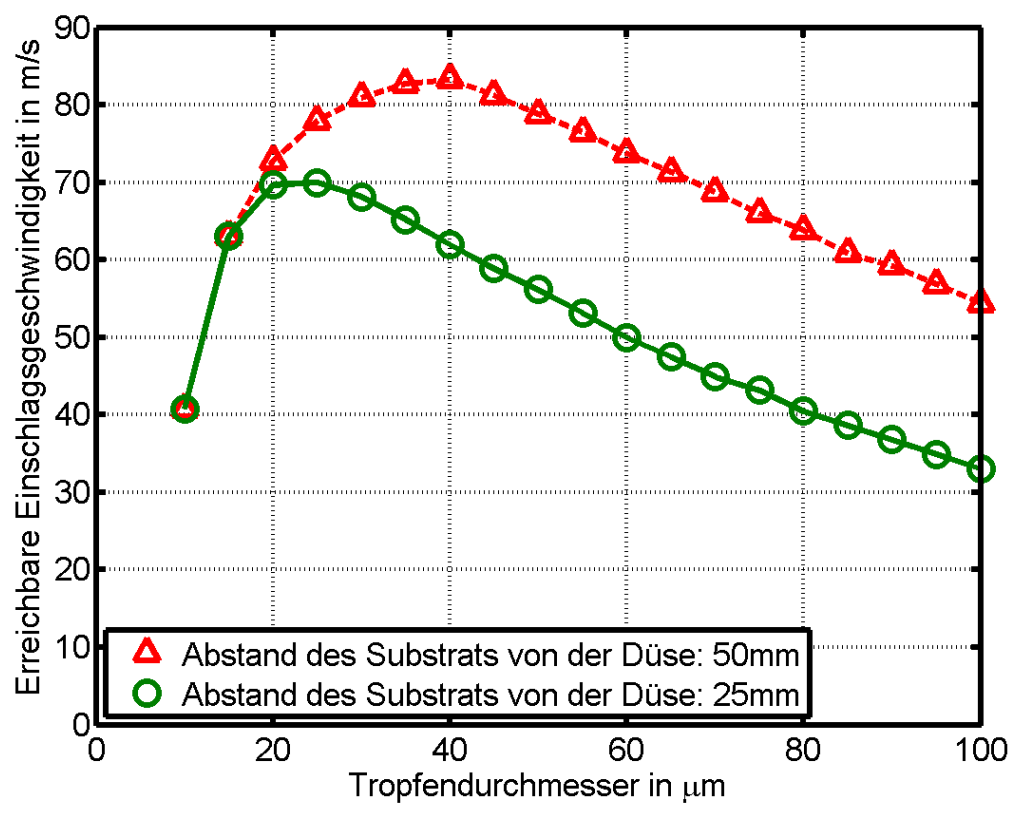

(b)

Abbildung 2.21: (a): Position unterhalb der Düse, an der sich das Substrat befinden muss, als Funktion der erreichbaren Einschlagsgeschwindigkeit und für verschiedene Tropfendurchmesser. (b): Wenn der maximale Abstand des Substrats vorgegeben ist, kommt es zu einer weiteren Einschränkung der maximal erreichbaren Einschlagsgeschwindigkeit, beispielhaft für die Abstände $50 \mathrm{~mm}$ (gestrichelte Linie) und $25 \mathrm{~mm}$ (durchgezogene Linie) gezeigt. 
Ausgehend von den in diesem Kapitel gemachten Überlegungen und gewonnenen Erkenntnissen war es möglich, den Versuchsaufbau entsprechend zu entwickeln, um präzise Experimente zum Einschlag einzelner Mikrotropfen durchzuführen. Es konnten so Tropfengeschwindigkeiten von bis zu $50 \mathrm{~m} \mathrm{~s}^{-1}$ bei einem Tropfendurchmesser von etwa $50 \mu \mathrm{m}$ erzielt werden. Die Ergebnisse zum Tropfeneinschlag werden im nachfolgenden Kap. 2.4 ausgeführt. Der Einfluss von Störungen wie z.B. Luftbewegungen nahe des Versuchsaufbaus, elektrische Aufladungseffekte und Schwankungen in der Aufladungs- und Ablenkungsspannung verhinderten, wie gerade dargelegt, noch größere Einschlagsgeschwindigkeiten. Die berechnete Geschwindigkeit von etwa $56 \mathrm{~m} \mathrm{~s}^{-1}$ bei $D=50 \mu \mathrm{m}$, entsprechend Abb. 2.21, konnte daher nicht ganz mit der für die weitere Auswertung notwendigen hohen Reproduzierbarkeit erreicht werden. 


\subsection{Auswertung: Aufprall einzelner Mikrotropfen}

Die kurzen Zeitskalen während des Einschlags schneller Mikrotropfen von etwa 1 us überfordern die erreichbaren Aufnahmegeschwindigkeiten der meisten modernen Hochgeschwindigkeitskameras [94]. Anstatt einer kontinuierlichen Aufnahme lässt sich eine stroboskopische Aufnahmetechnik verwenden, was den Vorteil einer hohen optischen Auflösung (verwendbarer Bildausschnitt auf dem Kamerachip) und einer minimierten Bewegungsunschärfe durch die kurze Belichtungszeit mit sich bringt. Diese Aufnahmetechnik erfordert jedoch eine hohe Reproduzierbarkeit beim Einschlag sowohl im Bezug auf die Tropfengröße und -geschwindigkeit als auch auf den Zeitpunkt und den Ort. Die in Kap. 2.3 beschriebene Methode erfüllt diese Voraussetzung. Durch Variation des Zeitpunkts der Aufnahme lässt sich eine Zeitreihe ähnlich zu einer kontinuierlichen Aufnahme erzeugen.

Nachfolgend werden Ergebnisse zum Aufprall einzelner Mikrotropfen auf Substrate unterschiedlicher Benetzbarkeit aufgeführt ${ }^{15}$. Es werden Tropfen mit Geschwindigkeiten von bis zu $50 \mathrm{~m} \mathrm{~s}^{-1}$ und einem Durchmesser von der Größenordnung $50 \mu \mathrm{m}$ untersucht.

\subsubsection{Ansicht von der Seite und von unten}

Zur Bestimmung von Tropfengröße und -geschwindigkeit wurden für jedes Experiment anhand der Doppelbilder der Seitenansicht, wie in Abb. 2.25a zu sehen, die Position und Kontur des Tropfens mittels eines automatisierten Skripts in MATLAB (MathWorks) ausgewertet ${ }^{16}$. Da die Zeit $\Delta t$ zwischen beiden Belichtungen bekannt ist, lässt sich aus den Positionen des Tropfens (in beiden Aufnahmen ist der Tropfen noch nicht aufgetroffen) ebenfalls seine Geschwindigkeit $V_{0}=\Delta y_{1} / \Delta t$ berechnen. Schwankungen in der Aufprallgeschwindigkeit für fest eingestellte Parameter sind beispielhaft in Abb. 2.22 (b) dargestellt. Im Anschluss wird der Median der Geschwindigkeitsverteilung verwendet, welcher aus mindestens zehn Doppelbildern gebildet wurde. Die Standardabweichung beträgt $\lesssim 1 \%$. Der Tropfendurchmesser wurde jeweils in allen Aufnahmen bestimmt, die zeitlich vor dem Aufprall liegen (pro Parameter mehr als 100 Messungen). Es wurde ebenfalls der Median gebildet, und die Standardabweichung beträgt $\lesssim 1 \%$ (siehe Abb. $2.22(\mathrm{~d})$ ).

15 Es handelt sich um Auszüge aus den Veröffentlichungen $95-97$.

16 Während der Prozess der Erzeugung einzelner Mikrotropfen, einmal eingestellt, eine nahezu perfekte Reproduzierbarkeit aufwies, konnten äußere Einflüsse (Vibrationen, Luftbewegungen, etc.) zu erheblichen Abweichungen der Tropfengröße und -geschwindigkeit führen. Diese „Ausreißer" wurden in der Auswertung nicht berücksichtigt. 
(a)

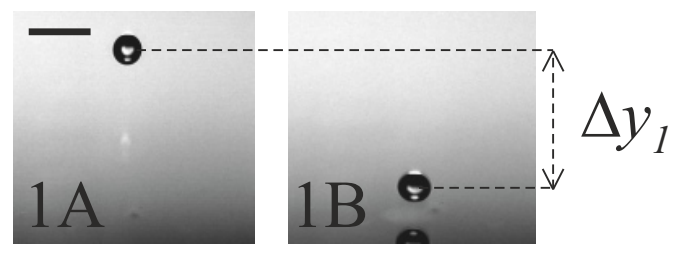

(c)

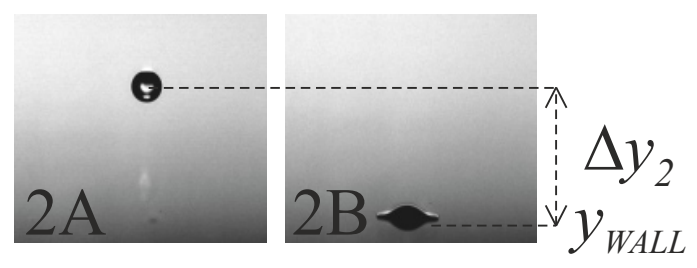

(b)

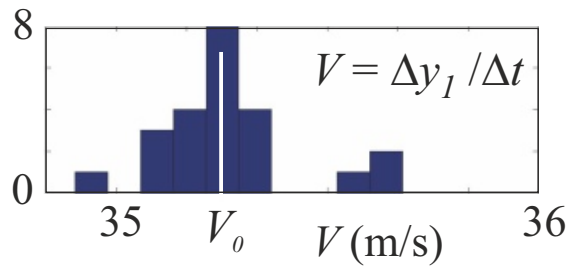

(d)

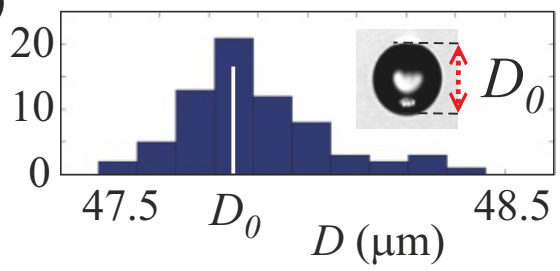

(e)
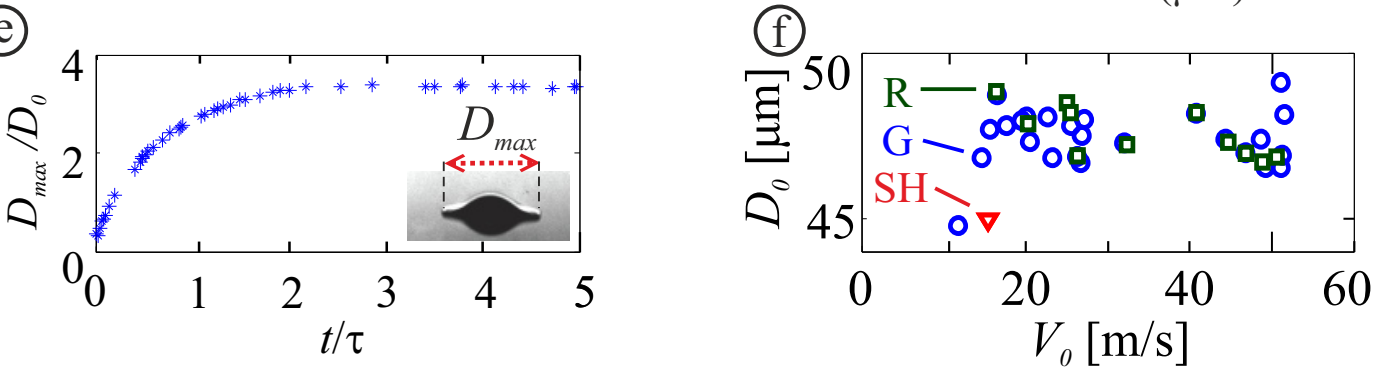

Abbildung 2.22: Auswertung der Doppelbilder A und B. (a): Aus den Aufnahmen vor dem Einschlag wird die Differenz der Tropfenpositionen $\Delta y_{1}$ zu zwei verschiedenen Zeitpunkten (1A und 1B) bestimmt. Der Maßstab (Balken oben links) entspricht $100 \mu \mathrm{m}$. (b): Mit der bekannten Zeitdifferenz wird die Verteilung der Tropfengeschwindigkeiten aus vielen Aufnahmen bei konstanten Parametern bestimmt. Der Median wird als $V_{0}$ definiert. (c): Aufnahme des Tropfeneinschlags im zweiten Doppelbild (2B). Es wird sowohl der Tropfendurchmesser $D$ als auch die Position des Substrats $y_{\text {wall }}$ bestimmt. (d): Der Median der Verteilung der Tropfengröße wird als $D_{0}$ definiert. (e): Aus dem Wandabstand und der Tropfengeschwindigkeit kann der Zeitpunkt $t$ von Bild 2B bestimmt werden. Für wiederholte Experimente zu anderen Zeitpunkten wird die Zeitentwicklung des maximalen Ausdehnungsdurchmessers $D_{\max }$ bestimmt. (f): Parameterraum $V_{0}$ und $D_{0}$ der durchgeführten Experimente auf den Substraten: hydrophil $(\mathrm{G}, \mathrm{o})$, hydrophob $(\mathrm{R}, \square)$ und superhydrophob $(\mathrm{SH}, \nabla)$.

Für Aufnahmen, in denen der Tropfen im zweiten Doppelbild bereits aufgetroffen ist, lässt sich die Position des Substrates und damit aus der Tropfengeschwindigkeit und den Tropfenpositionen der genaue Zeitpunkt des Einschlags bestimmen (siehe Abb. 2.22 (c)). Durch die Schwankungen der Tropfengeschwindigkeit $( \pm 1 \%)$ ergibt sich jedoch eine Ungenauigkeit von $\lesssim 1 \mu \mathrm{s}$. Die typische Zeitskala für den Tropfeneinschlag kann mit $\tau=D_{0} / V_{0}$ abgeschätzt werden und ist 

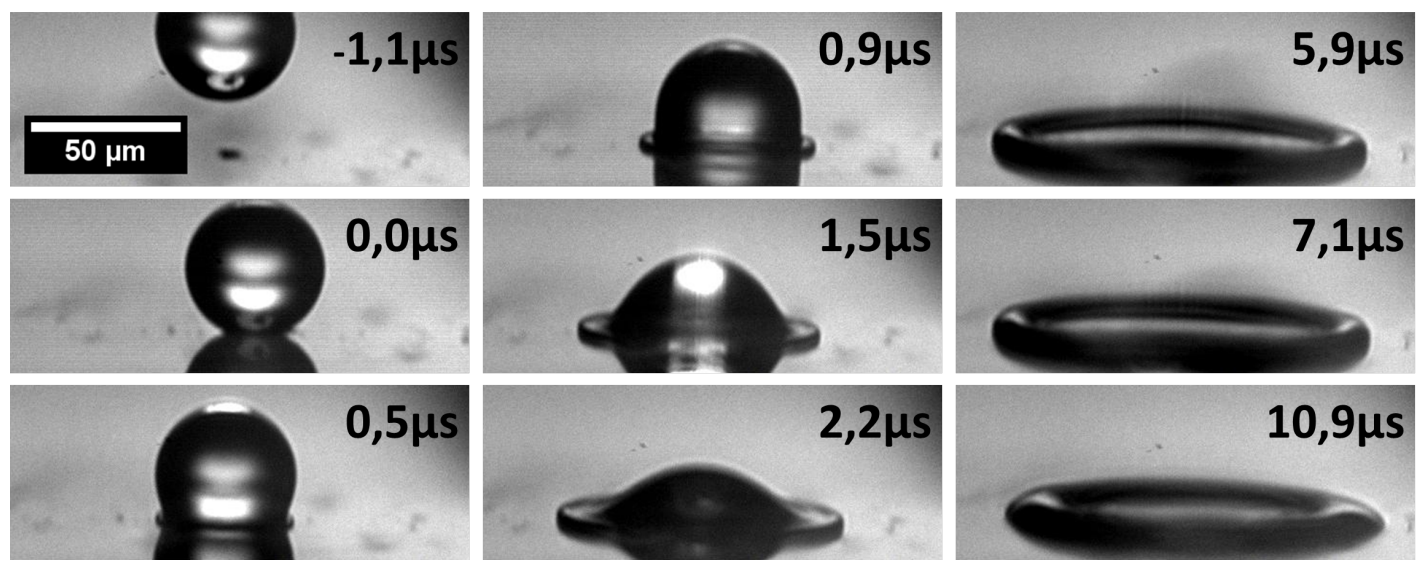

Abbildung 2.23: Unterschiedliche Zeitpunkte während des Einschlags eines Mikrotropfens mit $D_{0}=47 \mu \mathrm{m}$ und $V_{0}=15 \mathrm{~m} \mathrm{~s}^{-1}$ auf ein hydrophiles Glassubstrat.
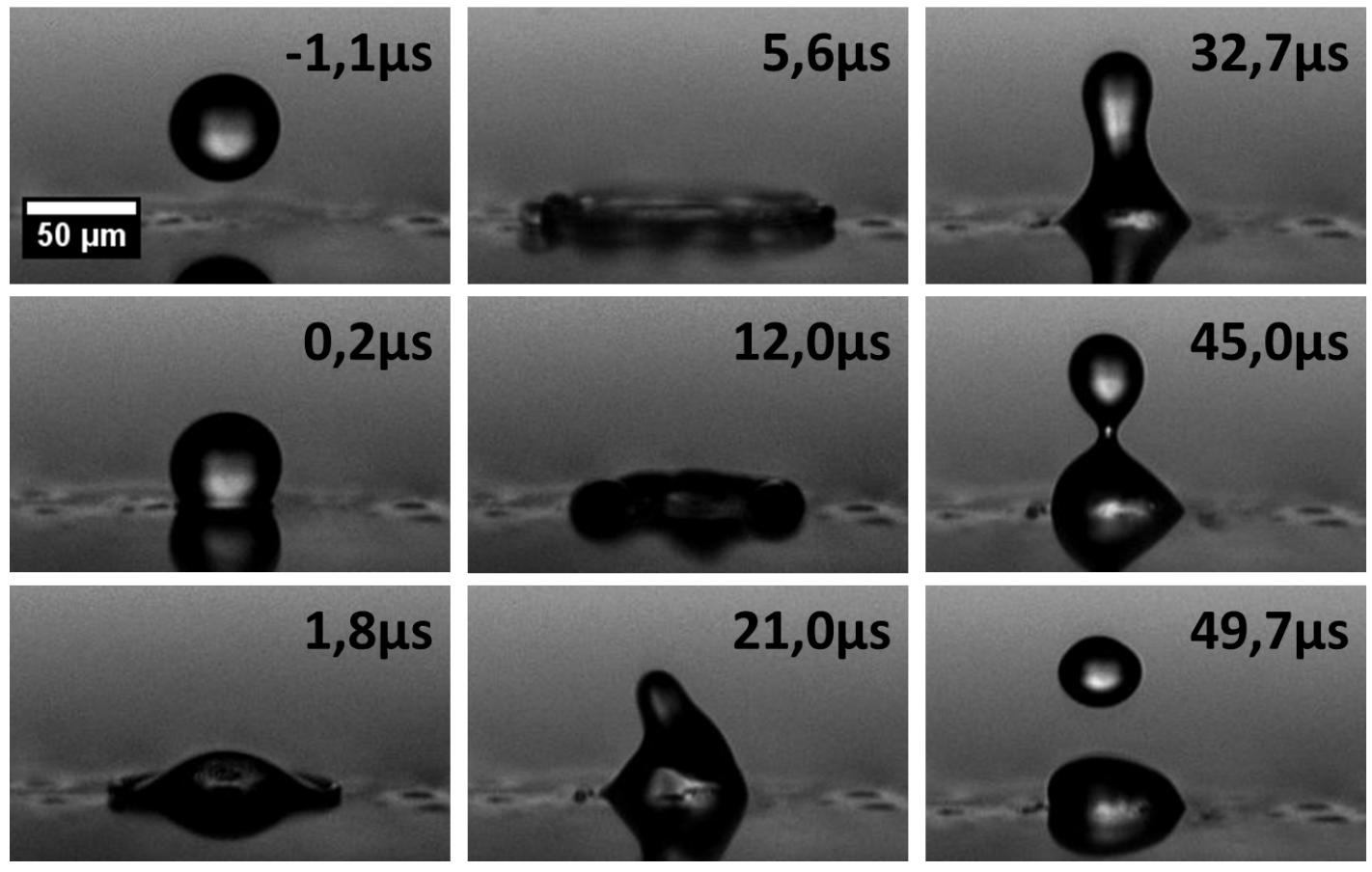

Abbildung 2.24: Unterschiedliche Zeitpunkte während des Einschlags eines Mikrotropfens mit $D_{0}=50 \mu \mathrm{m}$ und $V_{0}=15 \mathrm{~m} \mathrm{~s}^{-1}$ auf ein superhydrophobes Aluminiumsubstrat. 

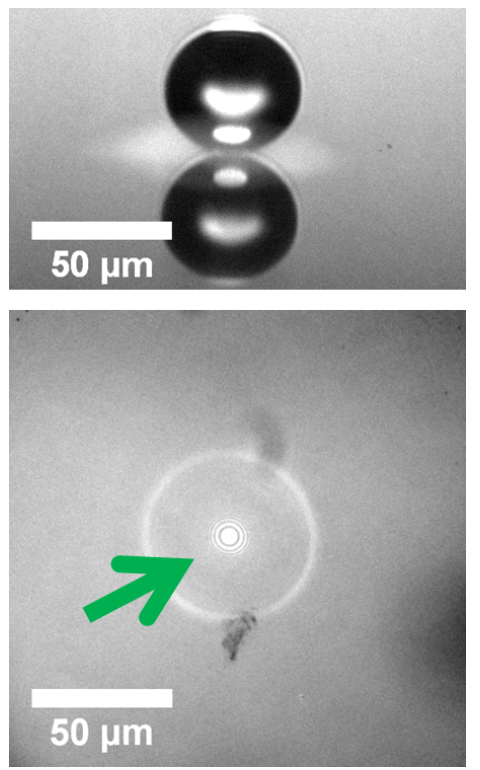

(a)
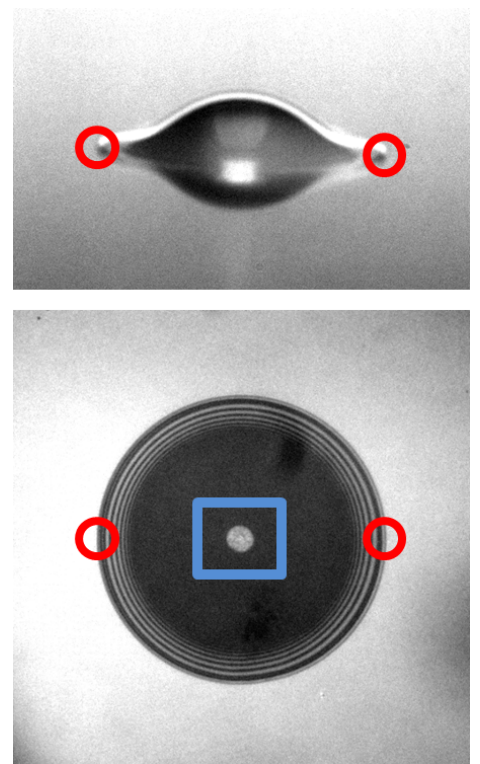

(b)
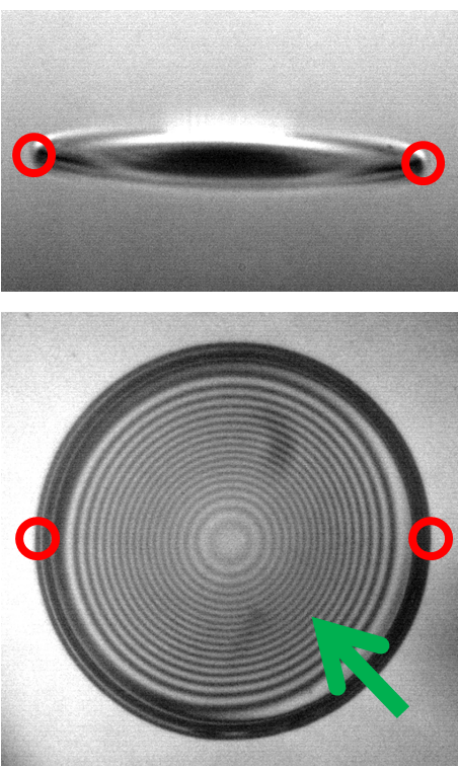

(c)

Abbildung 2.25: Aufnahmen von drei Phasen des Aufpralls eines Mikrotropfens mit $D_{0}=47 \mu \mathrm{m}$ und $V_{0}=23 \mathrm{~m} \mathrm{~s}^{-1}$. In der Ansicht von der Seite (obere Bilderreihe) und von unten (untere Bilderreihe) zeigen sich verschiedene Details: Verformung des Tropfens kurz vor und während des Einschlags (grüner Pfeil markiert resultierende Interferenzmuster), exakte Position der Kontaktlinie ist in der Ansicht von unten bestimmbar (rote Kreise) und Einschluss von Luftbläschen (blaues Rechteck).

etwa von vergleichbarer Größenordnung. Um dem entgegenzuwirken wird für jedes Doppelbild zunächst der Zeitpunkt von Bild A als $t_{\mathrm{A}}=-\tau / 2-\Delta y_{2} / V_{0}$ bestimmt. Der sich ergebende negative Zeitpunkt resultiert aus der Konvention des Einschlags bei $t=0$. Der Zeitpunkt von Bild B ergibt sich entsprechend zu $t_{\mathrm{B}}=t_{\mathrm{A}}+\Delta t$. Die Unsicherheit der Zeitbestimmung beträgt hiermit etwa $0,1 \mu \mathrm{s} \ll \tau$ und ist damit ausreichend gering für die nachfolgende Auswertung. Durch die Aufnahme zu unterschiedlichen Zeitpunkten ist es möglich, die gesamte Dynamik des Einschlags mit einer „Pseudo“-Bildrate von 100 Mfps aufzunehmen. Es handelt sich dabei jedoch nicht um eine kontinuierliche Aufzeichnung, da jedes Bild einen erneuten Tropfeneinschlag erfordert. Die Zeitspanne zwischen aufeinanderfolgenden Aufnahmen wurde so gewählt, dass die verbleibende Flüssigkeit verdunsten konnte und der erneute Einschlag wieder auf ein trockenes Substrat erfolgte.

In Abb. 2.23 und Abb. 2.24 sind beispielhaft (bereits zeitlich geordnete) Aufnahmen von der Seite dargestellt. Obwohl die Tropfengröße und -geschwindigkeit in beiden Fällen nahezu gleich ist, ist die Einschlagsdynamik aufgrund der un- 
terschiedlichen Benetzbarkeit des Substrats verschieden. Beim Einschlag auf das hydrophile Glassubstrat breitet sich der Tropfen als Flüssigkeitsfilm mit einer Wulst am Rand aus. Die Ausbreitung endet nach etwa 10 us und die Kontaktlinie bleibt beim maximalen Ausbreitungsdurchmesser fixiert. Der Einschlag auf das superhydrophobe Aluminiumsubstrat weist insbesondere am Rand sichtbare Ungleichmäßigkeiten des sich ausbreitenden Flüssigkeitsfilms auf. Der geringe Kontaktwinkel des Substrats führt nach etwa 6 us zu einer Rückbewegung der Flüssigkeit, und es kommt schließlich zum partiellen Rückprall des Tropfens.

Da die Ansicht von unten mit der Ansicht von der Seite synchronisiert ist, lassen sich die zuvor bestimmten Parameter (Tropfengröße, -position und -geschwindigkeit und Zeitpunkt der Aufnahme) übernehmen. In Abb. 2.25 sind exemplarische Aufnahmen während des Tropfeneinschlags gezeigt. Die Aufnahme der Ansicht von unten ermöglicht eine nochmals detailliertere Untersuchung des Einschlags. Durch die Verwendung von Laserlicht zur Beleuchtung kommt es zur Bildung von Interferenzmustern sowohl kurz vor als auch während des Tropfeneinschlags. Diese können genutzt werden, um die sich zeitlich ändernde Tropfenform zu ermitteln. Die Wölbung der sich ausbreitenden Tropfenfront erschwert in der Seitenansicht die Messung der exakten Position der Kontaktlinie. In der Ansicht von unten zeichnet sich die Kontaktlinie jedoch deutlich vom hellen Hintergrund ab. Abhängig von Tropfengröße und -geschwindigkeit ist es möglich, dass Luftblasen unterhalb des einschlagenden Tropfen eingeschlossen werden. Aufgrund der gewölbten Tropfenoberfläche ist dies in der Seitenansicht nur begrenzt zu beobachten.

\subsubsection{Ausbreitungsdynamik}

Der normierte Durchmesser $D / D_{0}$ des sich beim Einschlag auf dem Substrat ausbreitenden Tropfens ist in Abb. 2.26a als Funktion der normierten Zeit $t / \tau$ dargestellt. Es sind fünf Datenreihen mit unterschiedlichen Weber- und ReynoldsZahlen aufgeführt (Dreiecke und Vierecke). Der Einschlag erfolgte auf Substrate unterschiedlicher Benetzbarkeit: Hydrophil (Kontaktwinkel $\theta \approx 23^{\circ}$ ), hydrophob (Kontaktwinkel $\theta \approx 90^{\circ}$ ) und superhydrophob (Kontaktwinkel $\theta \approx 135^{\circ}$ ). Der Einfluss der Geschwindigkeitskomponente des Tropfens in $\mathrm{x}$-Richtung aufgrund der Ablenkung (siehe Kap. 2.2.1) erwies sich als vernachlässigbar. Zum einen beträgt sie zum Zeitpunkt der Herauslenkung des Tropfens aus der Tropfenkette nur etwa $1 \%$ der Geschwindigkeit in y-Richtung. Zum anderen kommt es ebenfalls in $\mathrm{x}$-Richtung zu einer starken Abbremsung aufgrund der Luftreibung (siehe Abb. 2.17a. 
Außerdem sind in Abb. 2.26a numerische Simulationen ${ }^{17}$ (durchgezogene Linien) und Messdaten eines millimetergroßen Tropfens (Sternsymbole) aus [4] enthalten.

Für Zeiten $t / \tau \lesssim 3$ zeigt sich nur ein minimaler Einfluss des Kontaktwinkels auf den Durchmesser der Ausbreitung $D / D_{0}$. Dies gilt ebenfalls für den millimetergroßen Tropfen. In allen Fällen lässt sich die Ausbreitungsdynamik sehr gut mit der numerischen Simulation beschreiben. Die Ähnlichkeit zwischen den Einschlägen der Mikrotropfen, der millimetergroßen Tropfen und den Simulationen ist ein erstes Anzeichen für eine durch dimensionslose Kennzahlen beschreibbare, skalenfreie Dynamik während des Tropfeneinschlags.

Der Tropfeneinschlag lässt sich in drei Phasen unterteilen:

I) Initial-Phase: Die Flüssigkeit im Tropfen behält annähernd die ursprüngliche Einschlagsgeschwindigkeit bei und es bildet sich noch kein ausbreitender Flüssigkeitsfilm (Lamella) auf dem Substrat. Die Zeitskala ist dabei typischerweise $t / \tau \ll 0,1$ und konnte im vorliegenden Experiment nicht aufgelöst werden.

II) Lamella-Phase: Wenn die Geschwindigkeit der sich ausbreitenden Flüssigkeit die Benetzungsgeschwindigkeit der initialen Phase überschreitet, kommt es zur Ausbildung einer dünnen Lamella $(0,1 \lesssim t / \tau \lesssim 1)$. Die Ausdehnungsgeschwindigkeit und Größe dieses Flüssigkeitsfilms lässt sich abschätzen, indem die über den Tropfen einströmende Flüssigkeitsmenge mit der in die Lamella (als Kreisscheibe mit empirischer Höhe angenommen [23|) strömenden Flüssigkeitsmenge verglichen wird

$$
D(t)=2,9 \cdot D_{0} \sqrt{t / \tau} \quad .
$$

Ein Vergleich der experimentellen Ergebnisse in doppeltlogarithmischer Auftragung, siehe Abb. 2.26b (Darstellung ist an $\mid 23$ angepasst), zeigt eine gute Übereinstimmung mit dem erwarteten Anstieg von 1/2. Durch eine Normierung mit der Reynolds-Zahl kommen alle Datenpunkte in etwa auf einer Gerade zu liegen. Auch hier zeigt sich wieder die erwähnte Skalenfreiheit.

III) Abbremsungs-Phase: Abhängig von der Weber- und Reynolds-Zahl kommt es für $t / \tau \gtrsim 1$ aufgrund der Oberflächenspannung und Viskosität der Flüssigkeit zu einer Verlangsamung der Lamella. Der finale maximale Ausbreitungsdurchmesser der Lamella $D_{\max }$ wird erreicht und hängt von der Benetzungsfähigkeit des Substrats ab.

17 Die Simulationen wurden mit der Methode Volume-Of-Fluids in Gerris 110 durch S. Wildemann durchgeführt und sind ebenfalls in der Veröffentlichung 95 enthalten. 


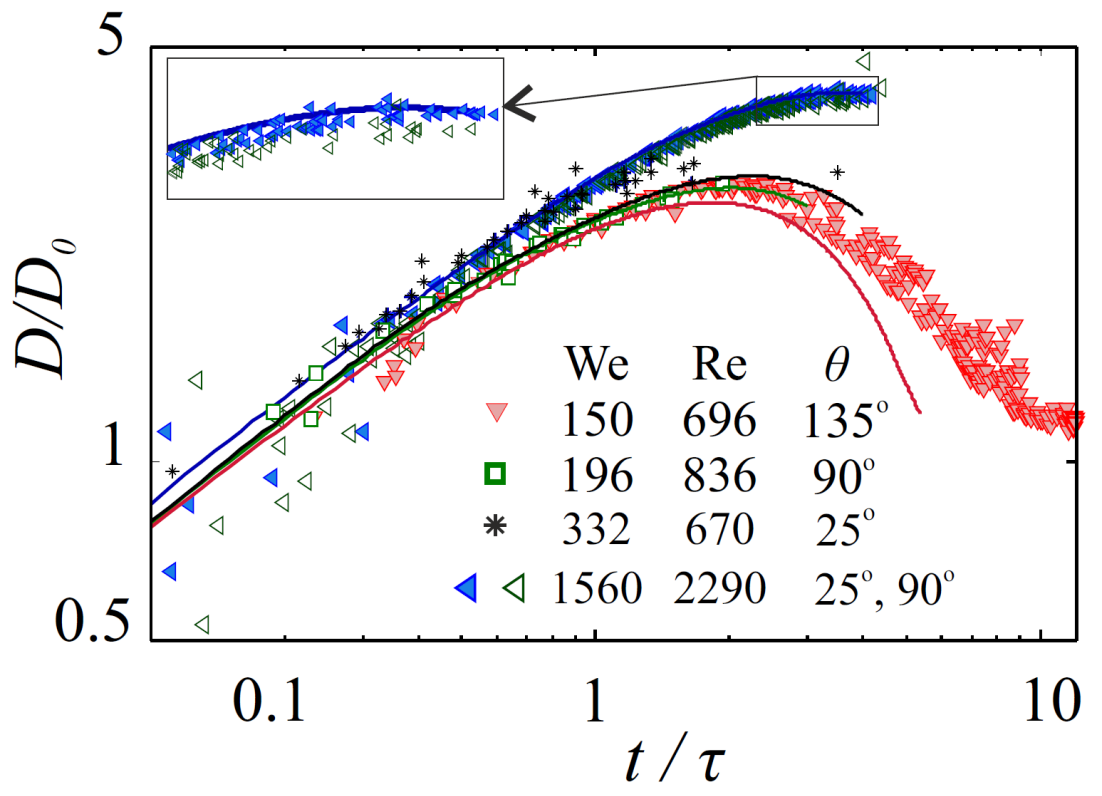

(a)

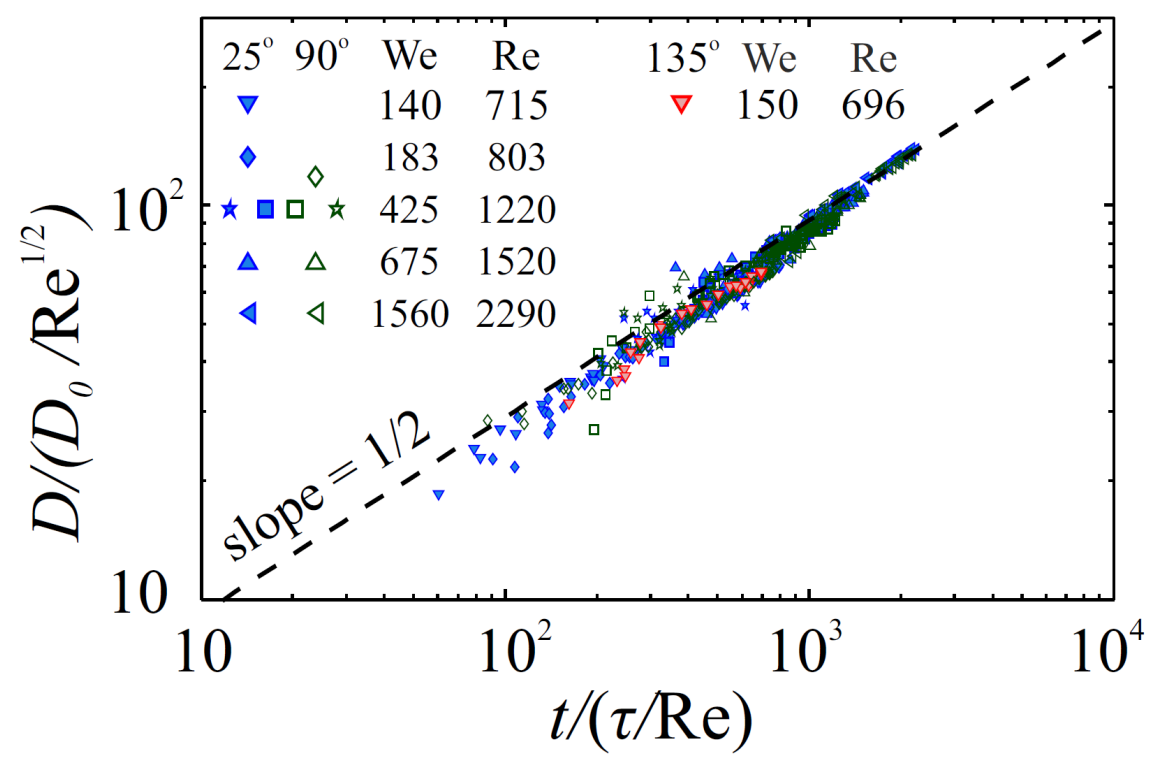

(b)

Abbildung 2.26: Auftragung des normierten Tropfendurchmessers $D / D_{0}$ während der Ausbreitung auf dem Substrat über der normierten Zeit $t / \tau$. (a): Es werden die experimentellen Ergebnisse von Miktrotropfen (Dreiecke und Vierecke) und numerischen Simulationen (durchgezogene Linien) der vorliegenden Arbeit mit einem Experiment eines millimetergroßen Tropfens (Sternsymbole) von Rioboo et al. [4] verglichen. Es zeigt sich eine gute Übereinstimmung zwischen den Experimenten der mikrometer- und millimetergroßen Tropfen und den numerischen Simulationen. (b): In der Darstellung nach [23] lässt sich für Zeiten $0,1 \lesssim t / \tau \lesssim 1$ eine Skalierung mit dem Faktor 1/2 nachvollziehen (gestrichelte Linie). 


\subsubsection{Maximaler Ausbreitungsdurchmesser}

Die Messung des maximalen Tropfendurchmessers $D_{\max }$ als Funktion der Weber-Zahl wird in Abb. 2.27 sowohl mit experimentellen Ergebnissen [53, 91 , 111-113 als auch theoretischen Modellen 6, 8, 114, 115] zum Einschlag von Mikrotropfen gegenübergestellt. Es zeigt sich eine relativ gute Übereinstimmung der experimentellen Ergebnisse in 112,113 mit denen der vorliegenden Arbeit für Weber-Zahlen der Größenordnung 100. Für größere Weber-Zahlen liegen die Messdaten der vorliegenden Arbeit über denen aus [53, 112, 113] und unter denen aus 91, 111. Die Abweichungen lassen sich unter anderem ${ }^{18}$ dadurch erklären, dass der maximale Ausbreitungsdurchmesser sowohl von der Weber- als auch von der Reynolds-Zahl abhängt, jedoch in der Abbildung nur die Abhängigkeit von der Weber-Zahl dargestellt ist. Außerdem begrenzt in den meisten Untersuchungen die zeitlich und räumlich unzureichende Auflösung bei der Aufnahme des Einschlags schneller Mikrotropfen (mit Hochgeschwindigkeitskameras) die Messgenauigkeit. Es kann angenommen werden, dass die höhere zeitliche und räumliche Auflösung der vorliegenden Untersuchung zu genaueren Messwerten führt. Der maximale Ausbreitungsdurchmesser ist das Resultat eines Gleichgewichts im Tropfen während des Aufpralls. Die impulsgetriebene radiale Strömung der Flüssigkeit wird durch die rücktreibende Oberflächenspannung und/oder durch die Dissipation aufgrund der Viskosität der Flüssigkeit ausgeglichen. In Abb. 2.27 wird entsprechend |6] der erste Fall als „capillary regime“ und der zweite Fall als „viscous regime“ gekennzeichnet. Der Übergang ist definiert als $P=W e / R e^{4 / 5}=1$ und ist mit einem Pfeil markiert. Es zeigt sich eine gute Übereinstimmung mit den vorliegenden Messdaten.

Zwei zusätzliche Effekte beeinflussen den maximalen Ausbreitungsdurchmesser: 1.) die Bildung einer Wulst am Rand der sich ausbreitenden Lamella und 2.) die Dissipation in einer dünnen Grenzschicht nahe am Substrat. Unter Berücksichtigung dieser Aspekte konnte semiempirisch gezeigt werden [8], dass sich der maximale Ausbreitungsdurchmesser für $W e \gtrsim 10$ beschreiben lässt als

$$
D_{\max } / D_{0}=0,87 \cdot R e^{1 / 5}-0,4 \cdot R e^{2 / 5} W e^{-1 / 2} \quad .
$$

Dieses Modell zeigt eine nochmals genauere Beschreibung der vorliegenden experimentellen Ergebnisse. Aufgrund der Tatsache, dass es für (langsame) millimetergroße Tropfen validiert wurde, kann geschlussfolgert werden, dass sich der maximale Ausbreitungsdurchmesser auch über diesen erweiterten Parameterbereich (für schnelle Mikrotropfen) allein durch Weber- und Reynolds-Zahl beschreiben lässt.

\footnotetext{
18 Weitere Erklärungen sind in Anh. A in 95 zu finden.
} 


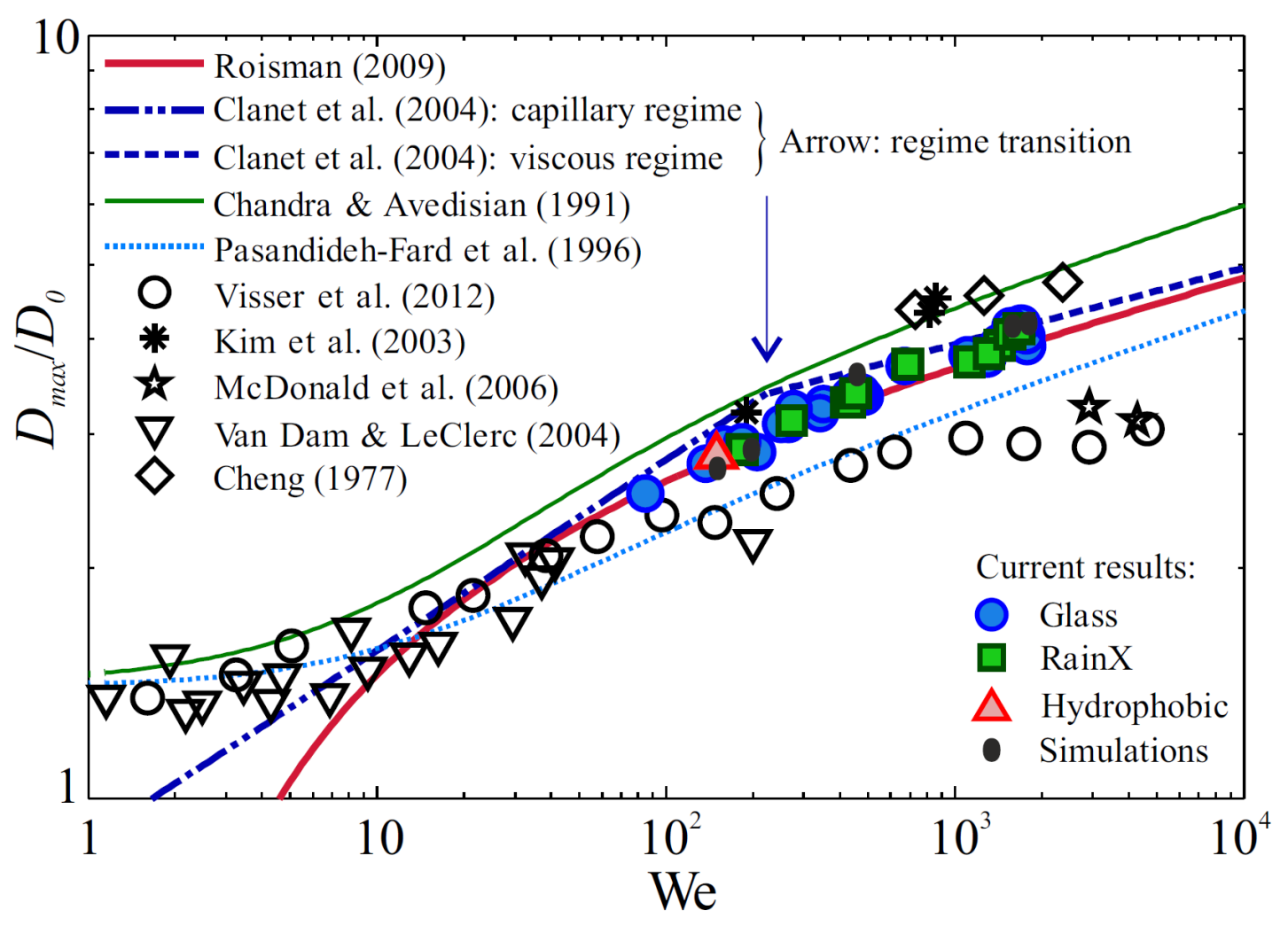

Abbildung 2.27: Auftragung des normierten maximalen Tropfendurchmessers $D_{\max } / D_{0}$ während der Ausbreitung auf dem Substrat gegen die Weber-Zahl. Es werden die experimentellen Ergebnisse der vorliegend Arbeit (farbige Symbole) und numerischen Simulationen von S. Wildemann (schwarze Punkte) zusammen mit anderen Experimenten und verschiedenen Modellen verglichen.

\subsubsection{Tropfenprofil}

Das Tropfenprofil kann in den frühen Phasen des Tropfeneinschlags näherungsweise aus den Aufnahmen der Seitenansicht abgeleitet werden. Jedoch ist eine genaue Bestimmung der Dicke und Wölbung des sich ausbreitenden Flüssigkeitsfilms und des Wulstes nur bedingt möglich. Die Tatsache, dass als Lichtquelle für die Aufnahme von unten monochromatisches Laserlicht mit einer Wellenlänge von $\lambda=532 \mathrm{~nm}$ verwendet wird, ermöglicht es, aus dem sich bildenden Interferenzmuster (siehe Abb. 2.25 Rückschlüsse auf die Dicke des Flüssigkeitsfilms zu ziehen. Es gibt zwei gekennzeichnete Übergänge zwischen Materialien mit unterschiedlichem Brechungsindex 19 ; 1) Luft-Glas an der Unterseite des Substrats und 2) Wasser-Luft an der Oberseite des Tropfens. Durch konstruktive und de-

19 Da der Unterschied im Brechungsindex beim Übergang von Glas zu Wasser (an der Unterseite des Tropfens) vergleichsweise gering ist, wird dieser Beitrag der Reflexion vernachlässigt. 


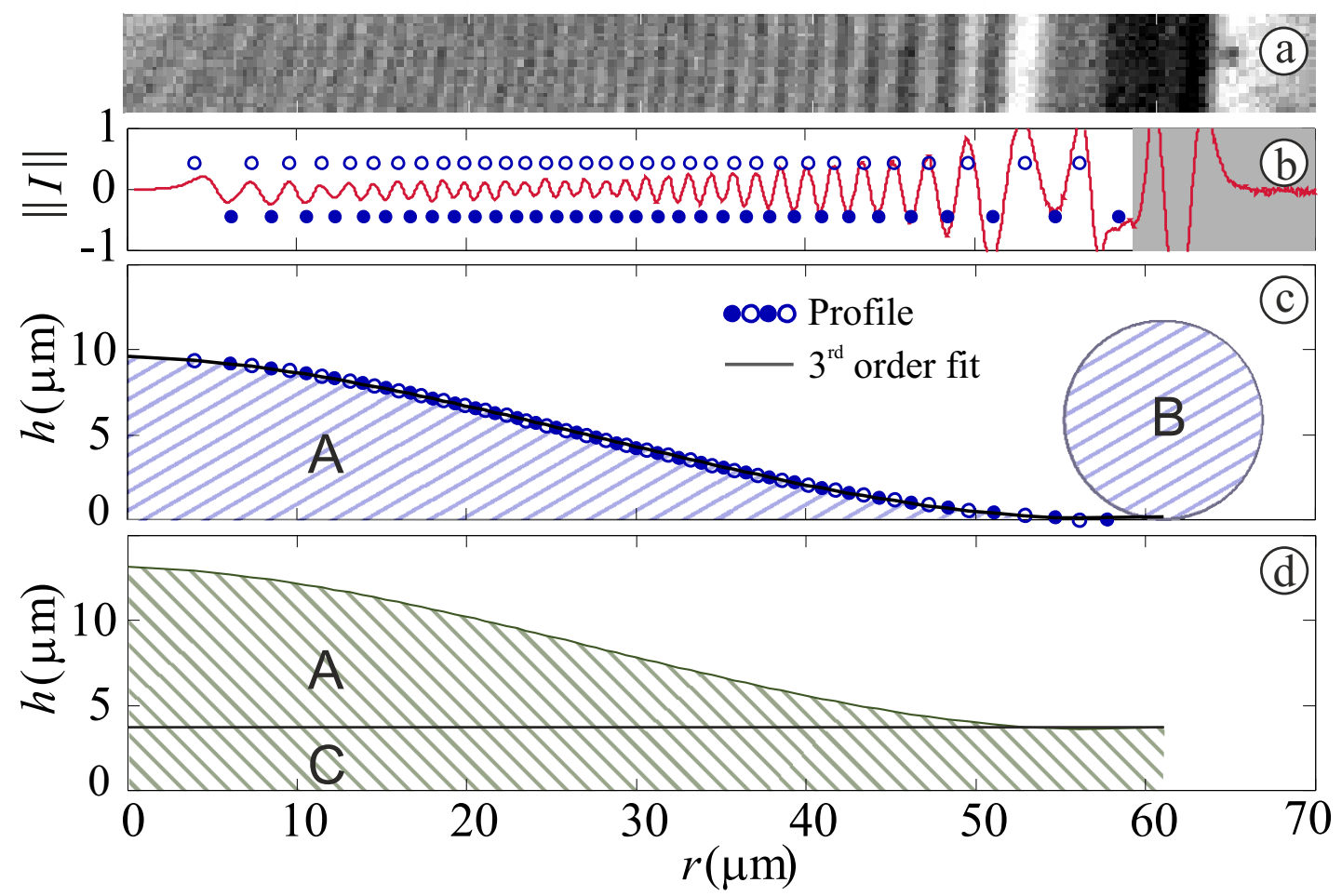

Abbildung 2.28: Bildanalyse der Interferenzmuster in der Ansicht von unten. (a): In der Vergrößerung sind die Interferenzstreifen deutlich erkennbar. (b) Nach radialer Mittelung zeigt sich ein alternierender Intensitätsverlauf (Minima und Maxima sind durch Punkte und Kreise gekennzeichnet). Der graue Bereich für $r \gtrsim 60 \mu \mathrm{m}$ wird nicht berücksichtigt. (c): Unter Annahme einer monoton abnehmenden Filmdicke ist das berechnete Höhenprofil gezeigt, beschrieben durch einen PolynomialFit dritter Ordnung (durchgezogene Linie). Die axialsymmetrische Integration der Fläche A ergibt ein Volumen, welches kleiner als das Gesamtvolumen des Tropfen ist. Das verbleibende Volumen ist in Form einer Wulst (Fläche B) dargestellt. (d): Im Grenzfall, dass es keine Wulst gibt, muss das verbleibende Volumen als zusätzliche Filmdicke unterhalb des Höhenprofils vorliegen (Fläche C).

struktive Interferenz kommt es für Höhenunterschiede im Flüssigkeitsfilm von $h=\lambda / 4 \sim 133 \mathrm{~nm}$ zu den sichtbaren dunklen und hellen Ringen, wie in Abb. 2.25 zu sehen. Diese sind in Abb. 2.28 (a) vergrößert dargestellt.

Nach radialer Mittelung ergibt sich das in Abb. 2.28 (b) gezeigte Intensitätsprofil. Die Minima und Maxima werden mittels eines Skripts in MATLAB (MathWorks) bestimmt und jeder Hell-Dunkel-Übergang wird einer Reduzierung der Filmdicke von einer viertel Wellenlänge zugeordnet. Das resultierende Profil ist in Abb. 2.28 (c) gegeben, wobei für den äußersten Interferenzring eine Höhe von $0 \mu \mathrm{m}$ angenommen wurde. Die Annahme, dass die Filmdicke bis zur 
Wulst monoton abnimmt lässt sich mit Hilfe der Aufnahme von der Seite begründen. Auf diese Weise ${ }^{20}$ lässt sich jedoch nur die Kontur, nicht aber die absolute Filmdicke bestimmen.

Durch axialsymmetrische Integration des bisherigen Tropfenprofils lässt sich dessen Volumen bestimmen und mit dem bekannten Tropfenvolumen vergleichen. Das Differenzvolumen muss in Form der Wulst und/oder als Filmdicke unterhalb des Tropfenprofils vorliegen, jeweils als $\mathrm{B}$ und $\mathrm{C}$ in Abb2.28 gekennzeichnet. Das tatsächliche absolute Tropfenprofil liegt zwischen beiden dargestellten Grenzfällen.

Mit Hilfe der Seitenansicht lässt sich die Größe der Wulst entsprechend des Kreises in Abb. 2.29 (a) abschätzen. Anschließend kann die Filmdicke unter dem Tropfenprofil berechnet werden. Eine dreidimensionale Darstellung (siehe Abb. 2.29 (b)) des auf diese Weise bestimmten absoluten Tropfenprofils zeigt eine sehr gute Übereinstimmung mit der Aufnahme von der Seite ${ }^{21}$.

Eine ebenso gute Übereinstimmung ist mit der numerischen Simulation in Abb. 2.29 (c) zu erkennen. Da die Berechnung auf dimensionslosen Kennzahlen beruht, zeigt sich ein weiteres Mal die Skalenfreiheit bei der Beschreibung des Tropfeneinschlags.

Die beschriebene Methode zur Bestimmung des absoluten Tropfenprofils lässt sich besonders gut für Phasen des Einschlags durchführen, in welchen der Tropfen eine relativ flache Form und nicht zu starke Krümmungen aufweist (vergleichbar mit Abb. 2.25c). In der anfänglichen Phase des Einschlags (etwa zwischen den Zeitpunkten in Abb. 2.25a und Abb. 2.25b ist die Krümmung zu hoch bzw. die Oberfläche nicht durchgehend konvex, sodass die Interferenzstreifen nicht optisch aufgelöst und ausgewertet werden können. Für diese Fälle ist eine relativ gute Bestimmung der Tropfenform aus den Aufnahmen von der Seite möglich.

Unter Verwendung beider Methoden lässt sich über weite Bereiche des Einschlagprozesses das tatsächliche dreidimensionale Tropfenprofil (unter Annahme von Rotationssymmetrie) bestimmen. Die hohe räumliche und zeitliche Auflösung, wie sie auch schon in den vorangegangenen Kap. 2.4 .2 und Kap. 2.4.3 vorlag, führt auch in diesem Fall zu einer bis dato unerreichten Messgenauigkeit.

${ }^{20}$ Eine absolute Bestimmung der Höhe ist z.B. mit einer nicht-monochromatischen Lichtquelle möglich [31.

21 Die Beleuchtung wurde entsprechend der im Experiment gemachten Aufnahme gewählt. Zusätzlich wurde die sichtbare Reflexion in schwarz hinzugefügt. 

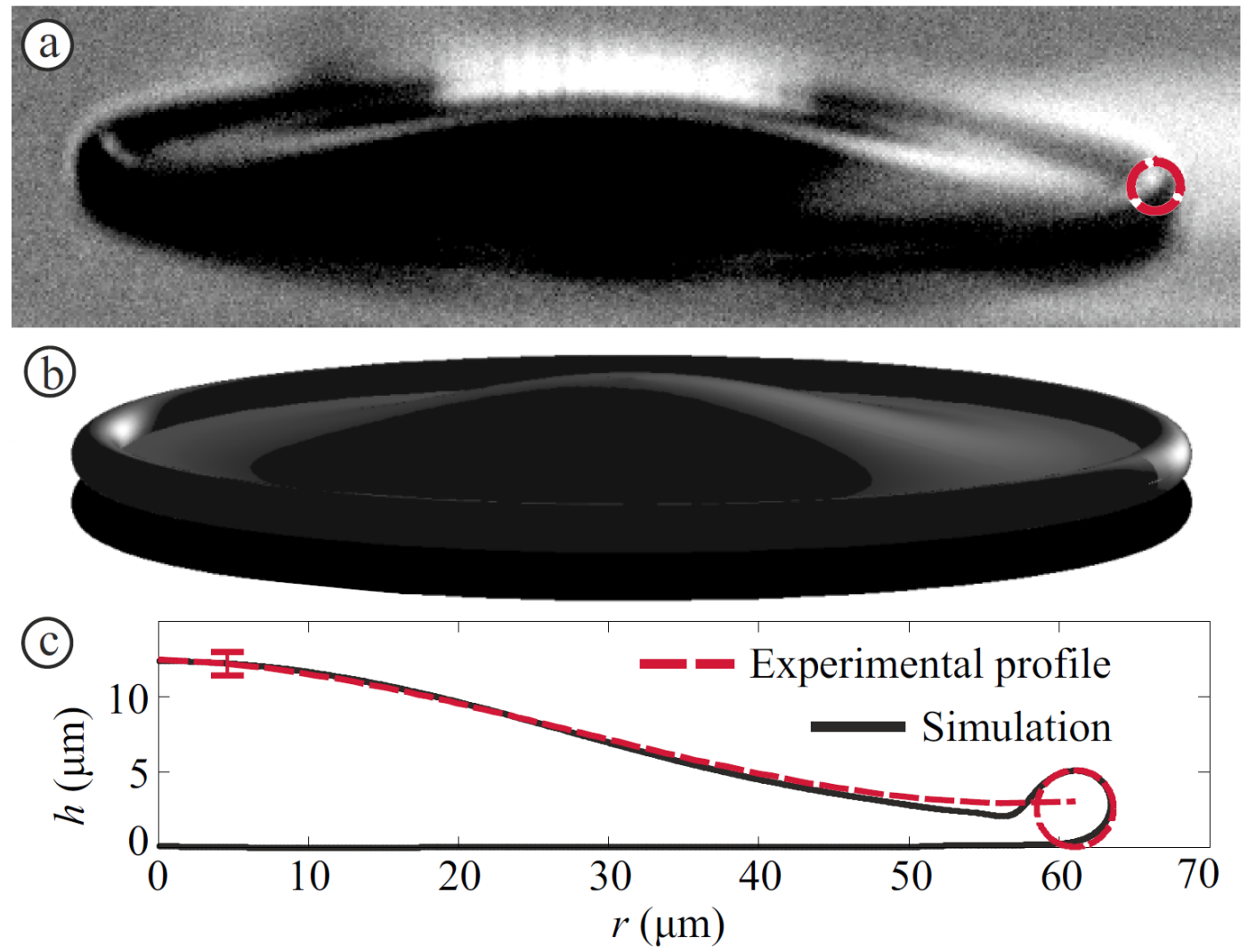

Abbildung 2.29: Vergleich zwischen der Seitenansicht (a), dem berechneten Tropfenprofil (b) und der numerischen Simulation (c). (a): Aus der Seitenansicht lässt sich die Größe der Wulst abschätzen (roter Kreis). (b): Die Rekonstruktion des aus den Interferenzstreifen berechneten Tropfenprofils zeigt eine sehr gute Übereinstimmung mit der Seitenansicht. (c): Die Abweichung zwischen dem mittleren (Fehlerbalken entspricht experimenteller Unsicherheit) rekonstruierten Tropfenprofil und der numerischen Simulation sind ebenfalls sehr gering. 


\subsection{Zusammenfassung}

\section{Erzeugung einzelner Mikrotropfen}

Die reproduzierbare Erzeugung einzelner Mikrotropfen variabler Größe und Geschwindigkeit ist notwendig zur Untersuchung des Aufprallprozesses. Es konnte gezeigt werden, wie sich ein einzelner Mikrotropfen elektrisch aufladen und ablenken lässt. Dazu wurde entsprechend Kap. 1 ein Flüssigkeitsstrahl erzeugt, der unter gezielter externer Anregung in eine Kette monodisperser Tropfen zerfällt. In der Nähe des Strahlzerfalls in Tropfen wurde durch einen zeitlich begrenzten Hochspannungspuls eine Ladungsträgerverschiebung in der Spitze des Strahls hervorgerufen, sodass eine dem Spannungspuls entgegengesetzte Ladung auf dem sich ablösenden Tropfen verbleibt und die nachfolgend sich ablösenden Tropfen wieder neutral sind. Anschließend ist es möglich, den einzelnen, geladenen Tropfen aus der Tropfenkette herauszulenken. Nach Entfernung der Tropfenkette durch eine geeignete Auffangvorrichtung lässt sich mit dieser Methode der kontrollierte Aufprall eines Mikrotropfens untersuchen. Für die Optimierung der Reproduzierbarkeit des Einschlags und eine möglichst hohe Einschlagsgeschwindigkeit wurden die Aspekte der Aufladung, der Ablenkung und der Abbremsung (aufgrund der Viskosität des umgebenden Gases, hier Luft bei Normaldruck) getrennt untersucht und schließlich in einem Modell vereint. Der Prozess der Tropfenaufladung lässt sich mit dem eines Kondensators vergleichen. Die Aufladungselektrode bildet mit dem Flüssigkeitsstrahl im Zentrum einen Zylinderkondensator, der über den Widerstand des Flüssigkeitsstrahls zwischen Aufladungselektrode und Düse aufgeladen wird. Aufgrund der Annahmen und Vereinfachungen in den Modellen 82, 105, 106 zur a priori Bestimmung der Zeitkonstante der Aufladung $\tau$ und der maximal übertragbaren Ladungsmenge $Q_{\max }$ kommt es zu Abweichungen etwa um den Faktor zwei bis fünf von den experimentell bestimmten Werten. Die Zeitkonstante der Aufladung ist sowohl proportional zum Leitwert der Flüssigkeit als auch zur Länge des Flüssigkeitsstrahls zwischen Aufladungselektrode und Düse. Beide Größen lassen sich nur vor der Versuchsdurchführung bestimmen. Durch die Flüchtigkeit des in geringen Mengen beigefügten Ammoniaks (um ein Mindestmaß an verfügbaren Ladungsträger zu gewährleisten) ist die Konzentration und somit die Leitfähigkeit der Flüssigkeit sowohl zeitlich als auch räumlich nicht konstant. Des Weiteren ist die genaue Bestimmung der Länge des Flüssigkeitsstrahls zwischen Aufladungselektrode und Düse durch die Versuchsanordnung optisch nicht zugänglich. Dennoch ist der Prozess der Aufladung mit a posteriori bestimmtem $\tau$ und $Q_{\max }$ sehr gut beschreibbar, da sowohl der exponentielle Zusammenhang zwischen der Ladungs- 
menge $Q$ und der Zeitkonstante $\tau$ als auch der lineare Zusammenhang zwischen der Aufladungsspannung $U_{\text {auf }}$ und der maximal übertragbaren Ladungsmenge $Q_{\max }$ nachvollzogen werden konnten. In allen Fällen wurde die Grenze für einen Rayleigh-stabilen Tropfen [101 nicht überschritten, und eine sphärische Tropfenform war gewährleistet.

Auf einen Tropfen innerhalb der Tropfenkette und auf einen Einzeltropfen in der Nähe der Tropfenkette wirkt aufgrund der Viskosität des umgebenden Gases eine Reibungskraft. Es wurde gezeigt, dass die häufig verwendeten empirischen Gleichungen (z.B. 45|) eine nur ungenügende Beschreibung des Strömungswiderstandskoeffizienten $C_{\mathrm{D}}$ und damit der Abbremsung eines Tropfens ermöglichen. Erst die Berücksichtigung des sich durch den Impulsübertrag der Flüssigkeit auf das umgebende Gas bildenden Geschwindigkeitsfeldes [109 lässt eine ausreichend genaue Berechnung der Tropfengeschwindigkeit als Funktion des Abstands von der Düse zu. Ein Tropfen innerhalb der Tropfenkette ist nur einer geringen Reibungskraft ausgesetzt, da er sich im mitbewegten Luftstrom der vorangegangenen Tropfen bewegt. Anders verhält es sich für einen herausgelenkten Tropfen, der eine deutlich stärkere Abbremsung erfährt, insbesondere je kleiner sein Durchmesser ist. Die Abbremsung von Tropfen innerhalb der Tropfenkette ließ sich für Austrittsgeschwindigkeiten aus der Düse $U_{0}$ von etwa $30 \mathrm{~m} \mathrm{~s}^{-1}, 70 \mathrm{~m} \mathrm{~s}^{-1}$ und $100 \mathrm{~m} \mathrm{~s}^{-1}$ und für Tropfengrößen $D$ zwischen $30 \mu \mathrm{m}$ und $40 \mu \mathrm{m}$ über einen Abstand von bis zu $50 \mathrm{~mm}$ unterhalb der Düse sehr genau beschreiben (Abweichungen $<1 \%$ ). Es war ebenfalls möglich, die Abbremsung eines Einzeltropfens mit $U_{0}=25 \mathrm{~m} \mathrm{~s}^{-1}$ und $D=37 \mu \mathrm{m}$ in Abhängigkeit vom Abstand $(\lesssim 800 \mu \mathrm{m})$ zur Tropfenkette mit dem Experiment zu vergleichen. Hierbei zeigte sich, dass die Geschwindigkeitskomponente in $\mathrm{x}$-Richtung (aufgrund der Ablenkung durch das elektrische Feld) nicht zu vernachlässigen ist. Erst durch Berücksichtigung dieser Tatsache konnte ein vereinigtes Modell formuliert werden, das die Prozesse der Erzeugung einer monodispersen Tropfenkette, der Aufladung eines einzelnen Tropfens, der Ablenkung des geladenen Tropfens und der Reibungskraft in dem umgebenden Gas beinhaltet. Der Vergleich mit der experimentell bestimmten Tropfentrajektorie zeigt eine nahezu perfekte Beschreibung.

Im Weiteren wurde, ausgehend vom dem vereinigten Modell, untersucht, wovon die Geschwindigkeit beim Einschlag abhängt und inwieweit sie sich maximieren lässt. Für die Durchführung von Experimenten zum Einschlag von Einzeltropfen ist es notwendig, den elektrisch geladenen Tropfen um einen Abstand $x_{\text {min }}$ aus der Tropfenkette herauszulenken, um die Tropfenkette in ein Auffanggefäß umzuleiten. Aufgrund der erforderlichen räumlichen Separierung kommt es zu einer Abbremsung des Einzeltropfens, bevor er auf dem Substrat auftrifft. Das vereinigte Modell wurde benutzt, um für verschiedene Austrittsgeschwindigkeiten 
der Tropfen aus der Düse, $10 \mathrm{~m} \mathrm{~s}^{-1} \leq U_{0} \leq 120 \mathrm{~m} \mathrm{~s}^{-1}$, und verschiedene Tropfengrößen, $10 \mu \mathrm{m} \leq D \leq 100 \mu \mathrm{m}$, die resultierende maximal erreichbare Einschlagsgeschwindigkeit $U_{\max }$ und die notwendige Position des Substrats zu berechnen. Unter Verwendung der experimentell maximal möglichen Aufladungsspannung $U_{\text {aufl }}=1 \mathrm{kV}$ und der maximalen Ablenkungsfeldstärke $E_{x, \text { abl }}=1 \mathrm{MV} \mathrm{m}^{-1}$ zeigte sich in allen Fällen, dass $U_{\max }<U_{0}$ ist. So beträgt für einen Tropfen von $100 \mu \mathrm{m}$ Durchmesser die maximal erreichbare Einschlaggeschwindigkeit $U_{\max } \approx 80 \mathrm{~m} \mathrm{~s}^{-1}$ und für einen Tropfen von $10 \mu \mathrm{m}$ Durchmesser $U_{\max } \approx 40 \mathrm{~m} \mathrm{~s}^{-1}$, bei jeweils identischer Austrittsgeschwindigkeit von $U_{0}=120 \mathrm{~m} \mathrm{~s}^{-1}$. Je kleiner der Tropfen ist, desto stärker ist die Abbremsung aufgrund der Luftreibung. Es lassen sich jedoch für alle Tropfen $D \gtrsim 20 \mu \mathrm{m}$ theoretisch relativ hohe Einschlagsgeschwindigkeiten von $70 \mathrm{~m} \mathrm{~s}^{-1}$ und mehr erreichen. Für eine Umsetzung im Experiment muss jedoch beachtet werden, dass die Position, an der $U_{\max }$ erreicht wird, in starkem Maße von der Austrittsgeschwindigkeit und Tropfengröße abhängig ist. Unter der Maßgabe einer hohen räumlichen und zeitlichen Reproduzierbarkeit des Einschlags erwies sich ein maximaler Abstand des Substrats unterhalb der Düse von $25 \mathrm{~mm}$ als geeignet. Für einen Tropfendurchmesser von $50 \mu \mathrm{m}$ ergibt sich eine theoretische maximale Einschlagsgeschwindigkeit von $56 \mathrm{~m} \mathrm{~s}^{-1}$. Im Experiment ließs sich eine Maximalgeschwindigkeit von bis $\mathrm{zu} 50 \mathrm{~m} \mathrm{~s}^{-1}$ erreichen.

Zusammenfassend lässt sich festhalten, dass die Untersuchung und Zusammensetzung der Teilaspekte der Aufladung, Ablenkung und Abbremsung eines Einzeltropfens eine sehr gute Beschreibung der Trajektorie ermöglicht. Weiterhin ließ sich mit den gewonnen Erkenntnissen der Versuchsaufbau bis nahe an die technische Begrenzung entwickeln, die aufgrund der Verwendung von zeitlich kurzen und präzisen Hochspannungspulsen vorliegt. Ausgehend von der zu untersuchenden Tropfengröße und -geschwindigkeit lassen sich die notwendigen experimentellen Parameter bestimmen. Die Kombination der hohen Reproduzierbarkeit dieser Methode mit der Erzeugung einer monodispersen Tropfenkette ermöglicht kontrollierte Experimente zum Einschlag einzelner Mikrotropfen über einen breiten Parameterraum. 


\section{Einschlag einzelner Mikrotropfen}

Es wurden in jüngerer Vergangenheit bereits große Fortschritte im Verständnis zum Einschlag von millimetergroßen Tropfen bei niedrigen Geschwindigkeiten $\lesssim 10 \mathrm{~m} \mathrm{~s}^{-1}$ gemacht, z.B. Ausbreitungsdynamik während des Einschlags [4 9,23$]$, „splashing“ 12, 14, 19, Einschluss eines Luftbläschens unter dem Tropfen 27-31 oder Messung der Grenzschichtströmung 178, 92, 93. Jedoch existieren nur vergleichsweise wenige Untersuchungen (z.B. |70,91|) zu mikrometergroßen Tropfen bei hohen Geschwindigkeiten $\gtrsim 10 \mathrm{~m} \mathrm{~s}^{-1}$. Dies steht im Kontrast zu der Tatsache, dass letztere ein wichtiger Bestandteil vieler moderner Anwendungen sind. Daher ist ein ebenso gutes Verständnis des Einschlagprozesses notwendig.

Mit der in Kap. 2.3 beschriebenen Methode zur Erzeugung von Einzeltropfen ist es möglich, reproduzierbar den Einschlag schneller Mikrotropfen aufzunehmen. Aufgrund der typischen Zeitskalen von etwa $1 \mu \mathrm{s}$ ist die Verwendung selbst modernster Hochgeschwindigkeitskameras nur begrenzt möglich 94, 113. Einen sowohl räumlich als auch zeitlich reproduzierbaren Einschlag eines Einzeltropfens vorausgesetzt, lässt sich jedoch eine stroboskopische Aufnahmetechnik einsetzen 91,112 .

In der vorliegenden Arbeit wurden zwei Doppelpuls Nd:YAG Laser als Beleuchtungsquelle für jeweils eine Kamera mit Doppelbildaufnahme verwendet. Durch eine synchrone Ansteuerung ließ sich zeitgleich der Einschlag von der Seite und von unten visualisieren. Durch Variation des Zeitpunkts der Aufnahme wurde der Verlauf des Tropfeneinschlags mit einer zeitlichen Genauigkeit aufgelöst, die einer kontinuierlichen Bildrate bis zu 100 Mfps entspricht.

In der Seitenansicht wurde die Ausbreitungsdynamik über den normierten Durchmesser $D / D_{0}$ des sich auf dem Substrat ausbreitenden Tropfens untersucht. Während der Lamella-Phase $(0,1 \lesssim t / \tau \lesssim 1)$ zeigte sich der Zusammenhang $D / D_{0} \sim \sqrt{t / \tau}$ entsprechend $[23]$, wobei die Gültigkeit unabhängig vom Kontaktwinkel des Substrats (hydrophil, hydrophob und superhydrophob) nachgewiesen werden konnte. Unterstützt durch numerische Simulationen wurde gezeigt, dass bei gleichen dimensionslosen Kennzahlen (Reynolds-Zahl, Weber-Zahl) eine Verallgemeinerung der Ausbreitungsdynamik schneller Mikrotropfen und langsamer Millimetertropfen möglich ist. Erst in einer späteren Phase des Einschlags $(t / \tau \gtrsim 1)$ kommt es zu Abweichungen aufgrund der Benetzbarkeit des Substrats. Im Fall des superhydrophoben Substrats wurde nach der Abbremsung eine Rückströmung und anschließend ein partieller Rückprall des Tropfens beobachtet.

Abhängig von der Weber- und Reynolds-Zahl ist die Ausbreitung des Tropfens auf dem Substrat durch die rücktreibende Oberflächenspannung und/oder durch 
die Dissipation aufgrund der Viskosität der Flüssigkeit bestimmt. Es wird ein maximaler Ausbreitungsdurchmesser $D_{\text {max }}$ erreicht. Unter Berücksichtigung einer sich am Rand der Lamella bildenden Wulst und der Dissipation in einer substratnahen Grenzschicht wurde eine bereits für Millimetertropfen semiempirisch gefundene Beschreibung [8] verifiziert. Mit Hilfe der vorliegenden Experimente konnte nun gezeigt werden, dass eine Verallgemeinerung auf Mikrotropfen möglich ist und der Prozess allein durch Weber- und Reynolds-Zahl beschreibbar ist.

Die synchrone Aufnahme des Einschlags von unten ermöglicht es, die zeitliche Entwicklung des Tropfenprofils, also die Dicke des Flüssigkeitsfilms auf dem Substrat, zu bestimmen. Aufgrund der Verwendung von monochromem, kohärentem Laserlicht zur Beleuchtung kommt es zur Bildung von Interferenzstreifen (zwischen dem glatten Substrat und der gewölbten Tropfenoberseite). Aus dem sich ergebenden Interferenzmuster lässt sich die Höhe der Flüssigkeit über dem Substrat mit der gleichen hohen räumlichen und zeitlichen Auflösung wie in den Seitenaufnahmen erreichen. Ein anschließender Vergleich der dreidimensionalen Darstellung mit der tatsächlichen Tropfenform aus der Seitenansicht weist eine sehr hohe Übereinstimmung auf. Da dies auch für die numerische Simulation gilt, zeigt sich auch hier die skalenfreie Beschreibbarkeit mittels dimensionsloser Kennzahlen.

Wichtige weitere Fragestellungen z.B. zum Einschluss von Bläschen unterhalb des Tropfens, zur Skalierung der Wulst am Rande der Lamella, oder auftretender Grenzschichtströmungen lassen sich mit der beschriebenen Methode ebenfalls detailliert untersuchen.

Es kann zusammenfassend gesagt werden, dass durch die diskutierten Ergebnisse bereits existierende Modelle für Millimetertropfen und die verwendete numerische Berechnung ihre Gültigkeit auch für Mikrometertropfen behalten. Die Möglichkeit der Übertragung bereits validierter Methoden auf Mikrotropfen ermöglicht es, auch in diesem experimentell schwer zugänglichen Parameterbereich detaillierte Aussagen über den Einschlagprozess zu treffen. 



\section{Kapitel 3}

\section{Messung der substratnahen Strömungs- geschwindigkeit beim kontinuierlichen Auftreffen von Tropfen auf ein rotierendes Substrat}

\subsection{Motivation}

In den Kapiteln 1 und 2 wurden die Erzeugung eines monodispersen Tropfensprays mit einer hohen Geschwindigkeit beschrieben und der Aufprall einzelner Mikrotropfen auf einem Substrat untersucht. Die Anwendung von Hochgeschwindigkeits-Sprays zur Reinigung von Oberflächen ist u.a. eine Methode in der Halbleiterfertigung [57-59]. Im Hinblick auf diese Reinigungsmethode soll nun die Wirkungsweise dieses Hochgeschwindigkeits-Sprays unter möglichst praxisnahen Bedingungen untersucht werden. Der Reinigungsprozess lässt sich wie folgt vereinfacht beschreiben: Auf ein rotierendes Substrat (z.B. einen Siliziumwafer) wird kontinuierlich ein flüssiges Medium aufgebracht, sodass sich ein geschlossener Flüssigkeitsfilm bildet. Auf diesen Flüssigkeitsfilm erfolgt der Einschlag des Tropfensprays. Die beim Tropfeneinschlag am Substrat hervorgerufenen Kräfte entfernen anhaftende Verunreinigungen (i.d.R. Partikel) und der kontinuierliche Medienfluss transportiert diese anschließend ab. Nach einem Trocknungsprozess liegt (im Idealfall) ein vollständig gereinigter Siliziumwafer vor. Trotz der Verbreitung dieser Methode ist häufig der zugrundeliegende physikalische Prozess des Tropfeneinschlags nicht ausreichend verstanden [57,68,70]. Die naheliegenden, relevanten Parameter wie Tropfengröße und -geschwindigkeit werden durch weitere Parameter wie z.B. die Dicke des Flüssigkeitsfilms, den Einschlagswinkel und die Umdrehungsgeschwindigkeit des Substrats ergänzt. 
Ziel der vorliegenden Untersuchung ist es, die nahe am Substrat auftretende Strömungsgeschwindigkeit und die damit verbundene Wandschubspannung für ein anwendungsbezogenes Beispiel über einen möglichst weiten Parameterbereich zu bestimmen. Es wird angenommen, dass die Wandschubspannung der entscheidende Faktor ist und die Adhäsionskraft der Kontamination übersteigen muss, damit es zu einer Abreinigung kommt. Im Unterschied zu den üblichen polydispersen Sprayverfahren wird im Folgenden mit der vorgestellten monodispersen Tropfenkette gearbeitet.

Die Beschreibung des Versuchsaufbaus und der Messmethode ist in Kap. 3.2 dargestellt. Die erzielten Resultate werden in Kap. 3.3 dargestellt und die Auswirkungen der Prozessparameter auf die zu erwartende Reinigungswirkung diskutiert. Eine Zusammenfassung der Ergebnisse folgt in Kap. 3.4 . 


\subsection{Experimenteller Aufbau}

Es sollen die nahe am Substrat auftretenden Strömungsgeschwindigkeiten während des kontinuierlichen und regelmäßigen Einschlags einer monodispersen Tropfenkette bestimmt werden. In Abb. 3.1 ist eine schematische Darstellung des verwendeten Versuchsaufbaus gegeben. Sie unterteilt sich in: I) die Methode zur Erzeugung des Einschlags einer Tropfenkette auf ein rotierendes Substrat, II) die Methode zur Beleuchtung und Aufnahme des Tropfeneinschlags und III) die Methode zur Messung der Strömungsgeschwindigkeit nahe am Substrat.

I) Als Substrat wurde eine Quarzglasscheibe (,Wafer“) von $300 \mathrm{~mm}$ Durchmesser und $1 \mathrm{~mm}$ Dicke verwendet. Der Wafer wurde in einer kreisrunden, schwingungsgedämpften Halterung fixiert, die das Substrat auf eine Umdrehungsfrequenz von bis zu $\omega / 2 \pi=1500 \mathrm{~min}^{-1}$ bringen kann. Es lässt sich zentral ein kontinuierlicher Medienfluss (hier DI Wasser) aufbringen, welcher über einen Durchflussmesser auf bis zu $Q=1500 \mathrm{ml} \mathrm{min}^{-1}$ eingestellt werden kann. Die nach außen strömende Flüssigkeit wird am Rand des Wafers aufgefangen. In den sich bildenden Flüssigkeitsfilm schlagen kontinuierlich Tropfen fest eingestellter Größe und Geschwindigkeit ein, siehe Kap. 1.

II) Die Beobachtung erfolgt von unten durch das transparente Quarzglas. Der Tropfeneinschlag lässt sich über ein Mikroskop mit langem Arbeitsabstand (InfiniTube Standard, Infinity Photo-Optical GmbH) und ein Mikroskopobjektiv (CFI S Plan Fluor ELWD 20X, Nikon) auf eine Kamera (Sensicam QE double shutter, PCO AG) abbilden, siehe Abb. 3.2. Die Doppelbilder (A und B) der Kamera werden jeweils mit eigenen Beleuchtungsquellen belichtet. (A): Ein LED-Blitz mit einer Dauer von 1 s (Nachbau entsprechend [116, 117|) dient im Gegenlicht zur Visualisierung des Flüssigkeitsfilms und der einschlagenden Kette von Tropfen. (B): Ein frequenzverdoppelter Doppelpuls Nd:YAG Laser (Quanta Ray PIV 400, Spectra Physics) mit einer Wellenlänge von $\lambda=532 \mathrm{~nm}$ dient zur Visualisierung auftretender Strömungsgeschwindigkeiten mittels fluoreszierende Partikel im Wasser. Durch die räumliche Beschränkung des Versuchsaufbaus ist es notwendig, das Laserlicht in einen $6 \mathrm{~m}$ langen Flüssigkeitslichtleiter (Serie 380, Lumatec GmbH) mit einem Durchmesser von $5 \mathrm{~mm}$ einzukoppeln. Anschließend wird das Laserlicht über einen 50:50 Strahlteiler (BSW10R, Thorlabs) unter einem Winkel von $90^{\circ}$ in die Optik eingekoppelt. Der divergente Austritt aus dem Lichtleiter ermöglicht eine homogene Ausleuchtung des gesamten Sichtfeldes durch das Mikroskopobjektiv. Ein Kerbfilter (NF533, Thorlabs) vor dem Kamerachip sperrt das Laserlicht und verhindert damit eine Beschädigung, 
die durch Reflexionen und Rückfokussierungen auftreten könnten. Die gesamte Aufnahmeeinheit lässt sich durch eine präzise xyz-Verschiebeeinheit (Thorlabs) auf die Position des Tropfeneinschlags ausrichten.

III) Um die Strömungsgeschwindigkeit nahe am Substrat zu bestimmen, wurde den Tropfen eine geringe Menge $\biguplus^{1}$ fluoreszierender Partikel (MF-FluoOrange2.5, microParticles $\mathrm{GmbH}$ ) über einen druckfesten Bypass im Anschlusssystem des Flüssigkeitsreservoirs beigemischt. Durch die zwei sowohl in der Zeit als auch in der Intensität unabhängig voneinander steuerbaren Laserblitze lassen sich die mitbewegten Fluoreszenzpartikel anregen und ihre Position zu zwei Zeitpunkten bestimmen. Aus der Zeitdifferenz $\Delta t$ zwischen den Blitzen und der Partikelverschiebung $\Delta s$ lässt sich die lokale Geschwindigkeit bestimmen. Die Bewegungsrichtung ergibt sich aus einer einstellbaren, unterschiedlichen Partikelhelligkeit. Eine geringe Schärfentiefe ermöglicht es, die Bestimmung der Geschwindigkeit auf einen Bereich sehr nahe am Substrat zu beschränken. Im vorliegenden Fall lässt sich dies nach [118 berechnen, und eine konservative Abschätzung ergibt eine Schärfentiefe von

$$
\Delta z=\frac{n \lambda}{N A^{2}}+\frac{n e}{M N A} \lesssim 5 \mu \mathrm{m}
$$

mit dem Brechungsindex $n=1$, der Fluoreszenzwellenlänge $\lambda=584 \mathrm{~nm}$, der numerischen Apertur $N A=0,45$, der Auflösungsgrenze $e=6,5 \mu \mathrm{m}$ und der Vergrößerung $M=20$. Trotz der Bemühung, mechanische Vibrationen im Aufbau zu minimieren, kommt es während der Rotation des Substrats zu Schwankungen ${ }^{2}$ des Abstands zum Objektiv von etwa $0,5 \mathrm{~mm}$. Als Resultat wäre ein Großteil der Aufnahmen nicht fokussiert und damit nicht auswertbar. Mit einem laserbasierten Abstandssensor (LK-G3000, Keyence GmbH) kann zum Zeitpunkt der Aufnahme die laterale Position der Fokusebene im Bezug auf das Substrat bestimmt werden ${ }^{3}$. Entsprechend wird eine Aufnahme nur für den Fall gemacht,

1 Um ein Verstopfen der Düse zu verhindern, befindet sich ein gesinterter Filter vor der eigentlichen Düse. Damit auch dieser nicht verstopft, muss die Partikelgröße und -konzentration möglichst gering gehalten werden, aber gleichzeitig hoch genug für die Messung der Strömungsgeschwindigkeit sein. Die Verwendung von Partikeln mit einem Durchmesser von 2,51 um und eine Verdünnung der Stammlösung von 1:2000 erwies sich hierfür als guter Kompromiss. Für einen $50 \mu \mathrm{m}$ großen Tropfen lässt sich abschätzen, dass im Mittel ein Partikel auf fünf Tropfen kommt. Die große Anzahl der einschlagenden Tropfen führt jedoch zu einer höheren (für die Messung ausreichenden) Partikelkonzentration an der Einschlagsfläche.

2 Auch eine zur Umdrehungsgeschwindigkeit synchronisierte Aufnahme zeigt noch Schwankungen des Abstands zum Objektiv von etwa $0,05 \mathrm{~mm}$.

3 Da der Abstandssensor eine stark reflektierende Fläche für die Messung benötigt, wurde nahe dem Einschlagsort der Tropfen ein durchgehender Farbfilm auf den Wafer aufgetragen. Dadurch ist es möglich, die Abstandsbestimmung nahe am Ort des Einschlags vorzunehmen. 


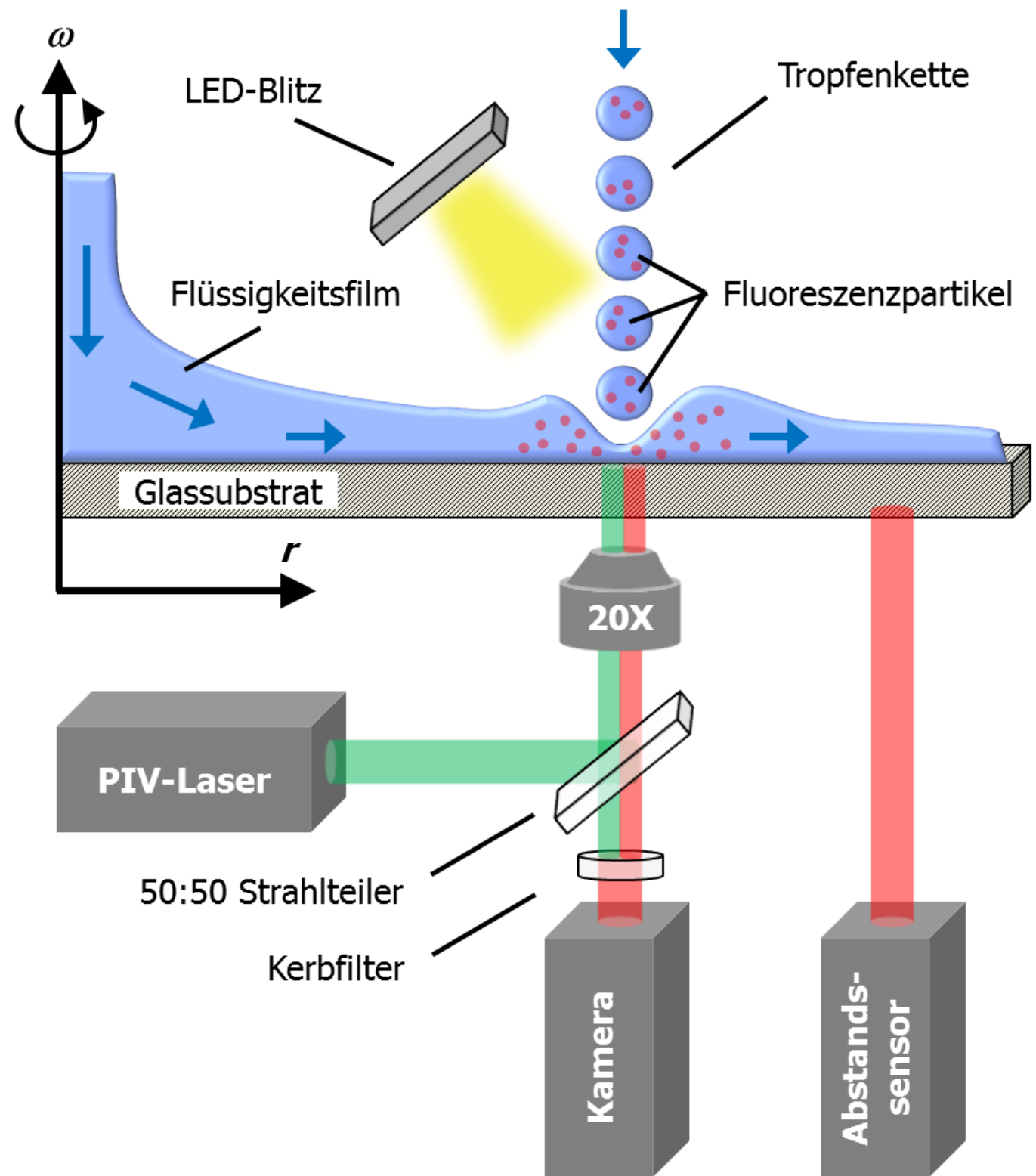

Abbildung 3.1: Schematische Darstellung der Versuchsanordnung zur Untersuchung des kontinuierlichen Tropfeneinschlags auf ein rotierendes Substrat mit einem Flüssigkeitsfilm (nicht maßstabsgetreu). 
in dem sich das Substrat $\pm 2,5 \mu \mathrm{m}$ bezogen auf die Fokusebene befindet. Auf diese Weise sind auf dem Substrat anhaftende Fluoreszenzpartikel in jeder Aufnahme fokussiert und können als Referenz dienen.

Die Methode zur Bestimmung des Betrags und der Richtung der Strömungsgeschwindigkeit basiert auf dem Verfahren der „Particle Image Velocimetry“ (PIV) 94]. Es werden zwei Aufnahmen kurzer Belichtungszeit (hier < $10 \mathrm{~ns}$ ) zeitlich schnell aufeinanderfolgend gemacht. Aus der Korrelation der zwei Aufnahmen ließe sich so das Geschwindigkeitsfeld rekonstruieren. In der vorliegenden Untersuchung wurde, wie bereits erwähnt, bewusst ein abweichendes Vorgehen gewählt, siehe Abb. 3.2. Das erste Bild (A) der Kamera zeigt den Flüssigkeitsfilm und die einschlagenden Tropfen an der Einschlagsstelle. Das zweite Bild (B) wird mit dem PIV-Laser doppelt belichtet. Hierdurch ist eine nahezu gleichzeitige Beobachtung möglich, ob der Einschlag monodisperser Tropfen gegeben ist der Flüssigkeitsfilm an der Einschlagsstelle geformt ist, wo genau das Zentrum des Einschlags ist und wie die räumliche Verteilung der Strömungsgeschwindigkeiten aussieht. Der zeitliche Abstand zwischen den Doppelbildern (A und B) beträgt, durch die Kamera begrenzt, minimal 1 us. Die Doppelbelichtung des zweiten Bildes (B) erlaubt es jedoch, die Zeit zwischen den Blitzen auf bis $\mathrm{zu} 0,1 \mu \mathrm{s} \mathrm{zu}$ reduzieren und ermöglicht somit eine genauere Bestimmung der Strömungsgeschwindigkeit. Nachteil dieser Vorgehensweise ist, dass eine auf Korrelation basierende, automatisierte Methode nicht anwendbar ist. Es zeigte sich, dass nur eine manuelle Identifizierung der Partikelverschiebung zuverlässig und mit hoher Genauigkeit umzusetzen war. Versuche, eine automatisierte Partikelidentifizierung durchzuführen, werden des Weiteren durch das Auftreten von Reflexionen des Laserlichts an der Oberseite des Flüssigkeitsfilms erschwert (trotz Verwendung eines Kerbfilters).

Es wurden vier verschiedene Tropfendurchmesser $D$ (ca. $20 \mu \mathrm{m}, 30 \mu \mathrm{m}, 40 \mu \mathrm{m}$ und $60 \mu \mathrm{m}$ ), drei verschiedene Tropfengeschwindigkeiten $U_{0}$ (ca. $30 \mathrm{~m} \mathrm{~s}^{-1}, 60 \mathrm{~m} \mathrm{~s}^{-1}$ und $\left.90 \mathrm{~m} \mathrm{~s}^{-1}\right)$ und drei verschiedene Medienflüss $\mathrm{s}^{5} Q\left(0 \mathrm{ml} \mathrm{min}^{-1}, 200 \mathrm{ml} \mathrm{min}^{-1}\right.$ und $1500 \mathrm{ml} \mathrm{min}^{-1}$ ) untersucht, um einen möglichst weiten Parameterbereich aus der Anwendung abzudecken. Die weiteren variablen Parameter wurden wie folgt festgelegt: Eine Änderung des Abstands der Düse zum Substrat hat eine zusätzliche Änderung der Einschlagsgeschwindigkeit aufgrund der Luftreibung zur Folge (siehe Kap. 2.3.2). Da es jedoch abhängig von $D, U_{0}$ und $h$ für zu

4 Die Beschränkung durch die räumliche Gegebenheit des Versuchsaufbaus ermöglicht keine Aufnahme der Seitenansicht des Einschlags.

5 Dies entspricht an der Messposition einer Dicke des Flüssigkeitsfilms $h$ von ca. $0 \mu \mathrm{m}, 40 \mu \mathrm{m}$ und $80 \mu \mathrm{m}$, siehe Kap. 3.3.1. 


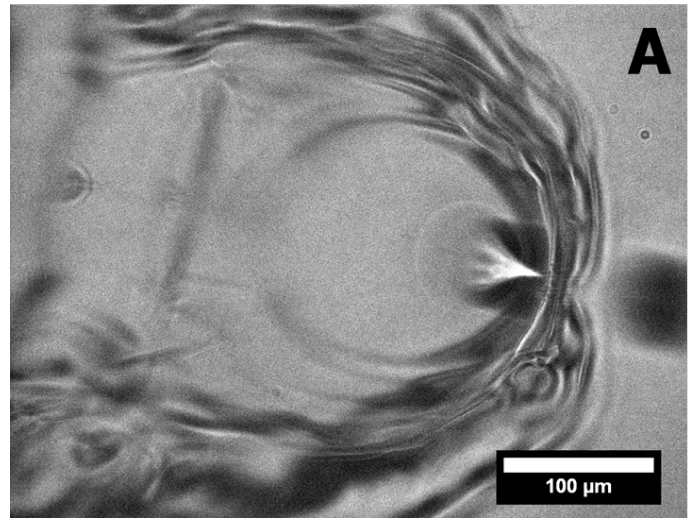

(a)

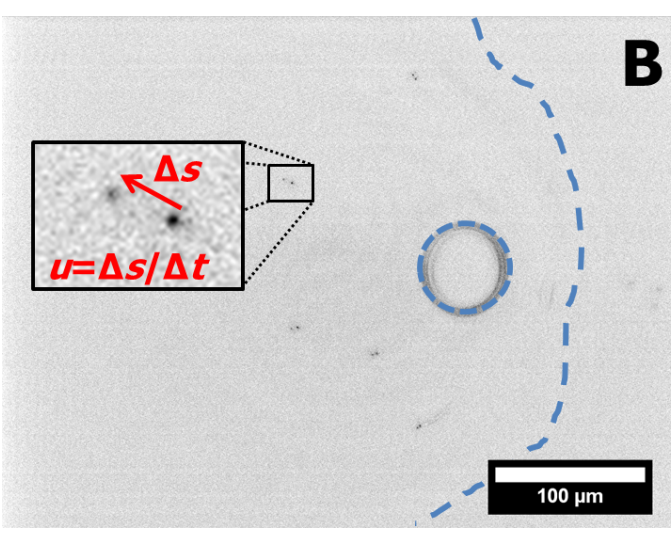

(b)

Abbildung 3.2: Auswertung der Doppelbilder A und B zum Einschlag von Tropfen ( $D=60 \mu \mathrm{m}$ und $\left.U_{0}=34 \mathrm{~m} \mathrm{~s}^{-1}\right)$. (a): Aufnahme der Flüssigkeit und der auftreffenden Tropfen an der Einschlagsfläche (Beleuchtung mit LED-Blitz). (b): Aufnahme der doppelt belichteten Fluoreszenzpartikel (Beleuchtung mit PIV-Laser). Die Partikelverschiebung $\Delta s$ während der Doppelbelichtung $\Delta t$ ist vergrößert dargestellt. Der Ort des Tropfeneinschlags und der Verlauf der Flüssigkeitsfront sind als gestrichelte Linien angedeutet.

geringe Abstände vom Substrat zum Rückspritzen der Flüssigkeit an die Düse kommt [13, 24, was die Funktionsweise der Tropfenerzeugung erheblich beeinträchtigt, wurde ein Abstand von etwa $1,5 \mathrm{~cm}$ gewählt. Hierdurch reduziert sich die eigentliche Einschlagsgeschwindigkeit um etwa $5 \%$. Es kommt ebenfalls zum Rückspritzen, wenn der Tropfeneinschlag senkrecht zum Substrat bzw. entgegen der Drehrichtung des Substrats gerichtet ist. Daher erfolgte der Einschlag schräg entlang der Drehrichtung unter einem Winkel von etwa $53^{\circ}$. Dies hat eine weitere Reduktion der senkrechten Einschlagsgeschwindigkeit von etwa $20 \%$ zur Folge. Da der PIV-Laser am stabilsten bei Repetitionsraten von $10 \mathrm{~Hz}$ arbeitet, wurde eine Umdrehungsfrequenz des Substrats von $600 \mathrm{~min}^{-1}$ gewählt. Der Ort des Tropfeneinschlags wurde auf einen radialen Abstand zum Zentrum des Wafers von $8,5 \mathrm{~cm}$ festgelegt. Aus den letzten beiden genannten Parametern ${ }^{6}$ ergibt sich eine Tangentialgeschwindigkeit des Substrats von etwa $5,3 \mathrm{~m} \mathrm{~s}^{-1}$. In der Auswertung erfolgte eine entsprechende Korrektur der Geschwindigkeitsmessung in Drehrichtung.

6 Beide Parameter sind möglichst nahe der in der Praxis zu erwartenden Tangentialgeschwindigkeit des Substrats gewählt. 


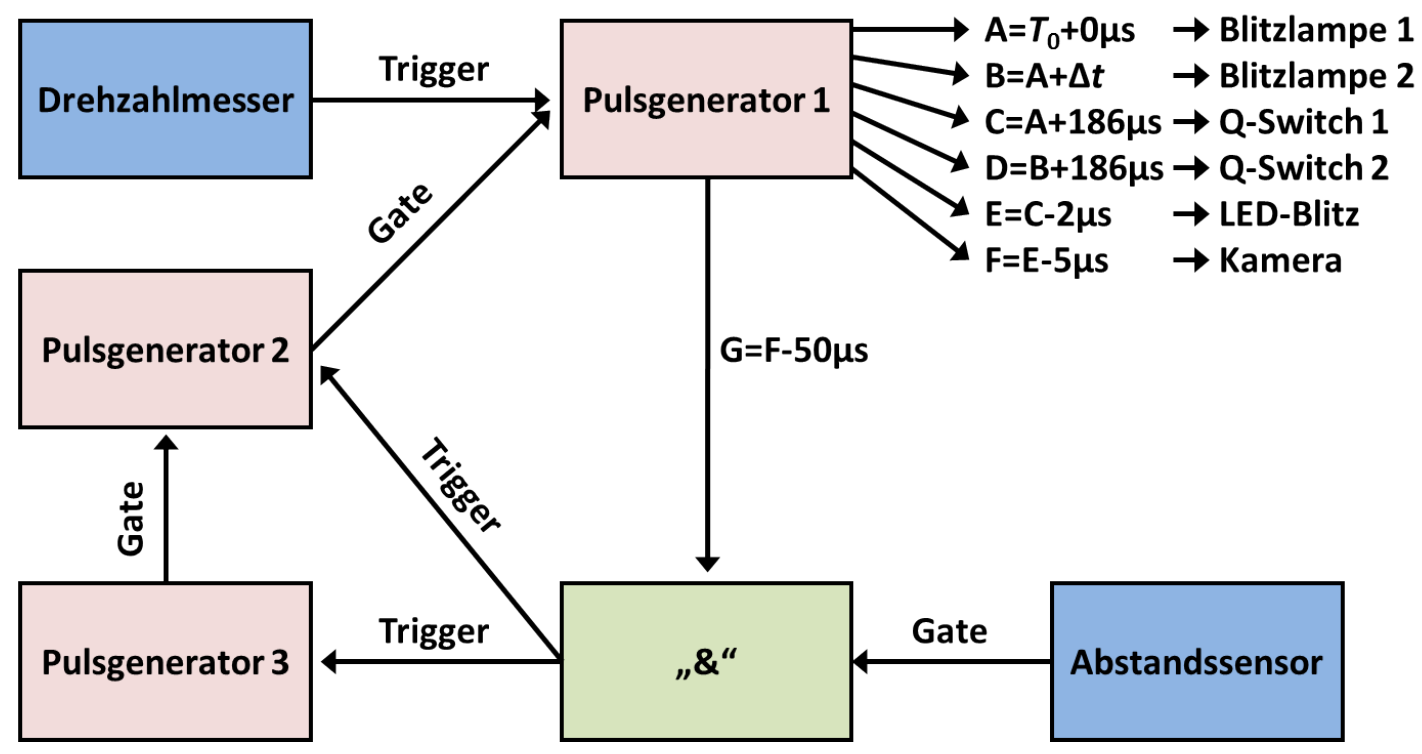

Abbildung 3.3: Zeitliche Ansteuerung und gegenseitige Abhängigkeit der einzelnen Komponenten zur Aufnahme des Tropfeneinschlags (siehe Text).

Es wurden für jedes Experiment zweitausend doppelt belichtete Aufnahmen (B) des Tropfeneinschlags ausgewertet. Abhängig von der Tropfengröße und -geschwindigkeit konnte die Strömungsgeschwindigkeit in Substratnähe bis zu $1500 \mathrm{Mal}$ an verschiedenen Positionen der Einschlagsfläche bestimmt werden. Es wurde in der Versuchsdurchführung bewusst darauf verzichtet, die Geschwindigkeitsmessung zu einer festen Phase des Einschlags durchzuführen. Der Einschlagsprozess derart schneller Mikrotropfen liegt für gewöhnlich im Bereich von etwa 1 us. Insbesondere ist der Beginn des Einschlags sehr transient und es werden währenddessen die höchsten auftretenden Geschwindigkeiten erwartet. Durch die hohe Zahl der aufgenommenen Einschläge kann angenommen werden, dass die sich ändernde Verschiebung zwischen Aufnahme und Einschlagsphase zu einer ausreichenden Überdeckung des Einschlagsprozesses führt. Es wird also sowohl die anfängliche Phase mit hohen Geschwindigkeiten als auch der weitere Verlauf des Einschlags ausgewertet.

Für die Aufnahme des Tropfeneinschlags mit dieser Methode ist ein hohes Maß an zeitlicher Präzision notwendig. Die zeitliche Ansteuerung der Komponenten erfolgte mit Funktions- und Verzögerungsgeneratoren (Modell 575, Berkeley Nucleonics Corporation, $\left.t_{\text {Fehler }}<0,8 \mathrm{~ns}\right)$. Dies ist in Abb. 3.3 gezeigt und wird nachfolgend beschrieben: 
1. Bei jeder Umdrehung des Wafers wird durch einen Drehzahlmesser getriggert vom Pulsgenerator 1 eine neue Aufnahmesequenz gestartet (Kanal A-G). Die Drehzahl beträgt entsprechend der notwendigen Repetitionsrate des PIV-Lasers $10 \mathrm{~Hz}$ bzw. $600 \mathrm{~min}^{-1}$, wodurch die Aufnahme an einer konstanten Position auf dem Wafer möglich ist.

2. Über die Kanäle A-D werden die beiden Belichtungsblitze des PIV-Lasers angesteuert. Hierzu wird erst die jeweilige Blitzlampe und danach der Q-Switch angesteuert, der den Laserblitz auslöst. Das Zeitintervall zwischen den Blitzen $\Delta t$ kann variabel eingestellt werden. Eine zusätzliche Verzögerung $T_{0}$ in Kanal A ermöglicht es, den Anfangszeitpunkt der Sequenz und damit die Position auf dem Wafer zu verändern.

3. Der LED-Blitz (Kanal E) wird $2 \mu$ s vor dem ersten Laserblitz ausgelöst.

4. Die Reaktionszeit der Kamera erfordert eine negative Verzögerung von 5 us (Kanal F) im Bezug auf den LED-Blitz (Kanal E). Die Pulsdauer von 6 us definiert die Belichtungsdauer des ersten Bilds der Kameraaufnahme. Mit einer weiteren Verzögerung von etwa 1 ps erfolgt automatisch die Aufnahme des zweiten Bilds ${ }^{7}$ zum Zeitpunkt der beiden Laserblitze (Kanal C und D).

5. Vor jeder Aufnahme (Kanal F) erfolgt über einen Zeitraum von 50 us (Kanal G) eine Abfrage in einem „\&"Logikelement. Nur wenn während dieser Dauer die Abstandsmessung innerhalb eines definierten Bereiches liegt, werden Pulsgenerator 2 und 3 getriggert ${ }^{8}$. Pulsgenerator 2 aktiviert anschließend über das Gate von Pulsgenerator 1 die Aufnahme (Kanal F). Gleichzeitig wird jedoch das Gate von Pulsgenerator 2 durch Pulsgenerator 3 gesteuert. Die maximale Aufnahmegeschwindigkeit der Kamera für Doppelbilder beträgt $5 \mathrm{~Hz}$. Nach einer erfolgten Aufnahme wird Pulsgenerator 2 und damit eine erneute Aufnahme innerhalb von $0,2 \mathrm{~s}$ durch Pulsgenerator 3 unterbunden.

7 Die Belichtungszeit betrug, durch die Kamera vorgegeben, etwa $100 \mathrm{~ms}$.

8 Während dieses Zeitraums bewegt sich das Substrat etwa $250 \mu \mathrm{m}$. 



\subsection{Auswertung}

In der Halbleiterfertigung werden häufig Tropfensprays zur Reinigung von sensitiven, strukturierten Substraten verwendet $[57-59]$. Die während des Tropfeneinschlags nahe am Substrat auftretenden Strömungsgeschwindigkeiten und damit die Wandschubspannungen stehen in direktem Zusammenhang mit der Abreinigung von Kontaminationen. Der Parameterbereich der vorliegenden Untersuchung wurde hierfür möglichst nahe den Bedingungen in der Praxis gewählt. Aus den Ergebnissen lassen sich so Aussagen über die zu erwartende Reinigungsfähigkeit ableiten. Zuvor wird in Kap. 3.3.1 auf die Eigenschaften des Flüssigkeitsfilms auf dem rotierenden Substrat und in Kap. 3.3.2 auf die Entwicklung des Einschlagskraters eingegangen.

\subsubsection{Flüssigkeitsfilm auf einem rotierenden Substrat}

Durch einen Flüssigkeitszufluss im Zentrum des rotierenden Substrats entsteht ein Flüssigkeitsfilm. Der Tropfeneinschlag erfolgt im Experiment (wie auch in der Praxis) auf diesen Film, dessen Eigenschaften den Aufprall beeinflussen. Die Dicke dieses Films $h$ hängt sowohl vom radialen Abstand $r$, von der Umdrehungsfrequenz $\omega / 2 \pi$ als auch von dem Volumenstrom $Q$ ab 119,120. Kim und Kim 121 beschrieben zur Modellierung des Films eine inkompressible, viskose Flüssigkeit, die mit einem konstanten Volumenstrom zentral auf ein rotierendes Substrat strömt. Sie validierten ihr Modell durch experimentelle Ergebnisse und numerische Berechnungen. Für dünne Filme formulierten sie ein Gleichungssystem zur Beschreibung der gemittelten Strömungsgeschwindigkeit in radialer Richtung $u$ und in azimutaler Richtung $v$

$$
\begin{aligned}
& \frac{\mathrm{d} u}{\mathrm{~d} r}=\frac{\frac{v}{3 u}\left(2+\frac{k_{\mathrm{C}} v}{r}\right)+\frac{5}{18 u}\left(r+\frac{1}{3 r^{2} u F}\right)-\frac{15 r^{2} u^{2}}{2}}{\frac{k_{\mathrm{A}}}{3}-\frac{5}{54 r u^{3} F}} \\
& \frac{\mathrm{d} v}{\mathrm{~d} r}=-\left(\frac{2}{k_{\mathrm{B}}}+v\left(\frac{1}{r}+\frac{45 r^{2} u}{2 k_{\mathrm{B}}}\right)\right)
\end{aligned}
$$

mit den Konstanten $k_{\mathrm{A}}=6 / 5, k_{\mathrm{B}}=17 / 14$ und $k_{\mathrm{C}}=155 / 126$. Als variable Größe geht die Funktion

$$
F=\frac{3 \omega^{2} Q}{2 \pi g \nu}
$$




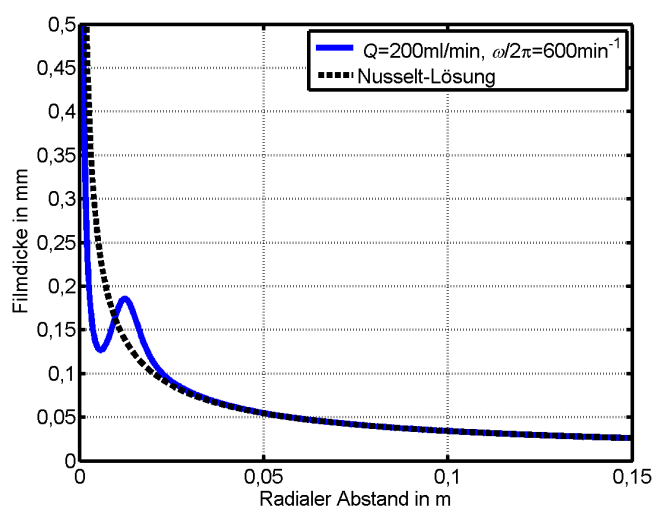

(a)

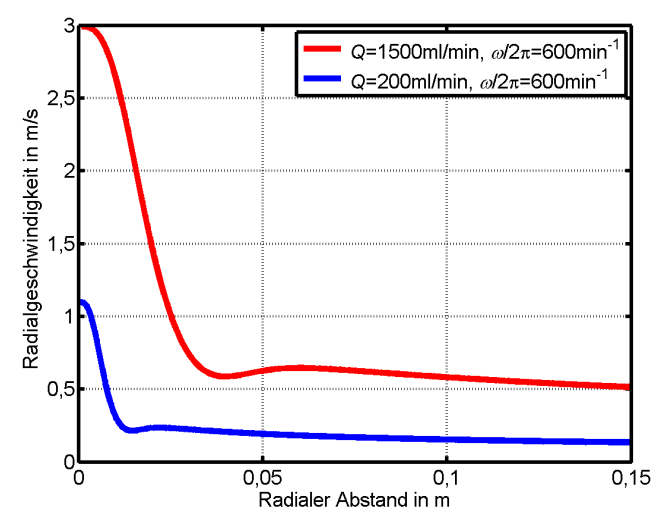

(c)

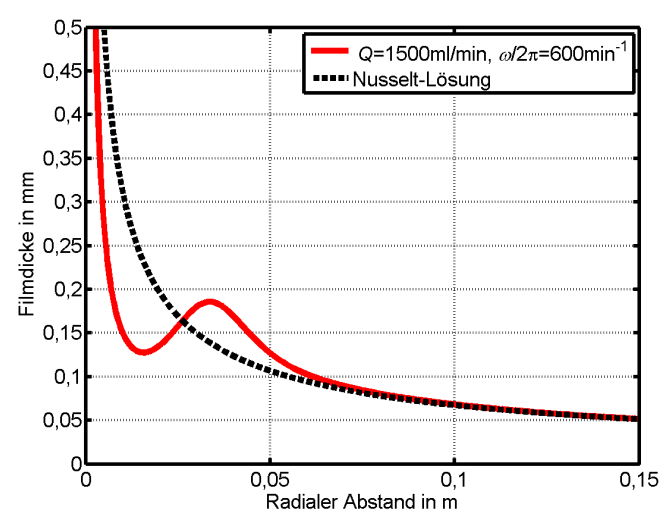

(b)

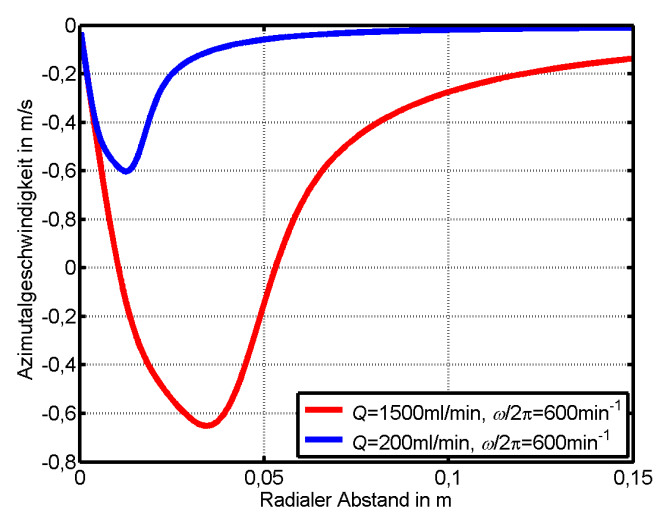

(d)

Abbildung 3.4: Berechnung des Flüssigkeitsfilms in Abhängigkeit vom radialen Abstand. (a): Filmdicke für einen Volumenstrom von $Q=200 \mathrm{ml} \mathrm{min}^{-1}$ und Vergleich mit der Nusselt-Lösung. (b): Filmdicke für einen Volumenstrom von $Q=1500 \mathrm{ml} \mathrm{min}^{-1}$ und Vergleich mit der Nusselt-Lösung. (c): Radialgeschwindigkeit für zwei verschiedene Volumenströme $Q$ und (d) Azimutalgeschwindigkeit für zwei verschiedene Volumenströme $Q$.

in die Gleichungen ein, mit der kinematischen Viskosität der Flüssigkeit $\nu$ und der Erdbeschleunigung $g$. Nach numerischer Integration von Gl. (3.1) und (3.2) lässt sich die Dicke des Flüssigkeitsfilms als Funktion des radialen Abstands mit

$$
h=\frac{1}{3 r u(r)}
$$

berechnen. Für große radiale Abstände lässt sich ebenfalls die Nusselt-Lösung

$$
h_{\text {Nusselt }}=\left(\frac{3 Q \nu}{2 \pi r^{2} \omega^{2}}\right)^{1 / 3}
$$


nach [122 verwenden. In Abb. 3.4a und 3.4b ist die mit dem Modell von Gl. (3.1) und (3.2) berechnete Filmdicke für den im Experiment minimalen Medienzufluss von $200 \mathrm{ml} \mathrm{min}^{-1}$ und maximalen Fluss von $1500 \mathrm{ml} \mathrm{min}^{-1}$ dargestellt. Als Flüssigkeit wurde in beiden Fällen DI-Wasser angenommen. Für kleine radiale Abstände $0 \mathrm{~cm}<r<5 \mathrm{~cm}$ ist ein lokales Ansteigen der Filmdicke zu sehen (hydraulischer Sprung), was jedoch im Experiment aufgrund der geringen Höhenänderung nur schwer zu beobachten war. Außerdem ist die Oberfläche des Flüssigkeitsfilms im Experiment stark durch auftretende Störungen und Verwirbelungen beeinflusst, sodass das berechnete Profil der Filmdicke nur als zeitliche Approximation anzusehen ist. Für große radiale Abstände $r \gtrsim 5 \mathrm{~cm}$ zeigte sich im Versuch eine Abnahme der Störungen auf der Filmoberfläche. Für beide berechneten Zuflussraten ist ab einem radialen Abstand von etwa $5 \mathrm{~cm}$ die Lösung nach [121] nahezu in die Nusselt-Lösung nach [122 übergegangen. Im Experiment wurde ein radialer Abstand von $8,5 \mathrm{~cm}$ für die Beobachtung ausgewählt. Die berechnete Filmdicke an dieser Position beträgt etwa $38 \mu \mathrm{m}$ für den minimalen Medienfluss und etwa $77 \mu \mathrm{m}$ für den maximalen Medienfluss. Da es im Experiment auch an dieser Position zu (wenn auch geringeren) Schwankungen in der Filmdicke kommt, können beide Werte nur als Näherung verstanden werden. Sie sind zur Vereinfachung im Folgenden als $40 \mu \mathrm{m}$ und $80 \mu \mathrm{m}$ bezeichnet.

Durch das Nachaußenströmen der Flüssigkeit und die Rotation des Substrats kommt es abhängig vom radialen Abstand zu Strömungen im Flüssigkeitsfilm, deren Einfluss auf den Tropfeneinschlag abgeschätzt werden muss. In Abb. $3.4 \mathrm{c}$ und $3.4 \mathrm{~d}$ sind die radiale und azimutale Geschwindigkeitskomponente als Funktion des radialen Abstands aufgetragen. Beide Geschwindigkeiten sind für beide Medienflüsse bei einem radialen Abstand von $8,5 \mathrm{~cm}$ vom Betrag $<1 \mathrm{~ms}^{-1}$. Sie sind damit klein gegenüber der Einschlagsgeschwindigkeit der Tropfen. Entsprechend kann der Einfluss der Strömung im Flüssigkeitsfilm vernachlässigt werden.

\subsubsection{Entwicklung des Einschlagskraters}

Beim Einschlag eines Tropfens auf einen Flüssigkeitsfilm der Dicke $h$ bildet sich ein Einschlagskrater $9^{9}$, dessen Größe insbesondere durch die Einschlagsenergie des Tropfens und die Oberflächenspannung der Flüssigkeit bestimmt ist. Die Kraterentwicklung verläuft während einer typischen Zeitspanne $\tau_{\text {Krater }}$. Erfolgt ein weiterer Tropfeneinschlag zu einem Zeitpunkt $t<\tau_{\text {Krater }}$, bildet sich am Boden des ersten Kraters ein zweiter Krater. Für wiederholte Tropfeneinschläge mit ge-

9 Der Einschlagskrater ist durch den unter einem Winkel erfolgenden Tropfeneinschlag und das rotierende Substrat verformt, siehe Abb. 3.2a. 
nügend hoher Frequenz lässt sich ein durchgehend geöffneter Einschlagskrater erzeugen. Ist in diesem Fall die ursprüngliche Filmdicke $h$ klein genug, berührt der Boden des Kraters das Substrat und der Tropfeneinschlag erfolgt auf einem deutlich dünneren (residualen) Flüssigkeitsfilm.

Berberović et al. 25 untersuchten den Tropfeneinschlag auf einen dünnen Flüssigkeitsfilm der Dicke $0,5 \leq h / D \leq 2$. Dies ist vergleichbar mit den Parametern der vorliegenden Untersuchung von $h / D=40 \mu \mathrm{m} / 60 \mu \mathrm{m} \approx 0,7 \mathrm{bis} h / D=$ $80 \mu \mathrm{m} / 20 \mu \mathrm{m}=4$. Der Bereich der Reynolds-Zahl in $[25 \mid$ betrug $300 \lesssim R e \lesssim 8600$ und in der vorliegenden Untersuchung $R e=\rho U_{0} D / \mu \sim 10^{3} \cdot 30 \cdot 2 \cdot 10^{-5} / 10^{-3}=600$ bis $R e=10^{3} \cdot 90 \cdot 6 \cdot 10^{-5} / 10^{-3}=5400$, was ebenfalls vergleichbar ist. Berberović et al. 25 verifizierten ihr analytisches Modell zur Beschreibung der Dynamik des Kraters durch den Vergleich von Experimenten und numerischen Berechnungen. Die typische Zeitskala für den Krater kann mit

$$
\tau_{\text {Krater }} \sim \frac{h}{U_{\text {Krater }}} \sim \frac{2 h}{U_{0}}
$$

abgeschätzt werden und liegt im Bereich von etwa $0,9 \mu \mathrm{s}$ bis $5,3 \mu \mathrm{s}$ für die vorliegende Untersuchung. Die Frequenz der Tropfenerzeugung und damit des Tropfeneinschlags liegt im Bereich von $100 \mathrm{kHz}$ bis $1500 \mathrm{kHz}$. Entsprechend erfolgt in nahezu allen Fällen der Einschlag eines weiteren Tropfens noch während der vorangegangene Einschlagskrater sich ausbildet, womit ein durchgehend geöffneter Krater zu erwarten ist $t^{10}$. Dies konnte auch im Experiment nachvollzogen werden. Zur Bestimmung der residualen Filmdicke $h_{\text {res }}$ lässt sich nach 25 die Abschätzung

$$
h_{\mathrm{res}} \sim \frac{D}{R e^{2 / 5}}
$$

verwenden und es ergeben sich Filmdicken von $\lesssim 1 \mu \mathrm{m}$. Des Weiteren ist noch der Flüssigkeitsstrom durch die Tropfenkette selbst zu berücksichtigen, welcher zu einer Erhöhung $h_{\text {Kette }}$ der Filmdicke am Einschlagsort führt. Unter Verwendung der Kontinuitätsgleichung und dem Ergebnis aus Kap. 3.3.3 kann abgeschätzt werden, dass gilt

$$
h_{\text {Kette }} \sim \frac{a^{2}}{2 D} \cdot \underbrace{\frac{U_{0}}{U}}_{\approx 1} \sim \frac{a}{8}
$$

10 Die azimutale Geschwindigkeit des Substrats an der Beobachtungsposition beträgt etwa $5 \mathrm{~m} \mathrm{~s}^{-1}=5 \mu \mathrm{m} \mu \mathrm{s}^{-1}$. Hieraus ergibt sich eine teilweise bis nahezu vollständige Überlappung der Tropfeneinschlagsgebiete. 
Dabei ist $a$ der Radius ${ }^{11}$ der Düse und ebenfalls der des Flüssigkeitsstrahls. Für den größten (kleinsten) verwendeten Düsendurchmesser ergibt sich so eine zusätzliche Filmdicke von weniger als 1,6 $\mu \mathrm{m}(0,6 \mu \mathrm{m})$.

Mit der letzten Abschätzung lässt sich zusammenfassen, dass der kontinuierliche Tropfeneinschlag über den gesamten Parameterbereich der Untersuchung in einen durchgehend geöffneten Einschlagskrater und auf eine residuale Filmdicke in der Größenordnung $1 \mu \mathrm{m}$ erfolgt.

\subsubsection{Messung der substratnahen Strömungsgeschwindigkeit}

Die Bestimmung der Geschwindigkeit $u$ nahe am Substrat in einem Abstand $z$ ermöglicht einen Rückschluss auf die auftretende Wandschubspannung $\tau_{\text {Wand }}$. Diese Größe beschreibt die zum Substrat parallele Kraft $F_{\|}$, die auf ein Flächenelement $A$ wirkt, und steht in direktem Zusammenhang mit der Reinigungswirkung. Unter der Annahme einer no-slip Randbedingung und eines linearen Geschwindigkeitsprofils berechnet sich die Wandschubspannung näherungsweise als

$$
\tau_{\text {Wand }}=\left.\mu \frac{\partial u}{\partial z}\right|_{z=0} \approx \mu \frac{u(z)}{z} \quad \text {, für kleine } z \text {. }
$$

Dabei ist die Viskosität der Flüssigkeit $\mu$ der Proportionalitätsfaktor, der die Impulsänderung der Flüssigkeit auf das Substrat beschreibt. Etwaige mit einer Kraft $F_{\text {Adh }}$ anhaftende Kontaminationen sind ebenfalls dieser Kraft ausgesetzt, und es kommt zur Reinigung wenn $\tau_{\text {Wand }} \cdot A>F_{\text {Adh }}$.

Mit dem in Kap. 3.2 beschriebenen Versuchsaufbau ist es möglich, die auftretenden Strömungsgeschwindigkeiten beim Tropfeneinschlag auf den Flüssigkeitsfilm sowohl mit hoher räumlicher als auch mit hoher zeitlicher Auflösung zu bestimmen. Gleichzeitig ermöglicht eine geringe Schärfentiefe von weniger als $5 \mu \mathrm{m}$ eine Beschränkung auf substratnahe Messungen ${ }^{12}$. Die Messmethode ermöglicht weiterhin eine Überprüfung der korrekten Funktionsweise der Tropfenerzeugung (entsprechend Kap. 1) kurz vor der Messung der Strömungsgeschwindigkeiten.

Es wurden vier verschiedene Tropfendurchmesser $D$ (ca. $20 \mu \mathrm{m}, 30 \mu \mathrm{m}, 40 \mu \mathrm{m}$ und $60 \mu \mathrm{m}$ ), drei verschiedene Geschwindigkeiten $U_{0}$ (ca. $30 \mathrm{~m} \mathrm{~s}^{-1}, 60 \mathrm{~m} \mathrm{~s}^{-1}$ und $90 \mathrm{~m} \mathrm{~s}^{-1}$ ) und drei verschiedene Dicken des Flüssigkeitsfilms $h$ (ca. $0 \mu \mathrm{m}, 40 \mu \mathrm{m}$ und $80 \mu \mathrm{m})$ untersucht. Der Übersicht halber sind alle Ergebnisse gesondert in

11 Die Tropfenkette wird entsprechend Kap. 1.4 zurück in die Dimensionsgrößen des ursprünglichen Flüssigkeitsstrahls überführt.

12 Dies ist insbesondere für Messungen in Bereichen um das Einschlagsgebiet mit höherer Filmdicke wichtig. 

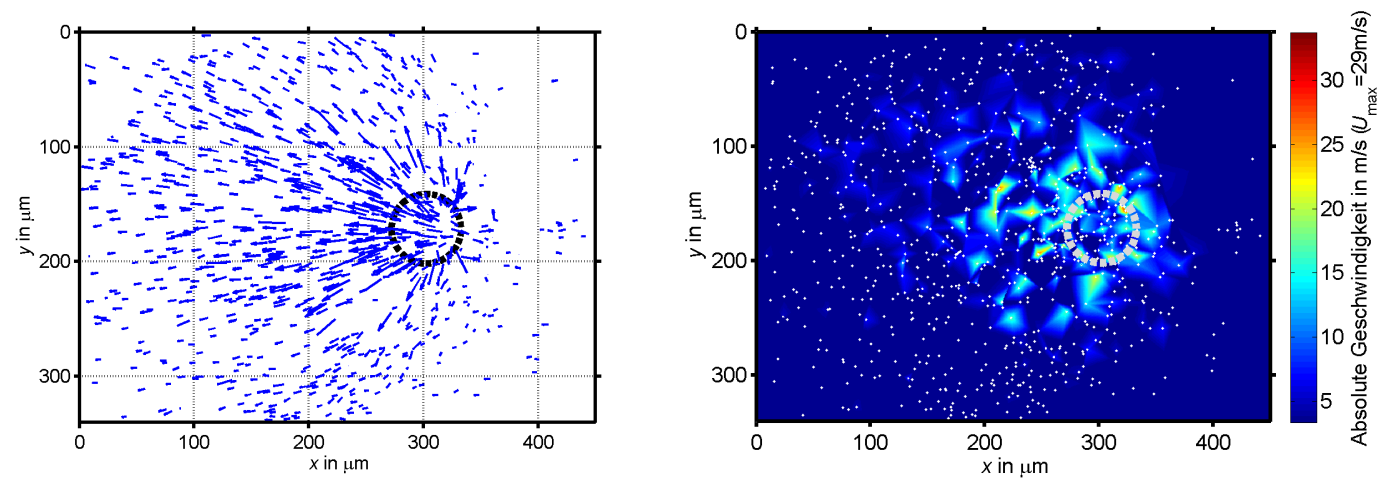

(a) $D=60 \mu \mathrm{m}, U_{0}=34 \mathrm{~m} \mathrm{~s}^{-1}$ und $h=0 \mu \mathrm{m}$
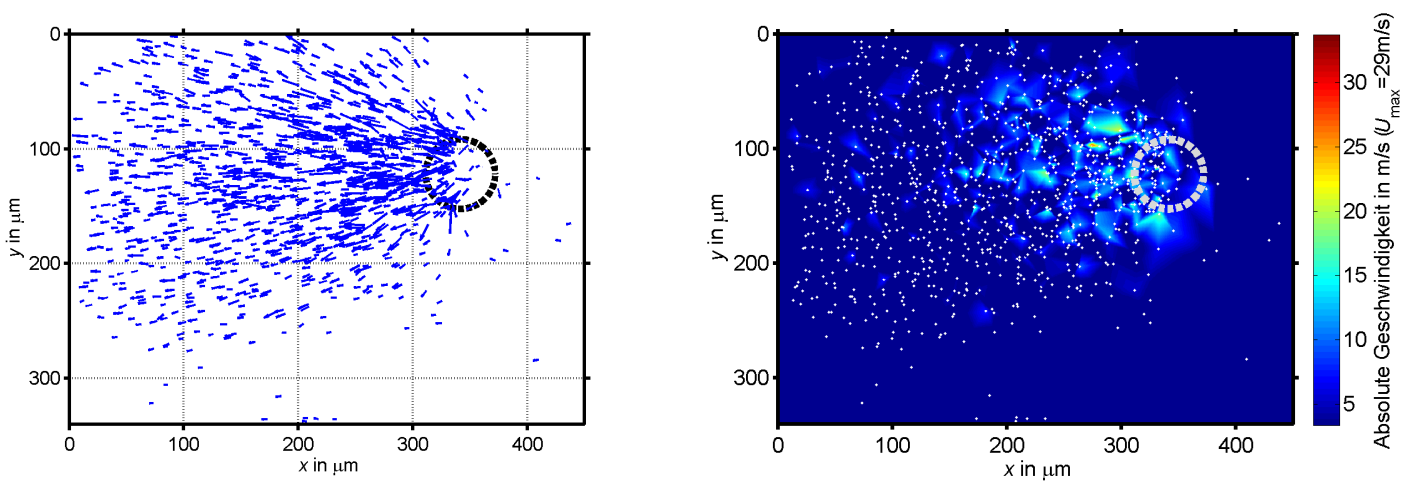

(b) $D=60 \mu \mathrm{m}, U_{0}=34 \mathrm{~m} \mathrm{~s}^{-1}$ und $h=80 \mu \mathrm{m}$

Abbildung 3.5: Geschwindigkeit am Substrat für konstante Tropfengröße $D$ und Tropfengeschwindigkeit $U_{0}$ in Abhängigkeit von der Dicke des Flüssigkeitsfilms $h$. Pfeildarstellung der Strömungsrichtung und -geschwindigkeit (linkes Bild) und Geschwindigkeitsverteilung (rechtes Bild). Weiße Punkte im rechten Bild entsprechen den Messpunkten/Pfeilen im linken Bild. Die Tropfengröße und Einschlagsposition sind durch einen gestrichelten Kreis gegeben.

Anh. F gegeben. Nachfolgend werden ausgewählte Messungen dargestellt und diskutiert. In Abb. 3.5 ist der Einschlag von Tropfen mit einem Durchmesser von $60 \mu \mathrm{m}$ und einer Geschwindigkeit ${ }^{13}$ von $34 \mathrm{~m} \mathrm{~s}^{-1}$ sowohl auf das trockene Substrat (entsprechend $h=0 \mu \mathrm{m}$ ) als auch auf einen Flüssigkeitsfilm der Dicke $h=80 \mu \mathrm{m}$ gezeigt. Für beide Fälle ist jeweils eine Pfeildarstellung der Strömungsrichtung und -geschwindigkeit (linke Teilabbildung) und eine farbkodierte Geschwindigkeitsverteilung (rechte Teilabbildung) gegeben. Die weißen Punkte in der Geschwindigkeitsverteilung markieren die einzelnen Messpunkte und die farbliche

13 Es wurde bereits in Kap. 3.2 darauf eingegangen, dass die tatsächliche vertikale Einschlagsgeschwindigkeit $U$ kleiner als die anfängliche Austrittsgeschwindigkeit aus der Düse $U_{0}$ ist. Da nur letztere im Experiment bestimmt wurde, wird im Folgenden nur $U_{0}$ angegeben. 
Fläche ist zwischen ihnen interpoliert (rot entspricht hoher Geschwindigkeit und blau entspricht geringer Geschwindigkeit). Das Geschwindigkeitsfeld ist deutlich durch den unter einem Winkel auf das rotierende Substrat erfolgenden Einschlag geprägt (siehe Kap. 3.2). Die Bewegungsrichtung des Substrats erfolgt von rechts nach links und wurde in der Auswertung berücksichtigt (d.h. abgezogen). Der Tropfeneinschlag erfolgt in Drehrichtung von rechts kommend. Entsprechend ist eine vermehrt von rechts nach links gerichtete Strömung zu beobachten. Die Einschlagsstelle ist durch einen gestrichelten Kreis in Größe des Tropfendurchmessers markiert, und der Bereich links davon wird nachfolgend als „stromabwärts“ bezeichnet. Im Allgemeinen lässt sich die Fläche der auftretenden Geschwindigkeiten als einen verformten, teils oval bis kegelförmigen Bereich beschreiben. Es zeigt sich insbesondere mit steigender Filmdicke und abnehmender Einschlagsenergie der Tropfen eine starke Begrenzung dieser Fläche durch die auf dem rotierenden Substrat strömende Flüssigkeit. Hohe Geschwindigkeiten treten nahe (jedoch außerhalb) der Einschlagsstelle und insbesondere stromabwärts auf, wo ebenfalls die absolut höchsten Geschwindigkeiten gemessen werden. Im Zentrum der Einschlagsstelle ist ein Bereich geringerer Geschwindigkeiten zu sehen, der durch den sich bildenden Staupunkt begründet ist. Im Falle eines Flüssigkeitsfilms auf dem Substrat kommt es zu einer deutlichen Begrenzung des Bereiches schneller Strömung nahe der Einschlagposition und insbesondere stromaufwärts. Die maximale gemessene Geschwindigkeit beträgt jedoch in beiden Fällen $27 \mathrm{~m} \mathrm{~s}^{-1}$ und liegt in einem Bereich stromabwärts von der Einschlagsstelle. Grund hierfür ist, dass die Einschlagsenergie der Tropfen ausreicht, um den Einschlagskrater permanent zu öffnen, wodurch die sich beim Einschlag ausbreitende Flüssigkeit zu einer Art Abschirmung stromabwärts führt. Gleichzeitig erfolgt so der Einschlag stets auf einen residualen, nur wenige Mikrometer dünnen Film, dessen Dicke nahezu unabhängig für die im Experiment erzeugten Flüssigkeitsfilmdicken auf dem Substrat ist (siehe Kap. 3.3.2). Der hier nicht gezeigte Fall einer intermediären Filmdicke von $h=40 \mu \mathrm{m}$ weist damit übereinstimmend vergleichbare Geschwindigkeitsverteilungen auf. Jedoch kam es für diesen Fall aufgrund der geringen Filmdicke zum gelegentlichen Aufreißen des Flüssigkeitsfilms, sodass nicht zu allen Zeiten ein geschlossener Film gegeben war und der Einschlag somit teilweise auf ein (nahezu) trockenes Substrat erfolgte.

Die Messung der Strömungsgeschwindigkeit ist nicht mit der Phase des Tropfeneinschlags synchronisiert (Begründung siehe Kap. 3.2), und die Geschwindigkeitsverteilung weist eine hohe räumliche Variation auf. Eine nachträgliche Festlegung auf Geschwindigkeiten oberhalb eines bestimmten Wertes ermöglicht eine bessere Visualisierung der tatsächlich maximal auftretenden Geschwindigkeitsverteilung. In Abb. 3.6 ist dieses Vorgehen dargestellt. Indem nur Geschwindigkeiten 


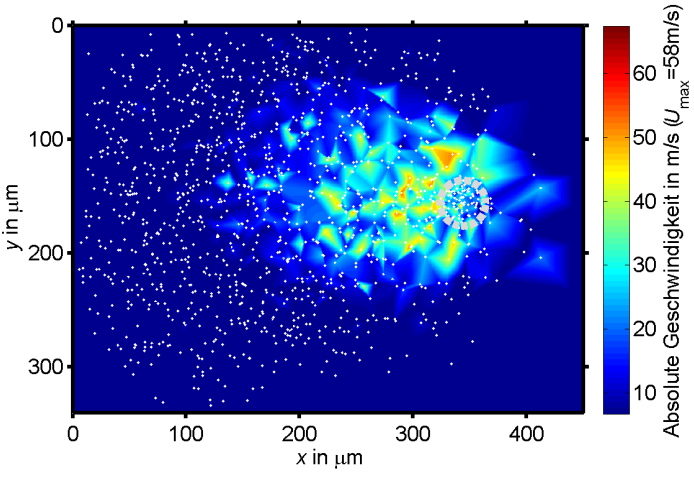

(a)

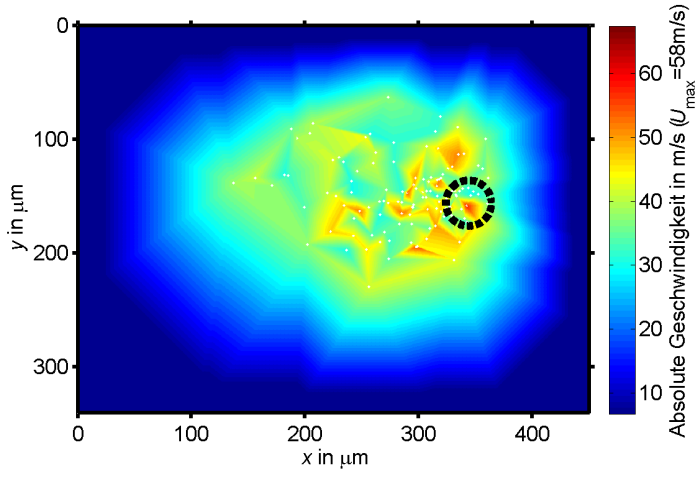

(b)

Abbildung 3.6: Geschwindigkeit am Substrat für $D=40 \mu \mathrm{m}, U_{0}=67 \mathrm{~m} \mathrm{~s}^{-1}$ und $h=40 \mu \mathrm{m}$. (a): Die Darstellung aller Ergebnisse über die Messdauer von 2000 Tropfeneinschlägen zeigt eine hohe Variation der lokalen Geschwindigkeitsverteilung am Substrat. (b): Bei der Darstellung von Geschwindigkeiten $U>30 \mathrm{~m} \mathrm{~s}^{-1}$ lässt sich die maximale während des Tropfeneinschlags zu erwartende Geschwindigkeitsverteilung annähern.

$U>30 \mathrm{~m} \mathrm{~s}^{-1}$ einbezogen werden, ist der tatsächliche Bereich hoher Strömungsgeschwindigkeiten deutlich zu erkennen. Es ist zu erwarten, dass eine Messreihe mit nochmals mehr Datenpunkten zu einer geschlossenen roten Fläche stromabwärts von der Einschlagsfläche führt, die von Bereichen geringerer Geschwindigkeit (erst gelb, dann grün und schließlich blau) umgeben ist. Hier liegen allerdings zu wenig Datenpunkte vor, um ein kontinuierliches Bild zu ergeben.

Die größten Strömungsgeschwindigkeiten sind während der initialen Phase des Tropfeneinschlags zu erwarten, sprich $\tau=D / U_{0}<1$. Im Experiment lag die Zeitspanne zwischen den beiden Laserblitzen im Bereich $\Delta t \lesssim \tau / 2$. Um noch kurzlebigere Strömungen aufzulösen, wurde ein Versuch mit halbierter Zeitspanne $\Delta t \lesssim \tau / 5$ durchgeführt. Es konnten jedoch keine signifikant höheren Geschwindigkeiten gemessen werden. Daraus lässt sich schließen, dass mögliche deutlich höhere Geschwindigkeiten entweder extrem kurzlebig sind mit $\Delta t / \tau \ll 1$ (und sich mit der verwendeten Messmethode nicht aufösen lassen) oder dass sie in höheren Flüssigkeitsschichten über dem Messbereich auftreten und durch Dissipation bereits stark reduziert wurden. Entsprechend ist die maximale im Experiment auftretende Strömungsgeschwindigkeit als für den Reinigungsprozess relevante Größe zu verstehen. Die von den übrigen Prozessparametern abhängige räumliche Ausdehnung/Verteilung dieser Strömungsgeschwindigkeit beeinflusst die „Wirkungsdauer“. Jedoch ist bei einem ausreichend langen Prozess und einer gesamten Überstreichung des Substrats mit dem Tropfenspray zu erwarten, dass 


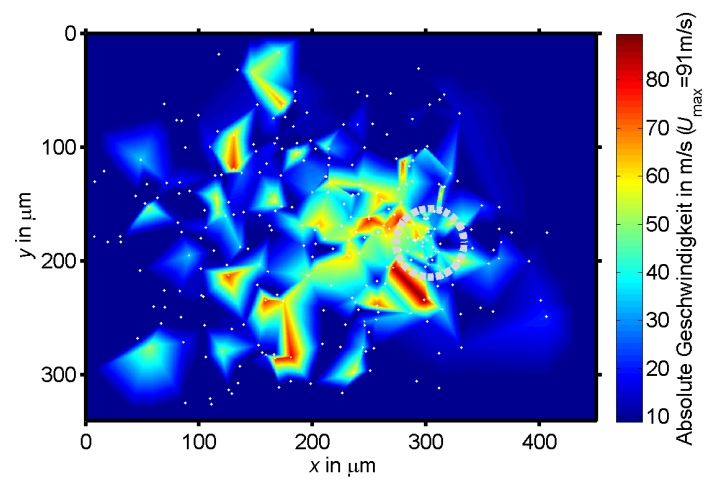

(a) $D=60 \mu \mathrm{m}, U_{0}=90 \mathrm{~m} \mathrm{~s}^{-1}, h=0 \mu \mathrm{m}$

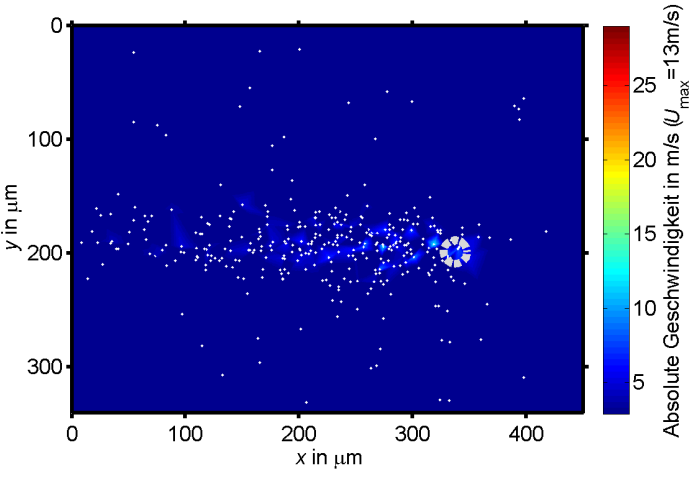

(b) $D=20 \mu \mathrm{m}, U_{0}=29 \mathrm{~m} \mathrm{~s}^{-1}, h=80 \mu \mathrm{m}$

Abbildung 3.7: Vergleich der Geschwindigkeit am Substrat für die höchste und geringste Einschlagsenergie (bezogen auf Tropfengröße und -geschwindigkeit) auf einen Flüssigkeitsfilm mit der minimalen und der maximalen Filmdicke.

die gesamte Substratfläche mindestens dieser Strömungsgeschwindigkeit und damit dieser Wandschubspannung ausgesetzt ist.

Der Einfluss von Tropfengröße, Tropfengeschwindigkeit und Filmdicke auf die erreichbaren maximalen Geschwindigkeiten am Substrat und die Ausdehnung des Bereiches mit hohen Geschwindigkeiten ist in Abb. 3.7 dargestellt. Es wird der Fall maximaler Einschlagsenergie $\left(E_{\max }=4,58 \times 10^{-7} \mathrm{~J}\right)$ von Tropfen mit einem Durchmesser von $60 \mu \mathrm{m}$ und einer Geschwindigkeit von $90 \mathrm{~m} \mathrm{~s}^{-1}$ dem minimaler Einschlagsenergie $\left(E_{\min }=1,76 \times 10^{-9} \mathrm{~J}\right)$ von Tropfen mit einem Durchmesser von $20 \mu \mathrm{m}$ und einer Geschwindigkeit von $29 \mathrm{~m} \mathrm{~s}^{-1}$ gegenübergestellt. Gleichfalls ist der Medienzufluss im ersten Fall gleich Null und im zweiten Fall liegt eine Filmdicke von $80 \mu \mathrm{m}$ vor. Der Vergleich verdeutlicht die starke Reduzierung des Bereiches von hohen Geschwindigkeiten am Substrat (rote Farbe). Im Fall minimaler Einschlagsenergie sind nur direkt am Einschlagsort und nur gering erhöhte Geschwindigkeiten zu messen (grüne Farbe). Über alle Versuche hinweg zeigte sich, dass die Ausdehnung dieses Bereiches sowohl mit der Tropfengröße als auch -geschwindigkeit steigt und mit der Filmdicke abnimmt. Des Weiteren ist ein Einfluss der maximalen Geschwindigkeit im Vergleich zur Tropfengeschwindigkeit zu beobachten: 1) $U_{0}=90 \mathrm{~m} \mathrm{~s}^{-1}$ gegenüber $U_{\max }=91 \mathrm{~m} \mathrm{~s}^{-1}$ und 2) $U_{0}=29 \mathrm{~m} \mathrm{~s}^{-1}$ gegenüber $U_{\max }=13 \mathrm{~m} \mathrm{~s}^{-1}$. Aufgrund der geringeren Einschlagsenergie ist der Einfluss von viskoser Dissipation während des Einschlags und der erhöhten Filmdicke stärker ausgeprägt. Die Ausbildung hoher Strömungsgeschwindigkeiten beim Tropfeneinschlag ist reduziert und es kommt schon auf kürzeren Längenskalen zu einer deutlichen Abbremsung der Strömung. 


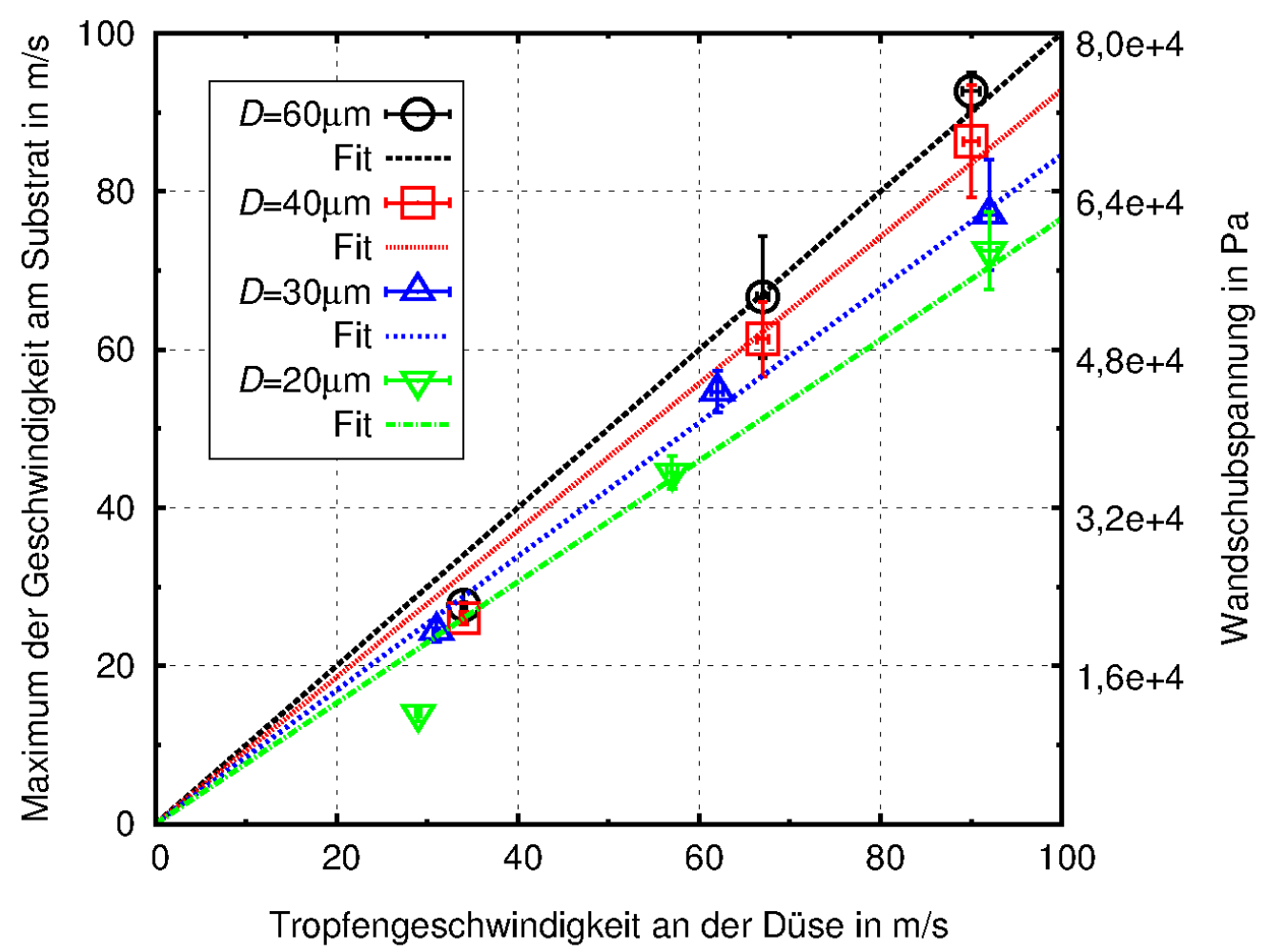

Abbildung 3.8: Überblick des gesamten experimentellen Parameterbereichs aus Tropfengröße und -geschwindigkeit. Das Maximum der gemessenen Geschwindigkeit am Substrat bzw. die genäherte Wandschubspannung wurden als Mittel über die drei Dicken des Flüssigkeitsfilms bestimmt. Ein näherungsweise linearer Anstieg ist zu beobachten (gestrichelte Linien).

In Abb. 3.8 sind die Resultate aller Experimente (siehe Anh. F zusammengefasst dargestellt. Als für die Reinigungswirkung entscheidendste Größe wird die maximale am Substrat auftretende Geschwindigkeit $U_{\max }$ betrachtet ${ }^{14}$. Es wurden jeweils die drei möglichen Filmdicken $h$ zu einem Datenpunkt zusammengefasst, da die Abweichungen nur gering sind und sich kein eindeutiger Zusammenhang zwischen $U_{\max }$ und $h$ finden lässt. Die Fehlerbalken zeigen die Standardabweichung vom Mittelwert. Ein linearer Fit dient zur Veranschaulichung eines näherungsweise linearen Anstiegs von $U_{\max }$ mit der Tropfengeschwindigkeit $U$, wobei $U_{\max }$ näherungsweise von der gleichen Größenordnung wie $U$ ist. In allen Fällen führt ein erhöhter Tropfendurchmesser zu einem größeren $U_{\max }$. Für die minimalste Einschlagsenergie der Tropfen zeigt sich die zuvor beschriebene starke Abbremsung der Flüssigkeitsströmung als deutliche Abweichung.

14 Für die Ausdehnung des Bereiches hoher Geschwindigkeiten am Substrat kann angenommen werden, dass nach entsprechend langer Prozessdauer die gesamte Fläche des Substrats mit der maximalen Strömungsgeschwindigkeit $U_{\max }$ überstrichen wird. 
Ausgehend von der gemessenen Geschwindigkeit lässt sich die zu erwartende Wandschubspannung $\tau_{\text {Wand }}$ mit Gl. (3.3) abschätzen, da $U_{\max }$ als mindestens auftretende Größe und damit als untere Schranke anzusehen ist. Zuvor muss die Höhe der Geschwindigkeitsmessung über dem Substrat $z$ bestimmt werden. Die Schärfentiefe im Experiment betrug $\lesssim 5 \mu \mathrm{m}$ (siehe Kap. 3.2). Durch eine Fokussierung auf eine Ebene etwa $5 \mu \mathrm{m}$ unterhalb des Substrats war es möglich, Partikel direkt am Substrat noch relativ fokussiert abzubilden, jedoch gleichzeitig weiter entferntere Partikel genügend stark zu defokussieren. Als Referenz für die Partikelposition über dem Substrat konnten am Substrat anhaftenden Partikel genommen werden, da sie auf jeder Aufnahme sichtbar waren. Die Annahme des Partikelradius von 1,25 $\mu \mathrm{m}$ als mittlere Höhe über dem Substrat, d.h. $z \approx 1,25 \mu \mathrm{m}$, lässt sich so rechtfertigen. Die korrespondierende Wandschubspannung unter Verwendung von Wasser ist in Abb. 3.8 auf der rechten y-Achse dargestellt. Für die höchste Tropfengeschwindigkeit $\left(\approx 90 \mathrm{~m} \mathrm{~s}^{-1}\right)$ wurden Wandschubspannungen im Bereich $6 \times 10^{4} \mathrm{~Pa}$ bis $8 \times 10^{4} \mathrm{~Pa}$ erreicht.

Bei bekannter Adhäsionskraft und Kontaktfläche einer Kontamination ließe sich mit der Darstellung in Abb. 3.8 nun die für eine Reinigung erforderliche Tropfengröße und -geschwindigkeit abschätzen. 



\subsection{Zusammenfassung}

Der Einsatz von Tropfensprays in der Halbleiterfertigung ist eine weit verbreitete Methode zur Reinigung von strukturierten, sensitiven Oberflächen [57-59]. Der Tropfenaufprall erfolgt meist auf ein rotierendes Substrat (z.B. einen Siliziumwafer), auf dem durch einen zentralen Flüssigkeitszufluss ein geschlossener Flüssigkeitsfilm vorliegt. Die beim Tropfeneinschlag auftretenden sowohl zur Reinigung als auch zur Erosion des Substrats beitragenden Prozesse sind noch nicht vollständig verstanden [57, 69,70|. Die Nachteile [54,55, 68 der gewöhnlich polydispersen Verteilung im Spray bzgl. Tropfengröße und -geschwindigkeit konnten in der vorliegenden Untersuchung umgangen werden, indem ein monodisperses Hochgeschwindigkeits-Spray benutzt wurde (siehe Kap. 1). Die räumlichen Beschränkungen machen eine genaue Untersuchung des Prozesses in der Praxis meist undurchführbar. In der vorliegenden Untersuchung wurde ein Aufbau verwendet, der es ermöglicht, alle praxisrelevanten Prozessparameter einzustellen: Umdrehungsgeschwindigkeit des Substrats, Medienzufluss (und damit die Filmdicke auf dem Substrat) und die Position der Spraydüse. Die Verwendung eines transparenten $300 \mathrm{~mm}$ Quarzglaswafers ermöglichte eine genaue Untersuchung der Einschlagsfläche während des Betriebs. Generell ist zu erwarten, dass die Reinigungswirkung direkt mit hohen, nahe am Substrat auftretenden Strömungen zusammenhängt. Zur Visualisierung der auftretenden Strömungen wurden den Tropfen eine geringe Menge fluoreszierender Partikel beigemischt. Mittels einer PIV-ähnlichen Methode wurden die nahe am Substrat auftretenden Strömungsgeschwindigkeiten während des kontinuierlichen Tropfeneinschlags bestimmt. Gleichzeitig konnte die korrekte Funktionsweise des Tropfensprays bzgl. der Monodispersität überprüft werden ${ }^{15}$. Es wurden vier verschiedene Tropfendurchmesser $D$ (ca. $20 \mu \mathrm{m}, 30 \mu \mathrm{m}, 40 \mu \mathrm{m}$ und $60 \mu \mathrm{m}$ ), drei verschiedene Einschlagsgeschwindigkeiten $U_{0}$ (ca. $30 \mathrm{~m} \mathrm{~s}^{-1}, 60 \mathrm{~m} \mathrm{~s}^{-1}$ und $\left.90 \mathrm{~m} \mathrm{~s}^{-1}\right)$ und drei verschiedene Medienflüsse $Q\left(0 \mathrm{ml} \mathrm{min}^{-1}, 200 \mathrm{ml} \mathrm{min}^{-1}\right.$ und $1500 \mathrm{ml} \mathrm{min}^{-1}$ ) untersucht, um einen möglichst weiten Parameterbereich aus der Praxis abzudecken.

Zur Vermeidung von Rückspritzern an die Düse erwies sich ein Abstand von $1,5 \mathrm{~cm}$ über dem Substrat und ein leicht in Drehrichtung ausgerichteter Einschlagswinkel der Düse als zweckmäßig. Diese Prozessparameter (Düsenabstand und Einschlagswinkel) verändern auch die Aufprallgeschwindigkeit, welche sich aber berechnen lässt (siehe Kap. 1 und 2). In der Praxis sollten sie so gewählt werden, dass eine zuverlässige Funktionsweise des Tropfensprays gewährleistet ist.

15 Durch vereinzeltes Rückspritzen der Flüssigkeit an die Düse kann es zur Ablösung von deutlich größeren Tropfen kommen. 
Variation von Umdrehungsgeschwindigkeit des Wafers oder Position des Tropfensprays haben hauptsächlich eine veränderte tangentiale Relativgeschwindigkeitskomponente des Substrats zur Folge. Da diese in allen Fällen klein gegenüber der Einschlagsgeschwindigkeit der Tropfen war, wurde eine radialer Abstand von $8,5 \mathrm{~cm}$ (entspricht etwa dem halben Substratradius) und eine praxisnahe Drehzahl von $600 \mathrm{~min}^{-1}$ für die Untersuchung gewählt.

Sowohl der kontinuierliche Einschlag der Tropfen (der zeitliche Abstand ist von der Größenordnung $\approx 1 \mu \mathrm{s}$ ) als auch der Medienfluss auf dem Substrat erzeugen einen Flüssigkeitsfilm. Beim Tropfeneinschlag entsteht in selbigem ein Einschlagskrater. Die Zeitskala dieses Kraters liegt über der Einschlagsrate der Tropfen, sodass ein permanent geöffneter Einschlagskrater vorliegt. Der Einschlag der Tropfen erfolgt in allen Fällen auf einen residualen Flüssigkeitsfilm mit einer Dicke von der Größenordnung $1 \mu \mathrm{m}$. Es zeigte sich, dass die maximale am Substrat auftretende Strömungsgeschwindigkeit keine eindeutige Variation mit der nominellen Filmdicke auf dem Substrat aufweist. Jedoch verringerte sich die Ausdehnung des Bereiches hoher Geschwindigkeit nahe der Einschlagsstelle mit höherem Medienfluss auf dem Substrat bzw. mit geringerer Einschlagsenergie (d.h. Tropfengröße und/oder -geschwindigkeit).

Es kann erwartet werden, dass die maximale auf dem Substrat auftretende Strömungsgeschwindigkeit die maximale Reinigungswirkung beschreibt. Aus der Strömungsgeschwindigkeit $U_{\max }$ in einer Höhe $z$ über dem Substrat kann die resultierende Wandschubspannung $\tau_{\text {Wand }}$ abgeschätzt werden. Erst wenn sie größer als die Adhäsionskraft $F_{\text {Adh }}$ bezogen auf ein Flächenelement $A$ der Kontamination ist, würde es zur Reinigung kommen. Es wurde gezeigt, dass $U_{\max }$ näherungsweise proportional zur Einschlagsgeschwindigkeit der Tropfen $U$ ist, wobei $U_{\max }$ leicht mit Tropfendurchmesser steigt, jedoch stets von der Größenordnung $U$ ist.

Bei bekannter Adhäsionskraft $F_{\text {Adh }}$ der Kontamination lässt sich somit die erforderliche Tropfengeschwindigkeit einstellen. Der Medienfluss auf dem Substrat, die Umdrehungsgeschwindigkeit des Substrats und die Tropfengröße haben zunächst nur einen sekundären Einfluss auf die Prozessoptimierung. Sie beeinflussen hauptsächlich die Ausdehnung des Bereiches hoher Geschwindigkeit, jedoch nur geringfügig das Maximum der Geschwindigkeit. Eine längere Prozessdauer sollte hier zu einer gleichmäßigen Überstreichung und schließlich Reinigung des gesamten Substrats führen.

Während des Tropfeneinschlags kommt es jedoch auch zu erosiven Prozessen. Insbesondere wirkt eine hohe Wandschubspannung nicht nur „reinigend“, sondern übt ebenso auch eine Kraft gegen auf dem Substrat befindliche Strukturen aus. Daher ist es notwendig, die Tropfengeschwindigkeit genau auf den jeweiligen 
Anwendungsfall abzustimmen. Die in dieser Untersuchung nicht berücksichtigten Erosionsprozesse z.B. aufgrund transienter Stoßwellen [54, 60, 62, 71, 75 müssen für die zerstörende Wirkung ebenfalls berücksichtigt werden. Jedoch ist sowohl die in der vorliegenden Untersuchung bestimmte Wandschubspannung als auch der Drucksprung durch die Stoßwellen näherungsweise proportional zur Einschlagsgeschwindigkeit. Es bliebe zu untersuchen, inwieweit die Dicke des Flüssigkeitsfilms auf dem Substrat auftretende Stoßwellen dämpfen kann. Aus der jetzigen Sicht scheint es zunächst naheliegend, den Einschlagswinkel zu reduzieren, da so die für den Drucksprung entscheidende senkrechte Geschwindigkeitskomponente reduziert werden kann. Gleichzeitig würde die am Substrat auftretende Strömungsgeschwindigkeit nicht deutlich herabgesetzt werden und nach wie vor von der Größenordnung $U$ bleiben.

Zusammenfassend kann gesagt werden, dass erstmals eine Bestimmung der Wandschubspannung beim Aufprall schneller Mikrotropfen unter anwendungsnahen Bedingungen im Bezug auf Reinigung durchgeführt wurde. Mit den gewonnenen Erkenntnissen lässt sich die Reinigungswirkung abhängig von den Prozessparametern verstehen. In Verbindung mit weiteren Untersuchungen zu auftretenden Erosionsmechanismen ließe sich so der Einsatz von monodispersen Tropfensprays auf eine optimale Effizienz aus maximierter Reinigung und minimierter bis gar keiner Oberflächenschädigung (Erosion) einstellen. Erst wenn die strukturelle Stärke der Strukturen auf dem Substrat die Adhäsionskraft der Kontaminationen unterschreitet, erreicht diese Methode ihre Grenze. Hierbei wird natürlich angenommen, dass die Strömung an der Substratoberfläche auch beim Vorhandensein von Mikro- bis Nanostrukturen zum Fall des glatten Substrats ähnlich bleibt. Auch hierzu müssten in der Zukunft weitere Untersuchungen angestellt werden. 



\section{Resumé und Ausblick}

In vielen modernen Anwendungen (z.B. Tintenstrahldrucker, Kraftstoffeinspritzung oder Reinigungsanwendungen von sensiblen Halbleiteroberflächen) ist ein hohes Maß an Verständnis zum Aufprall von Tropfen notwendig, wenn nicht sogar integraler Bestandteil der Anwendung selbst. Die vorliegende Untersuchung befasste sich mit dem Aufprall von schnellen Mikrotropfen, deren Durchmesser im Bereich $10 \mu \mathrm{m}$ bis $100 \mu \mathrm{m}$ und deren Geschwindigkeit im Bereich $10 \mathrm{~m} \mathrm{~s}^{-1}$ bis $100 \mathrm{~m} \mathrm{~s}^{-1}$ liegt, auf ebene feste Substrate. Die Arbeit teilt sich in drei Themenbereiche auf: 1.) Erzeugung monodisperser Hochgeschwindigkeits-Sprays in Form von Tropfenketten, 2.) Erzeugung und Untersuchung des Aufpralls einzelner Mikrotropfen und 3.) Messung der substratnahen Strömungsgeschwindigkeit beim kontinuierlichen Tropfenaufprall auf ein rotierendes Substrat.

Das Tropfenspray mit genau definierter Tropfengröße und -geschwindigkeit wurde zunächst hergestellt. Die zugrunde liegende Methode basiert auf einem extern angeregten Plateau-Rayleigh-Zerfall. Hierbei zerfällt ein Flüssigkeitsstrahl innerhalb eines gewissen Bereiches von Reynolds-Zahl und Ohnesorge-Zahl in Tropfen, während er von einer Düse aus in Luft übergeht. Dieser Zerfallsprozess lässt sich durch eine externe aufgeprägte Störung steuern, sodass immer eine Tropfengröße (und -geschwindigkeit) als Kette entsteht. In dieser Arbeit konnte die Funktionsweise dieser Methode für verschiedene Flüssigkeiten (DI Wasser, 2-Propanol, Aceton, Ethylenglykol, 1-Hexanol und 1-Butanol) nachgewiesen werden. Außerdem konnte gezeigt werden, dass die Methode eine hohe Genauigkeit bzw. Langzeitstabilität mit Abweichungen von den Zielwerten $<0,5 \%$ aufweist. Eine Begrenzung des homogenen Strahlzerfalls durch den Übergang zum „Zerwellen“, wie durch von Ohnesorge in seinem klassischen Artikel [84] beschrieben, wurde nicht beobachtet, auch nicht bei mehr als zweifachem Überschreiten der berichteten Grenzgeschwindigkeit (hier bis ca. $120 \mathrm{~ms}^{-1}$ ). Mit dieser Methode ist es also möglich, den gesamten Parameterbereich einer typischen Reinigungsanwendung aus der Halbleiterfertigung abzudecken. In solch einer Anwendung ist eine frei wählbare und gleichzeitig sehr genau definierte Tropfengröße und -geschwindigkeit erstrebenswert, um Partikelentfernung ohne Zerstörung sensibler Oberflächenstrukturen zu erreichen. 
Die Untersuchung des Einschlagsprozesses schneller Mikrotropfen ist selbst mit modernen Hochgeschwindigkeitskameras aufgrund der sehr kurzen Zeitskalen $(\lesssim 1 \mu \mathrm{s})$ nur sehr eingeschränkt möglich. Erst eine stroboskopische Aufnahme mit Belichtungszeiten im Bereich von Nanosekunden ermöglicht es, den Einschlag sowohl mit hoher zeitlicher als auch hoher räumlicher Auflösung zu untersuchen. Aufbauend auf der Methode zur Erzeugung eines monodispersen Hochgeschwindigkeits-Sprays wurde ein einzelner Mikrotropfeneinschlag präpariert. Durch die gezielte elektrische Aufladung und Ablenkung eines einzelnen Mikrotropfens ist es möglich, sowohl den Zeitpunkt des Tropfeneinschlags als auch die Tropfengröße und -geschwindigkeit mit hoher Präzision zu reproduzieren. In dieser Arbeit wurde ein Modell entwickelt und experimentell validiert, das unter Berücksichtigung der involvierten Prozesse ermöglicht, sehr genau die Trajektorie der Tropfen zu berechnen. Mit Hilfe des Modells konnte ebenfalls die maximal erreichbare Tropfengeschwindigkeit dieser Methode abgeschätzt werden. Es konnten reproduzierbare Einschläge einzelner Mikrotropfen der Größenordnung $50 \mu \mathrm{m}$ und mit Geschwindigkeiten bis zu $50 \mathrm{~m} \mathrm{~s}^{-1}$ erzeugt und untersucht werden. Die erreichte zeitliche Auflösung betrug bis zu $10 \mathrm{~ns}$, was äquivalent zu einer Aufnahmegeschwindigkeit von $100 \mathrm{Mfps}$ ist. Es konnte gezeigt werden, dass sich bereits validierte Modelle zur Beschreibung langsamerer und größerer Tropfen unter Beibehaltung gleicher dimensionsloser Kennzahlen auf schnelle Mikrotropfen übertragen lassen: Die Dynamik des sich ausbreitenden Tropfens auf dem Substrat und das Erreichen eines maximalen Ausbreitungsdurchmessers bleiben (entsprechend skaliert) gleich. Im vorliegenden Parameterbereich ist daher der Tropfeneinschlag als skalenfrei anzusehen und eine Beschreibung mit dimensionslosen Kennzahlen anwendbar. Des Weiteren wurde eine Methode beschrieben, die es erstmalig mit der gleichen hohen zeitlichen und räumlichen Auflösung ermöglicht, das Tropfenprofil während des Einschlags durch interferometrische Messungen zu bestimmen. Hiermit und mit den zuvor genannten Ergebnissen konnte eine auf dimensionslosen Kennzahlen basierende numerische Simulation validiert werden. Anhand dieser Simulation können weitere Einsichten in den Tropfeneinschlagsprozess erlangt werden, wie z.B. das Geschwindigkeitsfeld innerhalb des Tropfens und damit die auftretende Wandschubspannung oder die Durckverteilung innerhalb des Tropfens und auf dem Substrat.

Sprays mit schnellen Mikrotropfen finden, wie schon erwähnt, Anwendung z.B. in Reinigungsprozessen der Halbleiterfertigung. Der Reinigungsprozess lässt sich vereinfacht wie folgt beschreiben: Auf ein rotierendes Substrat (hier ein transparenter Glaswafer) wird kontinuierlich eine Flüssigkeit (hier 
DI Wasser) aufgebracht, die zur Seite wieder abfließt und so einen geschlossenen Flüssigkeitsfilm bildet. Auf diesen Flüssigkeitsfilm erfolgt der Einschlag des Tropfensprays. Die beim Tropfeneinschlag am Substrat hervorgerufenen Kräfte entfernen anhaftende Verunreinigungen in Form von Partikeln, und der kontinuierliche Medienfluss transportiert diese anschließend ab. Nach einem Trocknungsprozess liegt (im Idealfall) ein vollständig gereinigtes Substrat vor. Die Umstände während eines solchen Prozesses in der Praxis machen eine genaue Untersuchung der Reinigungswirkung häufig unmöglich, sodass die genaue Zusammenwirkung der einzelnen Aspekte noch weitgehend unerforscht ist. In dieser Arbeit wurde ein Aufbau benutzt, der die relevanten Parameter (z.B. Tropfengröße und -geschwindigkeit, Umdrehungsgeschwindigkeit des Substrats, Medienfluss/Filmdicke auf dem Substrat, Düsenabstand und -winkel) einstellen kann. Durch die Verwendung einer der PIV-Technik ähnlichen Methode und Ausnutzung einer geringen Schärfentiefe der Aufnahmeoptik war es möglich, die Strömungsgeschwindigkeit sehr nahe am Substrat zu messen und so die für die Reinigung relevante Wandschubspannung während des Einschlags der monodispersen Tropfenkette zu bestimmen. Durch Variation der Tropfengröße im Bereich $20 \mu \mathrm{m}$ bis $60 \mu \mathrm{m}$, der Tropfengeschwindigkeit $30 \mathrm{~m} \mathrm{~s}^{-1}$ bis $90 \mathrm{~m} \mathrm{~s}^{-1}$ und des Medienflusses im Bereich $0 \mathrm{ml} \mathrm{min}^{-1}$ bis $1500 \mathrm{ml} \mathrm{min}^{-1}$ wurde ein Großteil des in der Praxis relevanten Parameterbereichs abgedeckt. Es zeigte sich, dass die maximal am Substrat auftretende Geschwindigkeit näherungsweise proportional zur und von der gleichen Größenordnung wie die Einschlagsgeschwindigkeit der Tropfen ist. Dies gilt unabhängig von dem verwendeten Medienfluss und damit der Filmdicke auf dem Substrat. Jedoch zeigte sich eine leichte Abnahme der maximalen Strömungsgeschwindigkeit mit kleinerem Tropfendurchmesser. In allen Fällen kommt es zur Bildung eines durchgehend geöffneten Einschlagskraters in der Flüssigkeit auf dem Substrat. Die residuale Filmdicke am Boden dieses Kraters, und damit die tatsächliche für den Tropfeneinschlag vorliegende Filmdicke, betrug in allen Untersuchungen $\sim 1 \mu \mathrm{m}$. Es wurde eine deutliche Abhängigkeit der Ausdehnung des Bereiches hoher auftretender Strömungsgeschwindigkeiten am Substrat von der Einschlagsenergie der Tropfen (bzgl. Größe und Geschwindigkeit) beobachtet. Insbesondere für die kleinsten und langsamsten Tropfen in dieser Arbeit war dieser Bereich sehr stark auf die direkte Nähe zum Einschlagszentrum beschränkt. Es kann jedoch angenommen werden, dass das Maximum der auf dem Substrat auftretenden Strömungsgeschwindigkeit die maximale Reinigungswirkung beschreibt. Eine ausreichend lange Prozessdauer sollte auch bei geringerer Ausdehnung des beeinflussten Gebietes bei einer gleichmäßigen Überstreichung schließlich zur gleichmäßigen Reinigung des gesamten Substrats führen. Bei bekannter Adhäsionskraft und Kontaktfläche der Kontamination 
lässt sich schließlich mit den Ergebnissen dieser Arbeit die für die Reinigung erforderliche Tropfengröße und -geschwindigkeit abschätzen.

Diese Arbeit strebte an, einen Beitrag sowohl zur Erzeugung und zum Verständnis als auch zur Anwendung von schnellen Mikrotropfen zu leisten. Abschließend sollen zwei Beispiele verdeutlichen, wie die gewonnenen Erkenntnisse als Grundlage für die Beantwortung weiterer Fragestellungen genutzt werden können. Zum einen kann es während des Tropfeneinschlags zu erosiven Prozessen durch transiente Prozesse wie Stoßwellen (sowohl in der Flüssigkeit als auch in dem Substrat) kommen. Es bliebe zu untersuchen, von welcher Größenordnung diese für den vorliegenden Parameterbereich sind und wovon sie abhängen bzw. wie sie zu reduzieren wären. Die räumlichen und zeitlichen Skalen würden den Einsatz einer validierten numerischen Simulation empfehlen. Zum anderen wäre es notwendig, die Strömungsgeschwindigkeiten nochmals dichter am Substrat und schließlich auch innerhalb von Strukturen auf dem Substrat zu messen. Als Messmethode ließe sich die sogenannte „Total-Internal-Reflection-Fluorescence Microscopy“ (TIRFM) verwenden [123. Mit ihr ist es möglich, nur die ersten $100 \mathrm{~nm}$ oberhalb des Substrats sichtbar zu machen. Zu dieser Methode wurden Vorversuche gemacht, die hier aber nicht weiter ausgeführt werden. Es wird lediglich in Abb. 3.9 eine Beispielaufnahme gezeigt, welche die Machbarkeit illustrieren soll. Es ist die Aufnahme von mitbewegten, fluoreszierenden Partikeln (Durchmesser $100 \mathrm{~nm}$ ) während eines Mikrotropfeneinschlags zu sehen. Als Grundlage diente die zuvor beschriebene Methode zur reproduzierbaren Erzeugung des Einschlags einzelner, schneller Mikrotropfen. Die Auswertung dieses Beispielbildes wird an dieser Stelle nur angedeutet und zeigt eine mögliche, zukünftige Weiterführung dieser Arbeit. 


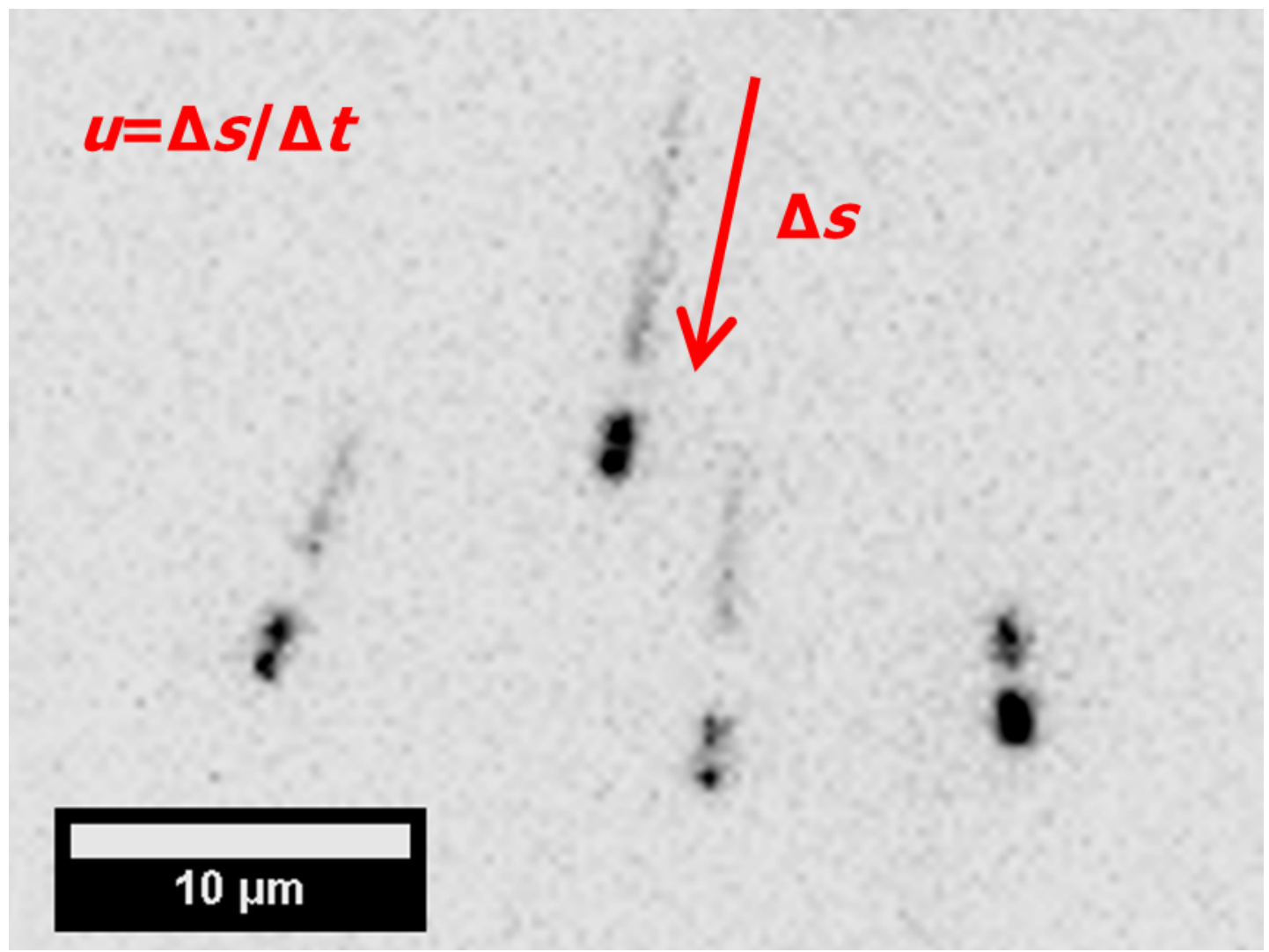

Abbildung 3.9: Vergrößerte Darstellung von mitbewegten Partikeln, die mit TIRFM während des Tropfeneinschlags aufgenommen wurden. Es sind die Bahnen von vier fluoreszierenden Partikeln gezeigt, die drei Mal belichtet wurden (Pulsdauer $2 \mu \mathrm{s}$, Pulsperiode $4 \mu \mathrm{s})$. Aus der Länge der durch die Belichtungsdauer hervorgerufenen Partikelbahn $\Delta s$ lässt sich die mittlere Geschwindigkeit $u$ bestimmen (hier etwa $\left.5 \mathrm{~m} \mathrm{~s}^{-1}\right)$. 



\section{Anhang A \\ Lineare Stabilitätsanalyse eines Flüssigkeitsstrahls}

Der Zerfall eines Flüssigkeitsstrahls in Tropfen soll mit Hilfe der linearen Stabilitätsanalyse durch das Zerfallskriterium eines endlos ausgedehnten flüssigen Zylinders untersucht werden. Entsprechend werden die Zylinderkoordinaten $x, r$ und $\theta$ verwendet, siehe Abb. 1.1 aus Kap. 1. Da zum Zeitpunkt $t=0$ der ungestörte Zylinderradius $R$ entlang der $\mathrm{x}$-Achse konstant ist, kann der Zylinder o.B.d.A. mittels Galilei-Transformation als ruhend angenommen werden. An der Oberfläche des Zylinders $(r=R)$ werden zwei Fälle unterschieden.

I) ungestört:

$$
\begin{aligned}
& p_{0}=p_{\infty}+\frac{\sigma}{R} \\
& \vec{u}_{0}=\text { konst. }=\left(u_{0, \mathrm{x}}, 0,0\right) \equiv(0,0,0) \\
& r_{0}=\text { konst. }=R
\end{aligned}
$$

II) gestört

$$
\begin{aligned}
& p=p_{0}+\tilde{p} \\
& \vec{u}=\vec{u}_{0}+\overrightarrow{\tilde{u}} \\
& r=r_{0}+\tilde{r}
\end{aligned}
$$


Zur Ausführung der linearen Stabilitätsanalyse werden letztere Gleichungen mit den Grundgleichungen der Strömungsmechanik kombiniert:

Euler-Gleichung: Linearisierung und Einsetzen von Gl. A.1 und A.2.

$$
\rho \cdot \partial_{t} \overrightarrow{\vec{u}}=-\vec{\nabla}\left(\left(\mathrm{p}_{\infty}+\frac{\sigma}{R}\right)+\tilde{p}\right)=-\vec{\nabla} \tilde{p}
$$

Kontinuitätsgleichung: Einsetzen von Gl. A.3.

$$
\underbrace{\vec{\nabla} \vec{u}_{0}}_{=0}+\vec{\nabla} \overrightarrow{\tilde{u}}=0
$$

Druck an der Grenzfläche: Benutzung von Gl. A.1 und A.2.

$$
\begin{aligned}
p & =p_{\infty}+\sigma \cdot \vec{\nabla} \vec{n}=p_{0}+\tilde{p}=p_{\infty}+\frac{\sigma}{R}+\tilde{p} \\
\Rightarrow \quad \tilde{p} & =-\sigma \cdot\left(\frac{1}{R}-\vec{\nabla} \vec{n}\right) \\
\Rightarrow \quad \tilde{p} & =-\sigma \cdot\left(\frac{\tilde{r}}{R^{2}}+\frac{\partial^{2} \tilde{r}}{\partial x^{2}}+\frac{1}{R^{2}} \cdot \frac{\partial^{2} \tilde{r}}{\partial \theta^{2}}\right) \quad \text {, bei } r=R \quad .
\end{aligned}
$$

Durch die Tatsache, dass die Oberfläche aus demselben Material wie die Flüssigkeit besteht, wird vorausgesetzt, dass die Teilchen sich mit derselben Geschwindigkeit bewegen. Das bedeutet, dass die radiale Geschwindigkeit gleich der mitbewegten substantiellen zeitlichen Ableitung $u_{\mathrm{r}}=\mathrm{D} r / \mathrm{D} t$ an der Position $r$ ist. Nach Einsetzung von Gl. A.4 ergibt sich

$$
\tilde{u}_{r}=\underbrace{\frac{\mathrm{D} r_{0}}{\mathrm{D} t}}_{\equiv 0}+\frac{\mathrm{D} \tilde{r}}{\mathrm{D} t}=\partial_{t} \tilde{r}+\vec{u} \cdot \vec{\nabla} \tilde{r}=\partial_{t} \tilde{r}
$$

unter Benutzung von $\vec{u} \cdot \vec{\nabla} \tilde{r}=\vec{u}_{0} \cdot \vec{\nabla} \tilde{r}+\overrightarrow{\vec{u}} \cdot \vec{\nabla} \tilde{r}=0$. Der erste Term ist gleich Null, da beide Vektoren senkrecht aufeinander stehen, und der zweite Term ist $\mathrm{zu}$ vernachlässigen, da er quadratisch in der Störung ist.

Im nächsten Schritt wird der Normalmoden-Ansatz verwendet unter der Bedingung, dass alle Störungen sich als Normalmoden der folgenden Form schreiben lassen

$$
\begin{aligned}
& \tilde{p}=\hat{p}(r) \cdot \mathrm{e}^{s t+\mathrm{i} k x+\mathrm{i} n \theta} \\
& \overrightarrow{\vec{u}}=\overrightarrow{\hat{u}}(r) \cdot \mathrm{e}^{s t+\mathrm{i} k x+\mathrm{i} n \theta} \\
& \tilde{r}=\hat{r} \cdot \mathrm{e}^{s t+\mathrm{i} k x+\mathrm{i} n \theta} .
\end{aligned}
$$


Die Divergenz von Gl. A.5 mit Gl. A.6 ergibt für den Druck

$$
\rho \cdot \partial_{t} \underbrace{\vec{\nabla} \overrightarrow{\tilde{u}}}_{=0}=-\Delta \tilde{p}=0
$$

und mit Gl. A.9

$$
\begin{aligned}
0= & \left(i k^{2}\right) \cdot \hat{p} \cdot \mathrm{e}^{s t+\mathrm{i} k x+\mathrm{i} n \theta}+\partial_{r}^{2} \hat{p} \cdot \mathrm{e}^{s t+\mathrm{i} k x+\mathrm{i} n \theta}+\frac{1}{r} \cdot \partial_{r} \hat{p} \cdot \mathrm{e}^{s t+\mathrm{i} k x+\mathrm{i} n \theta} \\
& +\frac{(\mathrm{i} n)^{2}}{r^{2}} \cdot \hat{p} \cdot \mathrm{e}^{s t+\mathrm{i} k x+\mathrm{i} n \theta} \\
= & \partial_{r}^{2} \hat{p}(r)+\frac{1}{r} \cdot \partial_{r} \hat{p}(r)-\left(k^{2}+\frac{n^{2}}{r^{2}}\right) \cdot \hat{p}(r) .
\end{aligned}
$$

Obige Gleichung entspricht einer modifizierten Bessel-Gleichung $n$-ter Ordnung für die Funktion $\hat{p}$ in der Variable $r$ (bzw. $k r$ ). Gelöst wird dies durch Überlagerung der Bessel-Funktion $I_{n}(k r)$ und $K_{n}(k r)$, sodass die allgemeine Lösung die Form

$$
\hat{p}(r)=\mathrm{A} \cdot I_{n}(k r)+\mathrm{B} \cdot K_{n}(k r)
$$

hat. Da es sich um eine symmetrische Funktion in $n$ handelt (o.B.d.A. $n \geq 0$ ) und $\lim _{r \rightarrow 0} K_{n}(k r)=\infty$, lässt sich folgern, dass $B=0$ ist, da der Druck im Inneren einen endlichen Wert annehmen muss.

$$
\Rightarrow \hat{p}(r)=\mathrm{A} \cdot I_{n}(k r)
$$

Für die Geschwindigkeit ergibt sich unter Verwendung von Gl. A.12, A.10 und A.5

$$
\begin{aligned}
\rho \cdot \partial_{t} \overrightarrow{\tilde{u}}=\rho \cdot \overrightarrow{\hat{u}}(r) \cdot s \cdot \mathrm{e}^{s t+\mathrm{i} k x+\mathrm{i} n \theta} & =-\vec{\nabla} \tilde{p} \\
& =-\left(\begin{array}{r}
\partial_{x} \hat{p} \cdot \mathrm{e}^{s t+\mathrm{i} k x+\mathrm{i} n \theta} \\
\partial_{r} \hat{p} \cdot \mathrm{e}^{s t+\mathrm{i} k x+\mathrm{i} n \theta} \\
\frac{1}{r} \cdot \partial_{\theta} \hat{p} \cdot \mathrm{e}^{s t+\mathrm{i} k x+\mathrm{i} n \theta}
\end{array}\right) \\
& =-\left(\begin{array}{r}
\mathrm{A} \cdot I_{n}(k r) \cdot \mathrm{i} k \cdot \mathrm{e}^{s t+\mathrm{i} k x+\mathrm{i} n \theta} \\
k \cdot \mathrm{A} \cdot\left(\partial_{r} I_{n}(k r)\right) \cdot \mathrm{e}^{s t+\mathrm{i} k x+\mathrm{i} n \theta} \\
\frac{1}{r} \cdot \mathrm{A} \cdot I_{n}(k r) \cdot \mathrm{i} n \cdot \mathrm{e}^{s t+\mathrm{i} k x+\mathrm{i} n \theta}
\end{array}\right)
\end{aligned}
$$

Mittels obiger Gleichung ergibt sich die Amplitude der radialen gestörten Geschwindigkeit zu

$$
\hat{u}_{r}(r)=-\frac{\mathrm{A} k}{s \rho} \cdot \partial_{r} I_{n}(k r)
$$


Nun folgt die Berechnung der Eigenwerte von $s$. Hierfür wird zunächst Gl. A.12 in Gl. A.9 und anschließend in Gl. A.7) eingesetzt, unter Verwendung von Gl. (A.11).

$$
\begin{aligned}
\tilde{p}=\quad \mathrm{A} \cdot I_{n}(k R) \cdot \mathrm{e}^{s t+\mathrm{i} k x+\mathrm{i} n \theta}= & -\sigma \cdot\left(\frac{\hat{r}}{R^{2}} \cdot \mathrm{e}^{s t+\mathrm{i} k x+\mathrm{i} n \theta}+(\mathrm{i} k)^{2} \cdot \hat{r} \cdot \mathrm{e}^{s t+\mathrm{i} k x+\mathrm{i} n \theta}\right. \\
& \left.+\frac{1}{R^{2}} \cdot(\mathrm{i} n)^{2} \cdot \hat{r} \cdot \mathrm{e}^{s t+\mathrm{i} k x+\mathrm{i} n \theta}\right) \\
\Rightarrow \quad \mathrm{A} \cdot I_{n}(k R)= & -\frac{\sigma \cdot \hat{r}}{R^{2}} \cdot\left(1-k^{2} R^{2}-n^{2}\right) \\
\Rightarrow \quad \mathrm{A}= & -\frac{\sigma \cdot \hat{r}}{R^{2} I_{n}(k R)} \cdot\left(1-k^{2} R^{2}-n^{2}\right)
\end{aligned}
$$

Anschließend werden Gl. A.11 und A.10 in Gl. A.8 unter Verwendung von Gl. A.13 eingesetzt.

$$
\begin{array}{rlrl} 
& \hat{u}_{r}(r) \cdot \mathrm{e}^{s t+\mathrm{i} k x+\mathrm{i} n \theta}= & \tilde{u}_{r} & =\partial_{t} \tilde{r}=\hat{r} s \cdot \mathrm{e}^{s t+\mathrm{i} k x+\mathrm{i} n \theta} \\
\Rightarrow & -\frac{\mathrm{A} k}{s \rho} \cdot \partial_{r} I_{n}(k R) & =\hat{r} s \\
\Rightarrow & -\frac{\mathrm{A} k R}{\hat{r} \rho R} \cdot \partial_{r} I_{n}(k \mathrm{R}) & =s^{2}
\end{array}
$$

Als letzten Schritt wird Gl. A.14 benutzt und man erhält

$$
\frac{\sigma}{\rho R^{3}} \cdot \frac{(k R) \cdot \partial_{r} I_{n}(k R)}{I_{n}(k R)} \cdot\left(1-(k R)^{2}-n^{2}\right)=s^{2}
$$

Der erste Term $\sigma / \rho R^{3}$ und der zweite Term $(k R) \cdot \partial_{r} I_{n}(k R) / I_{n}(k R)$ sind stets größer als bzw. gleich Nul| für $\forall k R \neq 0$. Daher wird nur noch $\operatorname{sgn}\left(s^{2}\right)=\operatorname{sgn}\left(1-(k R)^{2}-n^{2}\right)$ betrachtet:

Fall 1: $n \geq 1$

Hier gilt $s^{2}<0$ für alle $k R$, was bedeutet, dass $s$ rein imaginär ist bzw. $\operatorname{Re}(s)=0$ ist. Entsprechend sind alle azimutalen Moden stabil und wachsen zeitlich nicht an.

Fall 2: $n=0$ und $|k R| \geq 1$

Auch hier gilt wieder $s^{2}<0$ und $\operatorname{Re}(s)=0$, d.h. für genügend große Wellenzahlen $k$ (oder kleine Wellenlängen) sind alle achsensymmetrischen Moden stabil.

\footnotetext{
1 Im Fall $k=0$ findet keine Anregung (zumindest nicht in $\mathrm{x}$-Richtung) statt.
} 
Fall 3: $n=0$ und $|k R|<1$

Es ist $s^{2}>0$ und hat damit einen Realteil $\operatorname{Re}(s)>0$. Also sind alle achsensymmetrischen Moden mit genügend kleiner Wellenzahl (oder großer Wellenlänge) instabil und wachsen zeitlich exponentiell an.

Somit sind Flüssigkeitsstrahlen aller Durchmesser/Radien (unabhängig von der Dichte und Oberflächenspannung der Flüssigkeit) instabil, sobald die Wellenlänge der Störung größer als der Zylinderumfang ist. 



\section{Anhang B}

\section{Druckabfall entlang eines Rohres mit kreisförmigem Querschnitt}

Nachfolgende Abschätzung dient im Falle einer laminaren Strömung der Bestimmung des zu erwartenden Druckabfalls $\Delta p$ aufgrund der Reibung in den verbindenden kreisrunden Rohr- und Schlauchelementen zwischen dem Druckreservoir (Gasflasche oder Spritzenpumpe) und der Düse [124]:

$$
\Delta p \approx \frac{\rho}{2} \cdot U^{2} \cdot \frac{L}{D} \cdot \frac{64}{R e}
$$

Abbildung B.1 zeigt die berechneten Druckabfälle für einen Rohrdurchmesser von $D=1 \mathrm{~mm}$ und für typische im Experiment verwendete Volumenströme $Q=\pi \rho D^{2} U / 4$. Die Länge $L$ der Verbindungen wurde stets möglichst kurz $(\sim 1 \mathrm{~m})$ gewählt. Es lässt sich erkennen, dass selbst für eine $5 \mathrm{~m}$ lange Verbindung und bei dem höchsten verwendeten Volumenstrom von $3 \mathrm{ml} \mathrm{min}^{-1}$ lediglich ein Druckabfall von etwa 0,1 bar entsteht. Da die Messungenauigkeit des Manometers 0,5 bar betrug, wurde diese Abweichung in der Auswertung nicht berücksichtigt. 


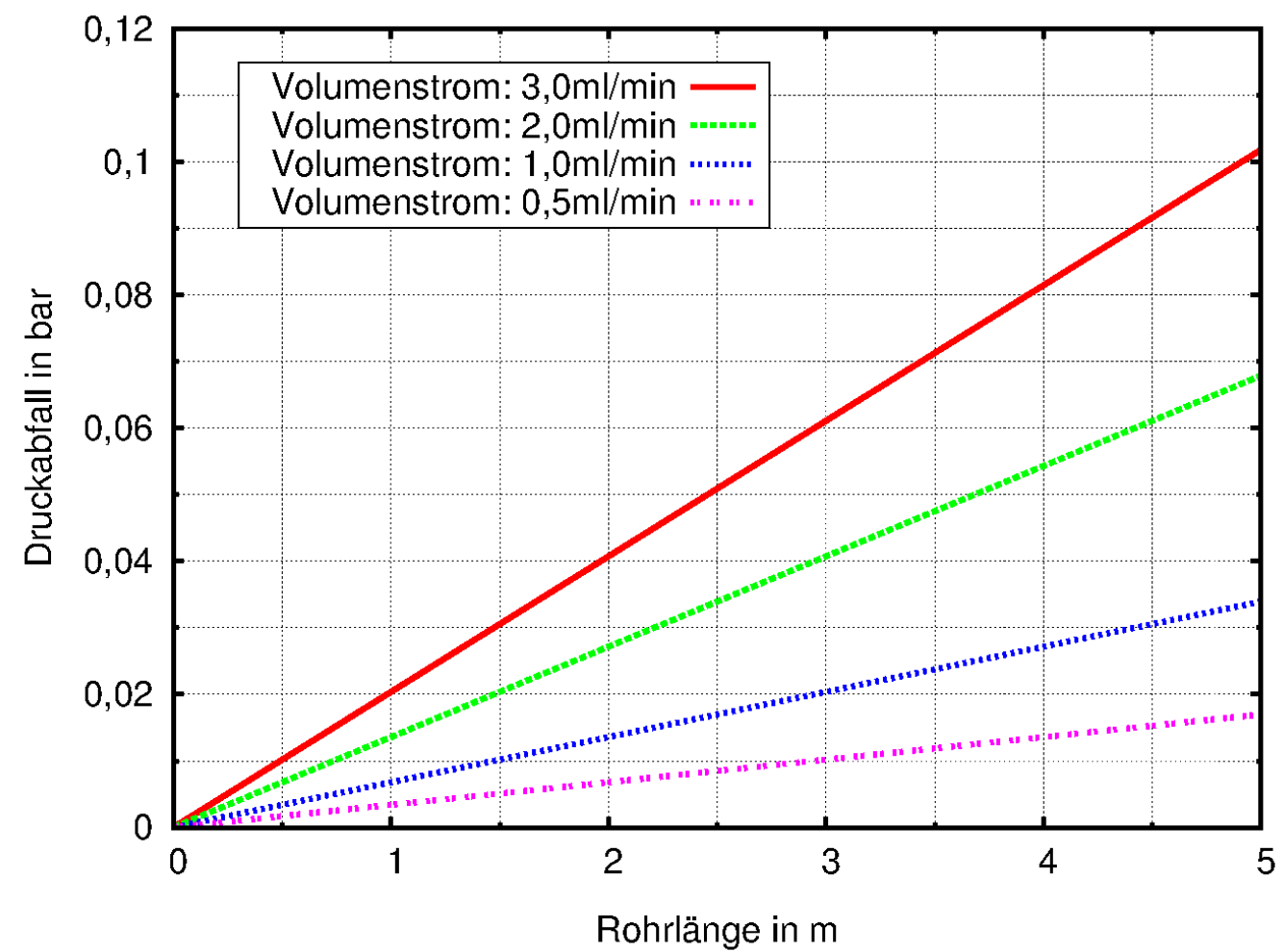

Abbildung B.1: Berechneter Druckabfall für einen Rohrdurchmesser von $1 \mathrm{~mm}$ als Funktion der Rohrlänge und für verschiedene Volumenströme. 


\section{Anhang C \\ Impedanz des piezokeramischen Schallwandlers}

Wird der piezokeramische Schallwandler bei einer Resonanzfrequenz (siehe Abb. C.1) betrieben, so wird ein Maximum der elektrischen Energie in mechanische Schwingungen umgewandelt. Entsprechend ist es möglich, schon mit geringen Amplituden der verwendeten Wechselspannung eine ausreichend starke mechanische Störung auf den Flüssigkeitsstrahl auszuüben, sodass der Zerfall in monodisperse Tropfen realisiert wird.

Es kann jedoch aufgrund der schmalen Frequenzbereiche der Resonanzen bei Variation der Anregungsfrequenz schnell zu einer Überhitzung $\left(>100{ }^{\circ} \mathrm{C}\right.$ in wenigen Sekunden) des Schallwandlers kommen. Die außerhalb der Resonanz notwendige Spannung zur Erzeugung monodisperser Tropfen kann unter Umständen im Resonanzfall zu derart starken inneren mechanischen Verlusten im Schallwandler führen, dass er zerstört wird.

In Abb. C.1 sind der Verlauf der Impedanz und Phase sowohl für den Schallwandler allein als auch im verbauten Zustand an der befüllten Düse abgebildet. Die zuvor gut erkennbaren Resonanzen sind im verbauten Fall stark verbreitert und weniger deutlich. Dennoch ist im Bereich der Resonanz eine starke Erhitzung zu beobachten.

Dies führt zu zwei Überlegungen für eine praktische Implementierung. Erstens: Ist der Betrieb nahe einer Resonanz notwendig, so ist eine zusätzliche Kühlung z.B. durch Luftzirkulation oder durch Verwendung entsprechender Kühlkörper an der Düse unumgänglich. Zweitens: Es sind bei variabler Anregungsfrequenz solche Frequenzbereiche zu bevorzugen, die eine nur gering schwankende Impedanz aufweisen, z.B. ab etwa $1200 \mathrm{kHz}$ für den konkreten Fall in Abb. C.1b. 


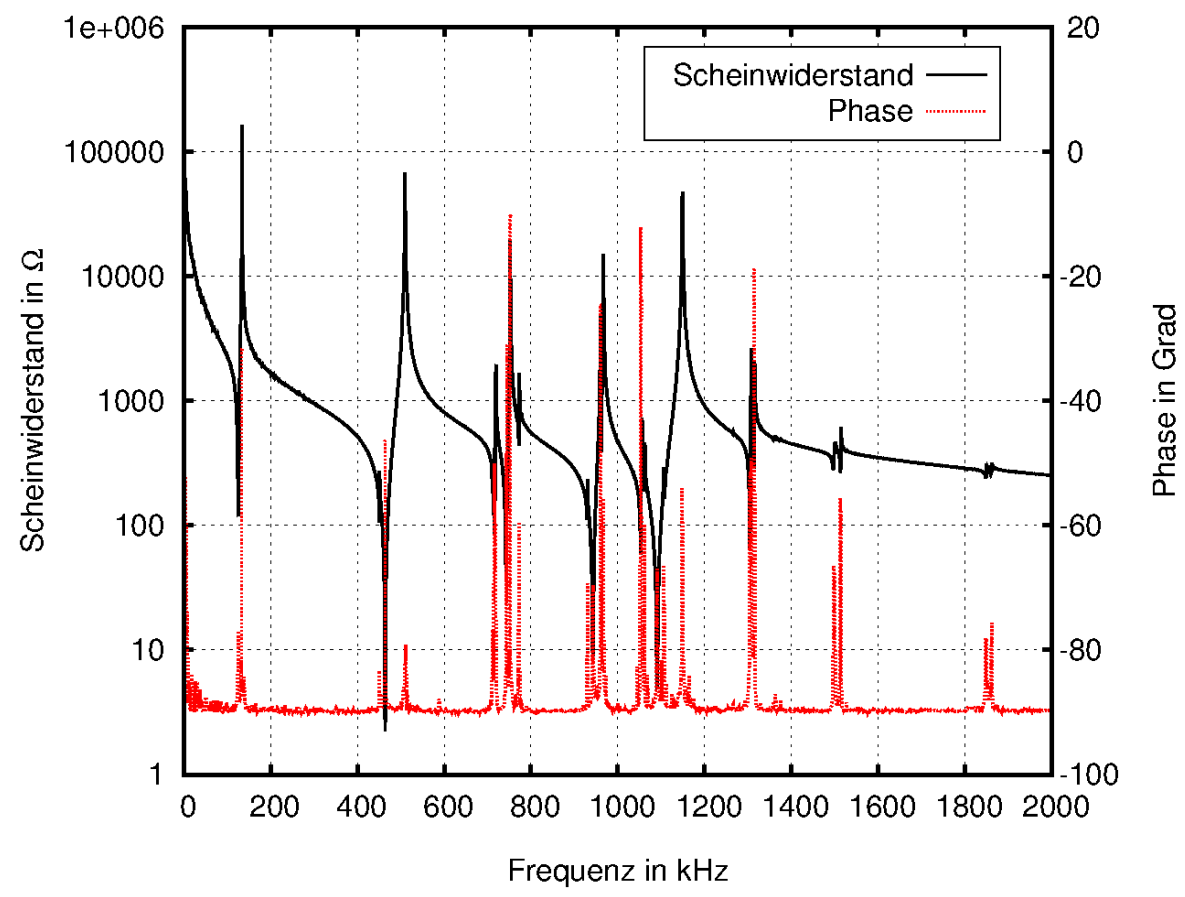

(a)

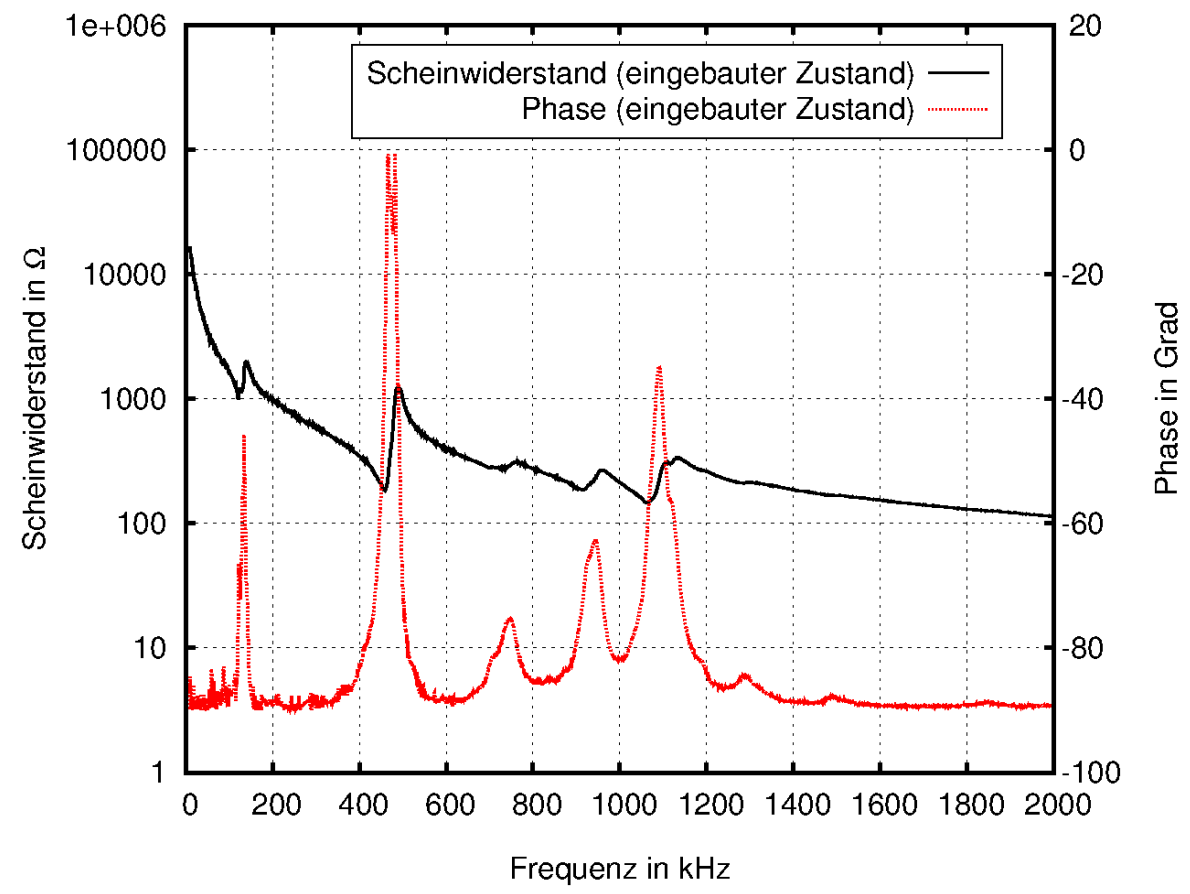

(b)

Abbildung C.1: Gemessener Scheinwiderstand und Phase am verwendeten piezokeramischen Schallwandler über den verwendeten Frequenzbereich. Die deutlich sichtbaren Resonanzen für den unverbauten Zustand (a) sind im eingebauten Zustand (b) deutlich weniger stark ausgeprägt. 


\section{Anhang D \\ Einfluss der Düsengeometrie auf die Austrittsgeschwindigkeit und die Fitparameter $C_{1}$ und $C_{2}$}

Zur Veranschaulichung des Einflusses der Geometrie auf das innere Strömungsverhalten in der Düse und damit auf die Fitparameter $C_{1}$ und $C_{2}$ wurden mit Hilfe von Finite Elemente Simulationen (Laminar Flow Module Single Phase,COMSOL) verschiedene Düsengeometrien bei gleichem Düsendurchmesser berechnet. In Abb. D.1 werden vier Designs mit den experimentellen Ergebnissen der $20 \mu \mathrm{m}$ Edelstahldüse (siehe Kap. 1.3.1) verglichen:

1. „Kegel“: Ausgehend von der $2 \mathrm{~mm}$ messenden Bohrung auf der Rückseite der Edelstahlplatte wird unter konstantem Winkel bis auf ihre Vorderseite (durch eine Dicke von $2 \mathrm{~mm}$ ) gebohrt, sodass der Kegeldurchmesser auf der Vorderseite gerade $20 \mu \mathrm{m}$ beträgt.

2. „Platte“: Ausgehend von der $2 \mathrm{~mm}$ messenden Bohrung auf der Rückseite der Edelstahlplatte wird unter konstantem Winkel bis auf $0,1 \mu \mathrm{m}$ vor Durchbruch auf ihre Vorderseite gebohrt, sodass der Kegeldurchmesser an dieser Stelle gerade $400 \mu \mathrm{m}$ beträgt. In diese dünne Membran ist mittig eine Öffnung von $20 \mu \mathrm{m}$ gebohrt.

3. „Rohr“: Ausgehend von der $2 \mathrm{~mm}$ messenden Bohrung auf der Rückseite der Edelstahlplatte wird unter konstantem Winkel bis auf $50 \mu \mathrm{m}$ vor Durchbruch auf ihre Vorderseite gebohrt, sodass der Kegeldurchmesser an dieser Stelle gerade $110 \mu \mathrm{m}$ beträgt. Anschließend folgt eine halbkugel-ähnliche Bohrung bis auf $5 \mu \mathrm{m}$ vor Durchbruch, an die sich ein Rohr von $5 \mu \mathrm{m}$ Länge und $20 \mu \mathrm{m}$ Durchmesser anschließt.

4. „Rohr*“: Dieses Design ist gleich dem letztgenannten, jedoch sind „Defekte“ und „Unebenheiten“ hinzugefügt, womit es von den Abmessungen und der Form her am besten die eigentliche Geometrie der Edelstahldüse widerspiegelt. 


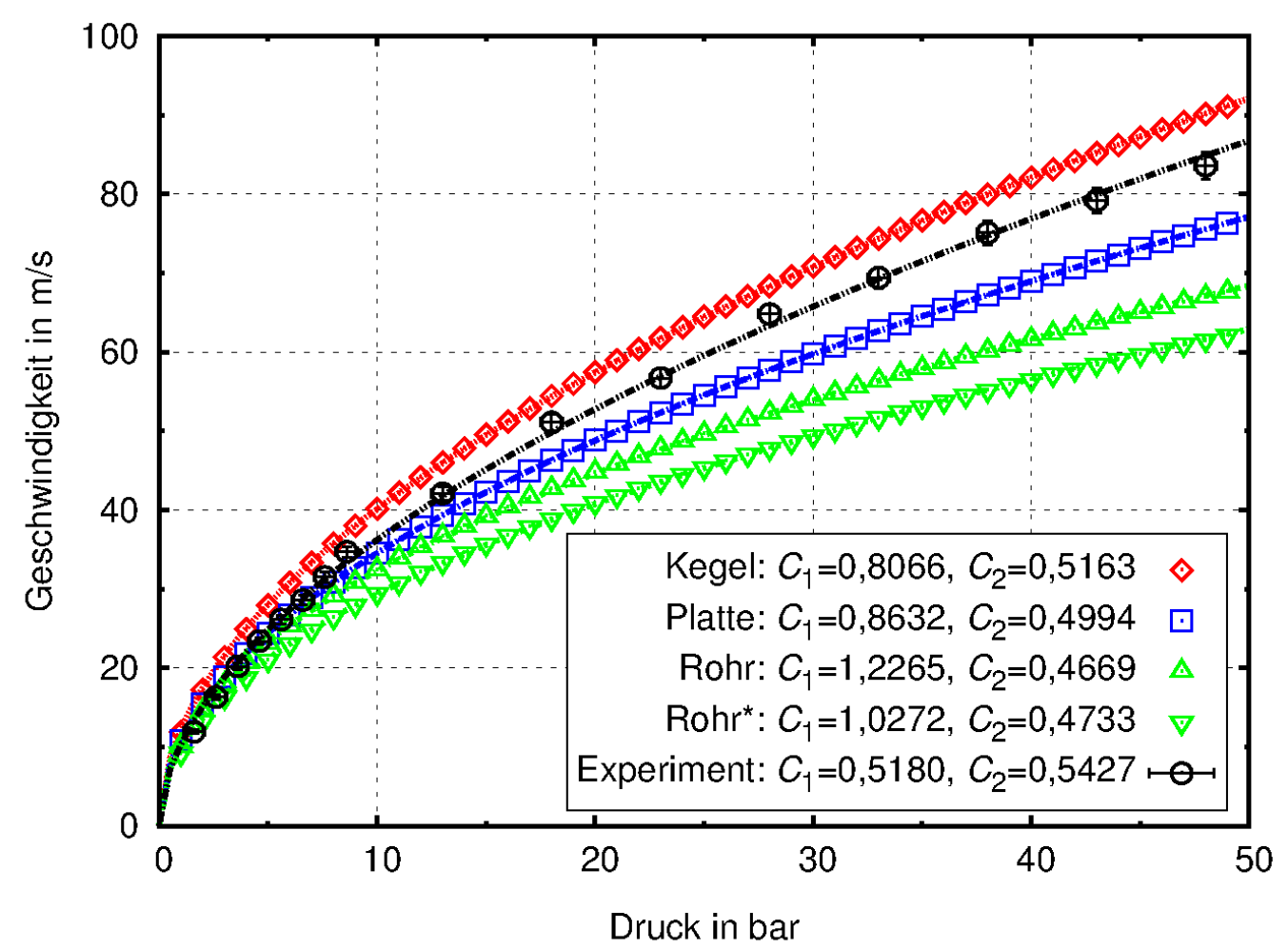

Abbildung D.1: Mittlere Geschwindigkeit über den Düsenquerschnitt für verschiedene Düsengeometrien im Vergleich zum Experiment mit der $20 \mu \mathrm{m}$ Edelstahldüse. In Abhängigkeit vom Druck wurden die Fitparameter $C_{1}$ und $C_{2}$ für jede Düse bestimmt. Die reale Edelstahldüse ist zwischen den Designs „Kegel“ und „Platte“ einzuordnen.

Im Vergleich der Simulationen mit den Messergebnissen der $20 \mu \mathrm{m}$ Edelstahldüse zeigt sich zwar ein vergleichbarer Verlauf, jedoch weichen die berechneten Fitparameter $C_{1}$ und $C_{2}$ und damit deren Kurven teils erheblich ab. Ausgehend von den Berechnungen ist die Edelstahldüse zwischen „Kegel“ und „Platte“ einzuordnen, weist aber die stärkste Abweichung zu dem vermeintlich passenderen Design „Rohr*“ auf. Damit ist diese Herangehensweise nicht für eine hinreichend genaue Beschreibung des Strömungsverhaltens in der Düse geeignet (zumindest nicht ohne substantielle Modifikationen im Programmcode).

Dennoch lässt sich in den vier Kurven der Simulationen der Einfluss einer sich ändernden Geometrie gut erkennen. Eine glatte kegelförmige Düse (Abb. D.2a) ermöglicht einen möglichst laminaren und relativ langsam beschleunigten Durchfluss, ohne hohe Drucksprünge aufgrund einer sich räumlich schnell ändernden Geometrie aufzuweisen. Im Falle einer dünnen Membran (Abb. D.2b lässt sich vermuten, dass die auf ein Minimum beschränkte Kontaktfläche einer schnellen 


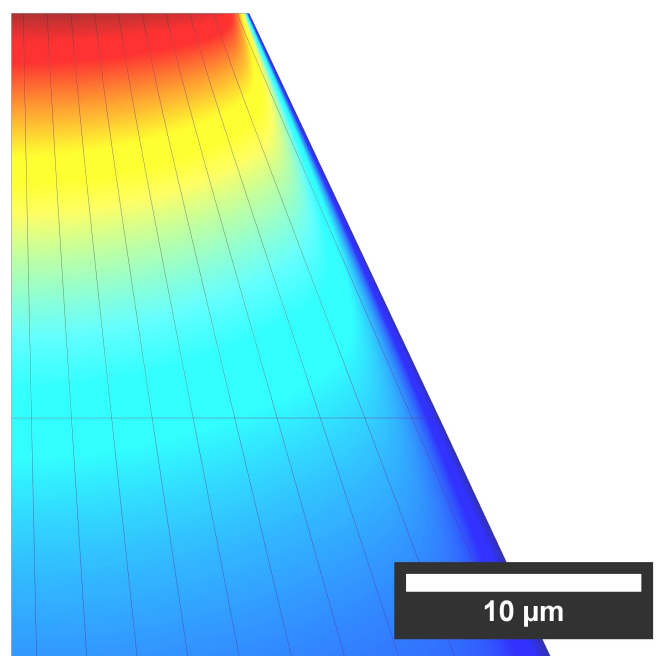

(a) „Kegel“

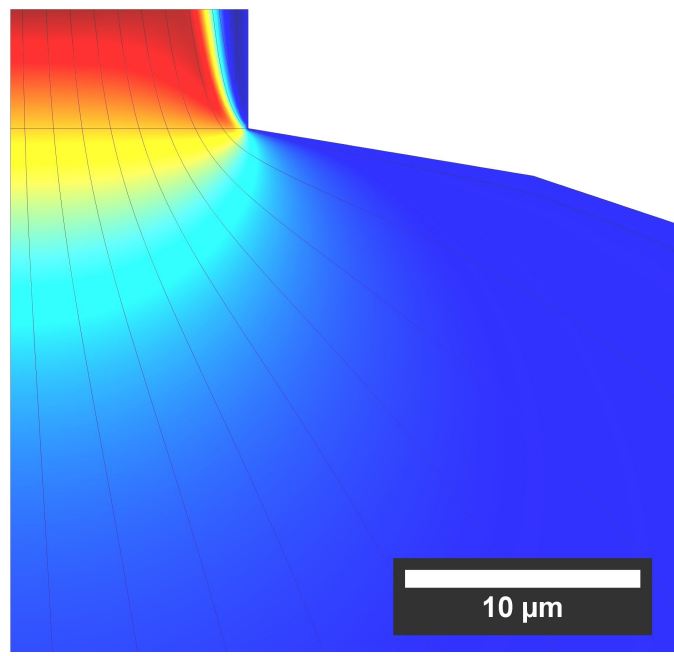

(c) ,Rohr"

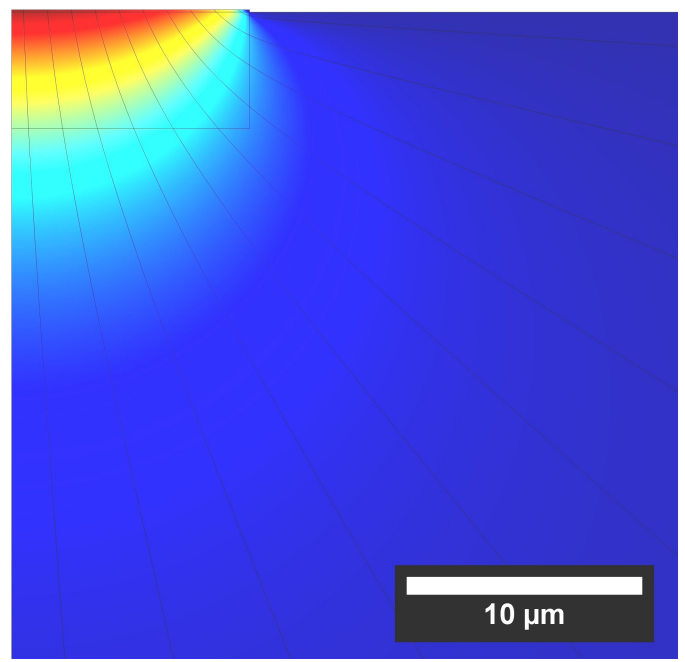

(b) „Platte“

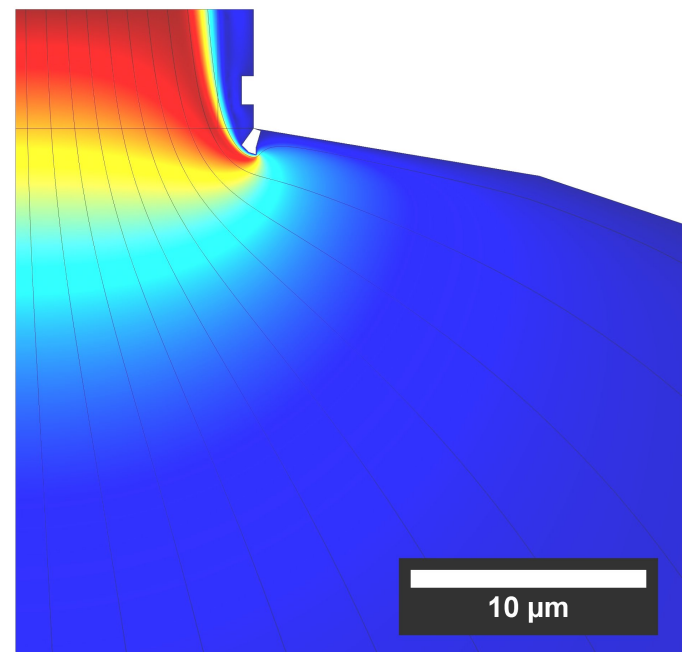

(d) ,Rohr*“

Abbildung D.2: Simulation (COMSOL) der absoluten Strömungsgeschwindigkeiten und Strömungslinien nahe der Düsenöffnung (jeweils am oberen Bildrand) für vier verschiedene Düsengeometrien. Rot entspricht einer vom Betrag hohen und blau einer vom Betrag niedrigen Geschwindigkeit.

Strömung (nämlich nur direkt an der Öffnung selbst) einen nur geringen Druckanstieg zur Folge hat. Im Falle der „Rohr“-Strömungen (Abb. D.2c und D.2d) ist ein deutlicher Druckanstieg zu sehen. Dieser ist zum einen von der zunehmenden Reibung aufgrund der vermehrten Nähe schneller Strömungen zur Düsenwand, als 
auch von der Strömung entlang der unebenen Düsenwand (was mit einer höheren Krümmung der Strömungslinien einhergeht) verursacht. Wie auch in Abb. D.2d ersichtlich, sind die Strömungslinien insbesondere an den hervorstehenden Unebenheiten der Düse gekrümmt, und der mit hoher Strömungsgeschwindigkeit durchflossene Bereich ist deutlich eingeschränkter.

Im Sinne eines möglichst geringen benötigten Druckes zur Erzeugung einer bestimmen Flussgeschwindigkeit ist also auf die Wahl eines strömungsoptimierten Düsendesigns zu achten [47. 


\section{Anhang $\mathrm{E}$}

\section{Schaltpläne der Hochspannungs- elektronik zur Tropfenaufladung}

Nachfolgend sind in Abb. E.1 und E.2, mit Genehmigung der Elektronischen Werkstatt des Dritten Physikalischen Instituts, die Schaltpläne der in Kap. 2 verwendeten Hochspannungselektronik zur Aufladung und Ablenkung einzelner Mikrotropfen abgebildet. In Abb. E.3 sind die Messungen der positiven und negativen Spannungspulse zur Tropfenaufladung mit unterschiedlichen Amplituden und Dauern gegeben. 


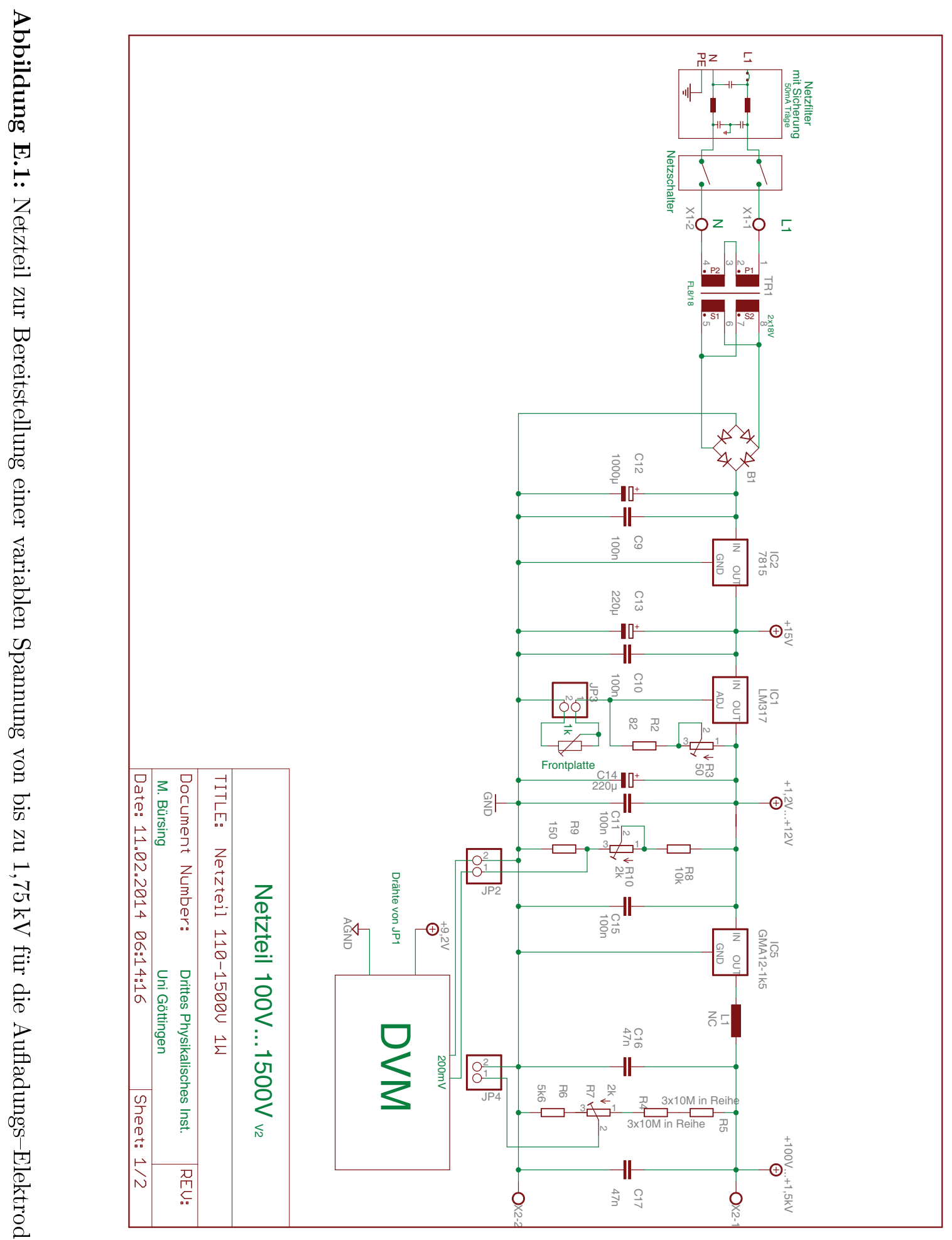




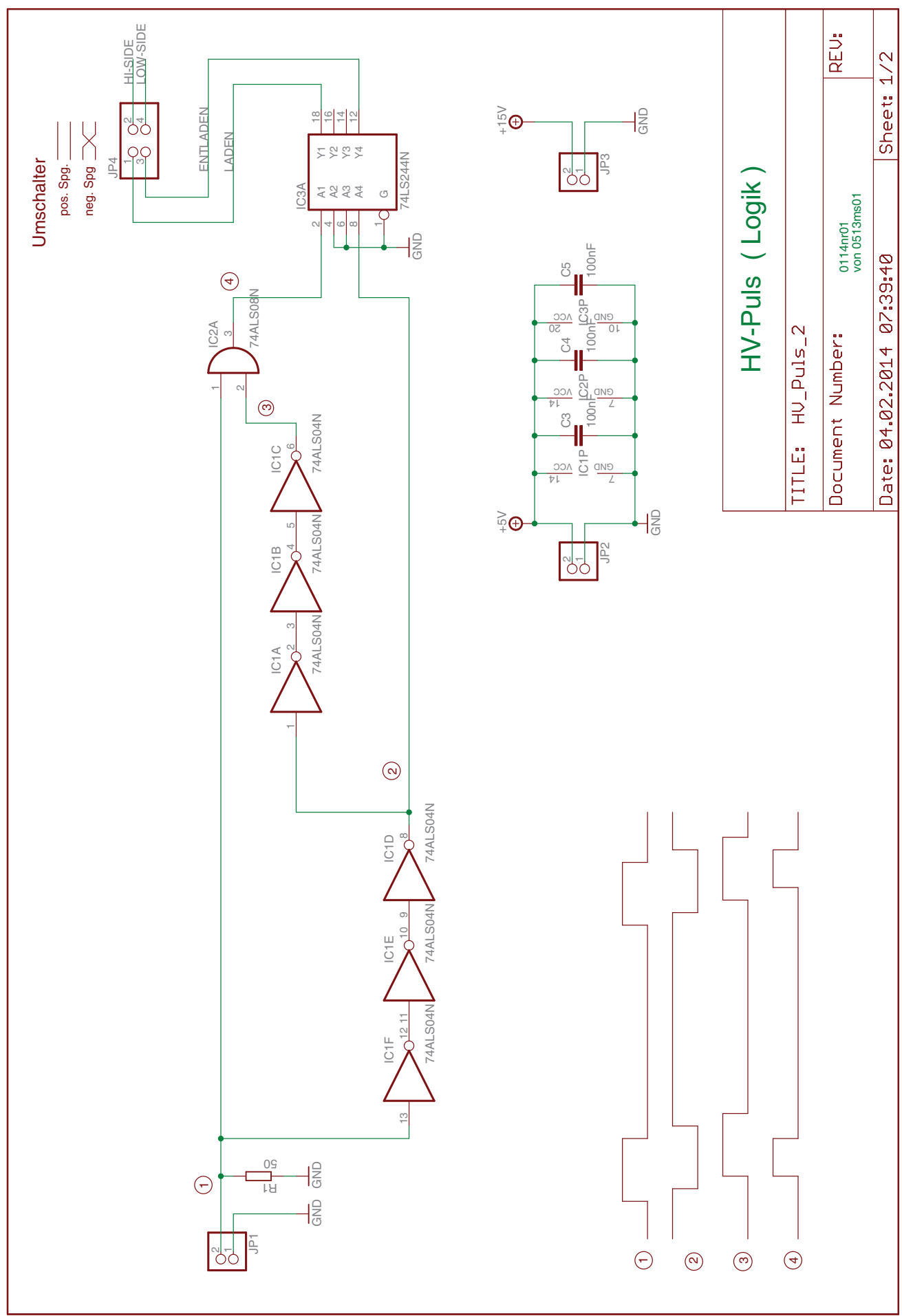

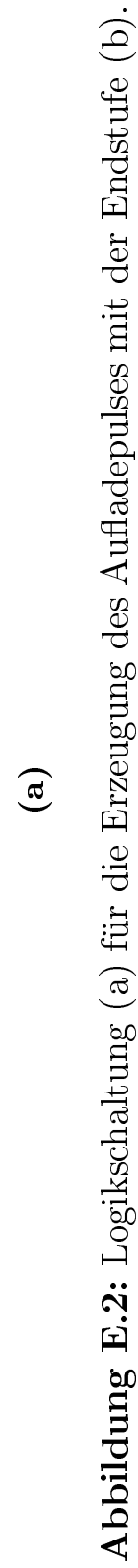




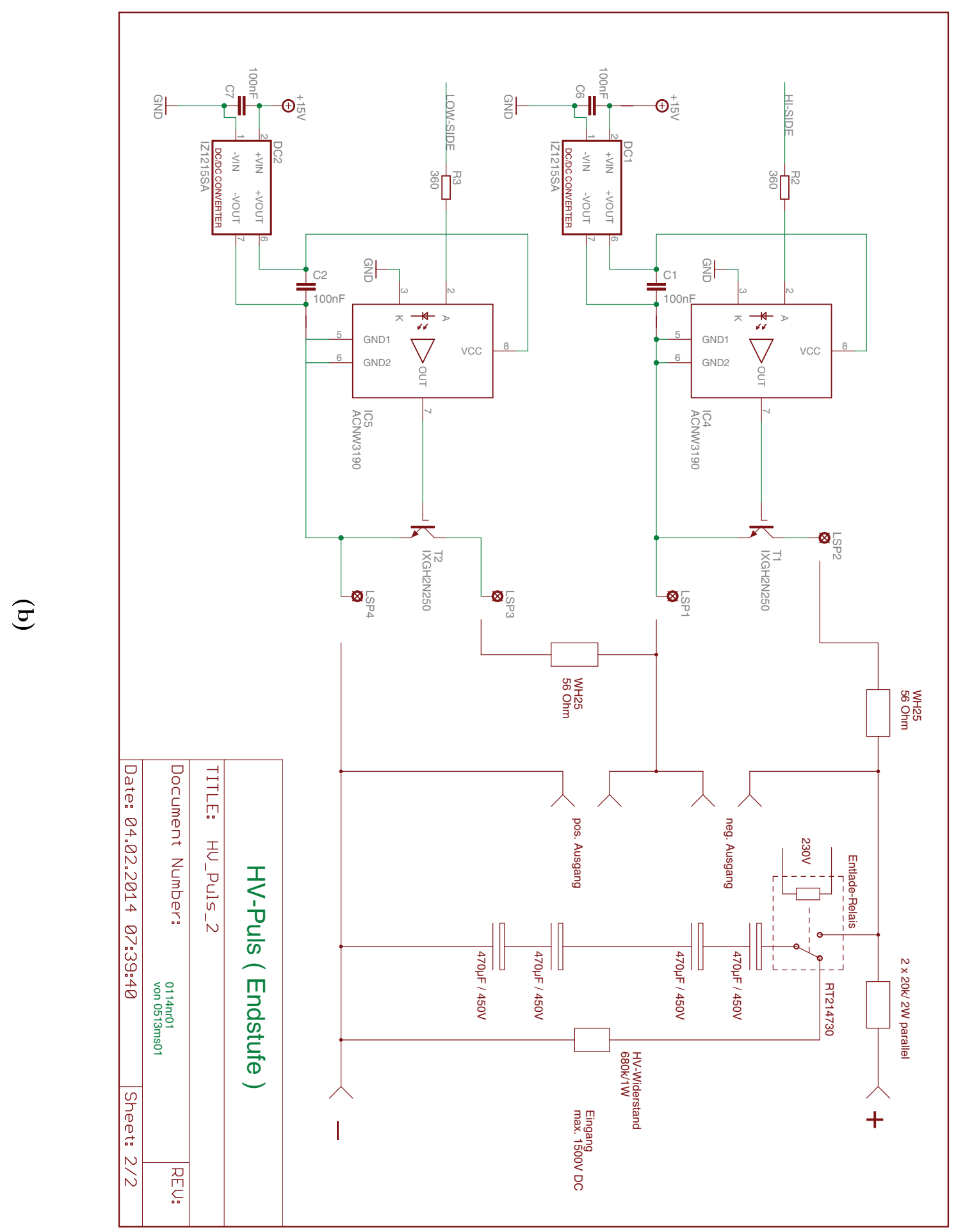




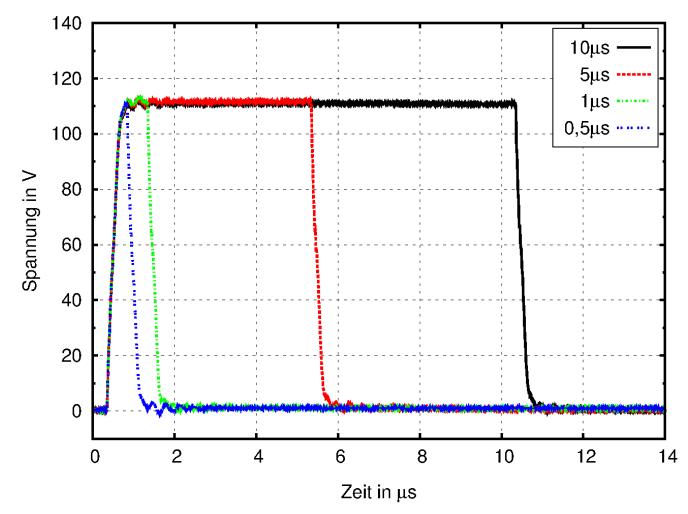

(a)

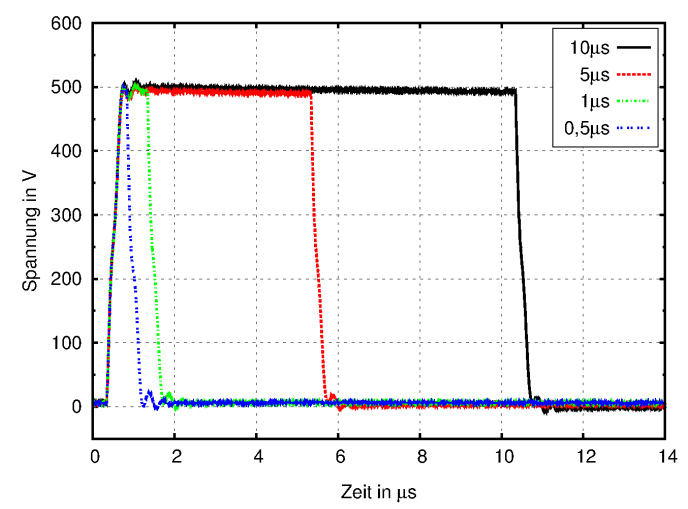

(c)

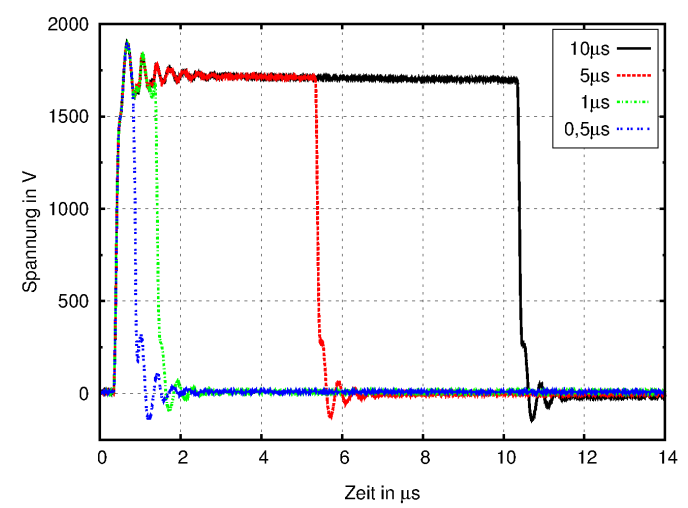

(e)

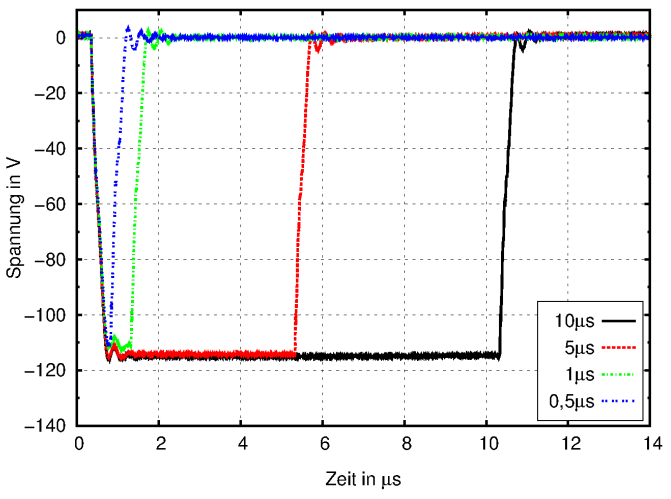

(b)

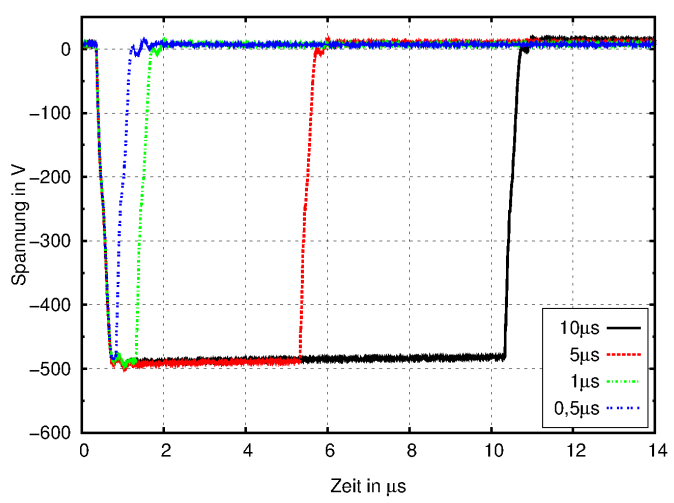

(d)

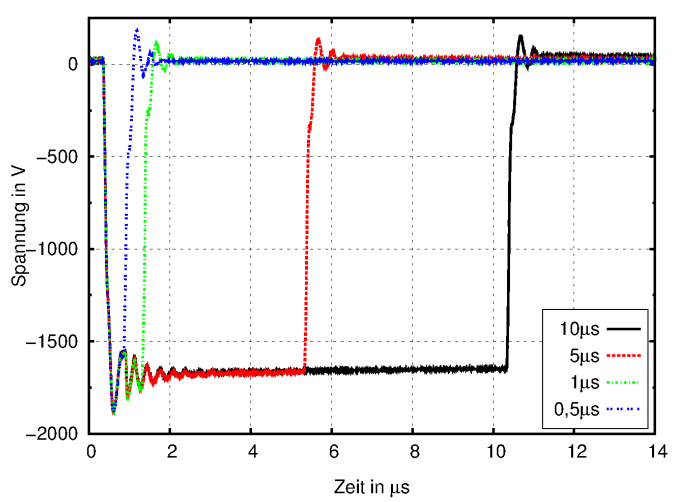

(f)

Abbildung E.3: Messung der erzeugbaren rechteckförmigen Hochspannungspulse für die Tropfenaufladung. Es sind sowohl (a)-(c) positive als auch (d)-(f) negative Pulse mit einer Amplitude bis zu ca. $1,75 \mathrm{kV}$ bei variabler Pulslänge möglich. Die Anstiegs- und Abfallzeit beträgt jeweils etwa 250 ns. Mit zunehmender Spannungsamplitude zeigen sich vermehrt „Überschwinger“. 



\section{Anhang $\mathrm{F}$ Überblick der PIV-Messungen zum Tropfeneinschlag auf ein rotierendes Substrat}

In Abb. F.1 bis Abb. F.12 sind alle Ergebnisse der PIV-Messungen gegeben. Es wurden vier verschiedene Tropfendurchmesser $D$ (ca. $20 \mu \mathrm{m}, 30 \mu \mathrm{m}, 40 \mu \mathrm{m}$ und $60 \mu \mathrm{m}$ ), drei verschiedene Geschwindigkeiten $U_{0}$ (ca. $30 \mathrm{~m} \mathrm{~s}^{-1}, 60 \mathrm{~m} \mathrm{~s}^{-1}$ und $90 \mathrm{~m} \mathrm{~s}^{-1}$ ) und drei verschiedene Dicken des Flüssigkeitsfilms $h$ (ca. $0 \mu \mathrm{m}, 40 \mu \mathrm{m}$ und $80 \mu \mathrm{m})$ untersucht. Für jeden Fall ist: 1.) die interpolierte Verteilung der am Substrat gemessenen Geschwindigkeit (linkes Bild), 2.) eine Pfeildarstellung der Strömungsrichtung und -geschwindigkeit (mittleres Bild) und 3.) eine beispielhafte Aufnahme der Einschlagsfläche im Durchlicht (rechtes Bild) gegeben. 

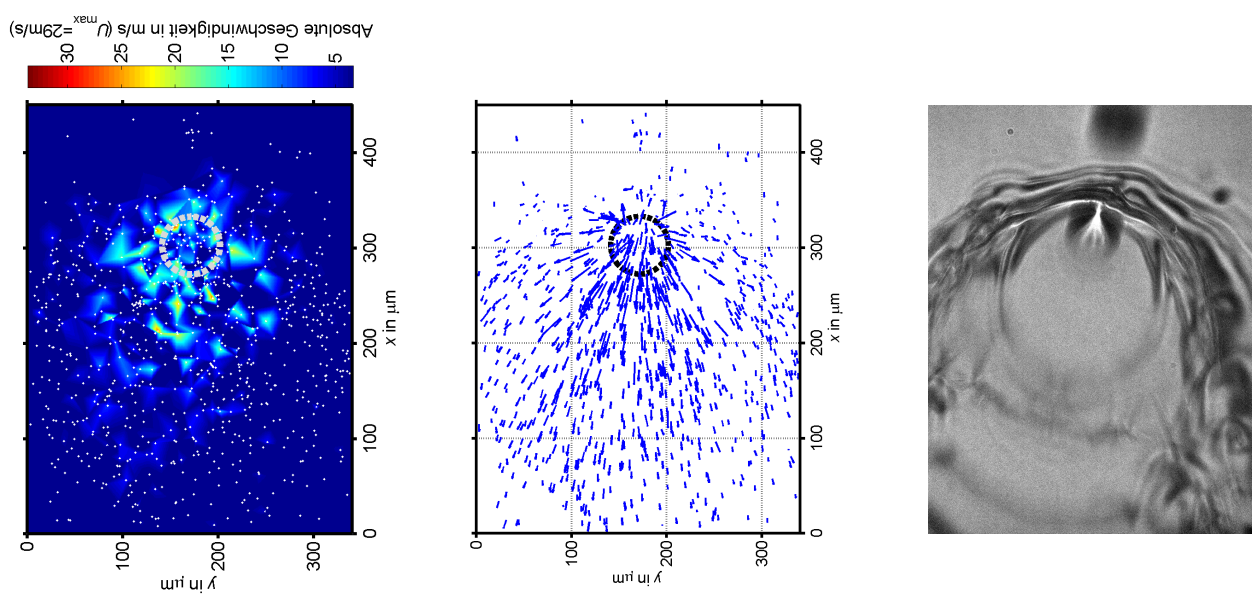

(a) $D=60 \mu \mathrm{m}, U_{0}=34 \mathrm{~m} \mathrm{~s}^{-1}$ und $h=0 \mu \mathrm{m}$
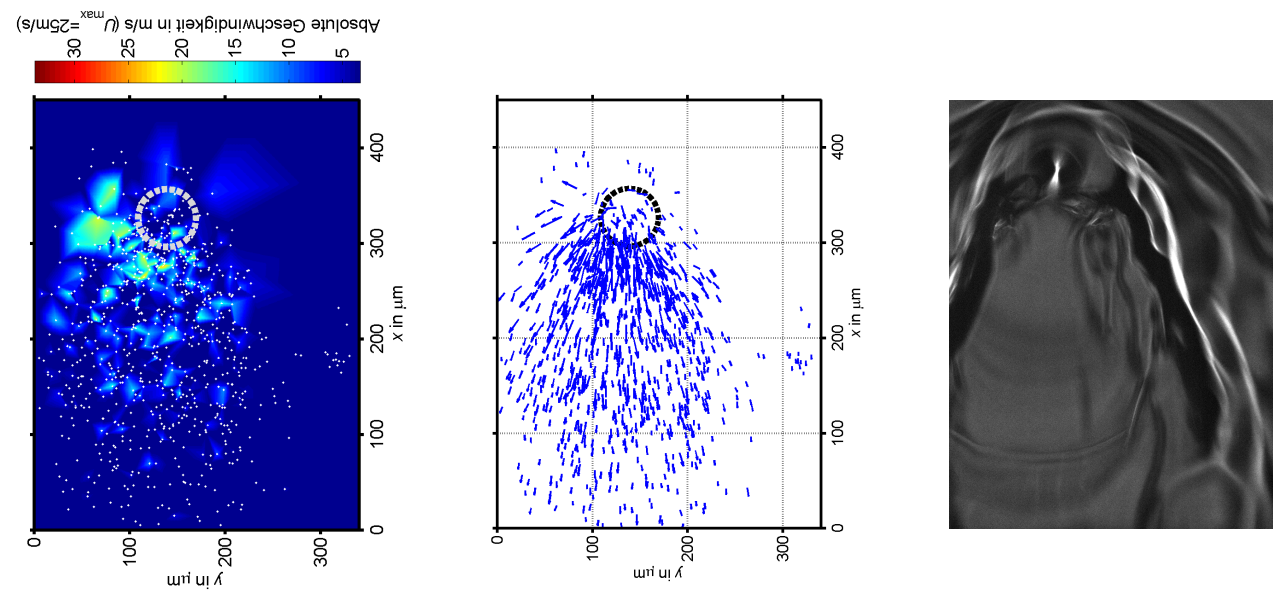

(b) $D=60 \mu \mathrm{m}, U_{0}=34 \mathrm{~m} \mathrm{~s}^{-1}$ und $h=40 \mu \mathrm{m}$
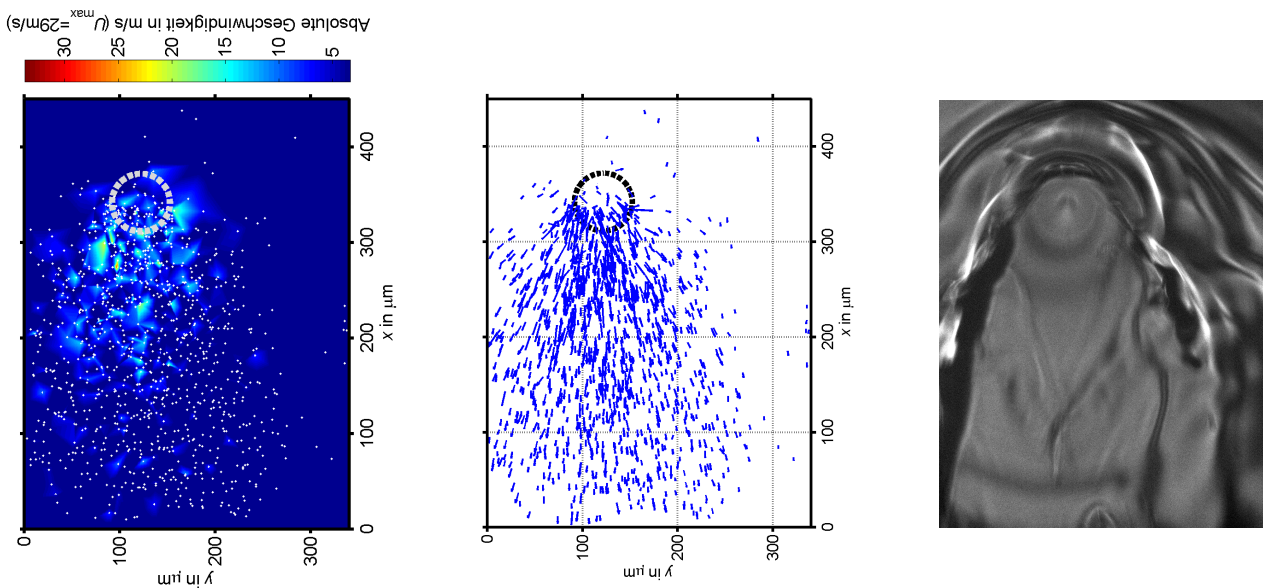

(c) $D=60 \mu \mathrm{m}, U_{0}=34 \mathrm{~m} \mathrm{~s}^{-1}$ und $h=80 \mu \mathrm{m}$

Abbildung F.1: Geschwindigkeit am Substrat für konstante Tropfengröße $D$ und Tropfengeschwindigkeit $U_{0}$ in Abhängigkeit von der Dicke des Flüssigkeitsfilms $h$. Geschwindigkeitsverteilung (linkes Bild), Pfeildarstellung der Strömungsrichtung und -geschwindigkeit (mittleres Bild) und Aufnahme im Durchlicht (rechtes Bild). Weiße Punkte im linken Bild entsprechen den Messpunkten/Pfeilen im mittleren Bild. Ein gestrichelter Kreis zeigt die Tropfengröße und Einschlagsposition. 

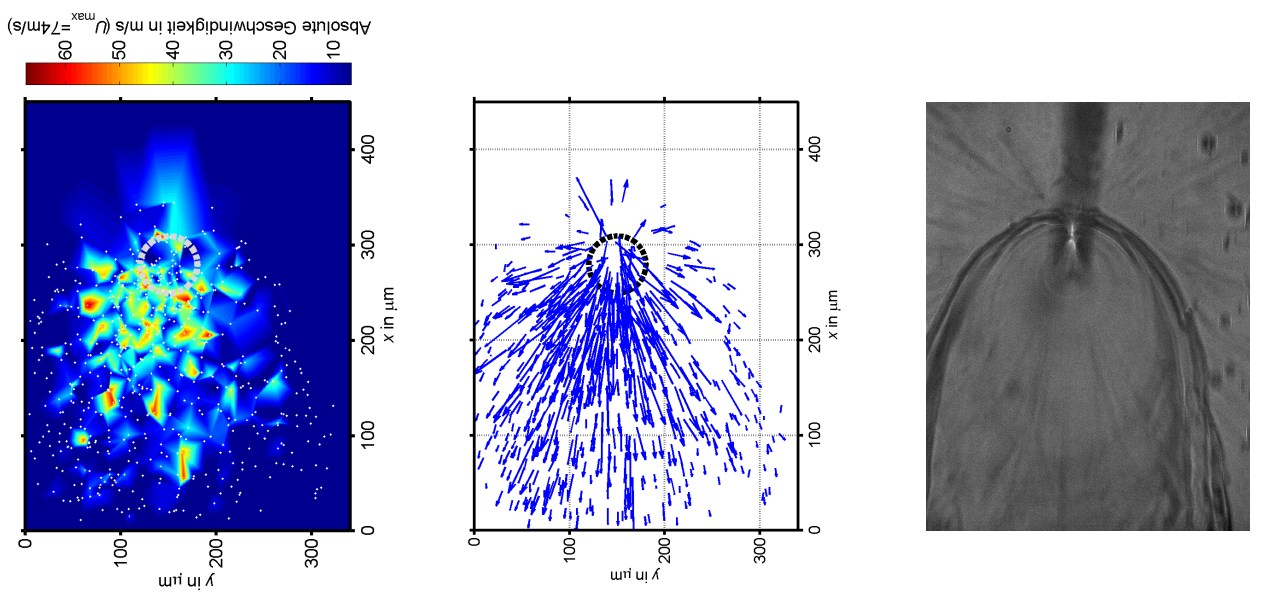

(a) $D=60 \mu \mathrm{m}, U_{0}=67 \mathrm{~m} \mathrm{~s}^{-1}$ und $h=0 \mu \mathrm{m}$
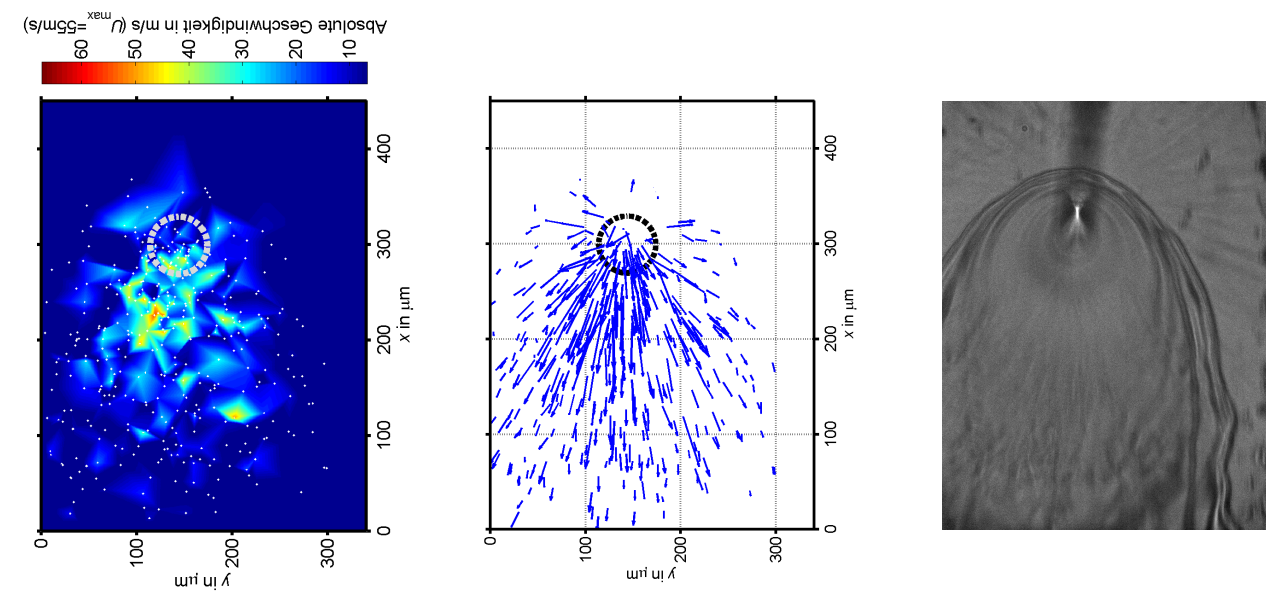

(b) $D=60 \mu \mathrm{m}, U_{0}=67 \mathrm{~m} \mathrm{~s}^{-1}$ und $h=40 \mu \mathrm{m}$
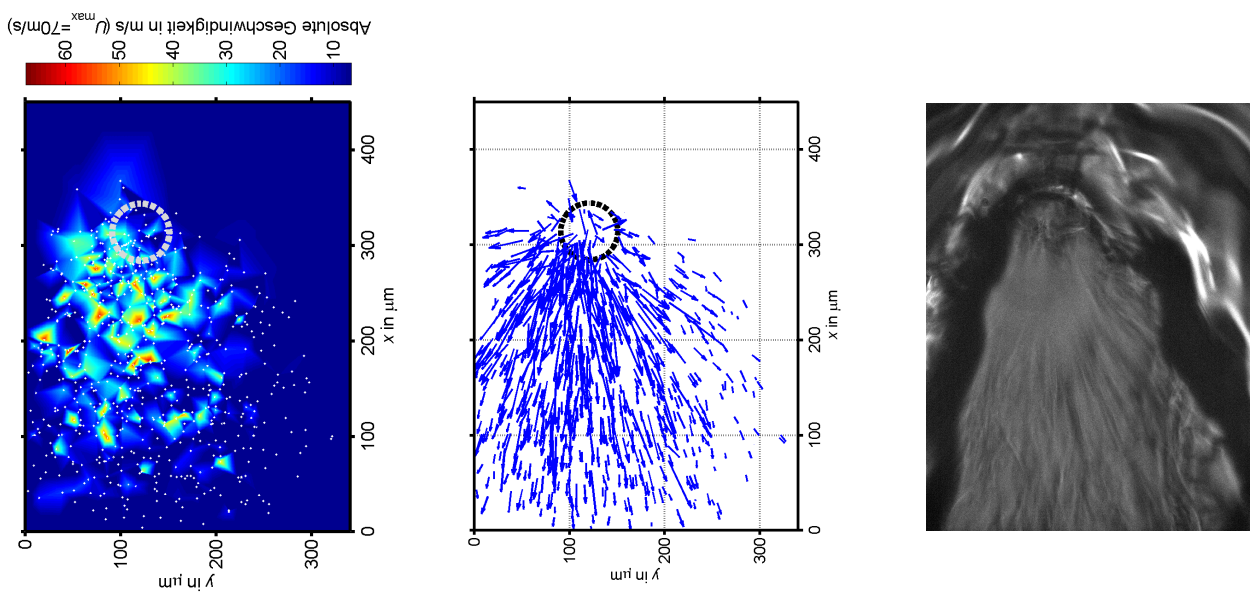

(c) $D=60 \mu \mathrm{m}, U_{0}=67 \mathrm{~m} \mathrm{~s}^{-1}$ und $h=80 \mu \mathrm{m}$

Abbildung F.2: Geschwindigkeit am Substrat für konstante Tropfengröße $D$ und Tropfengeschwindigkeit $U_{0}$ in Abhängigkeit von der Dicke des Flüssigkeitsfilms $h$. Geschwindigkeitsverteilung (linkes Bild), Pfeildarstellung der Strömungsrichtung und -geschwindigkeit (mittleres Bild) und Aufnahme im Durchlicht (rechtes Bild). Weiße Punkte im linken Bild entsprechen den Messpunkten/Pfeilen im mittleren Bild. Ein gestrichelter Kreis zeigt die Tropfengröße und Einschlagsposition. 

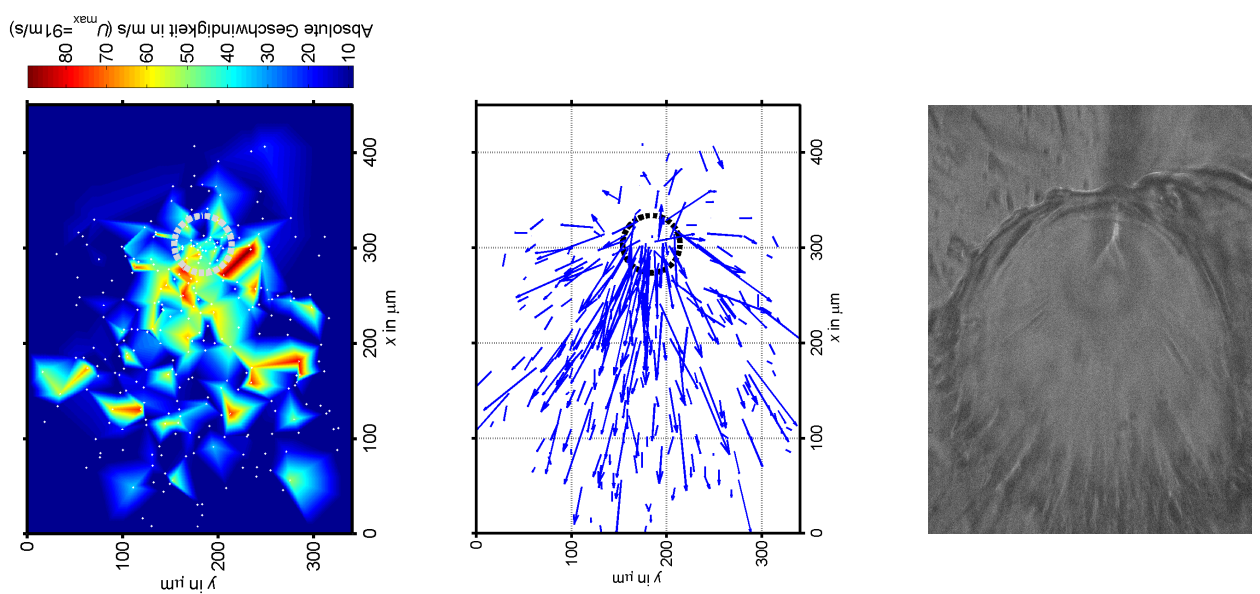

(a) $D=60 \mu \mathrm{m}, U_{0}=90 \mathrm{~m} \mathrm{~s}^{-1}$ und $h=0 \mu \mathrm{m}$
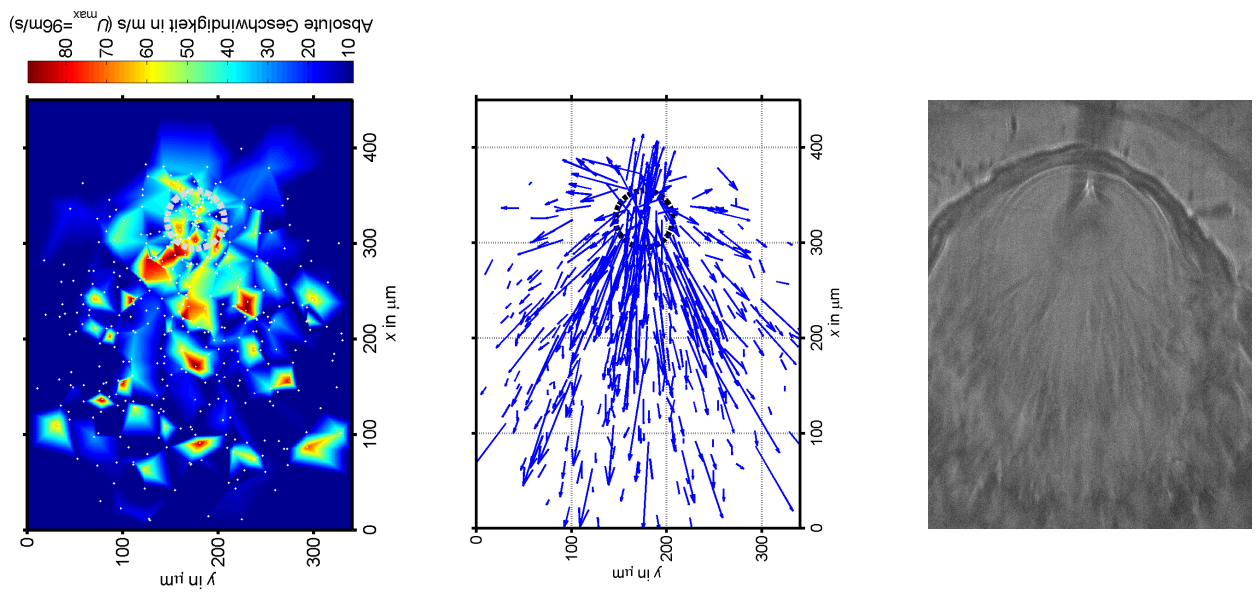

(b) $D=60 \mu \mathrm{m}, U_{0}=90 \mathrm{~m} \mathrm{~s}^{-1}$ und $h=40 \mu \mathrm{m}$
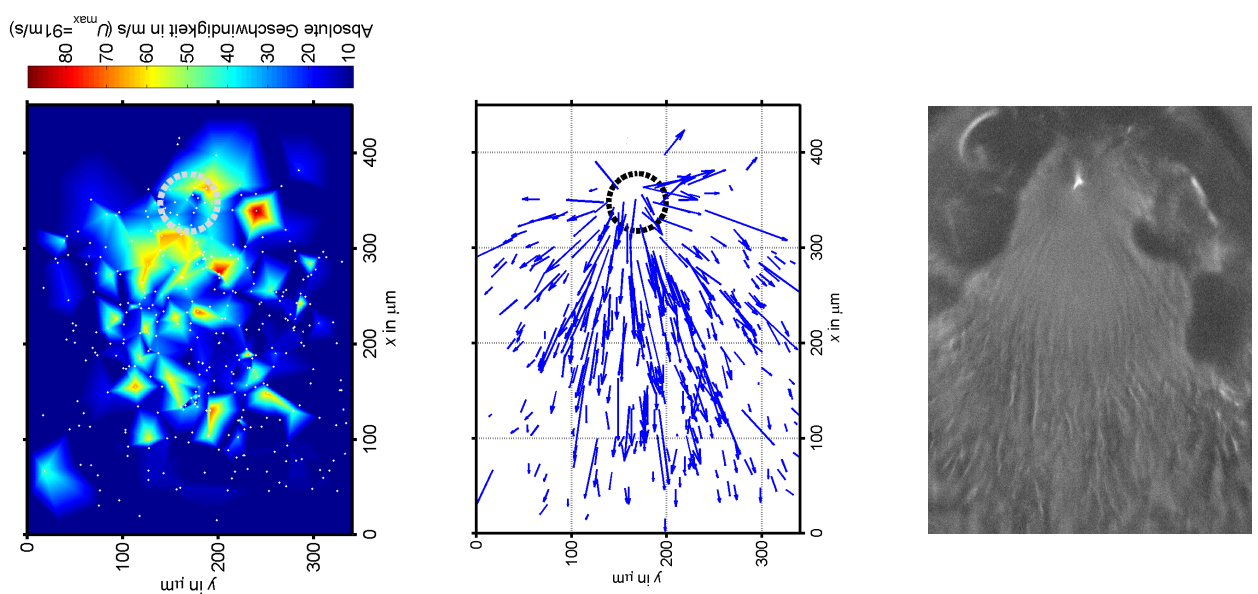

(c) $D=60 \mu \mathrm{m}, U_{0}=90 \mathrm{~m} \mathrm{~s}^{-1}$ und $h=80 \mu \mathrm{m}$

Abbildung F.3: Geschwindigkeit am Substrat für konstante Tropfengröße $D$ und Tropfengeschwindigkeit $U_{0}$ in Abhängigkeit von der Dicke des Flüssigkeitsfilms $h$. Geschwindigkeitsverteilung (linkes Bild), Pfeildarstellung der Strömungsrichtung und -geschwindigkeit (mittleres Bild) und Aufnahme im Durchlicht (rechtes Bild). Weiße Punkte im linken Bild entsprechen den Messpunkten/Pfeilen im mittleren Bild. Ein gestrichelter Kreis zeigt die Tropfengröße und Einschlagsposition. 

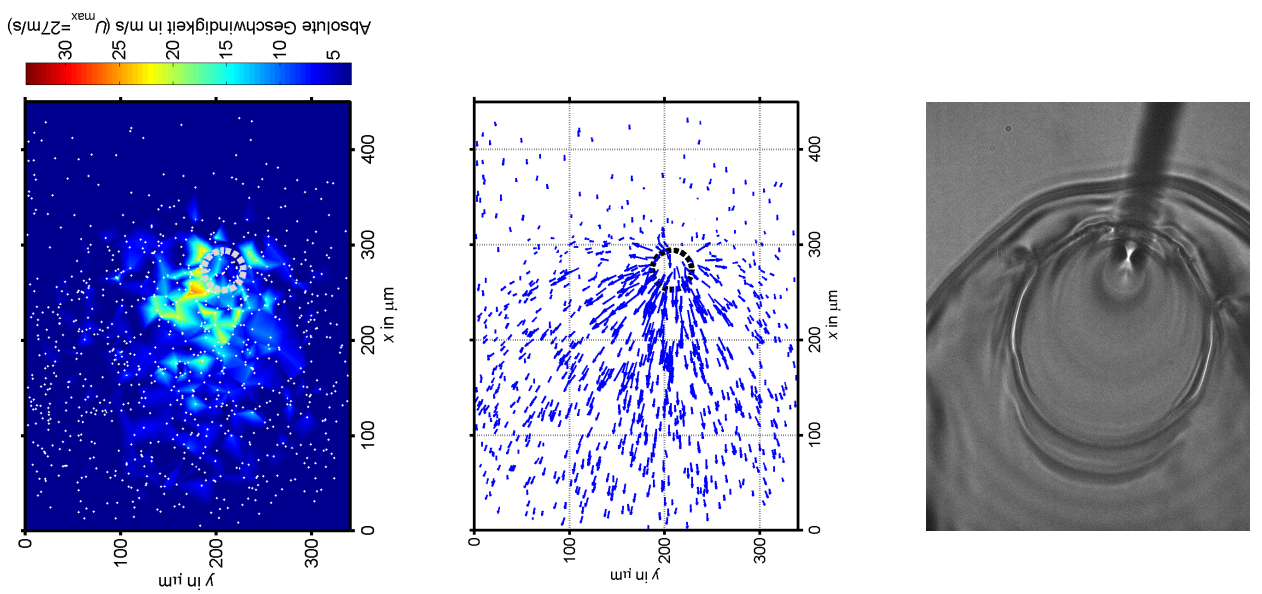

(a) $D=41 \mu \mathrm{m}, U_{0}=34 \mathrm{~m} \mathrm{~s}^{-1}$ und $h=0 \mu \mathrm{m}$
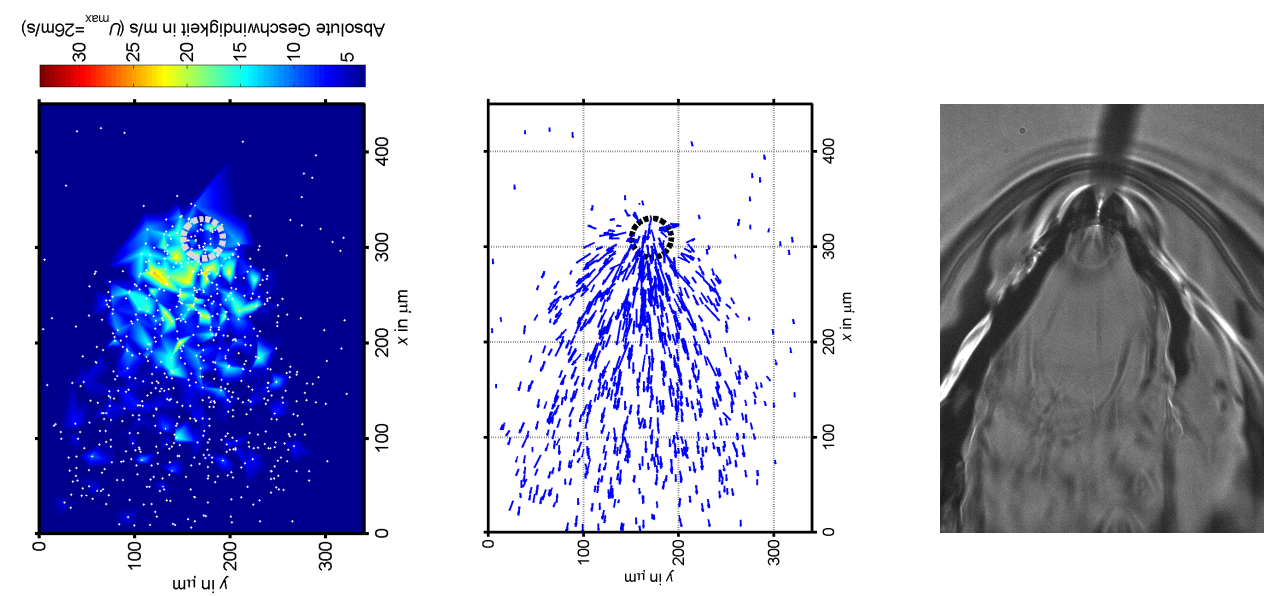

(b) $D=41 \mu \mathrm{m}, U_{0}=34 \mathrm{~m} \mathrm{~s}^{-1}$ und $h=40 \mu \mathrm{m}$
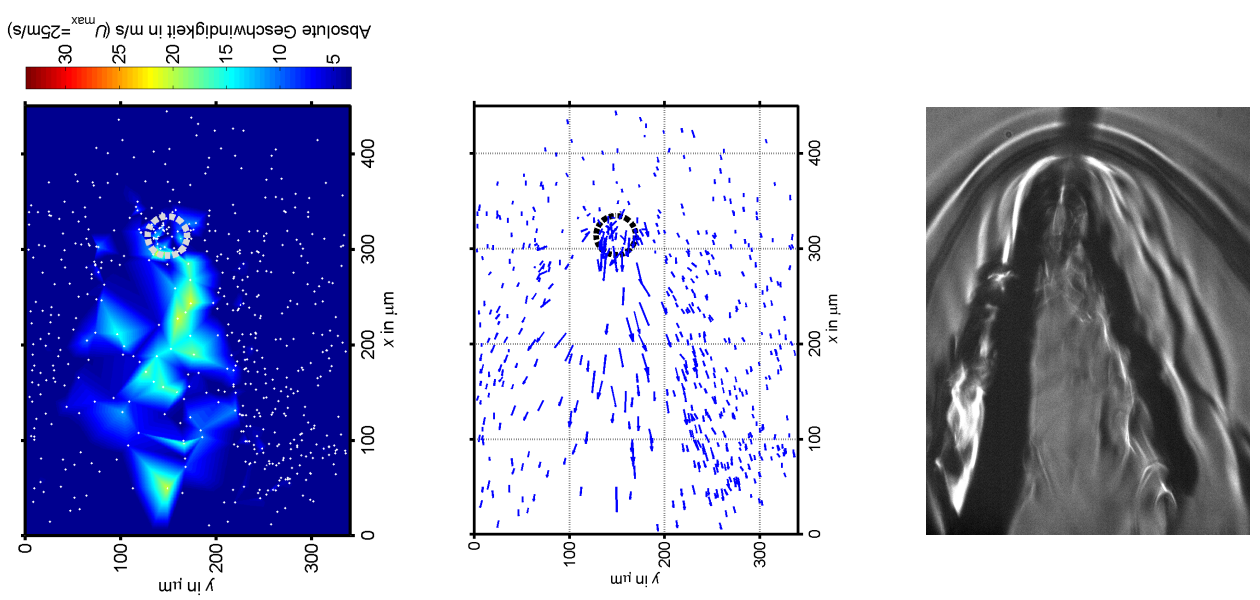

(c) $D=41 \mu \mathrm{m}, U_{0}=34 \mathrm{~m} \mathrm{~s}^{-1}$ und $h=80 \mu \mathrm{m}$

Abbildung F.4: Geschwindigkeit am Substrat für konstante Tropfengröße $D$ und Tropfengeschwindigkeit $U_{0}$ in Abhängigkeit von der Dicke des Flüssigkeitsfilms $h$. Geschwindigkeitsverteilung (linkes Bild), Pfeildarstellung der Strömungsrichtung und -geschwindigkeit (mittleres Bild) und Aufnahme im Durchlicht (rechtes Bild). Weiße Punkte im linken Bild entsprechen den Messpunkten/Pfeilen im mittleren Bild. Ein gestrichelter Kreis zeigt die Tropfengröße und Einschlagsposition. 

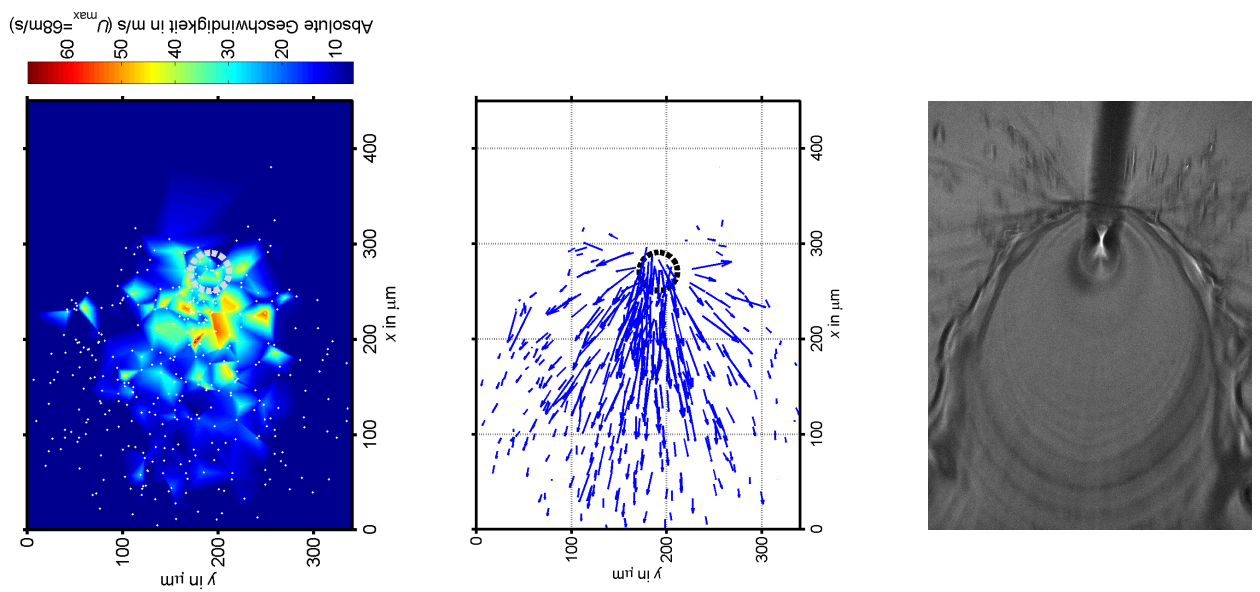

(a) $D=40 \mu \mathrm{m}, U_{0}=67 \mathrm{~m} \mathrm{~s}^{-1}$ und $h=0 \mu \mathrm{m}$
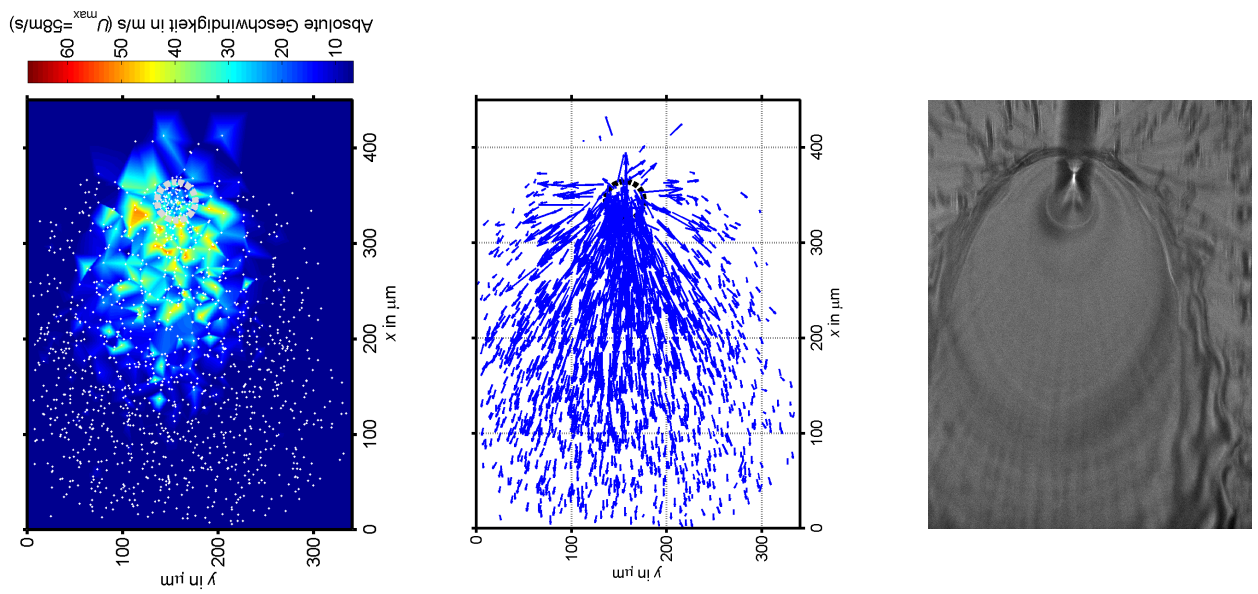

(b) $D=40 \mu \mathrm{m}, U_{0}=67 \mathrm{~m} \mathrm{~s}^{-1}$ und $h=40 \mu \mathrm{m}$
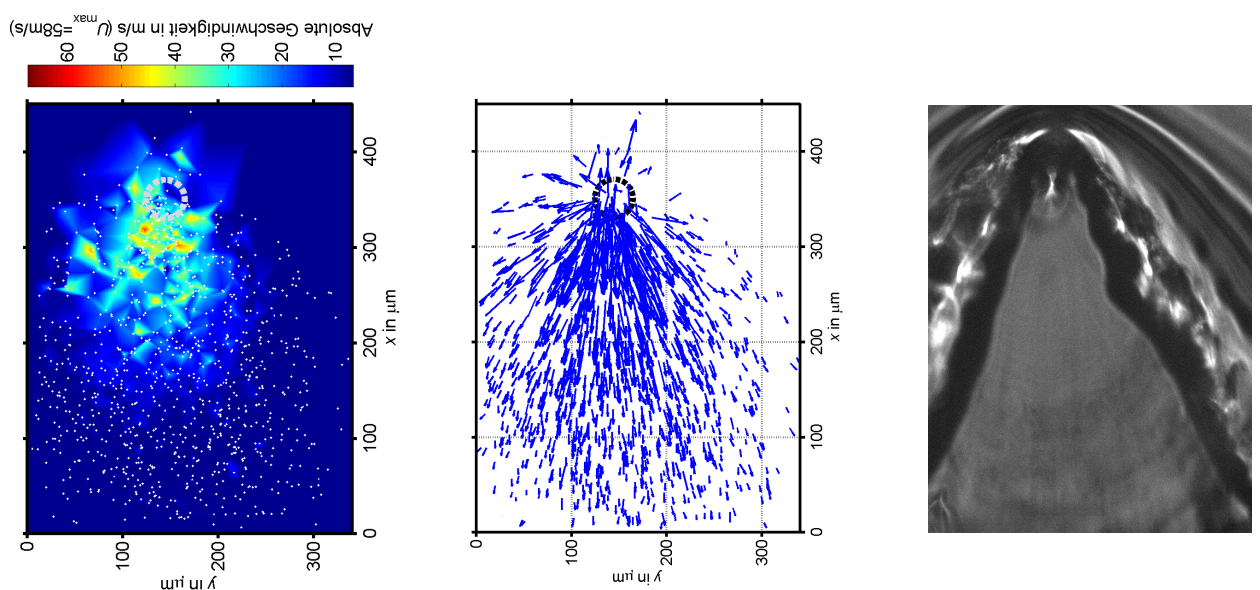

(c) $D=40 \mu \mathrm{m}, U_{0}=67 \mathrm{~m} \mathrm{~s}^{-1}$ und $h=80 \mu \mathrm{m}$

Abbildung F.5: Geschwindigkeit am Substrat für konstante Tropfengröße $D$ und Tropfengeschwindigkeit $U_{0}$ in Abhängigkeit von der Dicke des Flüssigkeitsfilms $h$. Geschwindigkeitsverteilung (linkes Bild), Pfeildarstellung der Strömungsrichtung und -geschwindigkeit (mittleres Bild) und Aufnahme im Durchlicht (rechtes Bild). Weiße Punkte im linken Bild entsprechen den Messpunkten/Pfeilen im mittleren Bild. Ein gestrichelter Kreis zeigt die Tropfengröße und Einschlagsposition. 

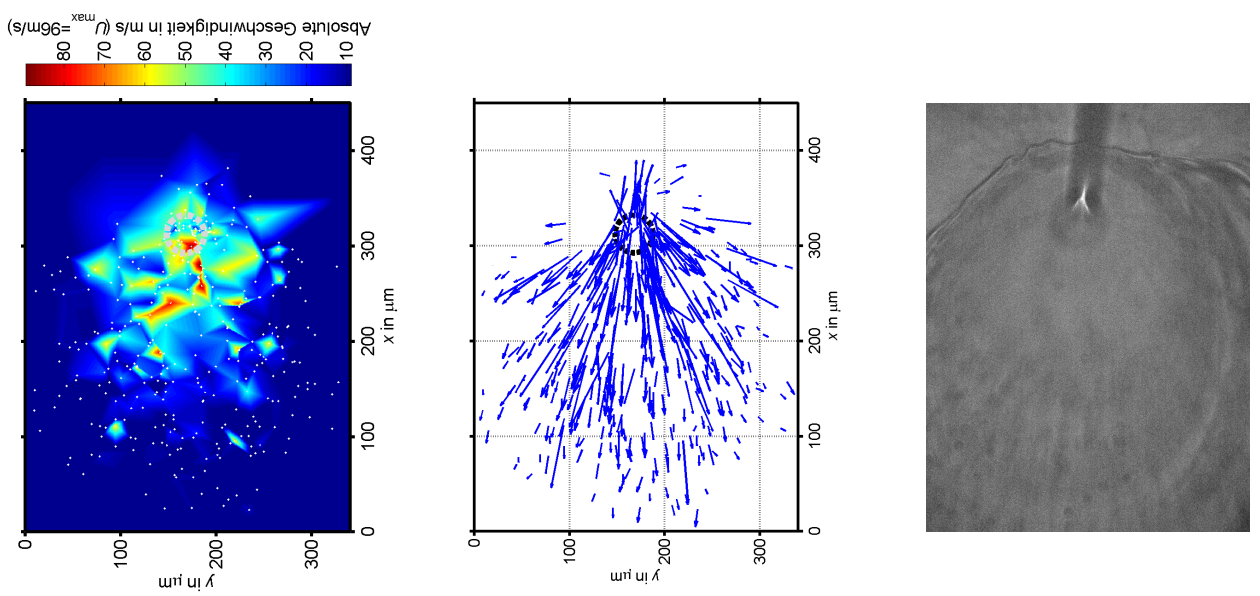

(a) $D=40 \mu \mathrm{m}, U_{0}=90 \mathrm{~m} \mathrm{~s}^{-1}$ und $h=0 \mu \mathrm{m}$
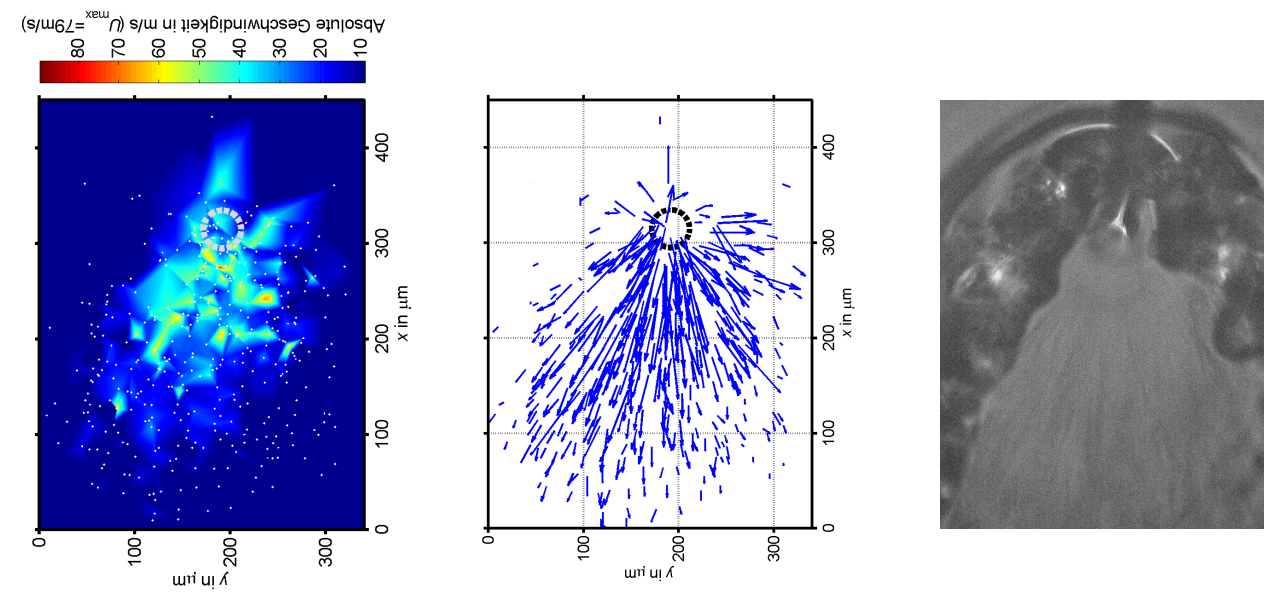

(b) $D=40 \mu \mathrm{m}, U_{0}=90 \mathrm{~m} \mathrm{~s}^{-1}$ und $h=40 \mu \mathrm{m}$
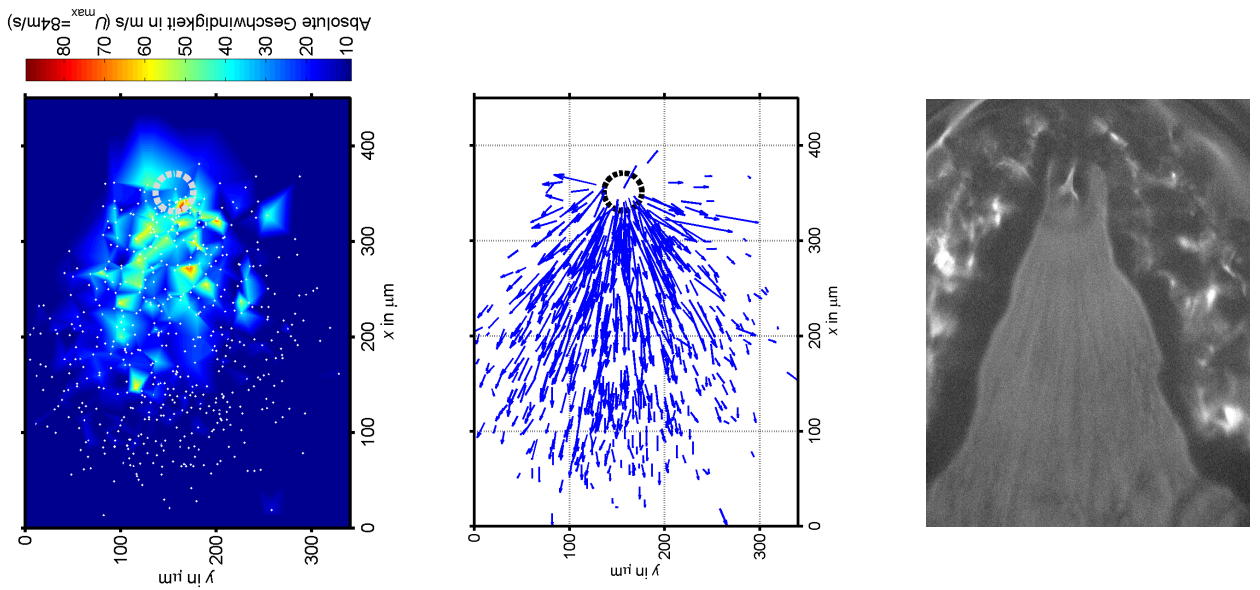

(c) $D=40 \mu \mathrm{m}, U_{0}=90 \mathrm{~m} \mathrm{~s}^{-1}$ und $h=80 \mu \mathrm{m}$

Abbildung F.6: Geschwindigkeit am Substrat für konstante Tropfengröße $D$ und Tropfengeschwindigkeit $U_{0}$ in Abhängigkeit von der Dicke des Flüssigkeitsfilms $h$. Geschwindigkeitsverteilung (linkes Bild), Pfeildarstellung der Strömungsrichtung und -geschwindigkeit (mittleres Bild) und Aufnahme im Durchlicht (rechtes Bild). Weiße Punkte im linken Bild entsprechen den Messpunkten/Pfeilen im mittleren Bild. Ein gestrichelter Kreis zeigt die Tropfengröße und Einschlagsposition. 

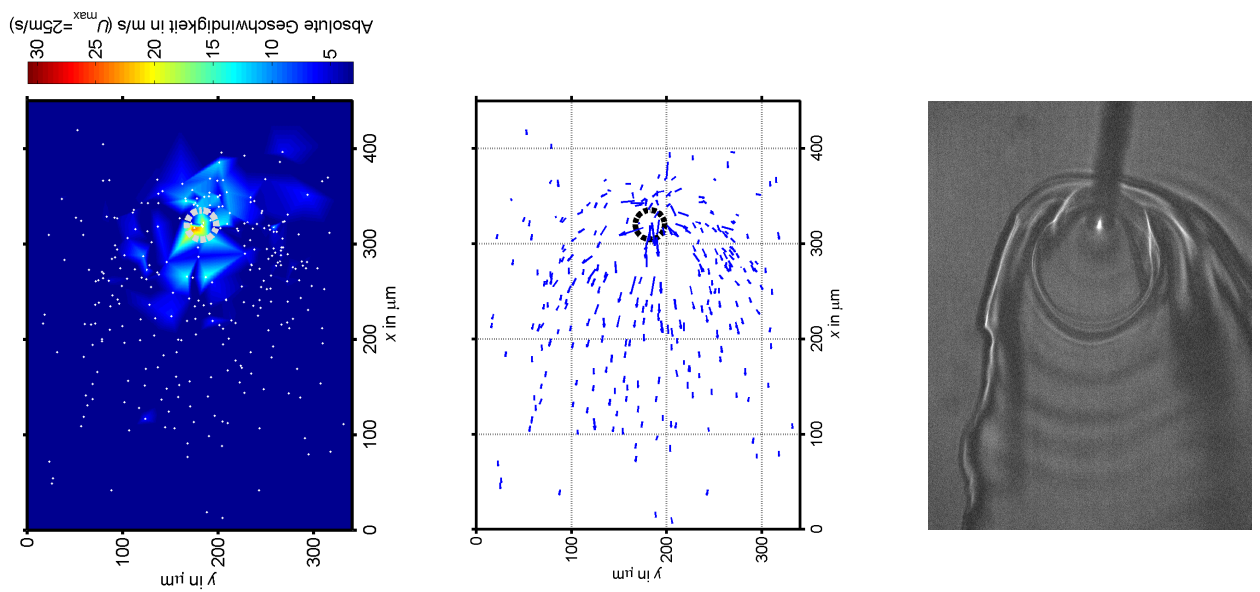

(a) $D=30 \mu \mathrm{m}, U_{0}=31 \mathrm{~m} \mathrm{~s}^{-1}$ und $h=0 \mu \mathrm{m}$
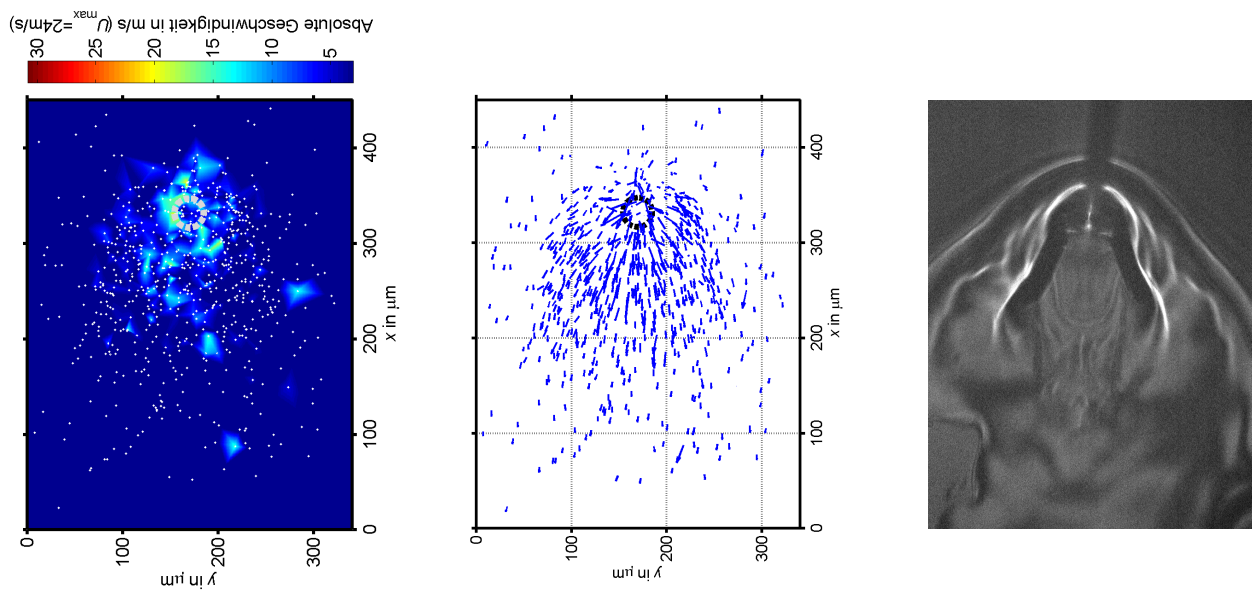

(b) $D=30 \mu \mathrm{m}, U_{0}=31 \mathrm{~m} \mathrm{~s}^{-1}$ und $h=40 \mu \mathrm{m}$
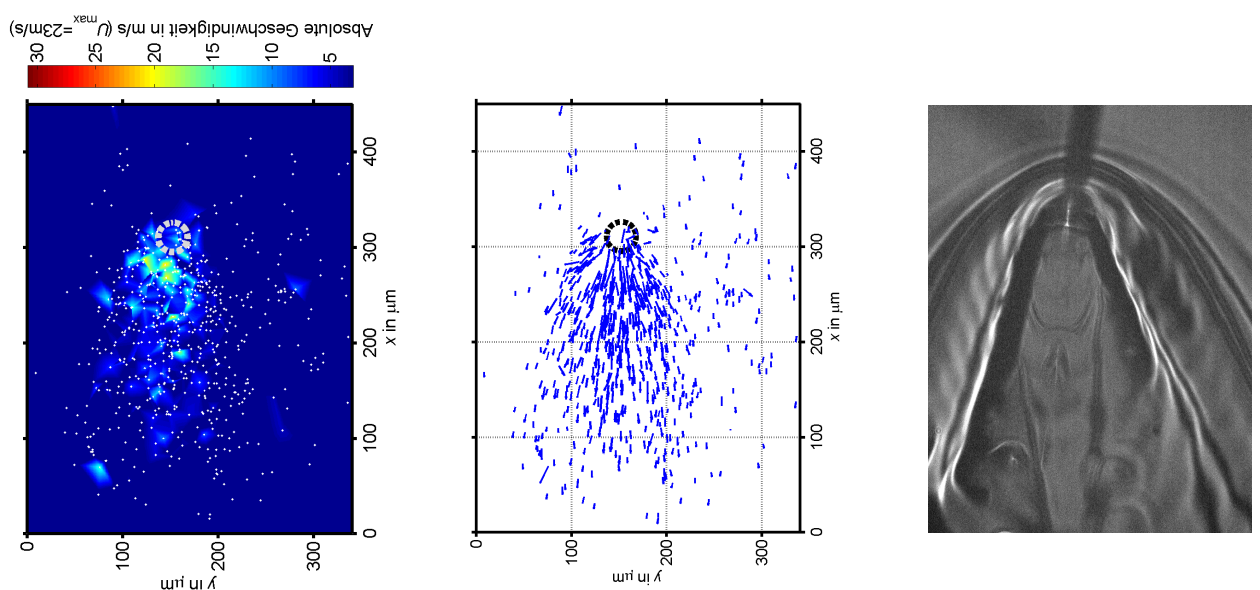

(c) $D=30 \mu \mathrm{m}, U_{0}=31 \mathrm{~m} \mathrm{~s}^{-1}$ und $h=80 \mu \mathrm{m}$

Abbildung F.7: Geschwindigkeit am Substrat für konstante Tropfengröße $D$ und Tropfengeschwindigkeit $U_{0}$ in Abhängigkeit von der Dicke des Flüssigkeitsfilms $h$. Geschwindigkeitsverteilung (linkes Bild), Pfeildarstellung der Strömungsrichtung und -geschwindigkeit (mittleres Bild) und Aufnahme im Durchlicht (rechtes Bild). Weiße Punkte im linken Bild entsprechen den Messpunkten/Pfeilen im mittleren Bild. Ein gestrichelter Kreis zeigt die Tropfengröße und Einschlagsposition. 

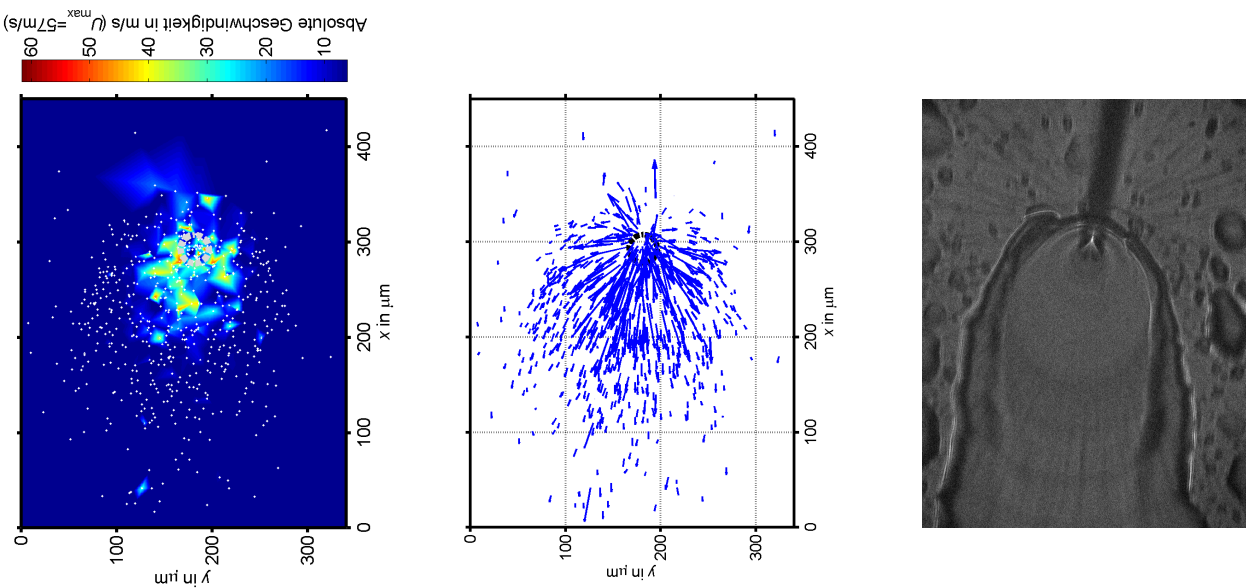

(a) $D=29 \mu \mathrm{m}, U_{0}=62 \mathrm{~m} \mathrm{~s}^{-1}$ und $h=0 \mu \mathrm{m}$
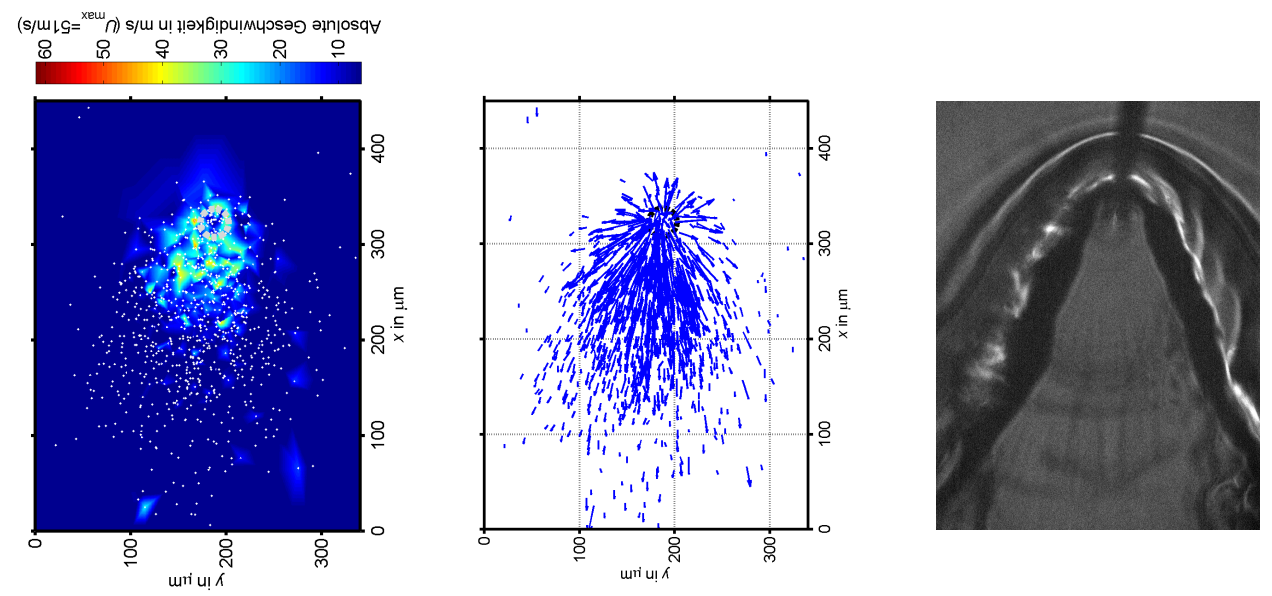

(b) $D=29 \mu \mathrm{m}, U_{0}=62 \mathrm{~m} \mathrm{~s}^{-1}$ und $h=40 \mu \mathrm{m}$
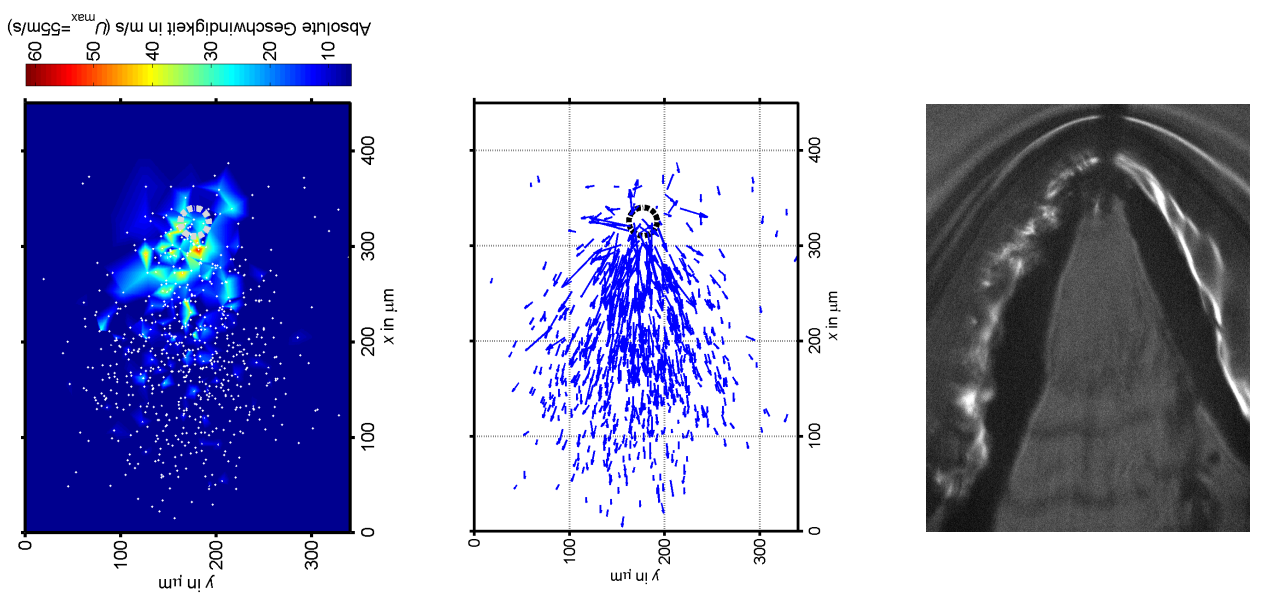

(c) $D=29 \mu \mathrm{m}, U_{0}=62 \mathrm{~m} \mathrm{~s}^{-1}$ und $h=80 \mu \mathrm{m}$

Abbildung F.8: Geschwindigkeit am Substrat für konstante Tropfengröße $D$ und Tropfengeschwindigkeit $U_{0}$ in Abhängigkeit von der Dicke des Flüssigkeitsfilms $h$. Geschwindigkeitsverteilung (linkes Bild), Pfeildarstellung der Strömungsrichtung und -geschwindigkeit (mittleres Bild) und Aufnahme im Durchlicht (rechtes Bild). Weiße Punkte im linken Bild entsprechen den Messpunkten/Pfeilen im mittleren Bild. Ein gestrichelter Kreis zeigt die Tropfengröße und Einschlagsposition. 

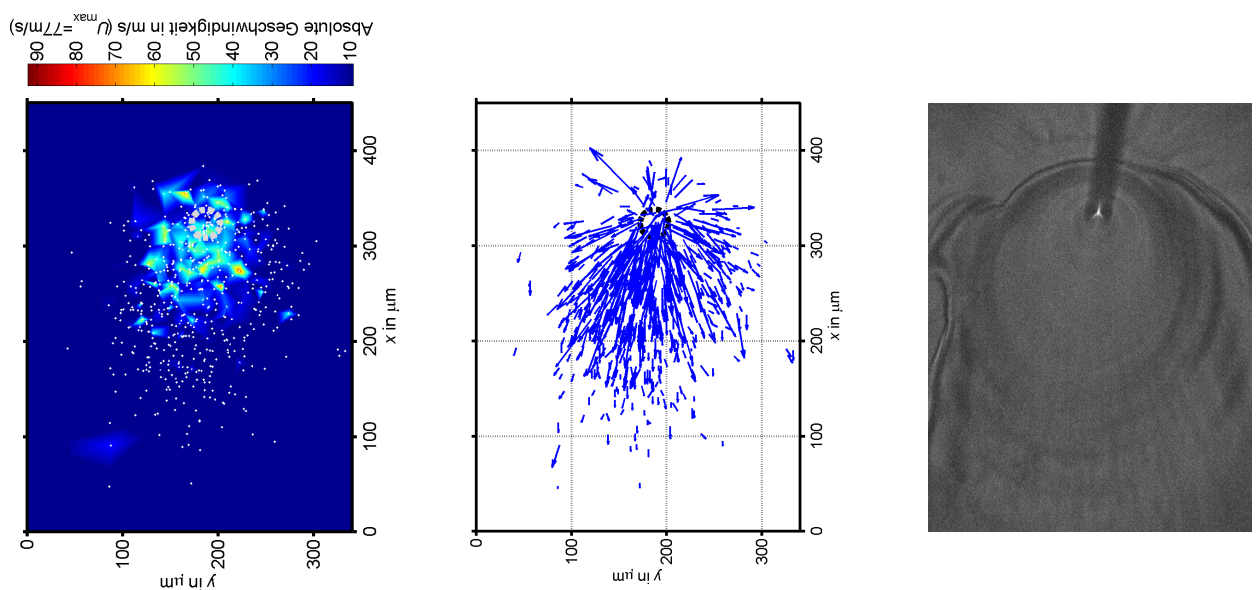

(a) $D=29 \mu \mathrm{m}, U_{0}=92 \mathrm{~m} \mathrm{~s}^{-1}$ und $h=0 \mu \mathrm{m}$
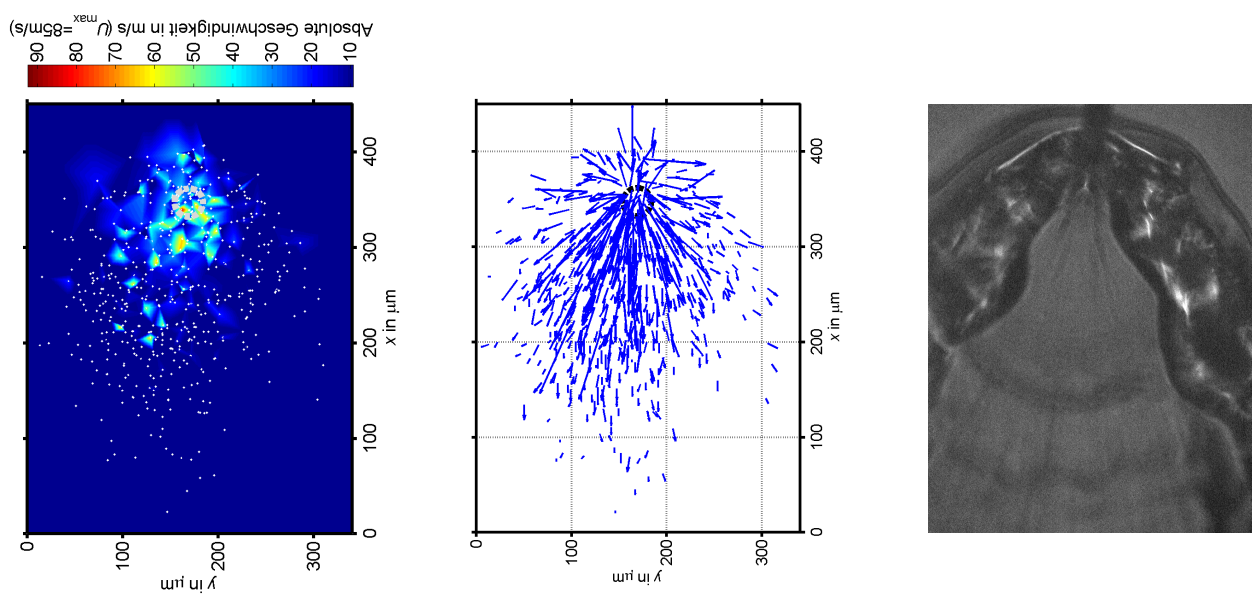

(b) $D=29 \mu \mathrm{m}, U_{0}=92 \mathrm{~m} \mathrm{~s}^{-1}$ und $h=40 \mu \mathrm{m}$
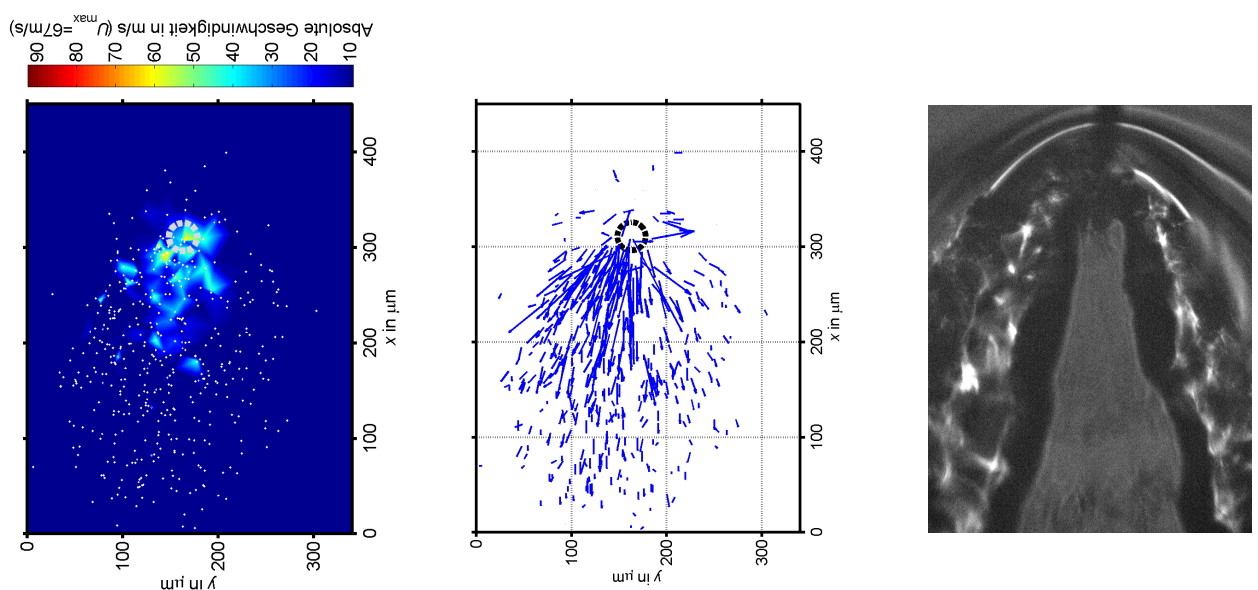

(c) $D=29 \mu \mathrm{m}, U_{0}=92 \mathrm{~m} \mathrm{~s}^{-1}$ und $h=80 \mu \mathrm{m}$

Abbildung F.9: Geschwindigkeit am Substrat für konstante Tropfengröße $D$ und Tropfengeschwindigkeit $U_{0}$ in Abhängigkeit von der Dicke des Flüssigkeitsfilms $h$. Geschwindigkeitsverteilung (linkes Bild), Pfeildarstellung der Strömungsrichtung und -geschwindigkeit (mittleres Bild) und Aufnahme im Durchlicht (rechtes Bild). Weiße Punkte im linken Bild entsprechen den Messpunkten/Pfeilen im mittleren Bild. Ein gestrichelter Kreis zeigt die Tropfengröße und Einschlagsposition. 

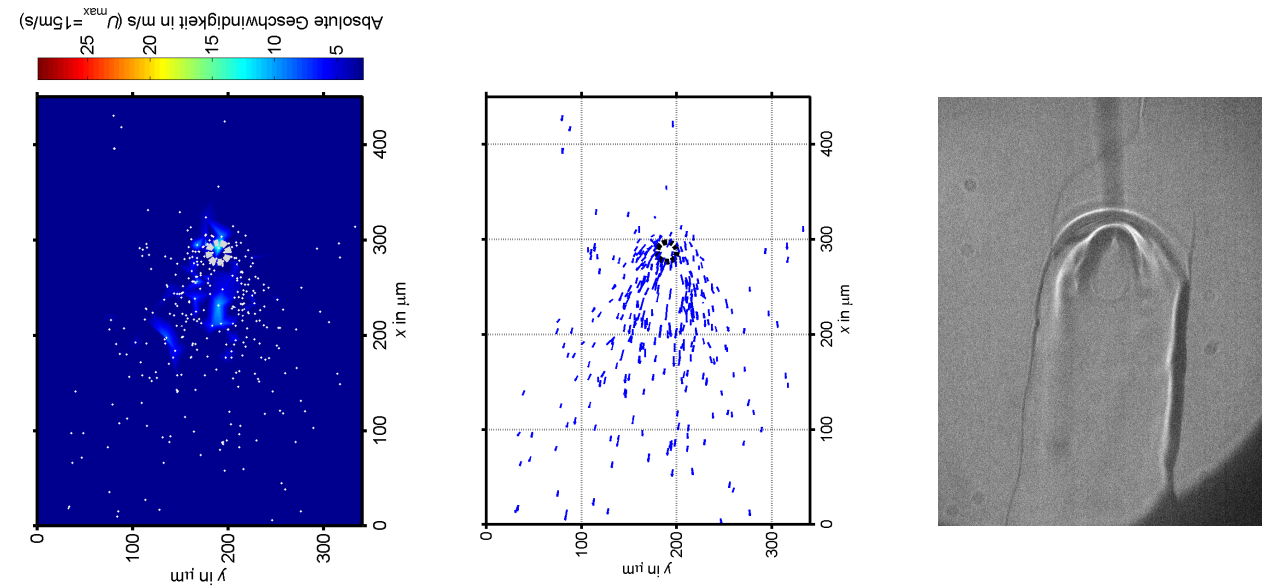

(a) $D=20 \mu \mathrm{m}, U_{0}=29 \mathrm{~m} \mathrm{~s}^{-1}$ und $h=0 \mu \mathrm{m}$
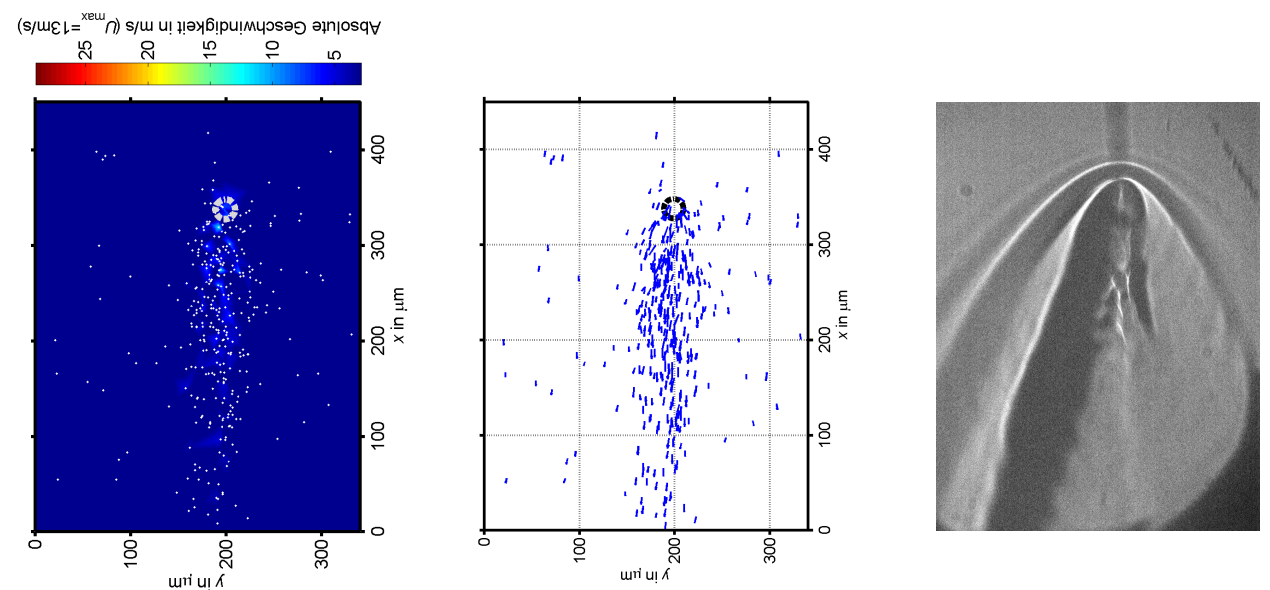

(b) $D=20 \mu \mathrm{m}, U_{0}=29 \mathrm{~m} \mathrm{~s}^{-1}$ und $h=80 \mu \mathrm{m}$

Abbildung F.10: Geschwindigkeit am Substrat für konstante Tropfengröße $D$ und Tropfengeschwindigkeit $U_{0}$ in Abhängigkeit von der Dicke des Flüssigkeitsfilms $h$. Geschwindigkeitsverteilung (linkes Bild), Pfeildarstellung der Strömungsrichtung und -geschwindigkeit (mittleres Bild) und Aufnahme im Durchlicht (rechtes Bild). Weiße Punkte im linken Bild entsprechen den Messpunkten/Pfeilen im mittleren Bild. Ein gestrichelter Kreis zeigt die Tropfengröße und Einschlagsposition. 

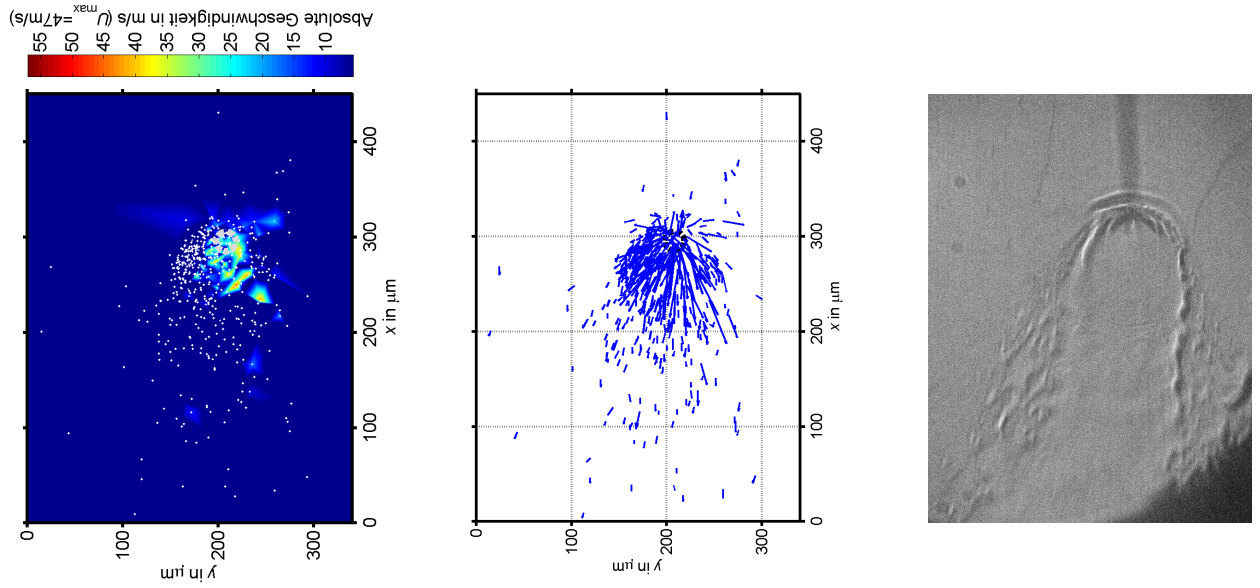

(a) $D=20 \mu \mathrm{m}, U_{0}=57 \mathrm{~m} \mathrm{~s}^{-1}$ und $h=0 \mu \mathrm{m}$
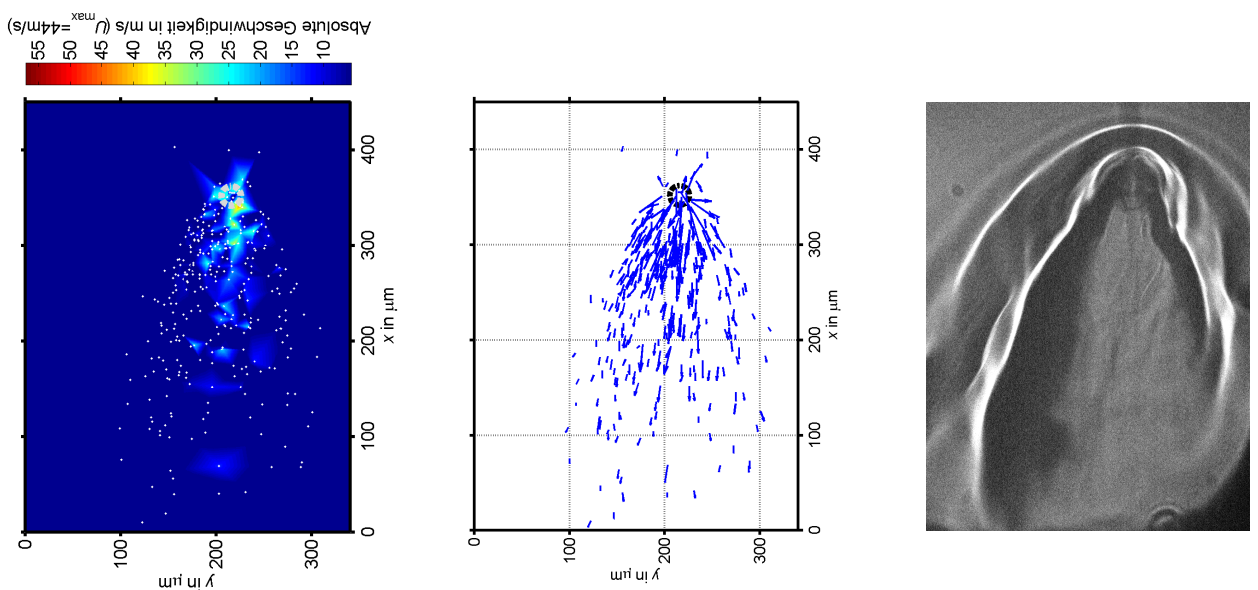

(b) $D=20 \mu \mathrm{m}, U_{0}=57 \mathrm{~m} \mathrm{~s}^{-1}$ und $h=80 \mu \mathrm{m}$

Abbildung F.11: Geschwindigkeit am Substrat für konstante Tropfengröße $D$ und Tropfengeschwindigkeit $U_{0}$ in Abhängigkeit von der Dicke des Flüssigkeitsfilms $h$. Geschwindigkeitsverteilung (linkes Bild), Pfeildarstellung der Strömungsrichtung und -geschwindigkeit (mittleres Bild) und Aufnahme im Durchlicht (rechtes Bild). Weiße Punkte im linken Bild entsprechen den Messpunkten/Pfeilen im mittleren Bild. Ein gestrichelter Kreis zeigt die Tropfengröße und Einschlagsposition. 

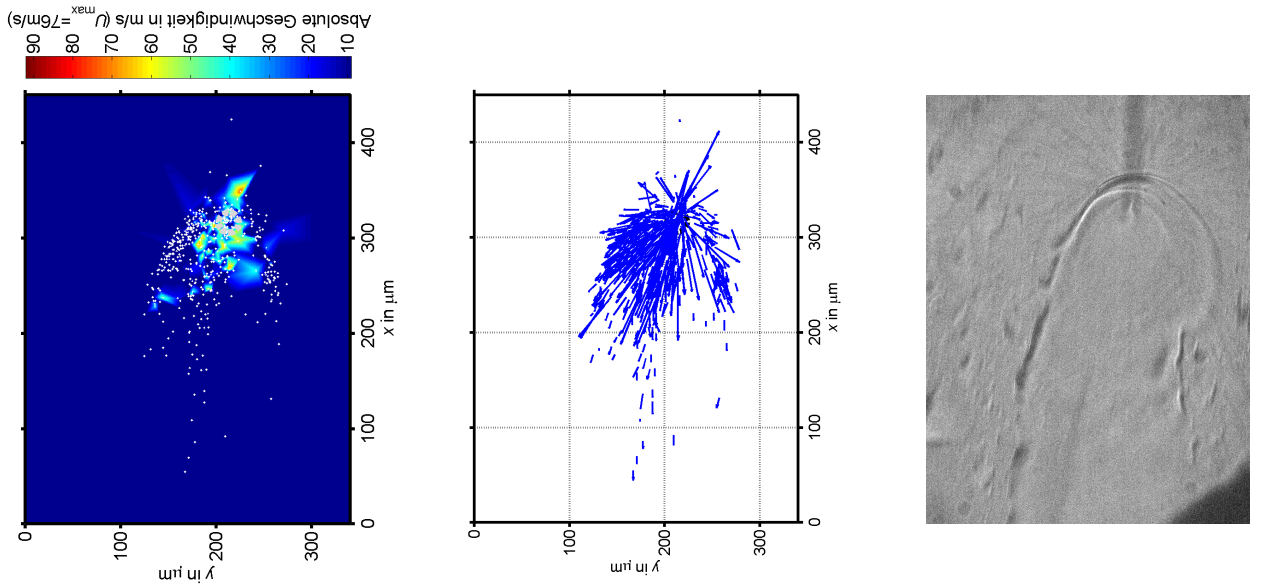

(a) $D=21 \mu \mathrm{m}, U_{0}=92 \mathrm{~m} \mathrm{~s}^{-1}$ und $h=0 \mu \mathrm{m}$
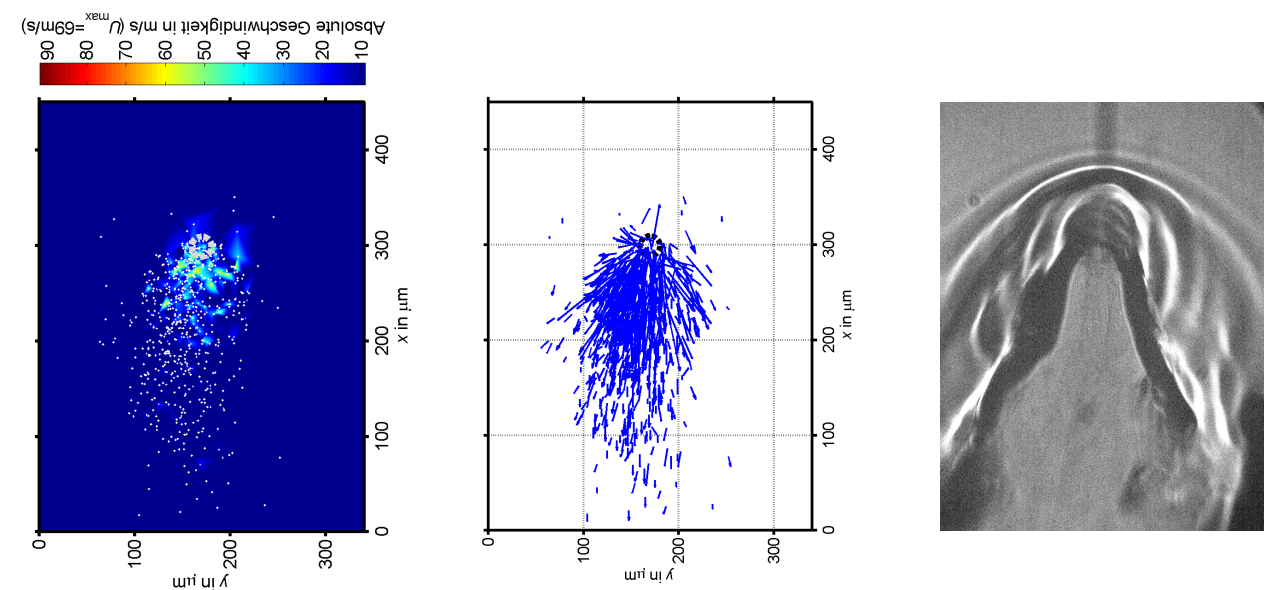

(b) $D=21 \mu \mathrm{m}, U_{0}=92 \mathrm{~m} \mathrm{~s}^{-1}$ und $h=80 \mu \mathrm{m}$

Abbildung F.12: Geschwindigkeit am Substrat für konstante Tropfengröße $D$ und Tropfengeschwindigkeit $U_{0}$ in Abhängigkeit von der Dicke des Flüssigkeitsfilms $h$. Geschwindigkeitsverteilung (linkes Bild), Pfeildarstellung der Strömungsrichtung und -geschwindigkeit (mittleres Bild) und Aufnahme im Durchlicht (rechtes Bild). Weiße Punkte im linken Bild entsprechen den Messpunkten/Pfeilen im mittleren Bild. Ein gestrichelter Kreis zeigt die Tropfengröße und Einschlagsposition. 



\section{Symbolverzeichnis}

\section{Symbole}

\begin{tabular}{|c|c|c|}
\hline Variable & Bedeutung & Einheit \\
\hline$A$ & Fläche & $\mathrm{m}^{2}$ \\
\hline$a$ & Radius & $\mathrm{m}$ \\
\hline$C$ & freier Parameter & - \\
\hline$C_{\mathrm{D}}$ & Strömungswiderstandskoeffizient & - \\
\hline$c$ & Schallgeschwindigkeit & $\mathrm{m} \mathrm{s}^{-1}$ \\
\hline$D$ & Durchmesser & $\mathrm{m}$ \\
\hline$E$ & Elektrisches Feld & $\mathrm{Vm}^{-1}$ \\
\hline$E$ & Energie & $\mathrm{J}$ \\
\hline$e$ & Ladung eines Elektrons & $1,602 \times 10^{-19} \mathrm{C}$ \\
\hline$F$ & Kraft & $\mathrm{N}$ \\
\hline$f$ & Frequenz & $\mathrm{Hz}$ \\
\hline$g$ & Erdbeschleunigung & $9,81 \mathrm{~m} \mathrm{~s}^{-2}$ \\
\hline$k$ & Wellenzahl & $\mathrm{m}^{-1}$ \\
\hline$L$ & Länge & $\mathrm{m}$ \\
\hline$M$ & Vergrößerung & - \\
\hline$m$ & Masse & $\mathrm{kg}$ \\
\hline$N A$ & numerische Apertur & - \\
\hline$n$ & Brechungsindex & - \\
\hline$n$ & Zählvariable & - \\
\hline$O h$ & Ohnesorge-Zahl (Definition: $\mu / \sqrt{\rho \sigma d}$ ) & - \\
\hline$p$ & Druck & $\mathrm{Pa}$ \\
\hline$Q$ & Ladung & $\mathrm{C}$ \\
\hline$Q$ & Volumenstrom & $\mathrm{m}^{3} \mathrm{~s}^{-1}$ \\
\hline$R, r$ & Radius & $\mathrm{m}$ \\
\hline$R e$ & Reynolds-Zahl (Definition: $\rho U d / \mu$ ) & - \\
\hline
\end{tabular}




$\begin{array}{lll}t & \text { Zeit } & \mathrm{S} \\ U, u & \text { Geschwindigkeit } & \mathrm{m} \mathrm{s}^{-1} \\ U & \text { Spannung } & \mathrm{V} \\ W e & \left.\text { Weber-Zahl (Definition: } \rho U^{2} d / \sigma\right) & - \\ x & \text { x-Koordinate } & \mathrm{m} \\ y & \text { y-Koordinate } & \mathrm{m} \\ z & \text { z-Koordinate } & \mathrm{m} \\ \alpha & \text { Winkel } & \circ \\ \varepsilon_{0} & \text { Permittivität des Vakuums } & 8,845 \times 10^{-12} \mathrm{~A} \mathrm{~s} \mathrm{~V}^{-1} \mathrm{~m} \\ \varepsilon_{\mathrm{r}} & \text { relative Permittivität } & - \\ \lambda & \text { Wellenlänge } & \mathrm{m} \\ \mu & \text { dynamische Viskosität } & \mathrm{Pa} \mathrm{s}^{-1} \\ \nu & \text { kinematische Viskosität } & \mathrm{m}^{2} \mathrm{~s}^{-1} \\ \theta & \text { Azimutalwinkel } & \circ \\ \rho & \text { Dichte } & \mathrm{kg} \mathrm{m}^{-3} \\ \sigma & \text { Oberflächenspannung } & \mathrm{N} \mathrm{m}^{-1} \\ \sigma_{\mathrm{R}} & \text { spezifischer Leitwert } & \mathrm{S} \mathrm{m}^{-1} \\ \tau & \text { Zeit } & \mathrm{S} \\ \omega & \text { Kreisfrequenz } & \mathrm{rad} \mathrm{s}^{-1}\end{array}$




\section{Literaturverzeichnis}

[1] A. M. Worthington: A study of splashes. Longmans, Green, New York, 1908.

[2] M. ReIN: Phenomena of liquid drop impact on solid and liquid surfaces. Fluid Dyn. Res. 12(2), 61, 1993.

[3] M. REIN: Drop-surface Interactions. Nummer 456 in CISM International Centre for Mechanical Sciences. Springer Vienna, 2002.

[4] R. Rioboo, M. Marengo und C. Tropea: Time evolution of liquid drop impact onto solid, dry surfaces. Exp. Fluids 33(1), 112-124, 2002.

[5] I. V. Roisman, R. Rioboo und C. Tropea: Normal impact of a liquid drop on a dry surface: model for spreading and receding. Proc. Royal Soc. London A 458(2022), 1411-1430, 2002.

[6] C. Clanet, C. Béguin, D. Richard und D. Quére: Maximal deformation of an impacting drop. J. Fluid Mech. 517, 199-208, 2004.

[7] A. YARIN: Drop impact dynamics: splashing, spreading, receding, bouncing... Annu. Rev. Fluid Mech. 38, 159-192, 2006.

[8] I. V. Roisman, E. Berberovic und C. Tropea: Inertia dominated drop collisions. I. On the universal flow in the lamella. Phys. Fluids 21, 052103-1 - 052103-10, 2009.

[9] J. Eggers, M. A. Fontelos, C. Josserand und S. Zaleski: Drop dynamics after impact on a solid wall: Theory and simulations. Phys. Fluids 22(6), 062101-1-062101-13, 2010.

[10] S. T. Thoroddsen und J. SAKakibara: Evolution of the fingering pattern of an impacting drop. Phys. Fluids 10(6), 1359-1374, 1998.

[11] N. Z. Mehdizadeh, S. Chandra und J. Mostahnimi: Formation of fingers around the edges of a drop hitting a metal plate with high velocity. J. Fluid Mech. 510, 353-373, 2004. 
[12] M. Rein und J.-P. Delplanque: The role of air entrainment on the outcome of drop impact on a solid surface. Act. Mech. 201(1-4), 105-118, 2008.

[13] A. L. YARIN und D. A. WeISS: Impact of drops on solid surfaces: selfsimilar capillary waves, and splashing as a new type of kinematic discontinuity. J. Fluid Mech. 283, 141-173, 1995.

[14] L. Xu, W. W. Zhang und S. R. NAGel: Drop splashing on a dry smooth surface. Phys. Rev. Lett. 94, 184505, 2005.

[15] S. Mandre, M. Mani und M. P. Brenner: Precursors to Splashing of Liquid Droplets on a Solid Surface. Phys. Rev. Lett. 102, 134502, 2009.

[16] M. Mani, S. Mandre und M. P. Brenner: Events before droplet splashing on a solid surface. J. Fluid Mech. 647, 163-185, 2010.

[17] K.-L. PAn, K.-C. Tseng und C.-H. WAng: Breakup of a droplet at high velocity impacting a solid surface. Exp. Fluids 48(1), 143-156, 2010.

[18] S. T. Thoroddsen, M.-J. Thoraval, K. Takehara und T. G. Etoh: Droplet splashing by a slingshot mechanism. Phys. Rev. Lett. 106, 034501, 2011.

[19] S. MANDRE und M. P. BREnNER: The mechanism of a splash on a dry solid surface. J. Fluid Mech. 690, 148-172, 2012.

[20] J. M. Kolinski, L. Mahadevan und S. M. Rubinstein: Drops can bounce from perfectly hydrophilic surfaces. Europhys. Lett. 108(2), 24001, 2014 .

[21] D. A. WeIsS und A. L. YARIN: Single drop impact onto liquid films: neck distortion, jetting, tiny bubble entrainment, and crown formation. J. Fluid Mech. 385, 229-254, 1999.

[22] K. K. Haller, Y. Ventikos und D. Poulikakos: Computational study of high-speed liquid droplet impact. J. Appl. Phys. 92(5), 2821-2828, 2002.

[23] A. Mongruel, V. Daru, F. Feuillebois und S. Tabakova: Early postimpact time dynamics of viscous drops onto a solid dry surface. Phys. Fluids 21(3), 032101-1-032101-13, 2009.

[24] G. Cossali, A. Coghe und M. Marengo: The impact of a single drop on a wetted solid surface. Exp. Fluids 22(6), 463-472, 1997. 
[25] E. Berberović, N. P. van Hinsberg, S. Jakirlić, I. V. Roisman und C. Tropea: Drop impact onto a liquid layer of finite thickness: Dynamics of the cavity evolution. Phys. Rev. E 79, 036306, 2009.

[26] N. P. van Hinsberg, M. Budakli, S. Göhler, E. Berberović, I. V. Roisman, T. Gambaryan-Roisman, C. Tropea und P. Stephan: Dynamics of the cavity and the surface film for impingements of single drops on liquid films of various thicknesses. J. Colloid Interface Sci. 350(1), 336 $-343,2010$.

[27] M. M. DRISCOLL und S. R. NAGEL: Ultrafast interference imaging of air in splashing dynamics. Phys. Rev. Lett. 107, 154502, 2011.

[28] W. Bouwhuis, R. C. A. van der Veen, T. Tran, D. L. Keij, K. G. Winkels, I. R. Peters, D. van Der Meer, C. Sun, J. H. SnoeiJer und D. LOHSE: Maximal air bubble entrainment at liquid-drop impact. Phys. Rev. Lett. 109, 264501, 2012.

[29] J. M. Kolinski, S. M. Rubinstein, S. Mandre, M. P. Brenner, D. A. Weitz und L. MAHADEvan: Skating on a film of air: drops impacting on a surface. Phys. Rev. Lett. 108, 074503, 2012.

[30] J. De Ruiter, J. M. Oh, D. van den Ende und F. Mugele: Dynamics of collapse of air films in drop impact. Phys. Rev. Lett. 108, 074505, 2012.

[31] R. C. A. van der Veen, T. Tran, D. Lohse und C. Sun: Direct measurements of air layer profiles under impacting droplets using high-speed color interferometry. Phys. Rev. E 85, 026315-1 - 026315-6, 2012.

[32] J. EGGERs: Nonlinear dynamics and breakup of free-surface flows. Rev. Mod. Phys. 69(3), 865, 1997.

[33] E. R. LEE: Microdrop generation. CRC Press, Boca Raton, 2002.

[34] M. MCCARThy und N. Molloy: Review of stability of liquid jets and the influence of nozzle design. Chem. Eng. J. 7(1), 1-20, 1974.

[35] S. P. Lin und R. D. REITZ: Drop and spray formation from a liquid jet. Annu. Rev. Fluid Mech. 30, 85-105, 1998.

[36] O. A. BASARAN: Small-scale free surface flows with breakup: Drop formation and emerging applications. AIChE J. 48(9), 1842-1848, 2002.

[37] J. Eggers und E. Villermaux: Physics of liquid jets. Rep. Prog. Phys. 71(3), 036601, 2008. 
[38] J. W. S. Rayleigh: On the instability of jets. Proc. Lond. Math. Soc 10, 4-13, 1878.

[39] J. A. F. Plateau: Statique Expérimentale et Théorique des Liquides Soumis aux Seules Forces Moléculaire, Band 2. Gauthiers-Villars, Paris, 1873.

[40] N. LindBlad und J. SchneIDER: Production of uniform-sized liquid droplets. J. Sci. Instrum 42(8), 635-638, 1965.

[41] E. K. Dabora: Production of monodisperse sprays. Rev. Sci. Instrum. 38(4), 502-506, 1967.

[42] W. Streule, T. Lindemann, G. Birkle, R. Zengerle und P. KolTAY: PipeJet: a simple disposable dispenser for the nano- and microliter range. J. Assoc. Lab. Autom. 9(5), 300-306, 2004.

[43] T. Driessen, R. Jeurissen, H. Wijshoff, F. Toschi und D. Lohse: Stability of viscous long liquid filaments. Phys. Fluids 25(6), 062109, 2013.

[44] S. D. Hoath, S. Jung und I. M. Hutchings: A simple criterion for filament break-up in drop-on-demand inkjet printing. Phys. Fluids 25(2), 021701, 2013.

[45] M. ORME: A novel technique of rapid solidification net-form materials synthesis. J. Mater. Eng. Perform. 2(3), 399-405, 1993.

[46] R. N. Berglund und B. Y. H. Liu: Generation of monodisperse aerosol standards. Environ. Sci. Technol. 7(2), 147-153, 1973.

[47] G. Brenn, F. Durst und C. Tropea: Monodisperse sprays for various purposes - their production and characteristics. Part. Part. Syst. Charact. 13(3), 179-185, 1996.

[48] A. Taberner, N. C. Hogan und I. W. Hunter: Needle-free jet injection using real-time controlled linear lorentz-force actuators. Med. Eng. Phys. 34(9), 1228 - 1235, 2012.

[49] A. HAenLein: Über den Zerfall eines Flüssigkeitsstrahles. Forsch. Geb. Ing. A 2, 139-149, 1931.

[50] S. W. PARK und C. S. LEE: Macroscopic and microscopic characteristics of a fuel spray impinged on the wall. Exp. Fluids 37(5), 745-762, 2004.

[51] A. Moreira, A. Moita und M. PanaO: Advances and challenges in explaining fuel spray impingement: How much of single droplet impact research is useful? Prog. Energ. Combust. 36(5), 554 - 580, 2010. 
[52] S. V. Klinkov, V. F. Kosarev und M. Rein: Cold spray deposition: significance of particle impact phenomena. Aerosp. Sci. Technol. 9(7), 582 - 591, 2005.

[53] A. McDonald, M. Lamontagne, C. Moreau und S. Chandra: Impact of plasma-sprayed metal particles on hot and cold glass surfaces. Thin Solid Films 514(1-2), 212 - 222, 2006.

[54] M. Watanabe, T. Sanada, A. Hayashida und Y. Isago: Cleaning technique using high-speed steam-water mixed spray. Solid State Phenom. 145146, 43-46, 2009.

[55] T. Tanaka, M. Sato, M. Kobayashi und H. Shirakawa: Development of a novel advanced spray technology based on investigation of droplet energy and pattern damage. Solid State Phenom. 187, 153-156, 2012.

[56] H. F. Okorn-Schmidt, F. Holsteyns, A. Lippert, D. Mui, M. KaWaguchi, C. Lechner, P. E. Frommhold, T. Nowak, F. Reuter, M. B. PiquÉ und OTHERS: Particle cleaning technologies to meet advanced semiconductor device process requirements. ECS J. Solid State Sci. Technol. 3(1), N3069-N3080, 2014.

[57] M. T. Andreas, K. Wostyn, M. Wada, T. Janssens, K. Kenis, T. BEARDA und P. W. MERTENS: High velocity aerosol cleaning with organic solvents: particle removal and substrate damage. Solid State Phenom. 145-146, 39-42, 2009.

[58] K. Xu, S. Pichler, K. Wostyn, G. Cado, C. Springer, G. W. GaLe, M. Dalmer, P. W. Mertens, T. Bearda, E. Gaulhofer und D. PODLESNIK: Removal of nano-particles by aerosol spray: effect of droplet size and velocity on cleaning performance. Solid State Phenom. 145, 31-34, 2009.

[59] D. Ahn, J. HA und D. Kim: Development of an opto-hydrodynamic process to remove nanoparticles from solid surfaces. Appl. Surf. Sci. 265(0), $630-$ 636, 2013.

[60] C. Kennedy und J. FiELD: Damage threshold velocities for liquid impact. J. Mater. Sci. 35(21), 5331-5339, 2000.

[61] Y. Hirota, I. Kanno, K. Fujiwara, H. Nagayasu und S. Shimose: Damage-free wafer cleaning by water and gas mixture jet. In: Semiconductor Manufacturing, 2005. ISSM 2005, IEEE International Symposium on, Seiten 219-222, 2005. 
[62] T. Sanada, M. Watanabe, M. Shirota, M. Yamase und T. Saito: Impact of high-speed steam-droplet spray on solid surface. Fluid Dyn. Res. 40(7), 627-636, 2008.

[63] M. Sato, K. Sotoku, K. Yamaguchi, T. Tanaka, M. Kobayashi und S. NADAhara: Analysis on Threshold Energy of Particle Removal in Spray Cleaning Technology. ECS Transactions 41(5), 75-82, 2011.

[64] F. TARDiF, A. DANEL und O. RACCURT: Understanding of wet and alternative particle removal processes in microelectronics: theoretical capabilities and limitations. J. Telecommun. Inf. Technol. Seiten 11-19, 2005.

[65] P. W. Mertens, G. Vereecke, R. Vos, S. Arnauts, F. Barbagini, T. Bearda, S. DeGendt, C. Demaco, A. Eitoku, M. Frank, W. Fyen, L. Hall, D. Hellin, F. Holsteyns, E. Kesters, M. Claes, K. Kim, K. Kenis, H. Kraus, R. Hoyer, T. Q. Le, M. Lux, K.-T. LeE, M. Kocsis, T. Kotani, S. Malhouitre, A. Muscat, B. Onsia, S. GaRaud, J. Rip, K. Sano, S. Sioncke, J. Snow, J. Van Hoeymissen, K. Wostyn, K. Xu, V. Parachiev und M. Heyns: Roadblocks and Critical Aspects of Cleaning for Sub-65nm Technologies. In: VLSI Technology, Systems, and Applications, 2006 International Symposium on, Seiten 1-4, 2006.

[66] S. Q. Armster, J.-P. Delplanque, M. Rein und E. J. Lavernia: Thermo-fluid mechanisms controlling droplet based materials processes. Int. Mater. Rev. 47(6), 265-301, 2002.

[67] R. MetTin: Application for the establishment and funding of the Christian Doppler Laboratory for Cavitation and Micro Erosion, 2008.

[68] Y. H. C. Chien, M. Yeh, S. Ku, C. Yang, C. Chen, S. Jang, K. Izumoto, K. Sotoku, T. TAnaka, M. Sato, H. Shirakawa und M. TAnaKA: Physical cleaning enhancement using advanced spray with uniform droplet control. In: Solid State Phenom, Band 195, Seiten 195-197. Trans Tech Publ, 2013.

[69] I. Kanno, N. YokoI und K. SATO: Wafer cleaning by water and gas mixture with high velocity. Electrochem. Soc. Proc. 98, 54-61, 1997.

[70] C. W. VISSER: Fundamentals and applications of fast micro-drop impact. Dissertation, Universität Twente, 2014.

[71] J. P. DEAR und J. E. FIELD: High-speed photography of surface geometry effects in liquid/solid impact. J. Appl. Phys. 63(4), 1015-1021, 1988. 
[72] J. E. Field, J. P. DeAR und J. E. OGREN: The effects of target compliance on liquid drop impact. J. Appl. Phy. 65(2), 533-540, 1989.

[73] R. J. HAnd, J. E. FiEld und D. Townsend: The use of liquid jets to simulate angled drop impact. J. Appl. Phys 70(11), 7111-7118, 1991.

[74] M. REIN: Wave phenomena during droplet impact. In: IUTAM Symposium on Waves in Liquid/Gas and Liquid/Vapour Two-Phase Systems, Seiten 171-190. Springer, 1995.

[75] M. REIN: Nonlinear analysis of two-dimensional compressible liquid-liquid impact. Eur. J. Mech. B-Fluid 14(3), 301-322, 1995.

[76] F. Holsteyns: Removal of nanoparticulate contaminants from semiconductor substrates by megasonic cleaning. Dissertation, Katholieke Universiteit Leuven, 2008.

[77] C. W. Visser, M. V. Gielen, Z. Hao, S. Le Gac, D. Lohse und C. Sun: Quantifying cell adhesion through impingement of a controlled microjet. Biophys. J. 108(1), 23-31, 2015.

[78] P. E. Frommhold, R. Mettin and C.-D. Ohl: Height-resolved velocity measurement of the boundary flow during liquid impact on dry and wetted solid substrates. Exp. Fluids 56(4), 1-17, 2015.

[79] G. BRENN: On the controlled production of sprays with discrete polydisperse drop size spectra. Chem. Eng. Sci. 55(22), 5437-5444, 2000.

[80] C. WeBER: Zum Zerfall eines Flüssigkeitsstrahles. ZAMM - Z. Angew. Math. Mech. 11(2), 136-154, 1931.

[81] P. E. Frommhold, A. Lippert, F. L. Holsteyns and R. Mettin: High-speed monodisperse droplet generation by ultrasonically controlled micro-jet breakup. Exp. Fluids 55(4), 1-12, 2014.

[82] J. M. Schneider, N. R. Lindblad, C. D. Hendricks and J. M. CrowLEY: Stability of an Electrified Liquid Jet. J. Appl. Phys. 38(6), 25992605, 1967.

[83] P. Walzel: Koaleszenz von Flüssigkeitsstrahlen an Brausen. Chem. Ing. Tech. 52(8), 652-654, 1980.

[84] W. von Ohnesorge: Die Bildung von Tropfen an Düsen und die Auflösung flüssiger Strahlen. Z. Angew. Math. Mech. 16(6), 355-358, 1936.

[85] C. Dumouchel: On the experimental investigation on primary atomization of liquid streams. Exp. Fluids 45(3), 371-422, 2008. 
[86] R. P. Grant and S. Middleman: Newtonian jet stability. AIChE J. 12(4), 669-678, 1966.

[87] S. W. J. Smith and H. Moss: Experiments with mercury jets. Proc. R. Soc. Lond. A 93(652), 373-393, 1917.

[88] A. A. Castrejón-Pita, J. R. Castrejón-Pita and I. M. Hutchings: Breakup of liquid filaments. Phys. Rev. Lett. 108, 074506, 2012.

[89] A. Prosperetti: Free oscillations of drops and bubbles: the initial-value problem. J. Fluid Mech. 100, 333-347, 1980.

[90] U. Weierstall, R. Doak, J. Spence, D. Starodub, D. Shapiro, P. Kennedy, J. Warner, G. Hembree, P. Fromme and H. Chapman: Droplet streams for serial crystallography of proteins. Exp. Fluids 44(5), 675-689, 2008.

[91] H.-Y. KIM, S.-Y. PARK and K. MiN: Imaging the high-speed impact of microdrop on solid surface. Rev. Sci. Instrum. 74(11), 4930-4937, 2003.

[92] M. Smith and V. Bertola: Particle velocimetry inside Newtonian and non-Newtonian droplets impacting a hydrophobic surface. Exp. Fluids 50(5), 1385-1391, 2011.

[93] N. ERKAN and K. OKAMOTO: Full-field spreading velocity measurement inside droplets impinging on a dry solid surface. Exp. Fluids 55(11), 1-9, 2014 .

[94] M. Versluis: High-speed imaging in fluids. Exp. Fluids 54(2), 1-35, 2013.

[95] C. W. Visser, P. E. Frommhold, S. Wildeman, R. Mettin, D. LoHSE and C. Sun: Dynamics of high-speed micro-drop impact: numerical simulations and experiments at frame-to-frame times below $100 \mathrm{~ns}$. Soft Matter 11, 1708-1722, 2015.

[96] P. E. Frommhold, C. W. Visser, A. Lippert and R. Mettin: Generation of precisely controlled high-speed micro droplets. Konferenzbeitrag, Flow14, Twente, Niederlande, 18.-21. Mai 2014.

[97] C. W. Visser, P. E. Frommhold, R. Mettin, C. Sun and D. Lohse: Microdroplet impact. Konferenzbeitrag, Focus on Microscopy, Sydney, Australien, 13.-16. April 2014.

[98] C.-D. Ohl, X. Huang, C. U. Chan, P. E. Frommhold and A. LipPERT: Water Entry by a Train of Droplets. Konferenzbeitrag, American Physical Society, San Francisco, Kalifornien, 23.-25 November 2014. 
[99] A. Bos, A. Zijlstra, E. Gelderblom and M. Versluis: iLIF: illumination by Laser-Induced Fluorescence for single flash imaging on a nanoseconds timescale. Exp. Fluids 51(5), 1283-1289, 2011.

[100] P. Kim, M. J. Kreder, J. Alvarenga and J. Aizenberg: Hierarchical or not? Effect of the length scale and hierarchy of the surface roughness on omniphobicity of lubricant-infused substrates. Nano Lett. 13(4), 17931799, 2013.

[101] L. RAYLEIGH: On the equilibrium of liquid conducting masses charged with electricity. Philos. Mag. J. Sci. 14(87), 184-186, 1882.

[102] J. M. SCHNEIDER and C. D. HendRICKs: Source of uniform sized liquid droplets. Rev. Sci. Instrum. 35(10), 1349-1350, 1964.

[103] J. Schneider, N. Lindblad and C. Hendricks: An apparatus to study the collision and coalescence of liquid aerosols. J. Colloid Sci. 20(6), $610-$ 616, 1965.

[104] G. Reischl, W. John and W. Devor: Uniform electrical charging of monodisperse aerosols. J. Aerosol Sci. 8(1), 55 - 65, 1977.

[105] A. Atten and S. Oliveri: Charging of drops formed by circular jet breakup. J. Electrostat. 29(1), 73-91, 1992.

[106] M. Orme, J. Courter, Q. Liu, C. Huang and R. Smith: Electrostatic charging and deflection of nonconventional droplet streams formed from capillary stream breakup. Physics of Fluids 12(9), 2224-2235, 2000.

[107] K. Mitsotakis, W. Schneider and E. Zauner: Second-order boundary-layer theory of laminar jet flows. Acta Mech. 53(1-2), 115123, 1984.

[108] J. A. Mulholland, R. K. Srivastava and J. O. L. Wendt: Influence of droplet spacing on drag coefficient in nonevaporating, monodisperse streams. AIAA J. 26(10), 1231-1237, 1988.

[109] H. LEE: Boundary layer around a liquid jet. IBM J. Res. Dev. 21(1), 48-51, 1977.

[110] S. PopInET: An accurate adaptive solver for surface-tension-driven interfacial flows. J. Comput. Phys. 228(16), 5838 - 5866, 2009.

[111] L. Cheng: Dynamic spreading of drops impacting onto a solid surface. Ind. Eng. Chem. Proc. D. D. 16(2), 192-197, 1977. 
[112] D. B. VAN DAM and C. L. CleRC: Experimental study of the impact of an ink-jet printed droplet on a solid substrate. Phys. Fluids 16(9), 3403-3414, 2004 .

[113] C. W. Visser, Y. TAgawa, C. Sun and D. Lohse: Microdroplet impact at very high velocity. Soft Matter 8, 10732-10737, 2012.

[114] S. Chandra and C. T. Avedisian: On the collision of a droplet with a solid surface. Proc. R. Soc. London, Ser. A 432(1884), 13-41, 1991.

[115] M. Pasandideh-Fard, Y. M. QiaO, S. Chandra and J. Mostaghimi: Capillary effects during droplet impact on a solid surface. Phys. Fluids 8(3), 650-659, 1996.

[116] C. Willert, B. Stasicki, J. Klinner and S. Moessner: Pulsed operation of high-power light emitting diodes for imaging flow velocimetry. Meas. Sci. Technol. 21(7), 075402, 2010.

[117] N. A. Buchmann, C. E. Willert and J. Soria: Pulsed, high-power LED illumination for tomographic particle image velocimetry. Exp. Fluids 53(5), 1545-1560, 2012.

[118] C. D. Meinhart, S. T. Wereley and J. G. Santiago: PIV measurements of a microchannel flow. Exp. Fluids 27(5), 414-419, 1999.

[119] J. Burns, C. RAmshaw and R. JACHUCK: Measurement of liquid film thickness and the determination of spin-up radius on a rotating disc using an electrical resistance technique. Chem. Eng. Sci. 58(11), 2245 - 2253, 2003.

[120] F. Staudegger, M. W. Hofbaur and H.-J. Kruwinus: Analyses and modeling of a wet-chemical-etch process on rotating silicon wafers with an impinging etchant jet. J. Electrochem. Soc. 156(5), H340-H345, 2009.

[121] T.-S. KIM and M.-U. KIM: The flow and hydrodynamic stability of a liquid film on a rotating disc. Fluid Dyn. Res. 41(3), 035504, 2009.

[122] R. M. WoOD and B. E. WATTS: The flow, heat and mass transfer characteristics of liquid films on rotating discs. Trans. Inst. Chem. Eng. 51, $315-322,1973$.

[123] C. U. Chan and C.-D. OHL: Total-Internal-Reflection-Fluorescence microscopy for the study of nanobubble dynamics. Phys. Rev. Lett. 109, 174501, 2012.

[124] L. D. Landau and E. M. Lifshitz: Fluid Mechanics (Second Edition). Butterworth-Heinemann, Boca Raton, 1987. 




\section{Danksagung}

Ich möchte mich bedanken bei...

... Herrn Prof. Dr. Christoph F. Schmidt für die Ermöglichung dieser Arbeit.

... Herrn Prof. Dr. Stefan Luther für die Übernahme des Korreferats.

... Dr. Robert Mettin für die Betreuung und Durchsicht der Arbeit und für seine kontinuierliche Unterstützung und Anleitung während der Promotion.

... allen Mitgliedern des Christian Doppler Labors für Kavitation und Mikroerosion für die angenehme und motivierende Zusammenarbeit, sowohl im Fachlichen wie auch im Privaten. Nur durch eine funktionierende Arbeitsgruppe ist eine schnelle und lösungsorientierte Erarbeitung wissenschaftlicher Fragestellungen erst möglich. Insbesondere gilt mein Dank Andrea Thiemann, Till Nowak und Fabian Reuter.

... Dr. Frank Holsteyns und Alexander Lippert repräsentativ für die gute Zusammenarbeit mit dem Industriepartner Lam Research AG. Ihre vielfältige Unterstützung und Nähe zu anwendungsbezogenen Fragestellungen ermöglichten neue und herausfordernde Überlegungen und Experimente.

... Claus-Dieter Ohl und Chon U Chan für die effektive und vorantreibende Zusammenarbeit auch über tausende Kilometer hinweg.

... Claas Willem Visser für die vorbildhafte und auf hohem wissenschaftlichen Niveau stattgefundene Zusammenarbeit. Verschiedene Herangehensweisen und Aspekte der vorliegenden Untersuchung wurden maßgeblich durch unsere gemeinsamen Erfahrungen geprägt.

... der feinmechanischen Werkstatt und der Elektronikwerkstatt des Dritten Physikalischen Instituts und allen ihren Mitarbeiterinnen und Mitarbeitern. Stets waren sie allen Überlegungen und Vorschlägen gegenüber offen und hilfsbereit. Ihre fachliche, kompetente Arbeitsweise und schnelle Umsetzungsfähigkeit machten manche Experimente erst möglich. Besonderer Dank gilt hierbei den Leitern Dr. Karl Lautscham und Dieter Hille. 
... Thomas Geiling für die (häufig auch sehr kurzfristigen) Hilfen bei Fragen der IT-Infrastruktur.

... den Sekretärinnen Elke Zech, Nicole Rehbein, Ursula Hahn-Wörgötter und Sabine Huhnold für ihre Unterstützung und manchmal auch Nachsichtigkeit bei organisatorischen und formalen Dingen.

... allen meinen Begleitern während des Studiums. Insbesondere danke ich Welm Pätzold und Elias Blumenröther, die mich vom ersten Tag an begleitet haben und wirklich alle Phasen meines Studiums mitgefühlt und durchlebt haben. Nicht nur ein Mal mussten wir uns gegenseitig stützen, wenn es nicht mehr zu gehen schien.

... Herrn Alfred Rudolph für lautstarke Unterstützung in ruhigen Momenten.

... Anne Biermann, Gisela Grohne, Esther Frommhold und Jürgen Frommhold für das Korrekturlesen der Arbeit.

... meinen Eltern Esther Frommhold und Jürgen Frommhold für die Unterstützung während fast eines Jahrzehnts des Studiums und zuvor fast zwei Jahrzehnten meines Lebens. Ebenso in den Dank eingeschlossen ist meine gesamte Familie. Der Platz an dieser Stelle und mein Wortschatz würden wohl nicht ausreichen, daher einfach nur: Danke!

... meiner Freundin Anne Biermann. Kurz vor Beginn meiner Promotion durfte ich sie kennenlernen und die Tatsache, dass sie mit mir auch anstrengende Zeiten zum Ende der Promotion und zum Beginn einer neuen Arbeitsstelle durchlebt hat, lässt mich zuversichtlich und glücklich in die Zukunft blicken!

... der Christian Doppler Forschungsgesellschaft und der Lam Research AG für die Finanzierung dieser Arbeit. 


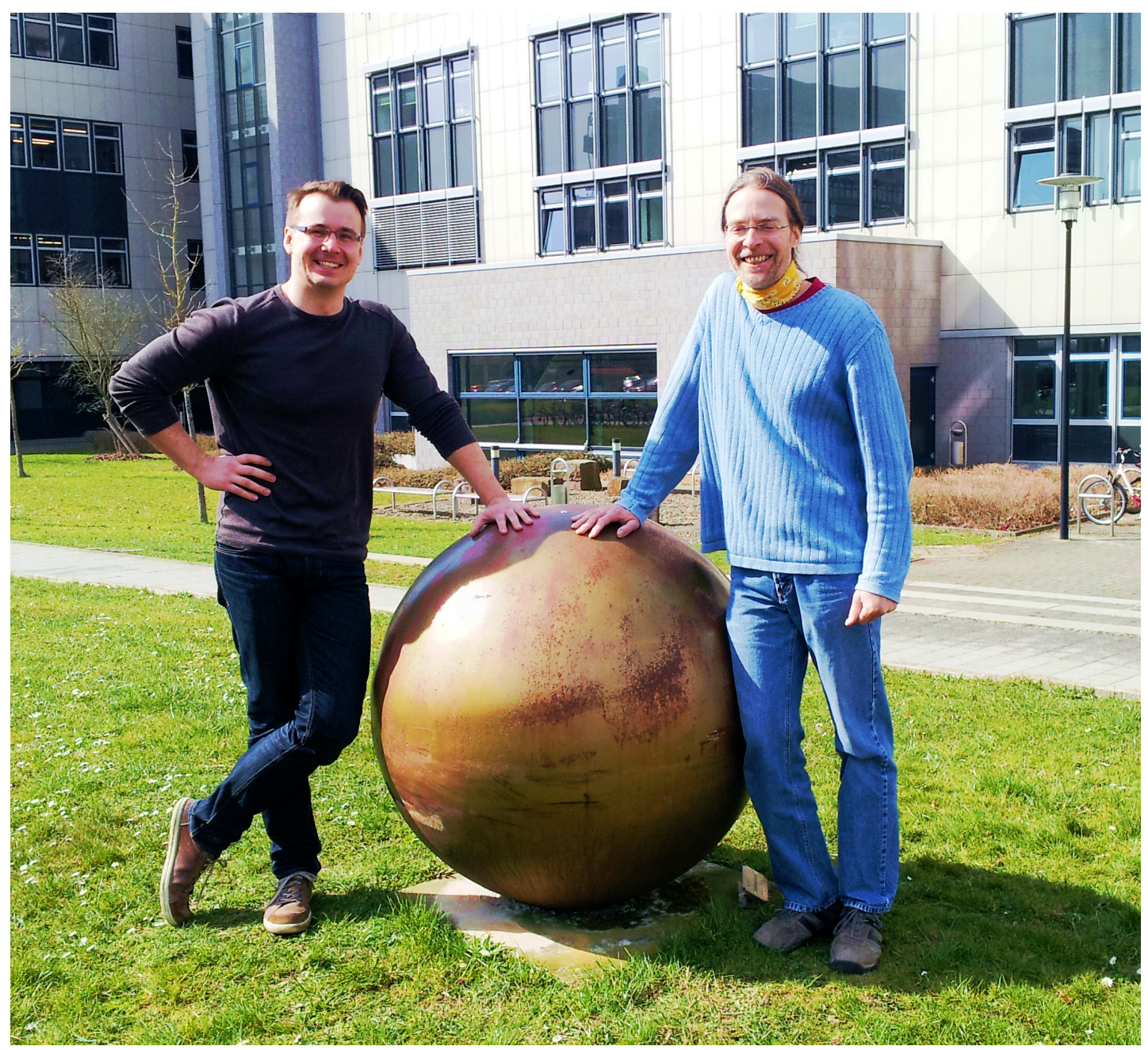

Philipp Erhard Frommhold (links) und Dr. Robert Mettin (rechts). 



\title{
Lebenslauf
}

\author{
Name Philipp Erhard Frommhold \\ Geburtstag 05.07.1985 \\ Geburtsort Wismar \\ Nationalität deutsch
}

1992-1996 Fritz-Reuter-Schule Wismar

1996-2005 Geschwister-Scholl-Gymnasium Wismar

Juni 2005 Abschluss mit der Allgemeinen Hochschulreife

2006-2011 Studiengang Physik an der

Georg-August-Universität Göttingen

September 2009 Erwerb des Hochschulgrades B.Sc. in Physik

September 2011 Erwerb des Hochschulgrades M.Sc. in Physik

Oktober 2011 Beginn der Promotion an der

Georg-August-Universität Göttingen 